Universidad Nacional de La Plata Facultad de Ciencias Exactas

Departamento de Física

\title{
ESTUDIO SOBRE LAS \\ PROPIEDADES ESTRUCTURALES, ELECTRÓNICAS Y MAGNÉTICCAS DE MATERIALES DE INTERÉS TECNOLÓGICO POR MEDIO DE CÁlCULOS CUÁNTICOS
}

Arles Víctor Gil Rebaza

Director $\quad$ : Dr. Eitel L. Peltzer y Blancá

Co-director : Dra. Judith Desimoni

La Plata, 2013 


$$
\text { . }
$$




\section{Agradecimientos}

De antemano deseo agradecer a este hermoso y acogedor país, República Argentina, y a las instituciones que hicieron posible el desarrollo de la presente tesis, tales como la Agencia Nacional de Promoción Científica y Tecnológica (AGENCIA), al Consejo Nacional de Investigaciones Científicas y Técnicas (CONICET), al Departamento de Física de la Facultad de Ciencias Exactas de la Universidad Nacional de La Plata (UNLP) como también al Instituto de Física de La Plata (IFLP).

Con gran respeto y consideración a mis directores, Dr. Eitel L. Peltzer y Blancá y Dra. Judith Desimoni (que en paz descanse), quienes me brindaron su confianza al darme la oportunidad de desarrollar mis estudios doctorales junto a ellos, quienes han sido dos grandes pilares desde el inicio hasta el final del presente trabajo, estando siempre ahí guiándome tanto en el ámbito académico como personal.

A mis padres, Víctor A. Gil Terrones y Eugenia L. Rebaza Oliva, por otorgarme una buena educación desde un inicio, que es la mejor herencia que he obtenido, por haberme apoyado siempre en todos mis estudios e inquie- 
tudes, y supieron encaminarme por las sendas de la lectura y la ciencia. A mis hermanas Leyla I. y Massiel L., a mi hermano Oswer A. con quienes todos juntos formamos la hermosa y unida Familia Gil Rebaza, que a pesar de encontrarme a varios kilómetros de distancia de ellos, sé que siempre han estado y estarán pendientes de mí.

Con mucha emoción, cariño y amor a la mujer que desde hace unos años forma parte de un gran y hermoso proyecto de vida, como es el de formar la Familia Gil Mudarra, ella es mi querida esposa Dra. Azucena M. Mudarra Navarro, quien siempre ha tenido la suficiente paciencia, comprensión y amor hacia mí durante el desarrollo de mi tesis.

También agradezco a toda las personas del IFLP que de alguna u otra forma me apoyaron en el transcurso de mi tesis, con énfasis al Dr. Leo Errico por su disponibilidad en brindarme siempre su ayuda, como también a mis amistades internacionales del IFLP, la denominada Comunidad Latinoamericana, con quienes he compartido muy gratos momentos. 
Del mismo modo que el aumento constante de la entropía es la ley básica del universo, la ley básica de la vida es estar cada vez mejor estructurado y luchar contra la entropía... Václav Havel (1977) 
Dedicado con mucho respeto y cariño a la Dra. Judith Desimoni

(1956-2012) 


\section{Índice}

Lista de Acrónimos

IX

Lista de Figuras $\quad$ X

$\begin{array}{ll}\text { Lista de Tablas } & \text { XVI }\end{array}$

$\begin{array}{ll}\text { Introducción } & 1\end{array}$

1. Fundamento Teórico 4

1.1. Sistema de Muchos Cuerpos . . . . . . . . . . . . . . 4

1.1.1. Aproximación de Born-Oppenheimer . . . . . . . . . 5

1.2. Teoría de la Funcional Densidad . . . . . . . . . . . 6

1.2.1. Teoremas de Hohenberg-Kohn . . . . . . . . . . 7

1.2.2. Ecuación de Kohn-Sham . . . . . . . . . . . . . . 7

1.2.3. Funcional de Intercambio-Correlación . . . . . . . . . . 9

1.2.4. Funcional de la Densidad de Espín . . . . . . . . . . . 10

1.3. Solución de la Ecuación de Kohn-Sham . . . . . . . . . . . . . 11

1.3.1. El Método APW . . . . . . . . . . . . . . . 13

1.3.2. El Método LAPW . . . . . . . . . . . . . . . . . . . 15 
2.1. Antecedentes Experimentales y Teóricos . . . . . . . . . . . 17

2.2. Metodología . . . . . . . . . . . . . . . 27

2.2.1. Ecuación de Estado de Birch-Murnaghan . . . . . . . . 28

2.2.2. Aproximación de la Supercelda . . . . . . . . . . . . . 28

2.3. Cálculo de las Propiedades Hiperfinas con el Método FP-LAPW 32

3. Sistemas Ternarios $X \mathrm{Fe}_{3} \mathrm{~N}(X$ un metal $3 d$ o $4 d)$

3.1. Compuestos Ternarios $X \mathrm{Fe}_{3} \mathrm{~N}(\mathrm{X}$ metal $3 d) \ldots \ldots$

3.1.1. Sitio de sustitución preferencial del átomo X . . . . . 35

3.1.2. Compuestos Ternarios $\mathrm{XFe}_{3} \mathrm{~N}$ con Estructura $P m \overline{3} m \quad$. 39

3.1.3. Compuestos Ternarios $\mathrm{XFe}_{3} \mathrm{~N}$ con Estructura $P 4 / m m m 54$

3.2. Compuestos Ternarios $X \mathrm{Fe}_{3} \mathrm{~N}(\mathrm{X}$ metal $4 d) \ldots \ldots$

3.2.1. Sitio de Sustitución Preferencial del Átomo X . . . . . . 71

3.2.2. Propiedades Estructurales . . . . . . . . . . . . . 72

3.2.3. Propiedades Magnéticas e Hiperfinas . . . . . . . . 76

3.3. Conclusiones . . . . . . . . . . . . . . . . . . . . 82

4. Estudio de los Compuestos Ternarios $M_{x} \mathrm{Fe}_{4-x} \mathrm{~N}(M=\mathrm{Mn}, \mathrm{Co}, \mathrm{Ni}) 84$

4.1. Compuestos Ternarios $\mathrm{Mn}_{x} \mathrm{Fe}_{4-x} \mathrm{~N} \ldots \ldots \ldots \ldots$

4.1.1. Determinación del Estado Fundamental . . . . . . . . 85

4.1.2. Estructura Electrónica, Propiedades Magnéticas e Hiperfinas $\ldots \ldots \ldots \ldots \ldots \ldots \ldots$ 
4.2. Compuestos Ternarios $\mathrm{Co}_{x} \mathrm{Fe}_{4-x} \mathrm{~N}_{\text {y Ni}} \mathrm{Ni}_{4} \mathrm{Fe}_{4-x} \mathrm{~N} \ldots \ldots \ldots$. . . 97

4.2.1. Propiedades Estructurales . . . . . . . . . . . . . . . . 98

4.2.2. Propiedades Magnéticas e Hiperfinas . . . . . . . . 102

4.3. Conclusiones . . . . . . . . . . . . . . . . . . . . . . . . . . 109

5. Estudio de los Compuestos Ternarios $M_{x} \mathrm{Fe}_{4-x} \mathbf{N}(M=\mathrm{Al}, \mathrm{Ga}$, In) 111

5.1. Estado Fundamental de los Compuestos $M \mathrm{Fe}_{3} \mathrm{~N}(M=\mathrm{Al}, \mathrm{Ga}$,

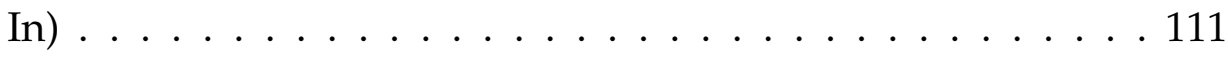

5.2. Compuestos Ternarios $M_{x} \mathrm{Fe}_{4-x} \mathrm{~N}(M=\mathrm{Al}, \mathrm{Ga}, \mathrm{In}) \ldots \ldots \ldots$

5.2.1. Propiedades Estructurales . . . . . . . . . . . . . 118

5.2.2. Estructura Electrónica, Propiedades Magnéticas e Hi-

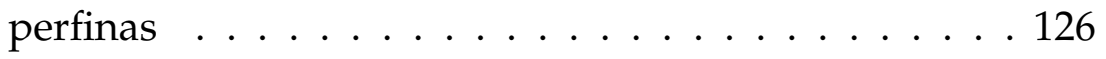

5.3. Conclusiones . . . . . . . . . . . . . . . . . . . . . . . . 144 


\section{Lista de Acrónimos}

$\begin{array}{ll}\text { APW } & \text { Augmented Plane Wave } \\ \text { ASW } & \text { Augmented Spherical Wave } \\ \text { BCC } & \text { Body Center Cube } \\ \text { CVM } & \text { Cluster Variation Method } \\ \text { DC-MS } & \text { Direct Current-Magnetron Sputtering } \\ \text { DFT } & \text { Density Functional Theory } \\ \text { DOS } & \text { Density of States } \\ \text { FP-LAPW } & \text { Full Potential Linearized Augmented Plane Wave } \\ \text { FP-LASTO } & \text { Full Potential Linearized Augmented Slater Type Orbital } \\ \text { FSM } & \text { Fixed Spin Moment } \\ \text { GGA } & \text { Generalized Gradient Approximation } \\ \text { LAPW } & \text { Linearized Augmented Plane Wave } \\ \text { LCAO } & \text { Linear Combination of Atomic Orbitals } \\ \text { LDA } & \text { Local Density Approximation } \\ \text { LMTO } & \text { Linearized Muffin-Tin Orbital } \\ \text { MBE } & \text { Molecular Beam Epitaxy } \\ \text { MS- } X_{\alpha} & \text { Multiple-Scattering } X_{\alpha} \text { Method } \\ \text { PAW-PW } & \text { Projector Augmented Wave - Plane Wave } \\ \text { PBE } & \text { Perdew-Burke-Ernzerhof Funcional } \\ \text { PLD } & \text { Pulsed Laser Deposition } \\ \text { PP-PW } & \text { Pseudo Potentials-Plane Wave } \\ \text { RT } & \text { Room Temperature } \\ \text { USPP-PW } & \text { Ultrasoft Pseudo Potentials-Plane Wave } \\ & \\ & \end{array}$




\section{Lista de Figuras}

1.1. Diagrama de flujo del proceso autoconsistente de la Ecuación de Kohn-Sham. . . . . . . . . . . . . . . . . . . . . 12

1.2. División del espacio de un sólido: Esferas de muffin-tin $S_{M T, \alpha}$ y $S_{M T, \beta}$ centradas en los átomos $\alpha$ y $\beta$ y el espacio intersticial $(I) \ldots \ldots 13$

2.1. Estructura Cristalina del $\gamma^{\prime}-\mathrm{Fe}_{4} \mathrm{~N} \ldots \ldots \ldots \ldots$

2.2. Supercelda $2 \times 2 \times 2$ de $\gamma^{\prime}-\mathrm{Fe}_{4} \mathrm{~N}$ utilizada para obtener diferentes valores de $x$ en los compuestos ternarios $M_{x} \mathrm{Fe}_{4-x} \mathrm{~N}$ para el caso que a) el átomo $M$ sustituye a un $\mathrm{Fe} I, \mathrm{~b}$ ) el átomos $M$ sustituye a un FeII y c) subred cúbica formada por los 8 átomos de FeI o FeII que podrán ser reemplazados por un átomo $M \ldots$. . . . . . . . . . . . 30

2.3. Distintas configuraciones $C_{i \ldots j}^{k}$ correspondientes a las diferentes concentraciones $x \ldots \ldots \ldots \ldots \ldots \ldots \ldots \ldots$. . . . . . . . . . . . . . . . .

3.1. Estructuras cristalinas a) $P m \overline{3} m$ y b) $P 4 / m m m$ de los compuestos

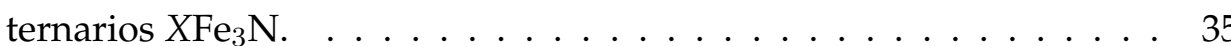

3.2. Curvas volumen - energía para los compuestos ternarios: a) $\mathrm{ScFe}_{3} \mathrm{~N}$, b) $\left.\left.\left.\left.\left.\left.\mathrm{TiFe}_{3} \mathrm{~N}, \mathrm{c}\right) \mathrm{VFe}_{3} \mathrm{~N}, \mathrm{~d}\right) \mathrm{CrFe}_{3} \mathrm{~N}, \mathrm{e}\right) \mathrm{MnFe}_{3} \mathrm{~N}, \mathrm{f}\right) \mathrm{CoFe}_{3} \mathrm{~N}, \mathrm{~g}\right) \mathrm{NiFe}_{3} \mathrm{~N}, \mathrm{~h}\right) \mathrm{CuFe}{ }_{3} \mathrm{~N}$, i) $\mathrm{ZnFe}_{3} \mathrm{~N}$ para las estructuras cristalinas $P m \overline{3} m$ y $P 4 / m m m$. . . . . . 37

3.3. Valor de $\Delta E$ para cada compuesto ternario $X \mathrm{Fe}_{3} \mathrm{~N}$. . . . . . . . . 39

3.4. Variación del parámetro de red de los compuestos $X \mathrm{Fe}_{3} \mathrm{~N}$ en función del número atómico $(Z)$ del átomo X . . . . . . . . . . . . . . . . . 41 
3.5. Variación del parámetro de red (a) de los compuestos ternarios $\mathrm{XFe}_{3} \mathrm{~N}$ respecto al $r_{\text {met }}$ del átomo X. . . . . . . . . . . . . . . . . 42

3.6. Dependencia del módulo de bulk $(B)$ con el número atómico $(Z)$ del átomo $X$, para los compuestos ternarios $X \mathrm{Fe}_{3} \mathrm{~N}$. . . . . . . . . 43

3.7. Variación respecto el $Z$ del átomo $X$ del a) $M_{f u}$ y b) momento magnético de los átomos $X$ y FeII de los compuestos ternarios $X \mathrm{Fe}_{3} \mathrm{~N}$. . . . 45

3.8. Densidad de estados del a) $\gamma^{\prime}-\mathrm{Fe}_{4} \mathrm{~N}$ y los compuestos ternarios b)

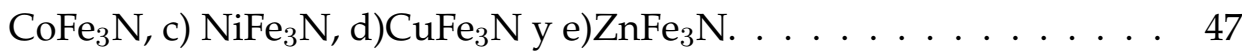

3.9. Dependencia del $<m>$ respecto $Z_{v}$ para los compuestos ternarios

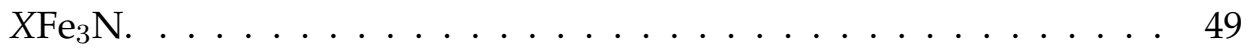

3.10. Valores del a) $B_{h f}$, b) $\delta$ y c) $\varepsilon$ del átomo Fell de los compuestos $X \mathrm{Fe}_{3} \mathrm{~N}$, en función del $\mathrm{Z}$ del átomo $X . \ldots \ldots$

3.11. Curvas de energía total - parámetro de red del compuesto $\mathrm{ScFe}_{3} \mathrm{~N}$. . 52

3.12. Intercepción de las curvas presión de la entalpía para los casos FM y $\mathrm{NM}$ del $\mathrm{ScFe}_{3} \mathrm{~N}$ con estructura $P m \overline{3} m \ldots \ldots$. . . . . . . . . . . 53

3.13. Superficie energética para los diferentes valores de $a$ y $c / a$ indicando el valor óptimo de $c / a$ para el caso de los compuestos ternarios a) $\left.\mathrm{TiFe}_{3} \mathrm{~N}_{\mathrm{y}} \mathrm{b}\right) \mathrm{CrFe}_{3} \mathrm{~N} \ldots \ldots \ldots \ldots \ldots \ldots \ldots$

3.14. Dependencia de $c / a$ respecto del a) $Z$ y b) $r_{m e t}$ del átomo $X$ para los compuestos ternarios $\mathrm{XFe}_{3} \mathrm{~N} \ldots \ldots \ldots \ldots$

3.15. Curva energía - volumen para los compuestos ternarios a) $\mathrm{TiFe}_{3} \mathrm{~N}$, b) $\left.\mathrm{VFe}_{3} \mathrm{~N}, \mathrm{c}\right) \mathrm{CrFe}_{3} \mathrm{~N}$ y d) $\mathrm{MnFe}_{3} \mathrm{~N} \ldots \ldots \ldots \ldots$

3.16. Variación del a) $M_{f u} \mathrm{y}$ b) momento magnético por átomo en función del $Z$ del átomo $X$ de los compuestos ternarios $X \mathrm{Fe}_{3} \mathrm{~N}$ con estructura

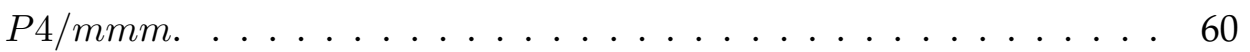

3.17. Variación del a) $M_{f u} \mathrm{y}$ b) momento magnético por átomo en función del $Z$ del átomo $X$ de los compuestos ternarios $X \mathrm{Fe}_{3} \mathrm{~N}$ con estructura

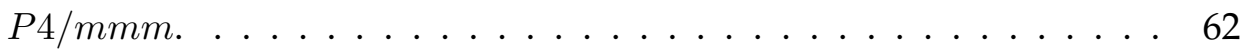

3.18. Valores de $<m>$ en función de $Z_{v}$ obtenido por los cálculos ab-initio y con el modelo SPF. . . . . . . . . . . . . . . . . . . . 63 
3.19. Variación del a) $B_{h f}$, b) $\delta$ y c) $\varepsilon$ en función del $Z$ del átomo $X$ para los compuestos ternarios $\mathrm{XFe}_{3} \mathrm{~N}$ con estructura $\mathrm{P} 4 / \mathrm{mmm}$. . . . . . . 65

3.20. Curvas energía total - volumen para el compuesto $\mathrm{MnFe}_{3} \mathrm{~N}$ en las estructuras cristalinas $P m \overline{3} m$ y $P 4 / m m m$ y en las configuraciones magnéticas NM, AF, FE y FM. . . . . . . . . . . . . . . . 66

3.21. Variación de la energía total, respecto del estado AF, en función del $M_{f u}$ del compuesto $\mathrm{MnFe}_{3} \mathrm{~N}$ para las estructuras cristalinas a) $P m \overline{3} m$ y b) $P 4 / m m m$. . . . . . . . . . . . . . . . . . . . . . 69

3.22. Diferencia de energía $(\Delta E)$ entre las dos fases cristalina. . . . . . . 72

3.23. Curvas energía total - volumen para los compuestos ternarios $X \mathrm{Fe}_{3} \mathrm{~N}$. 73

3.24. Evolución del parámetro de red de los compuestos $X \mathrm{Fe}_{3} \mathrm{~N}$, en función del número atómico $(Z)$ del átomo $X \ldots$. . . . . . . . . . . 74

3.25. Parámetro de red de equilibrio $(a)$ de los compuestos $X \mathrm{Fe}_{3} \mathrm{~N}$ en función del radio metálico $\left(r_{m e t}\right)$ del átomo X. . . . . . . . . . . . 75

3.26. Dependencia con $Z$ del a) $M_{f u}$ obtenidos, junto con valores experimentales (Tabla 2.4) y teóricos (Tabla 2.6), b) momento magnético por átomo. . . . . . . . . . . . . . . . . 77

3.27. Densidad de estados total y por átomo del compuesto ternario a) $\mathrm{RuFe}_{3} \mathrm{~N}$, b) $\left.\left.\mathrm{RhFe}_{3} \mathrm{~N}, \mathrm{c}\right) \mathrm{PdFe}_{3} \mathrm{~N}, \mathrm{~d}\right) \mathrm{AgFe}_{3} \mathrm{~N}$ y e)CdFe ${ }_{3} \mathrm{~N} \ldots \ldots . . . . . . .79$

3.28. Variación del a) $B_{h f}$, b) $\delta$ y c) $\varepsilon$ del átomo de Fe en función del $Z$ del átomo $X \ldots \ldots \ldots \ldots \ldots \ldots$. . . . . . . . . . . . . . 81

4.1. Variación de la energía total, respecto al estado $P 4 / \mathrm{mmm}$-FE, de los compuestos $\mathrm{Mn}_{x} \mathrm{Fe}_{4-x} \mathrm{~N}$ para las estructuras $P m \overline{3} m$ y $P 4 / m m m$ con diferentes configuraciones magnéticas. . . . . . . . . . . . . 86

4.2. Variación del parámetro de red de equilibrio $(a)$ en función de $x$ para las distintas estructuras cristalinas y magnéticas del compuesto $\mathrm{Mn}_{x} \mathrm{Fe}_{4-x} \mathrm{~N} \ldots \ldots \ldots \ldots \ldots \ldots \ldots \ldots$

4.3. Densidad de estados totales y por átomo de los compuestos a) $\mathrm{Mn}_{0.250} \mathrm{Fe}_{3.750} \mathrm{~N}$, b) $\mathrm{Mn}_{0.500} \mathrm{Fe}_{3.500} \mathrm{~N}$ y c) $\mathrm{Mn}_{0.750} \mathrm{Fe}_{3.250} \mathrm{~N} \ldots \ldots . \ldots . \ldots . . \ldots 91$ 
4.4. Diferencia de las densidades de carga up y down para los compuestos a) $\left.\mathrm{Mn}_{0.125} \mathrm{Fe}_{3.875} \mathrm{~N}, \mathrm{~b}\right) \mathrm{Mn}_{0.500} \mathrm{Fe}_{3.500} \mathrm{~N}$ y c) $\mathrm{Mn}_{0.875} \mathrm{Fe}_{3.125} \mathrm{~N} \ldots \ldots . . . .992$

4.5. Variación con $x$ de la a) $M_{f u}$ de las estructuras $P m \overline{3} m$ y $P 4 / m m m$ para los estados FE y FM, b) momento magnético de los átomos de Fe de la configuración de mínima energía $P 4 / m m m$-FE. . . . . . . . 94

4.6. Dependencia con $x$ del a) $B_{h f}$, b) $\delta$ y c) $\varepsilon$ de los átomos FeI y FeII de los compuestos $\mathrm{Mn}_{x} \mathrm{Fe}_{4-x} \mathrm{~N} \ldots \ldots \ldots \ldots$. . . . . . . . . 96

4.7. Dependencia lineal entre el momento magnético $(m)$ y el campo hiperfino $\left(B_{h f}\right)$ de los átomos FeI de los compuestos ternarios $\mathrm{Mn}_{x} \mathrm{Fe}_{4-x} \mathrm{~N} .97$

4.8. Variación del parámetro de red $(a)$ con la concentración $(x)$ para los compuestos a) $\mathrm{Co}_{x} \mathrm{Fe}_{4-x} \mathrm{~N}$, junto con los valores experimentales obtenidos de [148], b) $\mathrm{Ni}_{x} \mathrm{Fe}_{4-x} \mathrm{~N}$, junto con los valores experimentales reportados $[103-110] \ldots \ldots$. . . . . . . . . . . . . . . 100

4.9. Dependencia lineal del módulo de mulk (B) con $x$ para los compuestos $\mathrm{Co}_{x} \mathrm{Fe}_{4-x} \mathrm{~N} \mathrm{y} \mathrm{Ni}_{x} \mathrm{Fe}_{4-x} \mathrm{~N}$. . . . . . . . . . . . . . . . . . . 101

4.10. Dependencia del $M f u$ con $x$ para los compuestos a) $\mathrm{Co}_{x} \mathrm{Fe}_{4-x} \mathrm{~N}$ y b) $\mathrm{Ni}_{x} \mathrm{Fe}_{4-x} \mathrm{~N}$. En el caso b) se pudo comparar con los valores experimentales [119,126](cuadrados azules), [106] (rombos rojos) y otros valores experimentales recopilador [104,105,107-109,120-122] (triángulos verdes) . . . . . . . . . . . . . . . . . . . . . 103

4.11. Valor del momento magnético de los átomos FeI y FeII de los compuestos $\mathrm{Co}_{x} \mathrm{Fe}_{4-x} \mathrm{~N}_{\text {y Ni}} \mathrm{Ne}_{4-x} \mathrm{~N}$, para los diferentes valores de $x . \quad$. 104

4.12. Variación del $B_{h f}$ con $x$ de los átomos FeI y FeII para los compuestos a) $\mathrm{Co}_{x} \mathrm{Fe}_{4-x} \mathrm{~N}$ y b) $\mathrm{Ni}_{x} \mathrm{Fe}_{4-x} \mathrm{~N}$, junto con diferentes valores experimentales recopilados de la bibliografía. Símbolos azules y verdes representan los valores de $B_{h f}$ experimental de los FeI y FeII, respectivamente. . . . . . . . . . . . . . . 105 
4.13. Variación del $\delta$ con $x$ de los átomos FeI y Fell para los compuestos a) $\mathrm{Co}_{x} \mathrm{Fe}_{4-x} \mathrm{~N}$ y b) $\mathrm{Ni}_{x} \mathrm{Fe}_{4-x} \mathrm{~N}$, junto con diferentes valores experimentales recopilados de la bibliografía. Símbolos azules y verdes representan los valores de $\delta$ experimental de los FeI y FeII, respectivamente. . . . . . . . . . . . . . . . . 107

4.14. Dependencia lineal del $\varepsilon$ con $x$ de los átomos FeII para los compues-

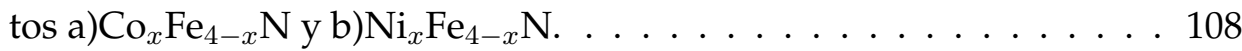

5.1. Curvas energía total - volumen de las estructuras $P m \overline{3} m$ y $P 4 / m m m$ de los compuestos a)AlFe $\left.{ }_{3} \mathrm{~N}, \mathrm{~b}\right) \mathrm{GaFe}_{3} \mathrm{~N}$ y c)InFe ${ }_{3} \mathrm{~N}$. . . . . . . . . . 113

5.2. Variación de $\Delta E$ en función del $M_{f u}$ para a) $\mathrm{AlFe}_{3} \mathrm{~N}$, b) $\mathrm{GaFe}_{3} \mathrm{~N}$ y

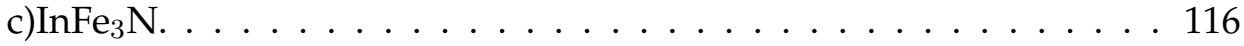

5.3. Variación del volumen relativo $\omega$ respecto de $M_{f u}$ para los compuestos $\mathrm{AlFe}_{3} \mathrm{~N}, \mathrm{GaFe}_{3} \mathrm{~N}$ y InFe $\mathrm{InF}_{3} \mathrm{~N} \ldots \ldots . . \ldots 117$

5.4. Variación del parámetro de red $(a)$ con $x$ (círculos negros) para los compuestos a) $\left.\mathrm{Al}_{x} \mathrm{Fe}_{4-x} \mathrm{~N}, \mathrm{~b}\right) \mathrm{Ga}_{x} \mathrm{Fe}_{4-x} \mathrm{~N}$, junto a los valores experimentales de [155] (símbolos rojos) y [149] (símbolos azules), c) $\operatorname{In}_{x} \mathrm{Fe}_{4-x} \mathrm{~N}$, junto a los valores experimentales de [152] (símbolos rojos). . . . . . 121

5.5. Densidad de estados electrónicos (DOS) de los compuestos a) $\mathrm{Al}_{0.500} \mathrm{Fe}_{3.500} \mathrm{~N}$, b) $\mathrm{Ga}_{0.500} \mathrm{Fe}_{3.500} \mathrm{~N}$ y c) $\operatorname{In}_{0.500} \mathrm{Fe}_{3.500} \mathrm{~N}$, totales y por átomo. . . . . . . . 127

5.6. Densidad de estados electrónicos (DOS) de los compuestos a) $\mathrm{Al}_{1.000} \mathrm{Fe}_{3.000} \mathrm{~N}$, b) $\mathrm{Ga}_{1.000} \mathrm{Fe}_{3.000} \mathrm{~N}$ y c) $\operatorname{In}_{1.000} \mathrm{Fe}_{3.000} \mathrm{~N}$, totales y por átomo. . . . . . . . 128

5.7. Carga del orbital $3 d$-up y $3 d$-down de los átomos FeI y FeII en función de $x$ para los compuestos a) $\left.\mathrm{Al}_{x} \mathrm{Fe}_{4-x} \mathrm{~N}, \mathrm{~b}\right) \mathrm{Ga}_{x} \mathrm{Fe}_{4-x} \mathrm{~N}$ y c) $\operatorname{In}_{x} \mathrm{Fe}_{4-x} \mathrm{~N} .130$

5.8. Momentos magnéticos de los átomos $M, \mathrm{Fe} I$ y FeII en función de $x$, para los compuestos a) $\left.\mathrm{Al}_{x} \mathrm{Fe}_{4-x} \mathrm{~N}, \mathrm{~b}\right) \mathrm{Ga}_{x} \mathrm{Fe}_{4-x} \mathrm{~N}$ y c) $\operatorname{In}_{x} \mathrm{Fe}_{4-x} \mathrm{~N}$. . . . 132

5.9. Dependencia del momento magnético de los FelI con la cantidad de átomos $M$ como $n n n$ para los compuestos a) $\left.\mathrm{Al}_{x} \mathrm{Fe}_{4-x} \mathrm{~N}, \mathrm{~b}\right) \mathrm{Ga}_{x} \mathrm{Fe}_{4-x} \mathrm{~N}$ y c) $\operatorname{In}_{x} \mathrm{Fe}_{4-x} \mathrm{~N} \ldots \ldots \ldots \ldots \ldots \ldots \ldots$ 
5.10. Variación del $M_{f u}$ respecto de $x$ para a) $\left.\mathrm{Al}_{x} \mathrm{Fe}_{4-x} \mathrm{~N}, \mathrm{~b}\right) \mathrm{Ga}_{x} \mathrm{Fe}_{4-x} \mathrm{~N}$, círculos verdes y rojos son datos experimentales de [151] y [155], respectivamente, c) $\operatorname{In}_{x} \mathrm{Fe}_{4-x} \mathrm{~N}$, círculos rojos son datos experimentales

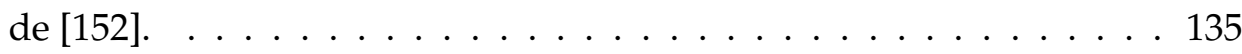

5.11. Dependencia del $B_{h f}$ con $x$ de los átomos FeI y FeII de los compuestos a) $\left.\mathrm{Al}_{x} \mathrm{Fe}_{4-x} \mathrm{~N}, \mathrm{~b}\right) \mathrm{Ga}_{x} \mathrm{Fe}_{4-x} \mathrm{~N} \mathrm{y} \mathrm{c)} \operatorname{In}_{x} \mathrm{Fe}_{4-x} \mathrm{~N} . \ldots \ldots 137$

5.12. Dependencia del $B_{h f}$ con el momento magnético de los átomos FeII de los compuestos $M_{x} \mathrm{Fe}_{4-x} \mathrm{~N}$. . . . . . . . . . . . . . . . 138

5.13. Dependencia del $B_{h f}$ de los FelI con el número de átomos $M n n n$ para los compuestos a) $\left.\mathrm{Al}_{x} \mathrm{Fe}_{4-x} \mathrm{~N}, \mathrm{~b}\right) \mathrm{Ga}_{x} \mathrm{Fe}_{4-x} \mathrm{~N}$ y c) $\operatorname{In}_{x} \mathrm{Fe}_{4-x} \mathrm{~N}$. . . 140

5.14. Variación del $\delta$ de los FeII en función del número de átomos $M n n n$ para los compuestos a) $\left.\mathrm{Al}_{x} \mathrm{Fe}_{4-x} \mathrm{~N}, \mathrm{~b}\right) \mathrm{Ga}_{x} \mathrm{Fe}_{4-x} \mathrm{~N}$ y c) $\operatorname{In}_{x} \mathrm{Fe}_{4-x} \mathrm{~N}$. . . . 142

5.15. Variación del $\varepsilon$ de los FeII en función del número de átomos $M n n n$ para los compuestos a) $\left.\mathrm{Al}_{x} \mathrm{Fe}_{4-x} \mathrm{~N}, \mathrm{~b}\right) \mathrm{Ga}_{x} \mathrm{Fe}_{4-x} \mathrm{~N}$ y c) $\operatorname{In}_{x} \mathrm{Fe}_{4-x} \mathrm{~N}$. . . 143 


\section{Lista de Tablas}

2.1. Valores experimentales del parámetro $(a)$ de red del $\gamma^{\prime}-\mathrm{Fe}_{4} \mathrm{~N}$ para diferentes tipos de muestra, obtenidos de la bibliografía. . . . . . . . 20

2.2. Valores experimentales del momento magnético $\left(\mathrm{M}_{f u}\right) \operatorname{del} \gamma^{\prime}-\mathrm{Fe}_{4} \mathrm{~N}$, momento magnético por átomos $(m)$, campo hiperfino $\left(\mathrm{B}_{h f}\right)$, corrimiento isomérico $(\delta)$ y desdoblamiento cuadrupolar $(\varepsilon)$ de los átomos de Fe del $\gamma^{\prime}-\mathrm{Fe}_{4} \mathrm{~N} \ldots \ldots \ldots 21$

2.3. Valores experimentales del parámetro de red $(a)$ de los compuestos ternarios $\mathrm{XFe}_{3} \mathrm{~N}$ existentes en la bibliografía. . . . . . . . . . . . 22

2.4. Valores experimentales obtenidos de la bibliografía del momento magnético total $\left(M_{f u}\right)$ de los compuestos ternarios $X \mathrm{Fe}_{3} \mathrm{~N}$, momento magnético por átomo $(m)$ junto con el campo hiperfino $\left(B_{h f}\right)$, corrimiento isomérico $(\delta)$ y desdoblamiento cuadrupolar $(\varepsilon)$ de los átomos de Fe.

2.5. Valores teóricos recopilados de la bibliografía del parámetro de red (a) y el módulo de bulk (B) de los compuestos ternarios $\mathrm{XFe}_{3} \mathrm{~N}$ con estructura $P m \overline{3} m \ldots \ldots \ldots \ldots \ldots \ldots \ldots \ldots$

2.6. Valores teóricos del momento magnético por fórmula unidad $\left(\mathrm{M}_{f u}\right)$, por átomo $(m)$ y parámetros hiperfinos del átomo de Fe de los compuestos $\mathrm{XFe}_{3} \mathrm{~N}$ con estructura cristalina $\mathrm{Pm} \overline{3} m . \ldots \ldots$

2.7. Razón entre la cantidad de átomos $M$ y FeI $(M: \mathrm{Fe} I)$ en la supercelda $2 \times 2 \times 2$ de $\gamma^{\prime}-\mathrm{Fe}_{4} \mathrm{~N}$ para obtener diferentes valores de concentración $x$ en los compuestos $M_{x} \mathrm{Fe}_{4-x} \mathrm{~N}$, con sus respectivas configuraciones no equivalente. . . . . . . . . . . . . . . 29 
3.1. Volumen de equilibrio $\left(V_{0}\right)$ y el módulo de bulk $(B)$ obtenidos del ajuste con la ecuación de estado de Birch-Murnaghan, para ambas estructuras cristalinas del compuesto ternario $X \mathrm{Fe}_{3} \mathrm{~N}$. Además, la diferencia de la energía total $(\Delta E)$ entre ambas estructuras en su res-

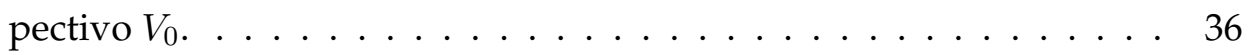

3.2. Valores del momento magnético por fórmula unidad $\left(M_{f u}\right)$ y de los momentos magnéticos por átomo de los compuestos ternarios XFeN obtenidos en el presente trabajo. . . . . . . . . . . . . . 44

3.3. Valores del momento magnético por fórmula unidad $\left(M_{f u}\right)$ y de los momentos magnéticos por átomo de los compuestos ternarios XFeN obtenidos en el presente trabajo. . . . . . . . . . . . . . . 5

3.4. Parámetro de red de equilibrio del $\mathrm{ScFe}_{3} \mathrm{~N}$, para las dos estructuras cristalinas en las fase magnética (FM) y no magnética (NM), junto con la variación de la energía total respecto del estado $\operatorname{Pm} \overline{3} m$ no magnético. . . . . . . . . . . . . . . . 52

3.5. Volumen $\left(V_{0}\right)$, parámetros de red $(a$ y $c$ ) de equilibrio y módulo de bulk $(B)$ de los compuestos ternarios $\mathrm{XFe}_{3} \mathrm{~N} \ldots \ldots \ldots 7$

3.6. Momento magnético por fórmula unidad $\left(M_{f u}\right)$ y momentos magnéticos por átomo de los compuestos ternarios $\mathrm{XFe}_{3} \mathrm{~N}$ con estructura $P 4 / m m m \ldots \ldots \ldots \ldots \ldots \ldots \ldots$

3.7. Parámetro de red de equilibrio $(a)$, módulo de bulk $(B)$ y variación de la energía respecto del estado $P 4 / m m m$-FE. . . . . . . . . . . 68

3.8. Valores del $M_{f u}$, momento magnético por átomo y campo hiperfino $\left(B_{h f}\right)$ de los átomos de Fe del compuesto $\mathrm{MnFe}_{3} \mathrm{~N}$, para las estructuras cristalinas $P m \overline{3} m$ y $P 4 / m m m$ en las fases magnéticas AF, FE y FM. ...................... 70

3.9. Volumen $\left(V_{0}\right)$, parámetro de red $(a)$ y módulo de bulk $(B)$ de equilibrio, de los compuestos $\mathrm{XFe}_{3} \mathrm{~N}$ en la fase cristalina $\operatorname{Pm} \overline{3} m$. . . . . 74

3.10. Valores calculados del $M_{f u}$ y momento magnético por átomo. . . . . 76 
3.11. Valores calculados del $B_{h f}, \delta$ y $\varepsilon$ del átomo FeII para los diferentes compuestos ternarios $\mathrm{XFe}_{3} \mathrm{~N} \ldots \ldots \ldots \ldots$. . . . . . . . 80

4.1. Configuración y parámetro de red de equilibrio $(a)$ de los compuestos $\mathrm{Mn}_{x} \mathrm{Fe}_{4-x} \mathrm{~N}$ correspondiente a la configuración de mínima energía

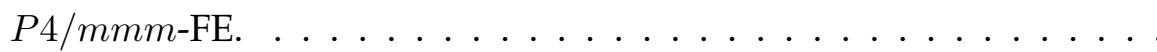

4.2. Diferentes tipos de átomos FeI y FeII respecto a sus primeros $(n n)$ y segundos vecinos $(n n n)$, para las diferentes concentraciones $(x)$ de los compuestos $\mathrm{Mn}_{x} \mathrm{Fe}_{4-x} \mathrm{~N}$. El número en paréntesis representa la cantidad de dicho tipo de átomo. . . . . . . . . . . . . . . . 89

4.3. Configuración de mínima energía, parámetro de red de equilibrio $(a)$ y su respectivo módulo de bulk (B) de los compuestos $\mathrm{Co}_{x} \mathrm{Fe}_{4-x} \mathrm{~N} \mathrm{y}$ $\mathrm{Ni}_{x} \mathrm{Fe}_{4-x} \mathrm{~N} \ldots \ldots \ldots \ldots \ldots \ldots \ldots \ldots$

5.1. Valores de $a, M_{f u} \mathrm{y}$ momento magnético por átomo $(m)$ obtenidos de los cálculos ab-initio junto con otros valores teóricos y experimentales. 114

5.2. Valores de las barreras de energía (mRy) del punto $\mathrm{A}$ hacia $\mathrm{B}(\mathrm{A} \rightarrow \mathrm{B})$

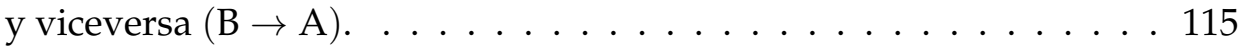

5.3. Configuraciones de mínima energía correspondiente a cada valor de $x .118$

5.4. Configuraciones de mínima energía correspondiente a cada valor de $x .119$

5.5. Diferentes tipos de átomos FeI y FeII de acuerdo a sus primeros $(n n)$ y segundos vecinos $(n n n)$ correspondiente a los distintos valores de $(x)$ de los compuestos $M_{x} \mathrm{Fe}_{4-x} \mathrm{~N}$ ( $M=\mathrm{Al}$ o In). El número en paréntesis representa la cantidad de dicho tipo de átomo. . . . . . . . . . . . . . . . . . . . . . . . 124

5.6. Diferentes tipos de átomos FeI y FeII de acuerdo a sus primeros $(n n)$ y segundos vecinos $(n n n)$ correspondiente a los distintos valores de $(x)$ de los compuestos $M_{x} \mathrm{Fe}_{4-x} \mathrm{~N}(M=\mathrm{Ga})$. El número en paréntesis representa la cantidad de dicho tipo de átomo. . . . . . . . . . . . . . . . . . . 125

5.7. Valores del $M_{f u}$ en función de $x$ para los compuestos $M_{x} \mathrm{Fe}_{4-x} \mathrm{~N}$. . . 134 
5.8. Valores del $B_{h f}(\mathrm{~T}), \delta(\mathrm{mm} / \mathrm{s})$ y $\varepsilon(\mathrm{mm} / \mathrm{s})$ de los diferentes átomos de Fe de los compuestos $M_{x} \mathrm{Fe}_{4-x} \mathrm{~N} \ldots \ldots \ldots \ldots$. . . . . . . . . 136 


\section{Introducción}

AS aleaciones de hierro-nitrógeno han sido objeto de estudio en las últimas
décadas debido a su importancia tanto científica como tecnológica, donde sus aplicaciones abarca desde el recubrimiento de aceros, con el fin de mejorar sus propiedades mecánicas tales como dureza, resistencia a la corrosión, fractura, mejoramiento contra el desgaste, etc [1-5], hasta aplicaciones magnéticas [6-8]. Con tal fin, han sido propuestos distintos sistemas binarios, ternarios o estructuras más complejas [6-9] a partir de diferentes estudios experimentales o teóricos. Uno de este tipo de aleaciones de hierro-nitrógeno, es el sistema binario $\gamma^{\prime}-\mathrm{Fe}_{4} \mathrm{~N} \gamma^{\prime}-\mathrm{Fe}_{4} \mathrm{~N}$, el cual fue inicialmente estudiado por P.H. Emmett et al. [10] motivado por sus posibles aplicaciones como un catalizador en la producción de amonio. Consecuentemente, este compuesto, debido a sus propiedades magnéticas que presenta, fue propuesto como un buen candidato para nuevas aplicaciones tecnológicas relacionado a la fabricación de dispositivos de almacenamiento magnético de alta densidad [11-14]. Motivo por el cual ha sido objeto de estudio tanto desde el punto de vista experimental como teórico.

En la presente tesis, se estudiarán diferentes compuestos ternarios alternativos obtenidos a partir de la sustitución de ciertos átomos de $\mathrm{Fe}$ del $\gamma^{\prime}-\mathrm{Fe}_{4} \mathrm{~N}$ por otro átomo $(M)$, como puede ser metales $3 d, 4 d$ o metales del bloque $p$ de la Tabla Periódica. Con tal fin, se ha usado uno de los métodos teóricos más sofisticados y ampliamente utilizados en el estudio y/o caracterización de nuevos materiales, tal como lo son los cálculos de primeros principios, o ab-initio, basados en la Teoría de 
la Funcional Densidad. Estos modelos nos permitirá determinar el sitio preferencial de sustitución del átomo $M$ en el compuesto $\gamma^{\prime}-\mathrm{Fe}_{4} \mathrm{~N}$, calcular el parámetro de red de equilibrio, el módulo de bulk como también hacer una caracterización de las propiedades magnéticas e hiperfinas de estos compuestos ternarios. La presente tesis se dividirá de la siguiente manera:

CAPÍTULO 1: Se abordara de forma concreta y muy referenciada la Teoría de la Funcional Densidad, como también el método autoconsistente Full Potential Augmented Plane Wave (FP-LAPW). Aquí, se describirá las principales características que hicieron posible la implementación computacional de dicho método en el código Wien2K.

CAPÍtulo 2: Estará dedicado inicialmente a la recopilación de los diferentes datos experimentales y teóricos, obtenidos de la bibliografía vigente, de las propiedades estructurales, magnéticas e hiperfinas de los compuestos ternarios estudiados. Esto nos servirá como una base de datos con los cuales serán comparados con los resultados obtenidos en el desarrollo del presente trabajo. Además, también se describen los detalles computacionales que se estandarizaron en todos los cálculos realizados, como el tipo de aproximaciones utilizadas para la obtención y / o procesamiento de los resultados.

CAPÍtulo 3: Se estudiarán los compuestos ternarios $\mathrm{XFe}_{3} \mathrm{~N}$ donde $X$ es un metal $3 d$ (Sc, Ti, V, Cr, Mn, Co, Ni, Cu, Zn) o $4 d(\mathrm{Ru}, \mathrm{Rh}, \mathrm{Pd}, \mathrm{Ag}, \mathrm{Cd})$, donde se analizará primero el sitio preferencial de sustitución del átomo $M$ en el compuesto $\gamma^{\prime}-\mathrm{Fe}_{4} \mathrm{~N}$ y cómo varia el volumen y/o parámetro de red de equilibrio de los nuevos compuestos ternarios, los cuales se contrastaran con los valores experimentales obtenidos de la bibliografía. Además se analizarán los cambios que dicha sustitución ocasiona en la estructura electrónica del $\gamma^{\prime}-\mathrm{Fe}_{4} \mathrm{~N}$, al formar los nuevos compuestos ternarios, y como esto se verá reflejado en la variación de las propiedades magnéticas e hiperfinas, las cuales se compararan con valores experimentales. Para el caso 
de las propiedades magnéticas, se comparará también con el modelo propuesto por Slater-Pauling-Friedel.

CAPÍtulo 4: El presente capítulo estará dedicado a la caracterización de los compuestos ternarios $M_{x} \mathrm{Fe}_{4-x} \mathrm{~N}(0 \leq x \leq 1)$ cuando $M$ es $\mathrm{Mn}$, Co o $\mathrm{Ni}$, se analizará la dependencia del parámetro de red con la concentración $(x)$ del átomo $M$, como también, los cambios en las propiedades magnéticas e hiperfinas que ocasiona la sustitución de átomos de Fe por átomos $M$.

CAPÍtulo 5: Estará dedicado inicialmente al estudio del estado fundamental estructural y magnético de los compuestos ternarios $M \mathrm{Fe}_{3} \mathrm{~N}$, donde $M$ es $\mathrm{Al}, \mathrm{Ga}$ o In, para lo cual se realizarán cálculos fijando el momento magnético total de cada compuesto. Una vez determinado el estado fundamental de cada compuesto, se procederá a estudiar la dependencia del parámetro de red de equilibrio con la concentración ( $x$ ) del átomo $M$ en los compuestos $M_{x} \mathrm{Fe}_{4-x} \mathrm{~N}$. Se investigara el tipo de interacción magnética existente entre los átomos $M$ y los de $\mathrm{Fe}$, y como esto ocasiona cambios en las propiedades magnéticas de estos compuestos. Se determinara como influye la variación del momento magnético de ciertos átomos de Fe, como consecuencia de la modificación de su entorno y como se modificarán sus propiedades hiperfinas. 


\section{Capítulo 1}

\section{Fundamento Teórico}

\subsection{Sistema de Muchos Cuerpos}

Desde el punto de vista microscópico, un sólido puede ser representado como una colección de núcleos $(n)$ cargados positivamente y electrones $(e)$ cargados negativamente. Los núcleos y los electrones son tratados como cargas puntuales que interactuan electromagnéticamente, entonces el Hamiltoniano no relativista (en unidades de Ry) para este sistema de muchos cuerpos será [15]:

$$
\begin{aligned}
\hat{\mathcal{H}}= & -\frac{\hbar^{2}}{2} \sum_{i} \frac{\nabla_{\mathbf{R}_{i}}^{2}}{M_{i}}-\frac{\hbar^{2}}{2} \sum_{i} \frac{\nabla_{\mathbf{r}_{i}}^{2}}{m_{e}}-\frac{1}{4 \pi \varepsilon_{0}} \sum_{i, j} \frac{e^{2} Z_{i}}{\left|\mathbf{R}_{i}-\mathbf{r}_{i}\right|} \\
& +\frac{1}{8 \pi \varepsilon_{0}} \sum_{i \neq j} \frac{e^{2}}{\left|\mathbf{r}_{i}-\mathbf{r}_{j}\right|}+\frac{1}{8 \pi \varepsilon_{0}} \sum_{i \neq j} \frac{e^{2} Z_{i} Z_{j}}{\left|\mathbf{R}_{i}-\mathbf{R}_{j}\right|}
\end{aligned}
$$

Donde, $M_{i}$ es la masa del núcleo ubicado en $\mathbf{R}_{i}$ mientras que $m_{e}$ es la masa de los electrones ubicados en $\mathbf{r}_{i}$. El primer y segundo termino de (1.1) corresponden al operador de la energía cinética de los núcleos y de los electrones, respectivamente. Los demás términos describen la interacción Coulombiana entre los electrones y los núcleos, entre los electrones y entre los núcleos, respectivamente.

Considerando que $|\Psi(\mathbf{R}, \mathbf{r})\rangle$ representa la función de onda de este sistema, entonces la ecuación de Schrödinguer correspondiente será:

$$
\hat{\mathcal{H}}|\Psi(\mathbf{R}, \mathbf{r})\rangle=E|\Psi(\mathbf{R}, \mathbf{r})\rangle
$$

donde $\mathbf{R}=\left\{\mathbf{R}_{i} \mid i=1, \cdots, N_{n}\right\}$ y $\mathbf{r}=\left\{\mathbf{r}_{i} \mid i=1, \cdots, N_{e}\right\}$ representan las posiciones de $\operatorname{los} N_{n}$ núcleos y los $N_{e}$ electrones, respectivamente. Desafortunadamente 
la ecuación (1.2) es imposible de resolver en forma analítica debido a su alto grado de complejidad, pero es posible hacerlo numéricamente, para lo cual es necesario introducir algunas aproximaciones.

\subsubsection{Aproximación de Born-Oppenheimer}

A pesar que las fuerzas electromagnéticas actuando entre electrones y núcleos son de magnitudes comparables, el movimiento de los electrones $\left(\approx 10^{6} \mathrm{~m} / \mathrm{s}\right)$ es considerablemente más rápido que el de los núcleos $\left(\approx 10^{3} \mathrm{~m} / \mathrm{s}\right)$, esto es una consecuencia de la gran diferencia entre la masa del electrón y la de los núcleos. Por lo tanto, los núcleos se mueven más lentamente respecto a la escala de velocidades de los electrones, pudiéndose considerarlos como fijos, por lo tanto se puede despreciar la contribución de la energía cinética de los núcleos en (1.1). Así, se tiene que el Hamiltoniano (1.1) puede ser escrito como:

$$
\hat{\mathcal{H}}=\hat{\mathcal{T}}-\hat{\mathcal{V}}_{\text {ext }}+\hat{\mathcal{V}}+\hat{\mathcal{V}}_{N N}
$$

donde:

$$
\begin{aligned}
\hat{\mathcal{T}} & =-\frac{\hbar^{2}}{2} \sum_{i} \frac{\nabla_{\mathbf{r}_{i}}^{2}}{m_{e}} \\
\hat{\mathcal{V}}_{e x t} & =\frac{1}{4 \pi \varepsilon_{0}} \sum_{i, j} \frac{e^{2} Z_{i}}{\left|\mathbf{R}_{i}-\mathbf{r}_{i}\right|} \\
\hat{\mathcal{V}} & =\frac{1}{8 \pi \varepsilon_{0}} \sum_{i \neq j} \frac{e^{2}}{\left|\mathbf{r}_{i}-\mathbf{r}_{j}\right|} \\
\hat{\mathcal{V}}_{N N} & =\frac{1}{8 \pi \varepsilon_{0}} \sum_{i \neq j} \frac{e^{2} Z_{i} Z_{j}}{\left|\mathbf{R}_{i}-\mathbf{R}_{j}\right|}
\end{aligned}
$$

El hamiltoniano (1.3) corresponde a un movimiento de electrones inmersos en un potencial externo producido por los núcleos, donde $\hat{\mathcal{V}}_{N N}$ denota la interacción entre los núcleos, la cual es considerada una cantidad constante que no influye en la función de onda.

Luego, el hamiltoniano (1.3) puede ser expresado como:

$$
\begin{aligned}
\hat{\mathcal{H}} & =\hat{\mathcal{H}}_{e}+\hat{\mathcal{V}}_{N N} \\
\hat{\mathcal{H}}_{e} & =\hat{\mathcal{T}}+\hat{\mathcal{V}}_{\text {ext }}+\hat{\mathcal{V}}
\end{aligned}
$$


donde, $\hat{\mathcal{H}}_{e}$ es denominado el hamiltoniano electrónico, $\hat{\mathcal{T}}$ representa el operador energía cinética de los electrones, $\hat{\mathcal{V}}_{\text {ext }}$ el operador de interacción electrón-núcleo y $\hat{\mathcal{V}}$ es el operador de repulsión electrónica. Por lo tanto, los electrones y los núcleos pueden ser tratados de forma independiente, a este desacoplamiento del movimiento electrónico y nuclear se conoce como la Aproximación de Born-Oppenheimer [16].

Hasta este punto, a pesar de la aproximación previa, el desarrollo cuántico de un sistema de muchos cuerpos es aún muy complejo de resolver, conllevando a realizar más aproximaciones relacionadas con la manera de solucionar (1.4). Una de ellas es la propuesta por Hartree-Fock (HF) [17-19], en donde se plantea que la solución de (1.4) puede ser escrita como un determinante de Slater [19], conllevando a una solución exacta. El inconveniente del método de HF radica en su alta complejidad numérica para aplicarlo a un sólido, siendo generalmente usado para el estudio de moléculas y/o sistemas aislados. Otro método de solucionar (1.4) es la Teoría de la Funcional Densidad, la cual será descrita a continuación.

\subsection{Teoría de la Funcional Densidad}

En el año 1927 L. Thomas y E. Fermi (TF) [20, 21], desarrollaron una teoría cuántica para describir un gas de electrones homogéneo, donde proponen que las componentes del Hamiltoniano pueden ser expresados en función de la densidad electrónica. En este desarrollo, TF no consideraron efectos de intercambio y correlación, los cuales fueron posteriormente introducidos por P. Dirac en 1930 [22].

En 1964 P. Hohenberg y W. Khon (H-K) [23] y en 1965 W. Khon y L.J. Sham (KS) [24], basándose en los desarrollos de TF propusieron la denominada Teoría de la Funcional Densidad (DFT, Density Functional Theory). El desarrollo de H-K y K-S se basó esencialmente en la demostración de dos teoremas propuestos por ellos. par Esta teoría muestra cómo se puede pasar de un sistema de muchos cuerpos a un sistema de una simple partícula, con la incorporación de los potenciales de intercambio y correlación. La DFT ha dado lugar a muy importantes desarrollos, 
como son los estudios de primeros principios (ab-initio) aplicado a la caracterización de nuevos materiales.

\subsubsection{Teoremas de Hohenberg-Kohn}

TEOREMA 1 : Cualquier observable de un estado estacionario fundamental no degenerado puede ser calculado, en principio de forma exacta, a partir de la densidad electrónica, $n(\mathbf{r})$, de este estado fundamental.

Es decir, que para cualquier observable $\hat{\mathcal{O}}$, su valor de expectación puede expresarse como una funcional de la densidad electrónica del estado fundamental exacto.

$$
\langle\Psi|\hat{\mathcal{O}}| \Psi\rangle=O[n(\mathbf{r})]
$$

TEOREMA 2 : La densidad exacta del estado fundamental minimiza la funcional de la energía total de un estado no degenerado, la cual es una funcional única de la densidad electrónica $n(\mathbf{r})$.

Para el caso del hamiltoniano $\hat{H}$, la funcional de la energía total $H[n]=E_{V_{e x t}}[n]$ es de la forma:

$$
\begin{aligned}
& E_{V_{e x t}}[n]=\langle\Psi|\hat{\mathcal{T}}+\hat{\mathcal{V}}| \Psi\rangle+\left\langle\Psi\left|\hat{\mathcal{V}}_{e x t}\right| \Psi\right\rangle \\
& E_{V_{e x t}}[n]=F_{H K}[n]+\int n(\mathbf{r}) V_{\text {ext }}(\mathbf{r}) d \mathbf{r}
\end{aligned}
$$

donde $F_{H K}[n]$ es la funcional de densidad universal de Hohenberg-Kohn para cualquier sistema de muchos cuerpos

\subsubsection{Ecuación de Kohn-Sham}

En la DFT a pesar de que la funcional $F_{H K}[n]$ es universal para cualquier sistema, no existe una expresión conocida que la describa. En 1964, Kohn-Sham propusieron reescribir $F_{H K}[n]$ de la siguiente forma [24]:

$$
F_{H K}[n]=T_{0}[n]+V_{H}[n]+V_{x c}[n]
$$


donde $T_{0}[n]$ es la funcional de la energía cinética de un sistema de electrones no interactuantes, $V_{H}[n]$ es la contribución de Hartree, el cual describe la interacción con un potencial promedio producido por los demás electrones, este potencial a pesar de no describir una interacción electrón-electrón, es una buena aproximación para las interacción de electrones. A $V_{x c}[n]$ se le denomina potencial de intercambiocorrelación, $V_{x c}[n]=V_{x}[n]+V_{c}[n]$, la cual contiene las correcciones a la energía total debido por un lado al hecho de considerar el operador $T_{0}[n]$ y por otro lado, el considerar que la interacción entre electrones es Coulombiana [25]. Ahora, asumiendo que se conoce la funcional $V_{x c}[n]$, se tiene que:

$$
E_{V_{e x t}}[n]=T_{0}[n]+V_{H}[n]+V_{x c}[n]+V_{e x t}[n]
$$

La ecuación (1.10) puede ser interpretada como la funcional de energía de un sistema de partículas no interactuantes sometidas a dos potenciales externos, $V_{\text {ext }}[n] \mathrm{y}$ $V_{x c}[n]$, cuyo correspondiente hamiltoniano de Kohn-Sham es:

$$
\begin{aligned}
\hat{\mathcal{H}}_{K S} & =\hat{\mathcal{T}}_{0}+\hat{\mathcal{V}}_{H}+\hat{\mathcal{V}}_{x c}+\hat{\mathcal{V}}_{e x t} \\
& =-\frac{\hbar^{2}}{2 m_{e}} \nabla_{i}^{2}+\frac{e^{2}}{4 \pi \varepsilon_{0}} \int \frac{n(\mathbf{r})}{\left|\mathbf{r}-\mathbf{r}^{\prime}\right|} d \mathbf{r}^{\prime}+\hat{\mathcal{V}}_{x c}+\hat{\mathcal{V}}_{e x t}
\end{aligned}
$$

con el operador de intercambio-correlación expresado como la derivada funcional:

$$
\hat{\mathcal{V}}_{x c}=\frac{\partial V_{x c}[n]}{\partial n}
$$

Ahora, considerando al potencial efectivo $V_{\text {eff }}$ :

$$
V_{e f f}(\mathbf{r})=\frac{e^{2}}{4 \pi \varepsilon_{0}} \int \frac{n(\mathbf{r})}{\left|\mathbf{r}-\mathbf{r}^{\prime}\right|} d \mathbf{r}^{\prime}+\frac{\partial V_{x c}[n]}{\partial n}+V_{e x t}
$$

la ecuación (1.12) puede ser representada como [25]:

$$
\hat{\mathcal{H}}_{K S}=-\frac{\hbar^{2}}{2 m_{e}} \nabla_{i}^{2}+\int n(\mathbf{r}) V_{e f f}(\mathbf{r}) d^{3} \mathbf{r}
$$

La ecuación (1.15) es semejante al Hamiltoniano de una simple partícula sometida en un potencial efectivo, es decir, Kohn-Sham transformaron un problema de muchos cuerpos a un sistema de simples partículas no interactuantes, pero conservando la misma densidad electrónica $n(\mathbf{r})$ en el estado fundamental, donde $n(\mathbf{r})$ 
para un sistema de $N$ electrones puede ser expresado como:

$$
n(\mathbf{r})=\sum_{i=1}^{N}\left\langle\psi_{i}\left(\mathbf{r}^{\prime}\right) \mid \psi_{i}\left(\mathbf{r}^{\prime}\right)\right\rangle
$$

donde $\left|\psi_{i}\left(\mathbf{r}^{\prime}\right)\right\rangle$ son las funciones de onda de una simple partícula perteneciente a las $N$ soluciones de la ecuación de Kohn-Sham:

$$
\hat{\mathcal{H}}_{K S}\left|\psi_{i}\left(\mathbf{r}^{\prime}\right)\right\rangle=\varepsilon_{i}\left|\psi_{i}\left(\mathbf{r}^{\prime}\right)\right\rangle
$$

Las funciones de onda $\left|\psi_{i}\left(\mathbf{r}^{\prime}\right)\right\rangle$ como también las energías $\varepsilon_{i}$ no son las correspondientes a la del electrón, éstas son simplemente funciones matemáticas sin significado físico. Sólo la densidad electrónica total del estado fundamental de éstas funciones ficticias es lo que representa a la densidad electrónica del estado fundamental.

\subsubsection{Funcional de Intercambio-Correlación}

El único termino que se desconoce del $\hat{\mathcal{H}}_{H K}$ es la funcional $\hat{\mathcal{V}}_{x c}$ debido a que no existe una expresión exacta para esta funcional, existiendo sólo aproximaciones para describirla. La aproximación inicialmente desarrollada es la denominada Aproximación de la Densidad Local (LDA, Local Density Approximation), la cual define a $\hat{\mathcal{V}}_{x c}$ como [15].

$$
\hat{\mathcal{V}}_{x c}^{L D A}[n]=\int n(\mathbf{r}) \epsilon_{x c}(n(\mathbf{r})) d \mathbf{r}
$$

donde $\epsilon_{x c}(n(\mathbf{r}))$ es la función de intercambio y correlación para un gas de electrones, que es numéricamente conocida por medio de cálculos de Monte-Carlo [26]. La LDA básicamente considera un gas de electrones cuya densidad varía lentamente en el espacio real. Además, en cada punto del espacio se establece un diferencial de volumen constante donde la energía de intercambio y correlación puede ser aproximada por la energía de intercambio y correlación de un gas homogéneo de electrones, pero conservando la misma densidad electrónica. Algunas de estas funcionales son las propuestas por Vosko-Wilk-Nusair (VWN) [27], Perdew-Zunger (PZ81) [28], Cole-Perdew (CP) [29] y Perdew-Wang (PW92) [30]. 
Otra aproximación utilizada es la Aproximación del Gradiente Generalizado (GGA, Generalized Gradient Approximation) [15,31], en donde se considera a la densidad local $n(\mathbf{r})$ y a su gradiente:

$$
\hat{\mathcal{V}}_{x c}^{G G A}[n]=\int n(\mathbf{r}) \epsilon_{x c}(n(\mathbf{r}),|\nabla n(\mathbf{r})|) d \mathbf{r}
$$

A diferencia del $\hat{\mathcal{V}}_{x c}^{L D A}$ que sólo existe una expresión para el $\epsilon_{x c}$, el $\hat{\mathcal{V}}_{x c}^{G G A}$ puede ser implementado de muchas formas debido a que existen diferentes versiones de $\epsilon_{x c}$ para GGA [32-38], donde algunos de ellos son obtenidos por medio de ajustes de datos experimentales.

Una de las consecuencias de la utilización de estas aproximaciones es que LDA generalmente reproduce parámetros de red 1-3\% más pequeños que el valor experimental, mientras que GGA en muchos casos sobreestima levemente el valor del parámetro de red.

\subsubsection{Funcional de la Densidad de Espín}

En todos los desarrollos previos de la densidad total $n(\mathbf{r})$ no han sido considerados los espines, los cuales son necesarios para describir sistemas magnéticos. Para lo cual se introduce un parámetro adicional a la DFT, relacionado con el momento magnético, $m=n_{\uparrow}(\mathbf{r})-n_{\downarrow}(\mathbf{r})$ con el cual se obtiene un mayor control sobre $m$ debido a su estabilidad numérica, donde $n_{\uparrow}(\mathbf{r})$ y $n_{\downarrow}(\mathbf{r})$ representan las densidades de espín up y down, respectivamente. En este contexto, la densidad electrónica total, $n(\mathbf{r})$, puede ser descrita en función de dos densidades de espín [15].

$$
n(\mathbf{r})=n_{\uparrow}(\mathbf{r})+n_{\downarrow}(\mathbf{r})
$$

Considerando los dos teoremas de Hohenberg-Kohn:

$$
\begin{aligned}
\langle\Psi|\hat{\mathcal{O}}| \Psi\rangle & =O\left[n_{\uparrow}, n_{\downarrow}\right] \\
\left\langle\Psi\left|\hat{E}_{V_{e x t}}\right| \Psi\right\rangle & =E_{V_{e x t}}\left[n_{\uparrow}, n_{\downarrow}\right]
\end{aligned}
$$

Ahora, cada observable es una funcional de la densidad de espines. La densidad de espines del estado fundamental puede ser obtenida minimizando la funcional de energía total del estado fundamental $E_{V_{e x t}}\left[n_{\uparrow}, n_{\downarrow}\right]$. EN este contexto, se ha 
encontrado que la LDA con polarización de espines describe de forma ineficiente a los compuestos magnéticos, reproduciendo erróneamente el estado fundamental magnético, por ejemplo el Fe (BCC) [39-41], mientras que GGA con polarización de espín describe eficientemente el estado fundamental magnético pero mucha veces sobreestima los valores de los momentos magnético.

\subsection{Solución de la Ecuación de Kohn-Sham}

Teniendo en cuenta que el hamiltoniano (1.17) depende de la densidad electrónica a través del término de Hartree y del intercambio y correlación, mientras que la densidad electrónica depende de la función de onda $\left|\psi_{i}\left(\mathbf{r}^{\prime}\right)\right\rangle$. Debido a la complejidad de las ecuaciones de K-S y que no se puede obtener una expresión matemáticamente cerrada para su solución, se debe apelar a solucionarlo en forma numérica. Esto conlleva a un problema de autoconsistencia, donde un procedimiento iterativo es necesario para solucionarlo. Para la primera iteración se considera una densidad de prueba, la cual permite construir el $\hat{\mathcal{H}}_{H K}$ inicial, al solucionar esta ecuación se obtiene un conjunto de $\left|\psi_{i}\left(\mathbf{r}^{\prime}\right)\right\rangle$ y por lo tanto una nueva densidad electrónica $n(\mathbf{r})$. La nueva densidad electrónica permite obtener una nueva $\hat{\mathcal{H}}_{H K}$, este procedimiento se repite hasta obtener la autoconsistencia de la densidad electrónica. Teniendo una densidad electrónica convergida, se pueden obtener los valores de expectación de los observables, la Figura 1.1 muestra un esquema de dicho procedimiento. Por lo tanto, la ecuación de K-S es una herramienta práctica para poder encontrar la solución al problema de muchos cuerpos.

Para encontrar la solución de la ecuación de Kohn-Sham:

$$
\left(-\frac{\hbar^{2}}{2 m_{e}} \nabla_{i}^{2}+\frac{e^{2}}{4 \pi \varepsilon_{0}} \int \frac{n(\mathbf{r})}{\left|\mathbf{r}-\mathbf{r}^{\prime}\right|} d \mathbf{r}^{\prime}+\mathcal{V}_{x c}+\mathcal{V}_{e x t}\right)\left|\psi_{i}(\mathbf{r})\right\rangle=\varepsilon_{i}\left|\psi_{i}(\mathbf{r})\right\rangle
$$

se debe de buscar un conjunto de bases que sean lo suficientemente eficientes y permita describir a las funciones de onda de los átomos. Un importante paso es considerar a $\left|\psi_{i}\left(\mathbf{r}^{\prime}\right)\right\rangle$ como la expansión de un determinado conjunto de bases de- 
nominados $\left\{\left|\phi_{p}^{b}\right\rangle\right\}_{p=1, \cdots, m}$ :

$$
\left|\psi_{i}\right\rangle=\sum_{p=1}^{m} c_{p}^{i}\left|\phi_{p}^{b}\right\rangle
$$

En principio estas bases son infinitas $(P \rightarrow \infty)$, pero en la práctica el conjunto de bases debe ser finita, implicando que una descripción exacta de $\left|\psi_{i}(\mathbf{r})\right\rangle$ es imposible, debido a esto es importante considerar un adecuado límite del conjunto de bases para obtener una buena aproximación de $\left|\psi_{i}(\mathbf{r})\right\rangle$.

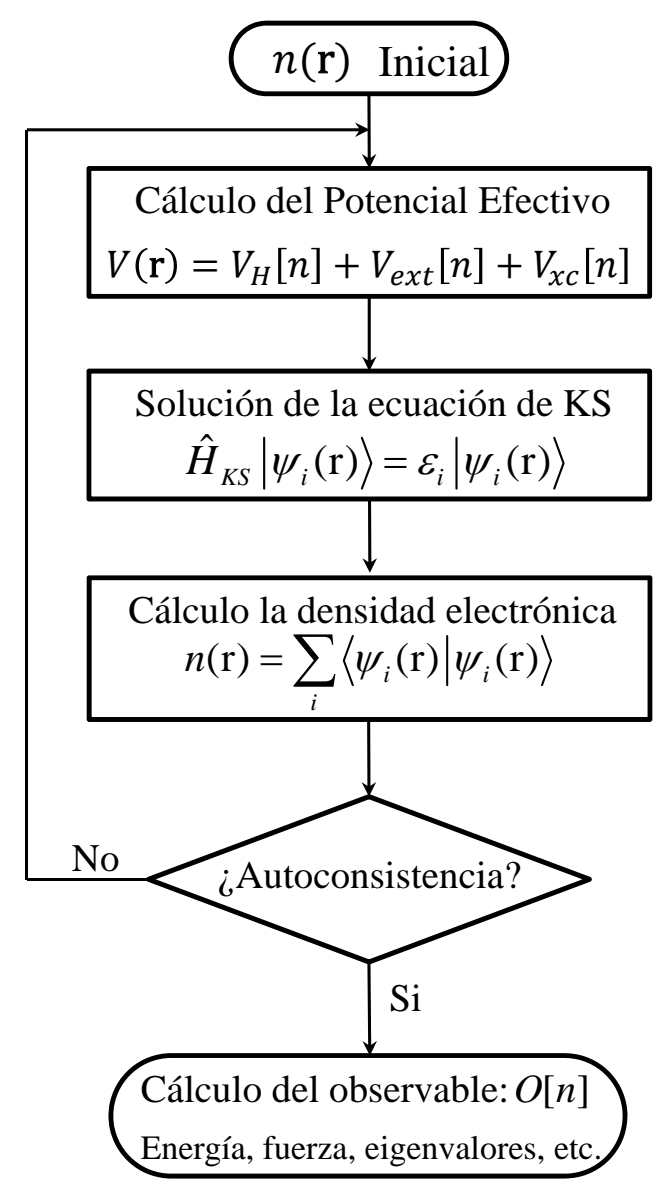

Figura 1.1: Diagrama de flujo del proceso autoconsistente de la Ecuación de Kohn-Sham.

Sustituyendo (1.24) en (1.23), se obtiene un problema de autovalores y autovec- 
tores [42]:

$$
\left[\begin{array}{ccc}
\cdots & \cdots & \cdots \\
\vdots & \left\langle\phi_{j}^{b}|\hat{\mathcal{H}}| \phi_{i}^{b}\right\rangle-\varepsilon_{i}\left\langle\phi_{j}^{b} \mid \phi_{i}^{b}\right\rangle & \vdots \\
\cdots & \cdots & \cdots
\end{array}\right]\left[\begin{array}{c}
c_{1}^{i} \\
\vdots \\
c_{p}^{i}
\end{array}\right]=\left[\begin{array}{c}
0 \\
0 \\
0
\end{array}\right]
$$

Las $P$ autofunciones y autovalores pueden ser encontrados diagonalizando esta matriz, al incrementar el valor de $P$ se incrementará el número de autofunciones y autovalores, obteniéndose una mayor eficiencia de la aproximación. Tanto la eficiencia de la aproximación como el tiempo de cálculo computacional necesario dependen fuertemente con el número de elementos de la base que se tiene, además de la forma de éstas.

\subsubsection{El Método APW}

Históricamente el primer ejemplo importante de una base eficiente fue propuesta por Slate en 1937 [15,43] denominada Augmented Plane Wave (APW), aunque actualmente la base APW es ineficiente, dio origen a otras bases con mayor eficiencia.

El método APW, el espacio es dividido en dos regiones en donde diferentes bases son usadas. La región alrededor del núcleo atómico $(\alpha)$ es descrita por esferas de muffin-tin $(M T)$ no sobrepuestas $\left(S_{M T, \alpha}\right)$ de radio $R_{M T, \alpha}$. Mientras que la región entre las esferas se denomina región intersticial $(I)$ [44], ver Figura 1.2.

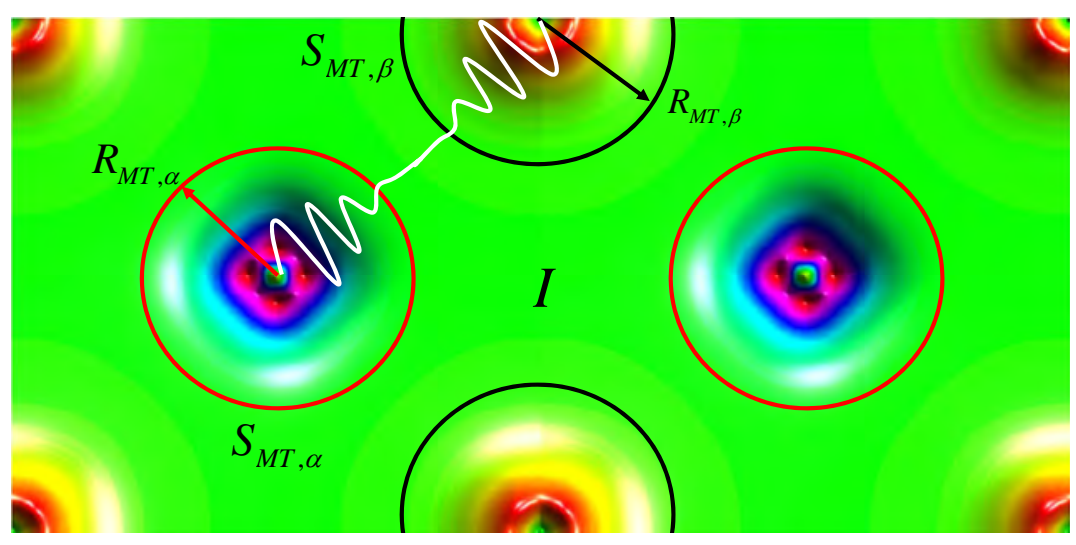

Figura 1.2: División del espacio de un sólido: Esferas de muffin-tin $S_{M T, \alpha}$ y $S_{M T, \beta}$ centradas en los átomos $\alpha$ y $\beta$ y el espacio intersticial $(I)$. 
Este método considera al potencial que rodea al átomo de forma esférica y constante en el espacio interatómico, además que, las funciones de onda cercanas al núcleo son semejantes a la de un átomo aislado, las cuales oscilan fuertemente. Esta elección hace que se requieran muchas ondas planas para describir las funciones de onda, implicando un incremento en el tiempo computacional.

Así, la base APW se define como [15,42,44]:

$$
\phi_{\mathbf{K}}^{\mathbf{k}}(\mathbf{r}, E)= \begin{cases}\frac{1}{\sqrt{\Omega}} e^{i(\mathbf{k}+\mathbf{K}) \cdot \mathbf{r}} & ; \mathbf{r} \in I \\ \sum_{\ell, m} A_{\ell, m}^{\alpha, \mathbf{k}+\mathbf{K}_{\mathcal{U}}} \mathcal{U}_{\ell}^{\alpha}\left(\mathbf{r}_{i}, E\right) Y_{m}^{\ell}\left(\mathbf{r}_{i}\right) & ; \mathbf{r} \in S_{M T, \alpha}\end{cases}
$$

donde $\mathbf{k}$ y $\mathbf{K}$ son vectores de la red recíproca, el primero perteneciente a la primera zona de Brillouin, $\Omega$ es el volumen de la celda unidad en el espacio real, $Y_{\ell}^{m}\left(\mathbf{r}_{i}\right)$ son los armónicos esféricos con índices de momento angular $\ell, m$. Además, $\mathbf{r}_{i}=\mathbf{r}-\mathbf{r}_{\alpha}$, donde $\mathbf{r}_{\alpha}$ es la posición del átomo $\alpha$ en la celda unidad, mientras que $A_{\ell, m}^{\alpha, \mathbf{k}+\mathbf{K}}$ son los coeficientes de expansión y $\mathcal{U}_{\ell}^{\alpha}\left(\mathbf{r}_{i}, E\right)$ es la solución de la ecuación radial de Schrödinger con un potencial esférico promedio $\mathcal{V}(\mathbf{r})$ centrado sobre un átomo:

$$
\left[\frac{d^{2}}{d r^{2}}+\frac{\ell(\ell+1)}{r^{2}}+\mathcal{V}(r)-E\right] r \cdot \mathcal{U}_{\ell}^{\alpha}\left(\mathbf{r}_{i}, E\right)=0
$$

Para lo cual, $\mathcal{U}_{\ell}^{\alpha}\left(\mathbf{r}_{i}, E\right)$ debe de tener continuidad con la correspondiente onda plana en el límite de la esfera de MT, permitiendo determinar los coeficientes $A_{\ell, m}^{\alpha, \mathbf{k}+\mathbf{K}}$.

El método APW tiene un gran inconveniente, debido a que $\mathcal{U}_{\ell}^{\alpha}\left(\mathbf{r}_{i}, E\right)$ depende de la energía $E$ la cual es desconocida. Esto implica que, para describir propiamente una autofunción $\left|\psi_{i}(\mathbf{r})\right\rangle$ de la ecuación de Kohn-Sham (1.21), es necesario conocer el correspondiente autovalor $\varepsilon_{i}$, el cual será usado para obtener $E$. Una solución general a este problema consiste en algunas mejorías de la bas dentro de las esferas de MT, de tal forma de eliminar la dependencia con la energía. 


\subsubsection{El Método LAPW}

Una solución para el problema presente en el método APW radica en considerar que en cierta región de energías las APW son independientes ellas. Este método se denomina Linearized Augmented Plane Wave (LAPW) [15, 42, 45, 46] el cual divide al espacio de forma semejante al método APW. Para la región intersticial la base LAPW es la misma que APW, pero en la esfera de muffin-tin varía y se expresa como:

$$
\phi_{\mathbf{K}}^{\mathbf{k}}(\mathbf{r}, E)= \begin{cases}\frac{1}{\sqrt{\Omega}} e^{i(\mathbf{k}+\mathbf{K}) \cdot \mathbf{r}} & ; \mathbf{r} \in I \\ \sum_{\ell, m}\left[A_{\ell, m}^{\alpha, \mathbf{k}+\mathbf{K}} \mathcal{U}_{\ell}^{\alpha}\left(\mathbf{r}_{i}, E\right)+B_{\ell, m}^{\alpha, \mathbf{k}+\mathbf{K}} \dot{\mathcal{U}}_{\ell}^{\alpha}\left(\mathbf{r}_{i}, E\right)\right] Y_{m}^{\ell}\left(\mathbf{r}_{i}\right) & ; \mathbf{r} \in S_{M T, \alpha}\end{cases}
$$

De (1.28) se puede observar que la región dentro de la esfera de MT se describe por una combinación lineal de la original función $\mathcal{U}_{\ell}^{\alpha}$ y su derivada en energía $\dot{\mathcal{U}}_{\ell}^{\alpha} \equiv$ $\frac{\partial \mathcal{U}_{\ell}^{\alpha}\left(\mathbf{r}_{i}, E\right)}{\partial E}$, evaluada en $E_{0}$, el cual se puede interpretar como una expansión de Taylor de primer orden alrededor de cierta energía fija $E_{0}$.

$$
\mathcal{U}_{\ell}^{\alpha}\left(\mathbf{r}_{i}, E\right)=\mathcal{U}_{\ell}^{\alpha}\left(\mathbf{r}_{i}, E_{0}\right)+\left(E-E_{0}\right) \dot{\mathcal{U}}_{\ell}^{\alpha}\left(\mathbf{r}_{i}, E_{0}\right)+\mathcal{O}\left(E-E_{0}\right)^{2}
$$

Si la energía $E_{0}$ difiere levemente de la verdadera energía de banda $E$, una combinación lineal reproducirá la función radial APW de la energía de banda. Además, los coeficientes $A_{\ell, m}^{\alpha, \mathbf{k}+\mathbf{K}}$ y $B_{\ell, m}^{\alpha, \mathbf{k}+\mathbf{K}}$ pueden ser determinados bajo las condiciones de continuidad de las funciones de onda en los límites de la esfera de MT. Estos coeficientes no pueden ser determinados por medio de una expansión de Taylor porque este proceso introduciría una dependencia con la energía, que nos guiaría al mismo problema del APW.

Generalizando este criterio para todos los valores de $\ell$ del átomo $\alpha$, donde $E_{\ell}^{\alpha}$ es normalmente elegido en el centro de la correspondiente banda de carácter $\ell$, se obtiene la base $[15,42,46]$ :

$$
\phi_{\mathbf{K}}^{\mathbf{k}}(\mathbf{r}, E)= \begin{cases}\frac{1}{\sqrt{\Omega}} e^{i(\mathbf{k}+\mathbf{K}) \cdot \mathbf{r}} & ; \mathbf{r} \in I \\ \sum_{\ell, m}\left[A_{\ell, m}^{\alpha, \mathbf{k}+\mathbf{K}} \mathcal{U}_{\ell}^{\alpha}\left(\mathbf{r}_{i}, E_{\ell}^{\alpha}\right)+B_{\ell, m}^{\alpha, \mathbf{k}+\mathbf{K}} \dot{\mathcal{U}}_{\ell}^{\alpha}\left(\mathbf{r}_{i}, E_{\ell}^{\alpha}\right)\right] Y_{m}^{\ell}\left(\mathbf{r}_{i}\right) & ; \mathbf{r} \in S_{M T, \alpha}\end{cases}
$$


Si $E_{\ell}^{\alpha}$ es cuidadosamente elegido para cada valor del momento angular, una simple diagonalización producirá un eficiente conjunto de energías de bandas para cada punto $\mathrm{k}$ de la red recíproca. Las bases LAPW definidas en (1.30), posee aún dos parámetros que deben ser optimizados, el primero es $\ell_{\max }$ que controla el tamaño de la base LAPW, mientras que el segundo parámetro es la onda plana de corte, $\mathbf{K}_{\max }$, el cual está relacionada con la energía de corte y determina el tamaño del conjunto de la base, es decir, sólo las funciones que pertenezcan a la base con un $\mathbf{K}$ que satisfaga la condición $\left|\mathbf{k} \leq K_{\max }\right|$ serán incluidas en el conjunto de los elementos de la base $[15,42]$. Como consecuencia, $\ell_{\max }$ y $K_{\max }$ controlan la eficiencia y calidad del cálculo.

Además, los parámetros $\ell_{\max }$ y $K_{\max }$ no son completamente independientes debido a que en las condiciones de contorno impuestas estos dos parámetros deben de coincidir. Dado que para un determinado $\ell_{\max }$, la función $Y_{m}^{\ell_{\max }}$ tendrá a lo sumo $2 \ell_{\max }$ nodos a lo largo de una circunferencia alrededor de la esfera de MT, es decir sobre un circulo de longitud $2 \pi R_{M T}$. Esto corresponde a $\frac{\ell_{\max }}{\pi R_{M T}}$ nodos por unidad de longitud $[15,42]$. Por otro lado, $\mathbf{K}_{\max }$ describe una onda plana con $\frac{\mathbf{K}_{\max }}{\pi}$ nodos por unidad de longitud. Igualando estas cantidades, se obtiene:

$$
R_{M T} \times K_{\max }=\ell_{\max }
$$

Esto reduce la optimización de un sólo parámetro, de tal forma que si se conoce el valor de $\mathbf{K}_{\max }$ entonces será posible obtener el valor de $\ell_{\max }$. 


\section{Capítulo 2}

\section{Antecedentes y Metodología}

\subsection{Antecedentes Experimentales y Teóricos}

$\mathrm{El} \gamma^{\prime}-\mathrm{Fe}_{4} \mathrm{~N}$ es un compuesto que inicialmente llamó la atención por sus posibles aplicaciones en la catálisis para la producción de amonio y por su importancia en el tratamiento de superficies de acero con amonio [10]. Usando difracción de Rayos $\mathrm{X}$ en muestras de polvo de $\gamma^{\prime}-\mathrm{Fe}_{4} \mathrm{~N}$, se determinó que este compuesto posee una estructura cristalina cúbica con grupo espacial $\operatorname{P} m \overline{3} m[47,48]$, esto fue confirmado después en muestras de cristales $[49,50]$, determinándose así que en el $\gamma^{\prime}-\mathrm{Fe}_{4} \mathrm{~N}$ se pueden distinguir dos tipos de átomos de Fe, el FeI que se encuentra en la esquina de la celda cúbica, posición cartesiana $(0,0,0)$, y los FeII que se encuentran en la esquina de la celda cúbica, posiciones cartesianas $\left(\frac{1}{2}, \frac{1}{2}, 0\right),\left(\frac{1}{2}, 0, \frac{1}{2}\right)$ y $\left(0, \frac{1}{2}, \frac{1}{2}\right)$, mientras que el átomo de $\mathrm{N}$ se ubica en el centro de la celda cristalina, posición cartesiana $\left(\frac{1}{2}, \frac{1}{2}, \frac{1}{2}\right)$. Estos sitios cristalográficos también se denominan sitios $1 a, 3 c$ y $1 b$, respectivamente, en la notación de Wyckoff (Figura 2.1). 


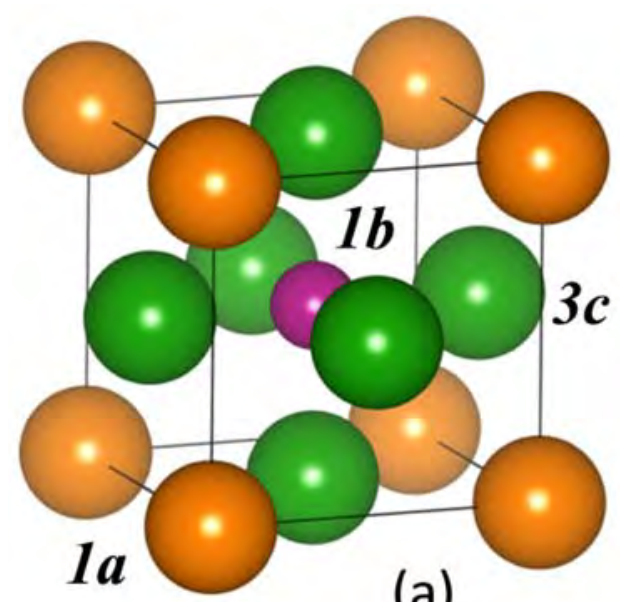

(a)

Figura 2.1: Estructura Cristalina del $\gamma^{\prime}-\mathrm{Fe}_{4} \mathrm{~N}$.

Posteriormente fueron caracterizadas las propiedades magnéticas e hiperfinas del $\gamma^{\prime}-\mathrm{Fe}_{4} \mathrm{~N}$, mostrando una elevada magnetización, además de un alto momento magnético por átomo de Fe, semejante al del $\alpha$-Fe $\left(2.2 \mu_{B}\right)$ [51]. Medidas de difracción de neutrones mostraron que el $\gamma^{\prime}-\mathrm{Fe}_{4} \mathrm{~N}$ es un ferromagneto con momento magnético de $3.0 \mu_{B}$ y $2.0 \mu_{B}$ para el FeI y el FeII, respectivamente [52]. Estos resultados fueron corroborados por medio de la espectroscopia Mössbauer [53-55]. Además, posee una alta magnetización de saturación de 190 emu/gr [56], semejante al del $\alpha-\mathrm{Fe}$ (218 emu/gr), una baja coercitividad, $H_{c}=5.8$ Oe [57] y con dirección principal de magnetización [001] [58-60]. Todas estas propiedades magnéticas del $\gamma^{\prime}-\mathrm{Fe}_{4} \mathrm{~N}$ hizo a este compuesto un buen candidato para aplicaciones en dispositivos para el grabado magnético de alta densidad [11-14]. Otras de las posibles aplicaciones del $\gamma^{\prime}-\mathrm{Fe}_{4} \mathrm{~N}$ es el recubrimiento de productos de acero debido a que posee una alta dureza y resistencia a la corrosión [61,62], siendo este un material dúctil y tolerante a daños debido a su alto valor del módulo de bulk [63,64].

En las últimas décadas, muchos trabajos experimentales han reportado el crecimiento epitaxial de películas de $\gamma^{\prime}-\mathrm{Fe}_{4} \mathrm{~N}$ sobre distintos substratos, en diferentes condiciones y con diversos métodos de crecimiento de dichas películas. Además se han realizado medidas en polvos y nanocompuestos de $\gamma^{\prime}-\mathrm{Fe}_{4} \mathrm{~N}$, dando origen a un gran abanico de posibles valores del parámetro de red $(a)$, magnetización y 
parámetros hiperfinos de dicho compuesto, difiriendo muchos de ellos de las medidas realizadas en cristales. Estas diferencias son atribuidas a efectos de substrato, efectos superficiales para el caso de películas o al tamaño de grano en el caso de polvos. En la Tabla 2.1, se muestra una recopilación bibliográfica de los diferentes valores experimentales del parámetro de red $(a)$ obtenidas de diferentes tipos de muestras. El valor del parámetro de red del $\gamma^{\prime}-\mathrm{Fe}_{4} \mathrm{~N}$ ampliamente aceptado es la obtenida por H. Jacob et. al [50]. Mientras que en la Tabla 2.2 se ha recopilado los valores experimentales de la magnetización del $\gamma^{\prime}-\mathrm{Fe}_{4} \mathrm{~N}$ y los parámetros hiperfinos como el campo hiperfino $\left(\mathrm{B}_{h f}\right)$, corrimiento isomérico $(\delta)$ y el desdoblamiento cuadrupolar $(\varepsilon)$ de los átomos de Fe del $\gamma^{\prime}-\mathrm{Fe}_{4} \mathrm{~N}$. 
Tabla 2.1: Valores experimentales del parámetro (a) de red del $\gamma^{\prime}-\mathrm{Fe}_{4} \mathrm{~N}$ para diferentes tipos de muestra, obtenidos de la bibliografía.

\begin{tabular}{|c|c|c|c|c|c|}
\hline$a(\AA)$ & $\mathrm{T}(\mathrm{K})$ & Tipo de muestra & Substrato & Método & Ref. \\
\hline 3.7970 & RT & Cristales & - & Flujo $\mathrm{NH}_{3} / \mathrm{H}_{2}$ & [53] \\
\hline 3.7900 & RT & Cristales & - & Flujo $\mathrm{NH}_{3} / \mathrm{H}_{2}$ & [50] \\
\hline 3.7950 & $\mathrm{RT}$ & Cristales & - & Flujo $\mathrm{NH}_{3} / \mathrm{H}_{2}$ & [65] \\
\hline 3.7800 & RT & Nanocristales & - & Flujo $\mathrm{NH}_{3} / \mathrm{H}_{2}$ & [66] \\
\hline 3.7990 & RT & Nanopartículas & - & Flujo $\mathrm{NH}_{3} / \mathrm{H}_{2}$ & [67] \\
\hline 3.7870 & $\mathrm{RT}$ & Nanopartículas & - & Flujo $\mathrm{NH}_{3} / \mathrm{H}_{2}$ & [68] \\
\hline 3.7900 & RT & Nanodendritas & - & Flujo $\mathrm{NH}_{3} / \mathrm{H}_{2}$ & [69] \\
\hline 3.7900 & RT & Polvos & - & Flujo $\mathrm{NH}_{3} / \mathrm{H}_{2}$ & [49] \\
\hline 3.7940 & RT & Polvos & - & Flujo $\mathrm{NH}_{3} / \mathrm{H}_{2}$ & [70] \\
\hline 3.7950 & RT & Películas delgadas & $\mathrm{MgO}(100)$ & $\mathrm{MBE}$ & [71] \\
\hline 3.7950 & RT & Películas delgadas & $\mathrm{MgO}(100)$ & MBE & [72] \\
\hline 3.7950 & $\mathrm{RT}$ & Películas delgadas & Corning glass & DC-MS & [73] \\
\hline 3.7950 & $\mathrm{RT}$ & Películas delgadas (36nm) & $\mathrm{MgO}(001)$ & MBE & [59] \\
\hline 3.7950 & RT & Películas delgadas (90nm) & $\mathrm{MgO}(001)$ & MBE & [74] \\
\hline 3.7900 & 280 & Películas delgadas (80nm) & $\mathrm{MgO}(001)$ & MBE & [75] \\
\hline 3.7950 & RT & Películas delgadas (2ML) & $\mathrm{Cu}(001)$ & MBE & [76] \\
\hline 3.7910 & RT & Películas delgadas $(8,12 \mathrm{~nm})$ & $\mathrm{LaAlO}_{3}(001), \mathrm{SrTiO}_{3}(001)$ & MBE & [77] \\
\hline 3.7950 & 300 & Películas delgadas (10nm) & $\mathrm{LaAlO}_{3}(001), \mathrm{MgO}(001)$ & MBE & [78] \\
\hline 3.7950 & RT & Películas thick (16nm) & $\mathrm{MgO}(001)$ & MBE & [79] \\
\hline 3.7950 & RT & Películas (200nm) & Corning glass & DC-MS & [80] \\
\hline 3.7950 & RT & Películas (270ML) & $\mathrm{Cu}(100)$ & MBE & [81] \\
\hline 3.7900 & $\mathrm{RT}$ & Películas (200ML) & $\mathrm{Cu}(100)$ & MBE & [82] \\
\hline 3.7950 & RT & Películas (45nm) & $\mathrm{TiN}(001) / \mathrm{Si}(001)$ & DC-MS & [83] \\
\hline 3.7890 & 475 & Películas (200nm) & $\operatorname{Si}(100)$ & PLD & [84] \\
\hline 3.7950 & RT & Película Granular (80nm) & $\mathrm{NaCl} / \mathrm{Si}(100)$ & Sputtering & [85] \\
\hline
\end{tabular}

Consecuentemente, surgió un gran interés en los compuestos ternarios $X \mathrm{Fe}_{3} \mathrm{~N}$, donde $X$ sustituye a un átomo de $\mathrm{Fe}(\mathrm{Fe} I$ o $\mathrm{Fe} I I)$ y puede ser un metal de transición de la serie $3 d(\mathrm{Mn}, \mathrm{Ni}, \mathrm{Cu}$ o $\mathrm{Zn}$ ), de la serie $4 d(\mathrm{Ru}, \mathrm{Rh}, \mathrm{Pd}$ o $\mathrm{Ag})$, e inclusive un metal del bloque $p$ (Al, Ga, In o Sn). Dicho interés surgió con la finalidad de encontrar un compuesto ternario con propiedades semejantes al $\gamma^{\prime}-\mathrm{Fe}_{4} \mathrm{~N}$. Existiendo muy poca información experimental sobre estos sistemas ternarios, debido a dificultades en 
Tabla 2.2: Valores experimentales del momento magnético $\left(\mathrm{M}_{f u}\right)$ del $\gamma^{\prime}-\mathrm{Fe}_{4} \mathrm{~N}$, momento magnético por átomos $(m)$, campo hiperfino $\left(\mathrm{B}_{h f}\right)$, corrimiento isomérico $(\delta)$ y desdoblamiento cuadrupolar ( $\varepsilon$ ) de los átomos de Fe del $\gamma^{\prime}-\mathrm{Fe}_{4} \mathrm{~N}$.

\begin{tabular}{|c|c|c|c|c|c|c|c|c|c|c|c|}
\hline \multirow{2}{*}{$\mathrm{M}_{f u}\left(\mu_{B}\right)$} & \multicolumn{2}{|c|}{$\mathrm{m}\left(\mu_{B}\right)$} & \multicolumn{2}{|c|}{$\mathrm{B}_{h f}(\mathrm{~T})$} & \multicolumn{2}{|c|}{$\delta(m m / s)$} & \multicolumn{3}{|c|}{$\varepsilon(m m / s)$} & \multirow{2}{*}{$\mathrm{T}(\mathrm{K})$} & \multirow{2}{*}{ Ref. } \\
\hline & $\mathrm{Fe} I$ & FeII & $\mathrm{Fe} I$ & FeII & $\mathrm{Fe} I$ & FeII & $\mathrm{Fe} I$ & FeIIa & FeIIb & & \\
\hline 8.71 & - & - & -36.9 & -23.5 & - & - & - & - & - & 1.3 & [86] \\
\hline 9.39 & - & - & - & - & - & - & - & - & - & 2.0 & [87] \\
\hline 8.90 & - & - & - & - & - & - & - & - & - & 4.2 & [55] \\
\hline- & - & - & -36.9 & -23.6 & 0.38 & 0.42 & - & 0.27 & -0.54 & 4.2 & [88] \\
\hline- & - & - & -36.9 & -23.4 & - & - & - & - & - & 4.2 & [89] \\
\hline- & - & - & -37.0 & -23.7 & 0.26 & 0.32 & - & 0.22 & -0.47 & 4.2 & [90] \\
\hline- & - & - & -37.0 & -23.6 & 0.39 & 0.46 & - & 0.24 & -0.52 & 5.0 & [91] \\
\hline 9.22 & - & - & - & - & - & - & - & - & - & 5.0 & [92] \\
\hline 7.31 & - & - & -36.8 & -23.8 & 0.38 & 0.45 & 0.03 & 0.27 & -0.17 & 78 & [93] \\
\hline 8.50 & - & - & - & - & - & - & - & - & - & 80 & [94] \\
\hline 8.63 & - & - & - & - & - & - & - & - & - & 280 & [75] \\
\hline 9.04 & 3.01 & 2.01 & - & - & - & - & - & - & - & $\mathrm{RT}$ & [52] \\
\hline 9.89 & - & - & - & - & - & - & - & - & - & RT & [53] \\
\hline- & - & - & -34.1 & -21.6 & - & - & - & - & - & RT & [95] \\
\hline- & - & - & -34.1 & -21.7 & 0.25 & 0.31 & 0.00 & 0.16 & -0.24 & RT & [96] \\
\hline- & - & - & -33.1 & -21.8 & 0.14 & 0.22 & - & - & - & RT & [97] \\
\hline- & - & - & -34.0 & -21.2 & 0.16 & 0.25 & - & - & - & RT & [98] \\
\hline- & - & - & -34.0 & -21.6 & 0.20 & 0.27 & 0.01 & 0.10 & -0.21 & RT & [70] \\
\hline- & - & - & -33.6 & -20.1 & 0.16 & 0.26 & 0.07 & 0.15 & -0.16 & RT & [99] \\
\hline- & - & - & -34.4 & -21.8 & 0.23 & 0.30 & 0.01 & 0.19 & -0.37 & RT & [66] \\
\hline 7.83 & - & - & - & - & - & - & - & - & - & RT & [71] \\
\hline 7.74 & - & - & - & - & - & - & - & - & - & RT & [72] \\
\hline- & - & - & -34.4 & -21.8 & 0.23 & 0.30 & 0.01 & 0.19 & -0.37 & RT & [100] \\
\hline 8.00 & - & - & - & - & - & - & - & - & - & RT & [80] \\
\hline- & - & - & -34.1 & -21.7 & 0.22 & 0.29 & 0.00 & 0.24 & -0.45 & RT & [79] \\
\hline 7.89 & - & - & -34.4 & -21.8 & - & - & 0.01 & 0.26 & - & RT & [68] \\
\hline 8.00 & - & - & - & - & - & - & - & - & - & RT & [73] \\
\hline- & - & - & -34.1 & -21.7 & 0.22 & 0.29 & 0.00 & 0.24 & -0.45 & RT & [59] \\
\hline- & - & - & -33.3 & -20.8 & - & - & 0.01 & 0.17 & -0.40 & RT & [81] \\
\hline 9.01 & 2.98 & 2.01 & - & - & - & - & - & - & - & RT & [82] \\
\hline 7.69 & - & - & - & - & - & - & - & - & - & RT & [101] \\
\hline- & - & - & -34.4 & -21.8 & 0.25 & 0.31 & 0.01 & 0.19 & -0.40 & RT & [102] \\
\hline- & - & - & -35.1 & -23.0 & 0.23 & 0.30 & 0.01 & - & -0.47 & RT & [102] \\
\hline- & - & - & -34.4 & -21.9 & 0.23 & - & 0.01 & - & -0.40 & RT & [102] \\
\hline 9.55 & - & - & - & - & - & - & - & - & - & RT & [83] \\
\hline 7.95 & - & - & - & - & - & - & - & - & - & RT & [84] \\
\hline 9.90 & 2.44 & - & - & - & - & - & - & - & - & RT & [78] \\
\hline
\end{tabular}


la síntesis de dichos compuestos o a la ausencia de dichas fases cristalinas. En la Tabla 2.3, se muestran los valores experimentales obtenidos de la bibliografía del parámetro de red y el método de síntesis, mientras que en la Tabla 2.4 se muestran las propiedades magnéticas e hiperfinas de dichos compuestos.

Tabla 2.3: Valores experimentales del parámetro de red (a) de los compuestos ternarios $\mathrm{XFe}_{3} \mathrm{~N}$ existentes en la bibliografía.

\begin{tabular}{ccclllc}
\hline $\mathrm{X}$ & $a(\AA)$ & $\mathrm{T}(\mathrm{K})$ & Tipo de muestra & Substrato & Método & Ref. \\
\hline $\mathrm{Mn}$ & 3.7979 & RT & Nanocompuesto & - & Flujo $\mathrm{NH}_{3} / \mathrm{H}_{2}$ & {$[12]$} \\
\hline $\mathrm{Ni}$ & 3.7840 & RT & Nanocompuesto & - & Flujo $\mathrm{NH}_{3} / \mathrm{H}_{2}$ & {$[103]$} \\
& 3.7860 & RT & Nanocompuesto & - & Flujo $\mathrm{NH}_{3} / \mathrm{H}_{2}$ & {$[53]$} \\
& 3.7810 & RT & Polvo & - & Flujo $\mathrm{NH}_{3} / \mathrm{H}_{2}$ & {$[49]$} \\
& 3.7875 & RT & Nanocompuesto & - & Flujo $\mathrm{NH}_{3} / \mathrm{H}_{2}$ & {$[104]$} \\
& 3.7877 & 78 & Polvo & - & Flujo $\mathrm{NH}_{3} / \mathrm{H}_{2}$ & {$[105]$} \\
& 3.7683 & RT & Nanocristales & - & Flujo $\mathrm{NH}_{3} / \mathrm{H}_{2}$ & {$[106]$} \\
& 3.7877 & RT & Nanocompuesto & - & Flujo $\mathrm{NH}_{3} / \mathrm{H}_{2}$ & {$[107]$} \\
& 3.7900 & RT & Cristales & - & Flujo $\mathrm{NH}_{3} / \mathrm{H}_{2}$ & {$[108]$} \\
& 3.7870 & RT & Películas delgadas $(200 \mathrm{~nm})$ & $\mathrm{Si}(111)$ & MBE & {$[109]$} \\
& 3.7830 & RT & Películas delgadas $(55 \mathrm{~nm})$ & $\mathrm{Si}(100)$ & DC-MS & {$[110]$} \\
\hline $\mathrm{Cu}$ & 3.7890 & RT & Polvos & - & Flujo $\mathrm{NH}_{3} / \mathrm{H}_{2}$ & {$[111]$} \\
& 3.8304 & RT & Películas delgada $(150 \mathrm{~nm})$ & $\mathrm{Si}(111)$ & MBE & {$[112]$} \\
\hline $\mathrm{Zn}$ & 3.8003 & RT & Polvo & - & Flujo $\mathrm{NH}_{3} / \mathrm{H}_{2}$ & {$[113]$} \\
& 3.7990 & RT & Películas thick (1400nm) & Si(100) & Sputtering & {$[114]$} \\
\hline $\mathrm{Rh}$ & 3.8337 & RT & Polvos & - & Flujo $\mathrm{NH}_{3} / \mathrm{H}_{2}$ & {$[115]$} \\
& 3.8292 & RT & Polvos & - & Flujo $\mathrm{NH}_{3} / \mathrm{H}_{2}$ & {$[116]$} \\
\hline $\mathrm{Pd}$ & 3.8590 & RT & Polvos & Flujo $\mathrm{NH}_{3} / \mathrm{H}_{2}$ & {$[117]$} \\
& 3.8500 & RT & Películas delgadas $(1400 \mu \mathrm{m})$ & $\mathrm{Si}(100)$ & Sputtering & {$[118]$} \\
\hline
\end{tabular}


Tabla 2.4: Valores experimentales obtenidos de la bibliografía del momento magnético total $\left(M_{f u}\right)$ de los compuestos ternarios $X \mathrm{Fe}_{3} \mathrm{~N}$, momento magnético por átomo $(m)$ junto con el campo hiperfino $\left(B_{h f}\right)$, corrimiento isomérico $(\delta)$ y desdoblamiento cuadrupolar $(\varepsilon)$ de los átomos de Fe.

\begin{tabular}{|c|c|c|c|c|c|c|c|c|c|}
\hline \multirow{2}{*}{$X$} & \multirow{2}{*}{$\mathrm{M}_{f u}\left(\mu_{B}\right)$} & \multicolumn{2}{|c|}{$\mathrm{m}\left(\mu_{B}\right)$} & \multirow{2}{*}{$\begin{array}{c}\mathrm{B}_{h f}(\mathrm{~T}) \\
\text { FeII }\end{array}$} & \multirow{2}{*}{$\begin{array}{c}\delta(\mathrm{mm} / \mathrm{s}) \\
\text { FeII }\end{array}$} & \multicolumn{2}{|c|}{$\varepsilon(m m / s)$} & \multirow{2}{*}{$\mathrm{T}(\mathrm{K})$} & \multirow{2}{*}{ Ref. } \\
\hline & & $X$ & FeII & & & Fella & FeIIb & & \\
\hline $\mathrm{Mn}$ & - & - & - & 21.3 & 0.33 & 0.12 & - & RT & [12] \\
\hline \multirow[t]{11}{*}{$\mathrm{Ni}$} & 6.90 & - & - & - & - & - & - & RT & [53] \\
\hline & 5.00 & - & - & - & - & - & - & RT & [57] \\
\hline & 5.00 & - & - & - & - & - & - & $\mathrm{RT}$ & [119] \\
\hline & 6.00 & 1.59 & 0.67 & 19.0 & 0.31 & - & - & $\mathrm{RT}$ & [104] \\
\hline & - & - & - & 22.0 & - & - & - & RT & [120] \\
\hline & 6.71 & 2.20 & 0.40 & - & - & - & - & RT & [105] \\
\hline & - & - & - & 17.1 & - & 0.16 & - & RT & [121] \\
\hline & - & - & - & 20.1 & 0.30 & - & - & RT & [107] \\
\hline & - & - & - & 23.4 & 0.33 & 1.03 & - & $\mathrm{RT}$ & [109] \\
\hline & - & - & - & 22.5 & 0.34 & 0.29 & - & $\mathrm{RT}$ & [122] \\
\hline & 5.50 & 2.20 & 0.60 & - & - & - & - & $\mathrm{RT}$ & [110] \\
\hline \multirow[t]{2}{*}{$\mathrm{Cu}$} & - & - & - & 21.7 & 0.33 & - & - & RT & [111] \\
\hline & - & - & - & 30.2 & 0.22 & 1.19 & - & $\mathrm{RT}$ & [112] \\
\hline $\mathrm{Zn}$ & 4.65 & 1.55 & - & 16.1 & 0.31 & 0.08 & - & RT & [113] \\
\hline $\mathrm{Rh}$ & 8.30 & - & - & - & - & - & - & RT & [117] \\
\hline
\end{tabular}


Desde el punto de vista teórico, usando la Teoría de la Funcional Densidad (DFT), tambien se ha estudiado ampliamente las propiedades estructurales, magnéticas e hiperfinas del $\gamma^{\prime}-\mathrm{Fe}_{4} \mathrm{~N}$, aplicando diferentes métodos para solucionar las ecuaciones de Kohn-Sham. En [129] se encuentra una extensa recopilación bibliográfica de los cálculos teóricos realizados para el compuesto $\gamma^{\prime}-\mathrm{Fe}_{4} \mathrm{~N}$. Además, diferentes autores usando distintos métodos autoconsistentes han abordado el estudio individual de los compuestos ternarios $X \mathrm{Fe}_{3} \mathrm{~N}$, donde siempre han considerado que el átomo $X$ sustituye al átomo FeI, es decir ocupa el sitio 1a de Wyckoff, prediciendo y/o caracterizando así diferentes propiedades de dichos compuestos, pero nunca se ha realizado un estudio sistemático de las propiedades físicas de dichos compuestos usando el método FP-LAPW analizando las posibilidades que el átomo $X$ pueda sustituir a un átomo FeI o FeII. En la Tabla 2.5 se muestra la recopilación bibliográfica de los datos teóricos de las propiedades estructurales, mientras en en la Tabla 2.6 se muestra los valores de las propiedades magnéticas e hiperfinas. 
Tabla 2.5: Valores teóricos recopilados de la bibliografía del parámetro de red (a) y el módulo de bulk (B) de los compuestos ternarios $\mathrm{XFe}_{3} \mathrm{~N}$ con estructura $P m \overline{3} m$.

\begin{tabular}{|c|c|c|c|c|c|c|c|c|c|}
\hline$X$ & $a(\AA)$ & B (GPa) & Método & Ref. & $X$ & $a(\AA)$ & $\mathrm{B}(\mathrm{GPa})$ & Método & Ref. \\
\hline $\mathrm{Ti}$ & 3.7810 & 596.0 & LMTO & [123] & $\mathrm{Cu}$ & 3.8440 & - & LMTO & [111] \\
\hline $\mathrm{V}$ & 3.7300 & 490.0 & LMTO & [124] & $\mathrm{Zn}$ & 3.8304 & - & LMTO & [113] \\
\hline $\mathrm{Cr}$ & 3.7621 & 267.0 & LMTO & [123] & & 3.7650 & 207.0 & PAW-PW & [114] \\
\hline \multirow[t]{4}{*}{ Mn } & 3.7982 & - & LMTO & [125] & $\mathrm{Ru}$ & 3.8500 & - & PAW-PW & [137] \\
\hline & 3.6542 & 266.0 & LMTO & [126] & & 3.7995 & - & FP-LAPW & [142] \\
\hline & 3.7995 & - & FP-LAPW & [127] & & 3.7920 & 198.0 & PP-PW & [143] \\
\hline & 3.7926 & - & LCAO & [128] & & 3.8164 & 195.0 & ASW & [144] \\
\hline \multirow[t]{7}{*}{$\mathrm{Fe}$} & 3.8059 & 191.1 & FP-LAPW /CVM & [129] & $\mathrm{Rh}$ & 3.8700 & - & PP-PW & [115] \\
\hline & 3.7946 & 191.8 & PAW-PW & [130] & & 3.8700 & - & PP-PW & [137] \\
\hline & 3.7943 & 200.0 & FP-LAPW & [131] & & 3.8260 & 213.0 & PP-PW & [135] \\
\hline & 3.7942 & - & FP-LAPW & [132] & & 3.8700 & 187.0 & PP-PW & [116] \\
\hline & 3.7950 & 194.1 & PP-PW & {$[64]$} & $\mathrm{Pd}$ & 3.8800 & - & PP-PW & [137] \\
\hline & - & 166.0 & PAW-PW & [133] & & 3.8704 & - & ASW & [138] \\
\hline & - & 196.0 & PAW-PW & [134] & & 3.8690 & 188.0 & PP-PW & [117] \\
\hline \multirow[t]{4}{*}{ Co } & 3.8857 & 111.0 & LMTO & [135] & & 3.8483 & - & LMTO & [125] \\
\hline & 3.7900 & - & USPP-PW & [136] & & 3.8416 & 444.0 & LMTO & [127] \\
\hline & 3.7550 & 203.0 & PP-PW & [123] & & 3.8610 & 188.0 & PP-PW & [118] \\
\hline & 3.7510 & 204.0 & PP-PW & [137] & $\mathrm{Ag}$ & 3.7824 & - & LMTO & [145] \\
\hline \multirow[t]{7}{*}{$\mathrm{Ni}$} & 3.7820 & - & ASW & [108] & & & & & \\
\hline & 3.7799 & - & ASW & [138] & & & & & \\
\hline & 3.6852 & - & LMTO & [120] & & & & & \\
\hline & 3.8000 & - & USPP-PW & [139] & & & & & \\
\hline & 3.7650 & 190.0 & PAW-PW & [140] & & & & & \\
\hline & 3.7680 & - & MS-X $\alpha$ & [141] & & & & & \\
\hline & 3.7550 & 203.7 & PP-PW & [137] & & & & & \\
\hline
\end{tabular}


Tabla 2.6: Valores teóricos del momento magnético por fórmula unidad $\left(\mathrm{M}_{f u}\right)$, por átomo $(m)$ y parámetros hiperfinos del átomo de Fe de los compuestos $\mathrm{XFe}_{3} \mathrm{~N}$ con estructura cristalina $P m \overline{3} m$.

\begin{tabular}{|c|c|c|c|c|c|c|c|}
\hline \multirow{2}{*}{$X$} & \multirow{2}{*}{$\mathrm{M}_{f u}\left(\mu_{B}\right)$} & \multicolumn{2}{|c|}{$\mathrm{m}\left(\mu_{B}\right)$} & \multirow{2}{*}{$\begin{array}{c}\mathrm{B}_{h f}(\mathrm{~T}) \\
\text { FeII }\end{array}$} & \multirow{2}{*}{$\begin{array}{c}\delta(\mathrm{mm} / \mathrm{s}) \\
\text { FeII }\end{array}$} & \multirow{2}{*}{$\begin{array}{c}\varepsilon(m m / s) \\
\text { FeII }\end{array}$} & \multirow{2}{*}{ Ref. } \\
\hline & & $X$ & FeII & & & & \\
\hline $\mathrm{Ti}$ & 0.00 & 0.00 & 0.00 & 0.0 & 0.33 & - & [123] \\
\hline V & 1.82 & -0.49 & 0.77 & - & - & - & [124] \\
\hline $\mathrm{Cr}$ & 0.94 & 2.56 & -0.54 & 16.5 & 0.31 & - & [122] \\
\hline \multirow[t]{3}{*}{ Mn } & 0.66 & -2.93 & 1.18 & - & - & - & [125] \\
\hline & 1.30 & -2.38 & 1.23 & - & - & - & [126] \\
\hline & 9.83 & 3.27 & 2.18 & 23.9 & - & - & [128] \\
\hline \multirow[t]{2}{*}{$\mathrm{Fe}$} & 10.18 & 2.90 & 2.32 & 22.3 & - & 0.24 & [129] \\
\hline & 9.80 & 2.84 & 2.27 & - & - & - & [130] \\
\hline \multirow[t]{3}{*}{ Co } & 8.92 & 2.38 & 1.78 & - & - & - & [135] \\
\hline & 8.77 & 2.32 & 1.76 & - & - & - & [136] \\
\hline & 8.47 & 2.26 & 2.07 & 23.1 & 0.56 & - & [123] \\
\hline \multirow[t]{5}{*}{$\mathrm{Ni}$} & 7.22 & 2.20 & 0.62 & - & - & - & [108] \\
\hline & 7.37 & 2.16 & 0.86 & 17.4 & - & - & [139] \\
\hline & 7.45 & 2.17 & 0.94 & 27.9 & 0.33 & - & [120] \\
\hline & 7.90 & 2.44 & 0.58 & - & - & - & [139] \\
\hline & 7.27 & 2.14 & 0.85 & - & - & - & [141] \\
\hline $\mathrm{Cu}$ & 6.18 & 1.97 & 0.30 & 27.4 & 0.29 & - & [111] \\
\hline $\mathrm{Zn}$ & 3.60 & 1.21 & - & 13.2 & 0.34 & - & [113] \\
\hline \multirow[t]{3}{*}{$\mathrm{Ru}$} & 6.38 & 0.68 & 1.89 & 13.4 & - & - & [142] \\
\hline & 7.66 & 0.74 & 2.30 & - & - & - & [143] \\
\hline & 7.97 & 0.70 & 2.40 & 15.1 & - & - & [144] \\
\hline \multirow[t]{3}{*}{$\mathrm{Rh}$} & 9.20 & 0.96 & 2.76 & - & - & - & [137] \\
\hline & 5.52 & 0.94 & 2.70 & - & - & - & [135] \\
\hline & 9.20 & - & - & - & - & - & [116] \\
\hline \multirow[t]{3}{*}{$\mathrm{Pd}$} & 8.11 & 0.44 & 2.54 & 15.5 & - & - & [138] \\
\hline & 8.27 & 0.38 & 2.68 & - & - & - & [118] \\
\hline & 7.47 & 1.51 & 2.00 & - & - & - & [125] \\
\hline $\mathrm{Ag}$ & 6.14 & 0.48 & 1.90 & 23.4 & 0.29 & - & [145] \\
\hline
\end{tabular}


Adicionalmente, también se ha investigado la dependencia de las propiedades estructurales y magnéticas, respecto a la concentración $(x)$ del átomo $M$, en los compuestos ternarios tipo $M_{x} \mathrm{Fe}_{4-x} N(0 \leq x \leq 1)$, tanto de forma experimental, $M=$ Mn [12], Ni [57,103-107,109,119,121,122,146,147], Co [104,148], Cu [111,112], Zn [113], Ru [113], Ag [145], Ga [149-151], In [113, 152] y Sn [153, 154]; como también desde el punto de vista teórico, $M=\mathrm{Ni}[120,139,140]$, Ga [149,156], In [152]; existiendo aún muy poca bibliografía teórico de estos compuestos.

\subsection{Metodología}

Todos los cálculos han sido realizados dentro del contexto de la Teoría de la Funcional Densidad, usando el método FP-LAPW el cual ha sido implementado en el código Wien2k [157]. Para la parte de intercambio y correlación, se consideró la funcional propuesta por J.P. Perdew, S. Burke y M. Ernzerhof (PBE) [34], la cual ha sido desarrollada dentro del contexto de GGA. Además, se han hecho las siguientes consideraciones.

- Todos los cálculos han sido convergidos usando el criterio de que la variación de la densidad de carga sea de $10^{-5}$ u.a.

- Polarización de espín para los cálculos magnéticos.

- El parámetro $R_{M T} \times K_{\max }$ igual a 8 , mientras que el parámetro $G_{\max }=15$ u.a.

- Los radios de muffin-tin usados son:1.79 bohr para el Fe, 2.59 bohr para los átomos $X$ ( $X$ es un metal 3d, $4 d \mathrm{o} \mathrm{Al}, \mathrm{Ga}, \mathrm{In}) \mathrm{y} 1.59$ bohr para el $\mathrm{N}$.

- Efectos relativistas para los electrones del carozo (full relativístico) y de valencia (escalar relativístico)

- El espacio recíproco fue descrito por una grilla de $21 \times 21 \times 21$ puntos $k$ para el caso de los compuestos $X \mathrm{Fe}_{3} \mathrm{~N}$, mientras que para el caso de superceldas se usó una grilla de $9 \times 9 \times 9$ puntos $k$. 


\subsubsection{Ecuación de Estado de Birch-Murnaghan}

Para la obtención del volumen $\left(V_{0}\right)$ como el módulo de bulk $(B)$ de equilibrio a partir de los datos de la energía total $(E)$ calculada para diferentes volúmenes $(V)$, se usará en todos los casos la ecuación de estado propuesta por Birch-Murnaghan de tercer orden $[158,159]$. Donde a partir de la relación propuesta entre la presión $(P)$ y el volumen $(V)$, ecuación (2.1), se puede determinar la relación existente entre la energía y el volumen (2.2)

$$
\begin{gathered}
P(V)=\frac{3 B}{2}\left[\left(\frac{V_{0}}{V}\right)^{\frac{7}{3}}-\left(\frac{V_{0}}{V}\right)^{\frac{5}{3}}\right]\left\{1+\frac{3}{4}\left(B^{\prime}-4\right)\left[\left(\frac{V_{0}}{V}\right)^{\frac{2}{3}}-1\right]\right\} \\
E(V)=E_{0}+\frac{9 V_{0} B}{16}\left\{\left[\left(\frac{V_{0}}{V}\right)^{\frac{2}{3}}-1\right]^{3} B^{\prime}+\left[\left(\frac{V_{0}}{V}\right)^{\frac{2}{3}}-1\right]^{2}\left[6-4\left(\frac{V_{0}}{V}\right)^{\frac{2}{3}}\right]\right\}
\end{gathered}
$$

donde $E_{0}$ es la energía correspondiente al volumen de equilibrio, $B^{\prime}$ es la derivada respecto a la presión del módulo de bulk.

\subsubsection{Aproximación de la Supercelda}

Para el estudio de los compuestos ternarios tipo $M_{x} \mathrm{Fe}_{4-x} \mathrm{~N}(0 \leq x \leq 1)$ se usó una supercelda de $2 \times 2 \times 2$ de $\gamma^{\prime}-\mathrm{Fe}_{4} \mathrm{~N}$ (Figura 2.2a) en la cual existen 8 átomos FeI, 24 átomos FeII y 8 átomos de N. De esta supercelda, se puede apreciar que los átomos FeI forman una subred cúbica (Figura 2.2b), para lo cual sustituyendo distintas cantidades de átomos $\mathrm{Fe} I$ por átomos $M$ es posible obtener distintos valores de $(x)$, esto es, al sustituir $0,1,2,3,4$, 5, 6, 7 u 8 átomos de FeI por átomos $M$ se obtendrán los siguientes valores de $x=0.000,0.125,0.250,0.375,0.500,0.625$, $0.750,0.875,1.00$, respectivamente. Cabe mencionar que para $x=0.000$ se obtiene el compuesto $\gamma^{\prime}-\mathrm{Fe}_{4} \mathrm{~N}$, mientras que para $x=1.000$, es decir los 8 átomos FeI han sido sustituidos por átomos $M$, se obtiene el compuesto ternario $M \mathrm{Fe}_{3} \mathrm{~N}$. Para el caso de concentraciones intermedias existe más de una configuración no equivalente posible de obtener un mismo valor de $x$, por lo cual se usara la nomenclatura 
$C_{i \ldots j}^{k}$ para indicar las distintas configuraciones posibles para un determinado valor de $x$, donde el superíndice $k$ indica la cantidad de átomos FeI sustituidos por átomos $M$, mientras que el subíndice $i \ldots j$ representa que átomos FeI, que conforman la subred cúbica (Figura 2.2b), han sido sustituidos. En La Tabla 2.7 se muestran las distintas configuraciones no equivalentes posibles que han sido consideradas para cada valor de $x$. Mientras que en la Figura 2.3 se muestran los esquemas de las diferentes configuraciones consideradas en la Tabla 2.7

Tabla 2.7: Razón entre la cantidad de átomos $M$ y FeI $(M$ :FeI) en la supercelda $2 \times 2 \times 2$ de $\gamma^{\prime}-\mathrm{Fe}_{4} \mathrm{~N}$ para obtener diferentes valores de concentración $x$ en los compuestos $M_{x} \mathrm{Fe}_{4-x} \mathrm{~N}$, con sus respectivas configuraciones no equivalente.

\begin{tabular}{ccll}
\hline$M: F e I$ & $x$ & Fórmula & Configuraciones \\
\hline $0: 8$ & 0.000 & $\gamma^{\prime}-\mathrm{Fe}_{4} \mathrm{~N}$ & - \\
$1: 8$ & 0.125 & $M_{0.125} \mathrm{Fe}_{3.875} \mathrm{~N}$ & $\mathrm{C}_{1}^{1}$ \\
$2: 8$ & 0.250 & $M_{0.250} \mathrm{Fe}_{3.750} \mathrm{~N}$ & $\mathrm{C}_{12}^{2}, \mathrm{C}_{23}^{2}, \mathrm{C}_{18}^{2}$ \\
$3: 8$ & 0.375 & $M_{0.375} \mathrm{Fe}_{3.625} \mathrm{~N}$ & $\mathrm{C}_{123}^{3}, \mathrm{C}_{267}^{3}, \mathrm{C}_{258}^{3}$ \\
$4: 8$ & 0.500 & $M_{0.500} \mathrm{Fe}_{3.500} \mathrm{~N}$ & $\mathrm{C}_{1234}^{4}, \mathrm{C}_{1347}^{4}, \mathrm{C}_{1345}^{4}, \mathrm{C}_{1346}^{4}, \mathrm{C}_{1368}^{4}, \mathrm{C}_{1467}^{4}$ \\
$5: 8$ & 0.625 & $M_{0.625} \mathrm{Fe}_{3.375} \mathrm{~N}$ & $\mathrm{C}_{12345}^{5}, \mathrm{C}_{13458}^{5}, \mathrm{C}_{13467}^{5}$ \\
$6: 8$ & 0.750 & $M_{0.750} \mathrm{Fe}_{3.250} \mathrm{~N}$ & $\mathrm{C}_{123456}^{6}, \mathrm{C}_{123458}^{6}, \mathrm{C}_{123678}^{6}$ \\
$7: 8$ & 0.875 & $M_{0.875} \mathrm{Fe}_{3.125} \mathrm{~N}$ & $\mathrm{C}_{234567}^{7}$ \\
$8: 8$ & 1.000 & $M_{1.000} \mathrm{Fe}_{3.000} \mathrm{~N}$ & $\mathrm{C}_{12345678}^{8}$ \\
\hline
\end{tabular}




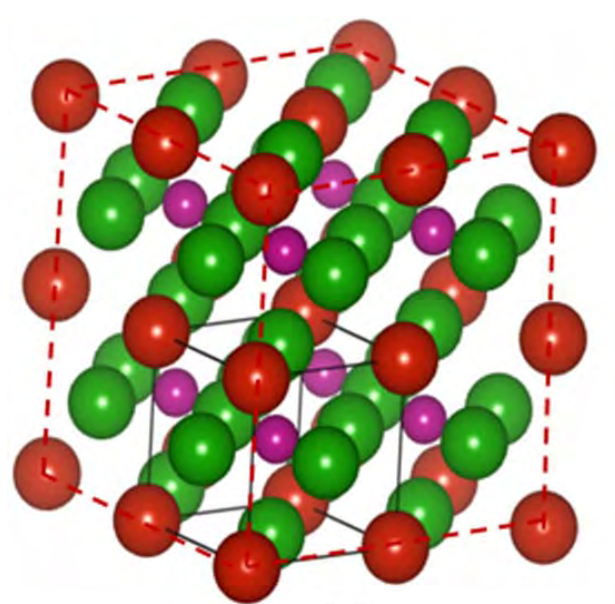

(a)

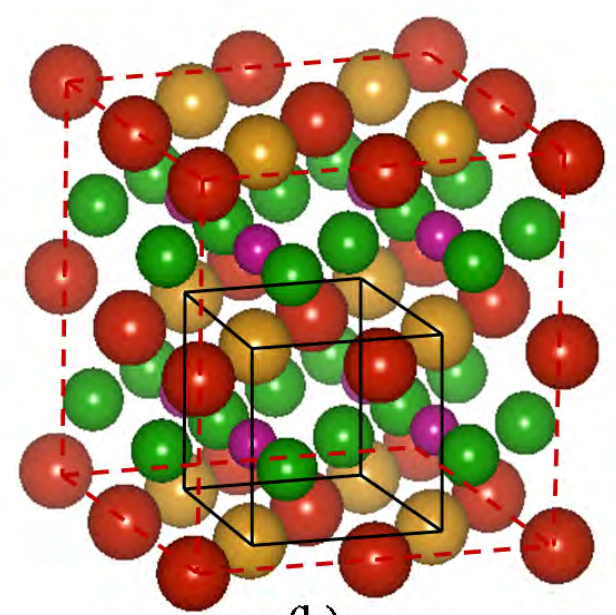

(b)

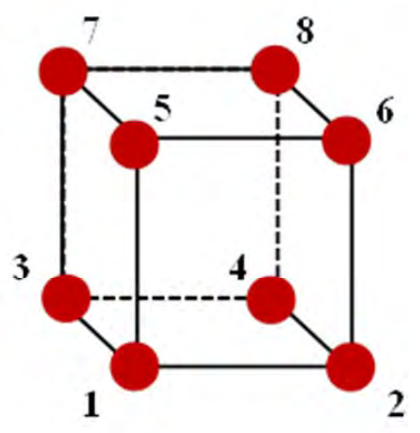

(c)

Figura 2.2: Supercelda $2 \times 2 \times 2$ de $\gamma^{\prime}-\mathrm{Fe}_{4} \mathrm{~N}$ utilizada para obtener diferentes valores de $x$ en los compuestos ternarios $M_{x} \mathrm{Fe}_{4-x} \mathrm{~N}$ para el caso que a) el átomo $M$ sustituye a un $\mathrm{FeI}, \mathrm{b}$ ) el átomos $M$ sustituye a un FeII y c) subred cúbica formada por los 8 átomos de $\mathrm{Fe} I \mathrm{o} F e I I$ que podrán ser reemplazados por un átomo $M$.

Para el caso de compuestos ternarios $M_{x} \mathrm{Fe}_{4-x} \mathrm{~N}$, donde $M$ sustituye a uno de los átomos FeII del $\gamma^{\prime}-\mathrm{Fe}_{4} \mathrm{~N}$, por ejemplo el FeII que se encuentra en la base de la celda cúbica del $\gamma^{\prime}-\mathrm{Fe}_{4} \mathrm{~N}$, en la supercelda $2 \times 2 \times 2$ de $\gamma^{\prime}-\mathrm{Fe}_{4} \mathrm{~N}$ dichos átomos FeII también formaran una subred cúbica (Figura 2.2b) semejante al caso descrito anteriormente, el átomos $M$ sustituye a un FeI (Figura 2.2a). Además, no se han considerado las posibilidades de que dos o más átomos de $M$ puedan sustituir a 
los FeII de una misma celda unitaria de $\gamma^{\prime}-\mathrm{Fe}_{4} \mathrm{~N}$, ni tampoco el caso de sustituciones aleatorias de átomos FeI y FeII simultáneamente. Por lo tanto, el estudio de estas superceldas será semejante a las planteadas anteriormente, siguiendo así las mismas configuraciones mostradas en la Tabla 2.7 y en la Figura 2.3.

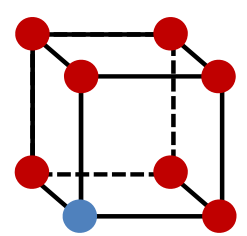

$\mathrm{C}_{1}^{1}$

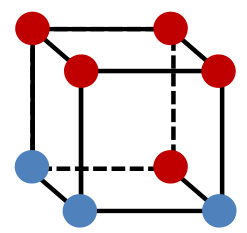

$\mathrm{C}^{3}{ }_{123}$

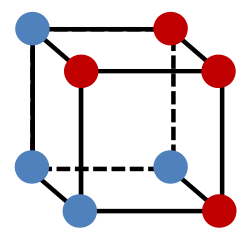

$\mathrm{C}^{4}{ }_{1347}$

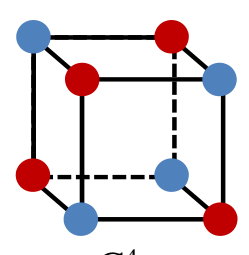

$\mathrm{C}^{4}{ }_{1467}$

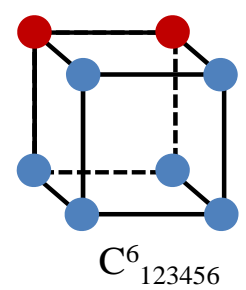

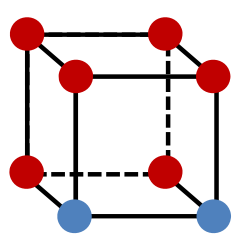

$\mathrm{C}^{2} 12$

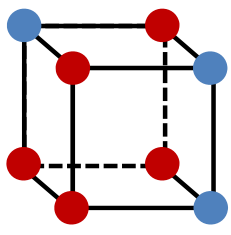

$\mathrm{C}^{3}{ }_{267}$

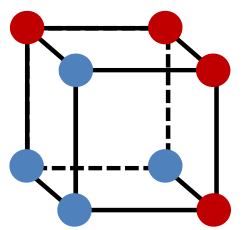

$\mathrm{C}^{4}{ }_{1345}$
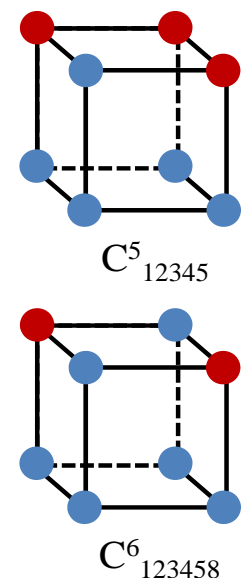

$\mathrm{C}^{6}{ }_{123458}$

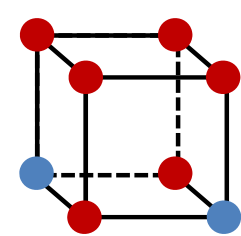

$\mathrm{C}^{2}{ }_{23}$

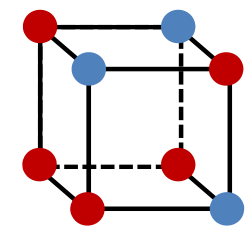

$\mathrm{C}^{3}{ }_{258}$
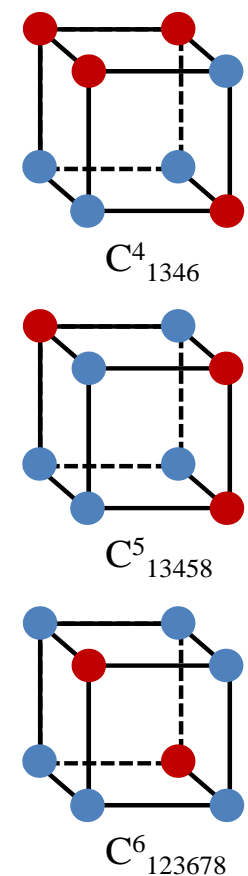

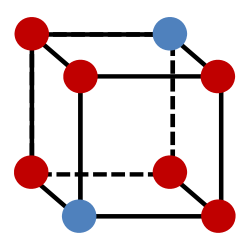

$\mathrm{C}^{2}{ }_{18}$

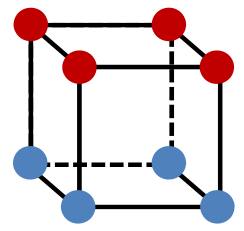

$\mathrm{C}^{4}{ }_{1234}$

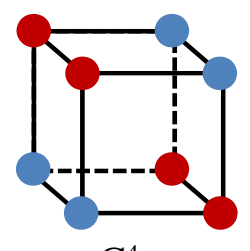

$\mathrm{C}^{4}{ }_{1368}$
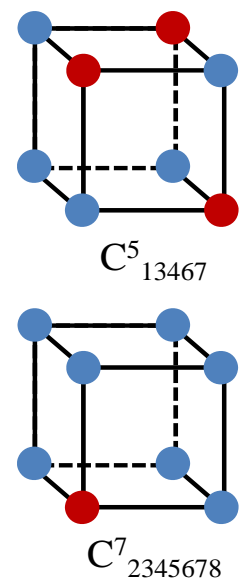

Figura 2.3: Distintas configuraciones $C_{i \ldots j}^{k}$ correspondientes a las diferentes concentraciones $x$. 


\subsection{Cálculo de las Propiedades Hiperfinas con el Método FP-LAPW}

- Campo Hiperfino: $\left(B_{h f}\right)$

El campo hiperfino $\left(B_{h f}\right)$ puede ser expresado como la suma de tres componentes $[160,161]$ :

$$
\mathbf{B}_{h f}=\mathbf{B}_{d}+\mathbf{B}_{o}+\mathbf{B}_{c}
$$

donde $\mathbf{B}_{d}$ es el término dipolar, $\mathbf{B}_{o}$ es el término orbital y $\mathbf{B}_{c}$ es el término de contacto de Fermi. Debido a la poca contribución del $\mathbf{B}_{d} \mathbf{y} \mathbf{B}_{o}(\sim 2 T)$ al $\mathbf{B}_{h f}$ frente al $\mathbf{B}_{c}(\sim 33 T)$, en el desarrollo del presente trabajo se considerará tan sólo la contribución del $\mathbf{B}_{c}$ [162,163]:

$$
\mathbf{B}_{h f}=\frac{8 \pi}{3} \mu_{B}\left[\rho_{\uparrow}(0)-\rho_{\downarrow}(0)\right]
$$

donde $\mu_{B}$ es el magnetón de Bohr y la expresión entre corchetes representa la diferencia de las densidades de estados electrónicos up y down cercanos al núcleo.

- Corrimiento Isomérico: $(\delta)$

Los valores del corrimiento isomérico $(\delta)$ serán obtenidos teniendo en cuenta la expresión [160,161]:

$$
\delta=\alpha\left[\rho_{a}(0)-\rho_{s}(0)\right]
$$

donde $\rho_{a}(0)$ y $\rho_{s}(0)$ representan la densidad de carga electrónica cercana al núcleo del adsorbente $(a)$ y la fuente $(s), \alpha-\mathrm{Fe}(\mathrm{BCC})$, mientras que $\alpha$ es una constante de calibración cuyo valor considerado en el presenta trabajo será $\alpha=-0.22 a_{0}^{-3} \mathrm{~mm} / \mathrm{s}$ [164].

\section{- Desdoblamiento Cuadrupolar: $(\varepsilon)$}

Será determinado usando la relación [160]:

$$
\varepsilon=\frac{e Q_{N}}{2} V_{z z}\left(1+\frac{\eta^{2}}{3}\right)^{1 / 2}
$$


donde $e$ es la carga del electron, $Q_{N}$ es el momento cuadrupolar nuclear del estado excitado del ${ }^{57} \mathrm{Fe}$ de $14.4 \mathrm{keV}$ cuyo valor es $Q_{N}=-0.16 \times 10^{-28} \mathrm{~m}^{2}$ [165], $V_{z z}$ es el componente principal del tensor gradiente de campo eléctrico (EFG) y $\eta$ es el parámetro de asimetría [160], estos dos últimos parámetros se obtienen de los cálculos ab-initio.

\section{- Constante de Acoplamiento Hiperfino: $(A)$}

El término de contacto de Fermi $\mathbf{B}_{c}$ de (2.3) consiste principalmente en la contribución de dos componentes [166]:

$$
\mathbf{B}_{c}=\mathbf{B}_{\text {core }}+\mathbf{B}_{v a l}
$$

donde $\mathbf{B}_{\text {core }}$ representa el campo hiperfino debido a la polarización de los electrones del core $(1 s, 2 s, 3 s)$. Mientras que $\mathbf{B}_{v a l}$ representa el campo hiperfino debido a la polarización de los electrones de la banda de valencia (4s, 3d, $4 p$ ). El primer término es proporcional al momento magnético de los orbitales $3 d\left(\mu_{d}\right)[166]$, obteniéndose así:

$$
\mathbf{B}_{h f}=A \mu_{d}+b\left[\rho_{\uparrow}^{v a l}(0)-\rho_{\downarrow}^{v a l}(0)\right]
$$

donde $\rho_{\uparrow}^{\text {val }}(0)$ y $\rho_{\downarrow}^{v a l}(0)$ son las densidades electrónicas de contacto procedentes de las bandas de valencia, mientras que $A$ y $b$ son constantes de proporcionalidad. De (2.8) se observa una relación lineal $[167,168]$ entre el $\mathbf{B}_{h f}$ y el $\mu_{d}$ a través de la denominada constante de acoplamiento hiperfino $(A)$, la cual ha sido determinada de forma teórica [169-171] como experimental [172-174] en diferentes compuestos ferrosos. 


\section{Capítulo 3}

\section{Sistemas Ternarios $X \mathrm{Fe}_{3} \mathrm{~N}(X$ un}

\section{metal $3 d$ o $4 d$ )}

En el presente capítulo se realizará un estudio sistemático de la variación de las propiedades estructurales, magnéticas e hiperfinas del $\gamma^{\prime}-\mathrm{Fe}_{4} \mathrm{~N}$ al sustituir un átomo de Fe por un átomo $X$ para formar así los compuestos ternarios $X \mathrm{Fe}_{3} \mathrm{~N}$, este estudio se realizará para el caso de que el átomo $X$ sustituya al átomo $\mathrm{FeI} o$ a un FeII , el cual nos ayudara a analizar la existencia de algún sitio preferencial de sustitución del átomo $X$ en el compuesto $\gamma^{\prime}-\mathrm{Fe}_{4} \mathrm{~N}$. Dichos resultados serán comparados con los valores experimentales y teóricos recopilados de la bibliografía (Tablas del Capítulo 2), complementándose así con el análisis e interpretación de los resultados obtenidos en el presente trabajo.

Considerando que el $\gamma^{\prime}-\mathrm{Fe}_{4} \mathrm{~N}$ posee una estructura cristalina cúbica tipo antiperovskita, en la cual es posible distinguir dos átomos de Fe, FeI y FeII ubicados respectivamente en la esquina y en las caras de la celda cúbica [47-50], existen dos alternativas para obtener los compuestos ternarios $X \mathrm{Fe}_{3} \mathrm{~N}$. Por un lado considerando que el átomo $X$ sustituye al FeI (sitio 1a de Wyckoff) el cual es isoestructural al $\gamma^{\prime}-\mathrm{Fe}_{4} \mathrm{~N}$ (Figura 3.1a) y por otro lado, que el átomo de $X$ sustituye un átomo FeII (sitio $3 c$ de Wyckoff) formando así otra estructura cristalina con grupo espacial P4/mmm (Figura 3.1b) el cual se caracteriza por ser una estructura cristalina tetragonal donde dos parámetros de red son iguales $(a$ y $b)$ y distinto al tercero (c), $a=b \neq c$. Para el compuesto $\gamma^{\prime}-\mathrm{Fe}_{4} \mathrm{~N}$, tanto la estructura cristalina $P m \overline{3} m$ y 
$P 4 / m m m(\operatorname{con} c / a=1)$ son semejantes.

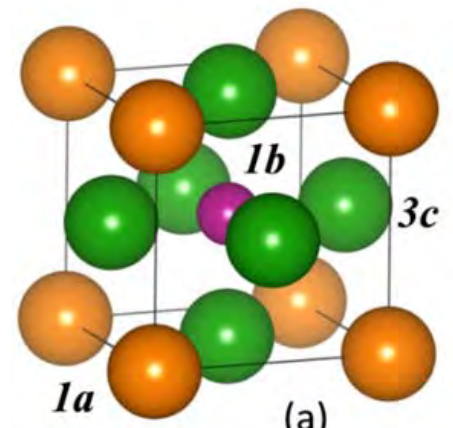

(a)

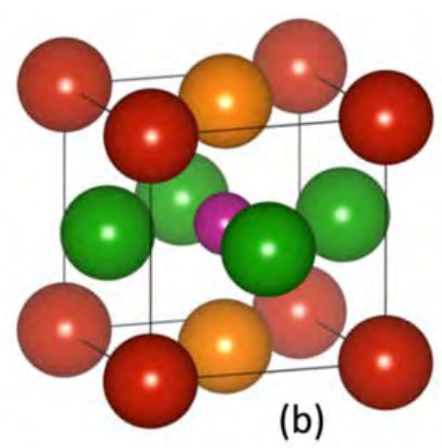

(b)

Figura 3.1: Estructuras cristalinas a) $P m \overline{3} m$ y b) $P 4 / m m m$ de los compuestos ternarios $\mathrm{XFe}_{3} \mathrm{~N}$.

El desarrollo del presente capítulo se dividirá en dos partes principales, la primera estará dedicada al estudio de los compuestos ternarios $X \mathrm{Fe}_{3} \mathrm{~N}$ cuando $X$ es un metal de transición de la serie $3 d$, mientras que la segunda parte será dedicada para el caso que $X$ es un metal de transición de la serie $4 d$.

\subsection{Compuestos Ternarios $X \mathrm{Fe}_{3} \mathrm{~N}$ ( $X$ metal $\left.3 d\right)$}

\subsubsection{Sitio de sustitución preferencial del átomo $X$}

En esta primera parte se estudiará la variación de las propiedades estructurales, magnéticas e hiperfinas del $\gamma^{\prime}-\mathrm{Fe}_{4} \mathrm{~N}$ al sustituir un átomo de Fe por un átomo $X$, donde $X$ puede ser Sc, V, Ti, Cr, Mn, Co, Ni, Cu o Zn. Para lo cual se han considerado los dos casos posibles de sustitución, las estructuras $P m \overline{3} m$ y $P 4 / m m m$. En cada tipo de estructura se han realizado cálculos autoconsistentes para cada uno de los diferentes valores de volumen $(V)$ del compuesto, obteniéndose la energía total $(E)$ para cada uno de ellos. Dichos datos se ajustaron con la ecuación de estado de Birch-Murnaghan (2.2) de tercer orden, permitiendo determinar el volumen de equilibrio $\left(V_{0}\right)$, el módulo de bulk del compuesto $(B)$ y la derivada del módulo de bulk respecto de la presión $\left(B^{\prime}\right)$. En la Figura 3.2 se muestran las curvas de 
energía total en función del volumen de la celda cristalina para ambas estructuras cristalinas del compuesto ternario $\mathrm{XFe}_{3} \mathrm{~N}$.

A partir de los valores mínimos de las energías totales obtenidas del ajuste $E-V$, se obtuvo la diferencia $\Delta E=E_{0}(P m \overline{3} m)-E_{0}(P 4 / m m m)$ con el fin de determinar cuál de las dos estructuras es la más estable. En la Tabla 3.1 se muestran los valores de $\left(V_{0}\right)$ y $(B)$ obtenidos de dichos ajustes para ambos tipos de estructuras cristalinas, junto con la $\Delta E$ entre dichas estructuras.

Tabla 3.1: Volumen de equilibrio $\left(V_{0}\right)$ y el módulo de bulk $(B)$ obtenidos del ajuste con la ecuación de estado de Birch-Murnaghan, para ambas estructuras cristalinas del compuesto ternario $\mathrm{XFe}_{3} \mathrm{~N}$. Además, la diferencia de la energía total $(\Delta E)$ entre ambas estructuras en su respectivo $V_{0}$.

\begin{tabular}{cccccc}
\hline \multicolumn{4}{c}{$V_{0}\left(\AA^{3}\right)$} & \multicolumn{2}{c}{$B(\mathrm{GPa})$} \\
$\mathrm{X}$ & $P m \overline{3} m$ & $P 4 / m m m$ & $P m \overline{3} m$ & $P 4 / m m m$ & $\Delta E$ \\
\hline $\mathrm{Sc}$ & 59.133 & 63.868 & 121.0 & 141.4 & -23.60 \\
$\mathrm{Ti}$ & 53.186 & 58.585 & 230.1 & 168.6 & 0.58 \\
$\mathrm{~V}$ & 53.794 & 55.027 & 137.0 & 162.2 & 37.70 \\
$\mathrm{Cr}$ & 55.429 & 54.289 & 135.1 & 174.5 & 34.67 \\
$\mathrm{Mn}$ & 56.185 & 54.968 & 176.4 & 170.2 & 11.00 \\
$\mathrm{Fe}$ & 54.440 & 54.440 & 175.1 & 175.1 & 0.00 \\
$\mathrm{Co}$ & 53.943 & 53.630 & 187.0 & 175.9 & -1.65 \\
$\mathrm{Ni}$ & 54.060 & 54.076 & 192.1 & 207.0 & -13.22 \\
$\mathrm{Cu}$ & 54.546 & 55.590 & 159.6 & 192.2 & -58.24 \\
$\mathrm{Zn}$ & 54.271 & 57.182 & 163.0 & 169.6 & -126.45 \\
\hline
\end{tabular}




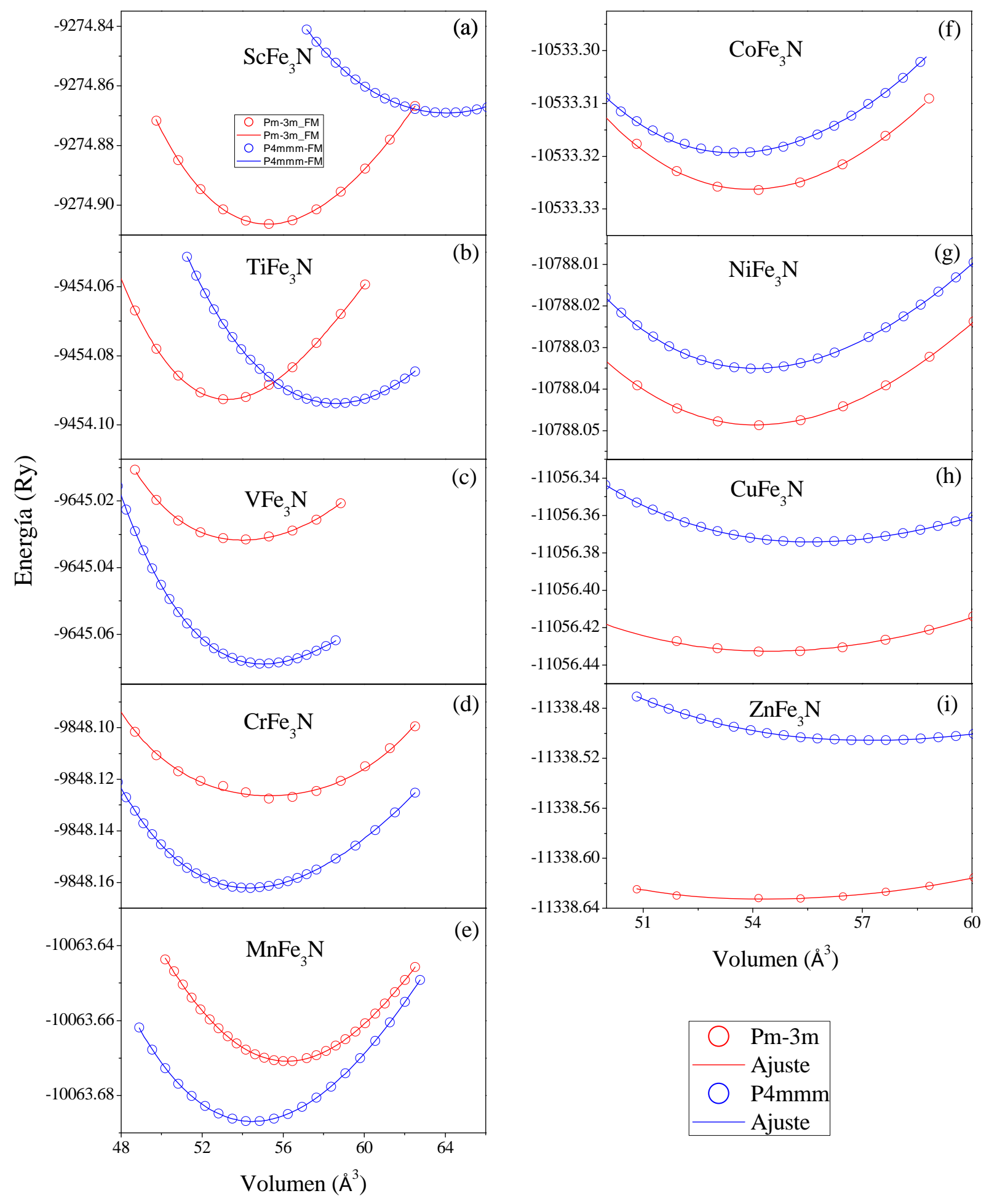

Figura 3.2: Curvas volumen - energía para los compuestos ternarios: a) $\left.\mathrm{ScFe}_{3} \mathrm{~N}, \mathrm{~b}\right) \mathrm{TiFe}_{3} \mathrm{~N}$, c) $\mathrm{VFe}_{3} \mathrm{~N}$, d) $\left.\left.\left.\left.\left.\mathrm{CrFe}_{3} \mathrm{~N}, \mathrm{e}\right) \mathrm{MnFe}_{3} \mathrm{~N}, \mathrm{f}\right) \mathrm{CoFe}_{3} \mathrm{~N}, \mathrm{~g}\right) \mathrm{NiFe}_{3} \mathrm{~N}, \mathrm{~h}\right) \mathrm{CuFe}_{3} \mathrm{~N}, \mathrm{i}\right) \mathrm{ZnFe}_{3} \mathrm{~N}$ para las estructuras cristalinas $P m \overline{3} m$ y $P 4 / m m m$. 
En la Figura 3.3 se muestra el valor $\Delta E$ para los diferentes átomos $X$, por un lado tenemos que para $X=\mathrm{Sc}, \mathrm{Co}, \mathrm{Ni}, \mathrm{Cu}$ y $\mathrm{Zn}$ la estructura cristalina de mínima energía es la $\operatorname{P} m \overline{3} m$ lo cual indica que estos átomos prefieren sustituir a un átomo $\mathrm{Fe} I$ concordando así con medidas experimentales realizadas para $X=\mathrm{Ni}$ [49,53,103-110], $\mathrm{Cu}[111,112]$ y $\mathrm{Zn}[113,114]$. Para el caso de $X=\mathrm{Co}\left(\mathrm{CoFe}_{3} \mathrm{~N}\right)$ es posible distinguir una pequeña diferencia entre las dos estructuras cristalinas, lo cual no fue posible en [137]. Debido a la ausencia de datos experimentales, esta diferencia permite predecir el sitio preferencial de sustitución del Co en el compuesto $\gamma^{\prime}-\mathrm{Fe}_{4} \mathrm{~N}$, como también la estructura cristalina del compuesto ternario $\mathrm{CoFe}_{3} \mathrm{~N}$ $(P m \overline{3} m)$. Por otro lado, para $X=\mathrm{V}, \mathrm{Ti}, \mathrm{Cr}$ y $\mathrm{Mn}$, la estructura de mínima energía es la $P 4 / \mathrm{mmm}$. Indicando que estos átomos $X$ prefieren sustituir a un átomo FeII, esto difiere totalmente de los cálculos teóricos publicados donde siempre consideraron que los átomos de $\mathrm{V}, \mathrm{Ti}, \mathrm{Cr}$ y Mn sustituye a un átomo de FeI, formando así la estructura $\operatorname{Pm} \overline{3} m$ (Tabla 2.5). Nunca consideraron la posibilidad de la sustitución de un FeII por un átomo $X$. Tan sólo existen datos experimentales para el caso de $X=\mathrm{Mn}[12]$ en bajas concentraciones en donde no pudieron determinar la existencia de un sitio preferencial de sustitución del Mn, llegando así a la conclusión de que el átomo Mn puede sustituir tanto a un átomo $\mathrm{FeI}$ o FeII en el $\gamma^{\prime}-\mathrm{Fe}_{4} \mathrm{~N}$ de forma no preferencial. La ausencia de datos experimentales para los compuestos ternarios $\mathrm{VFe}_{3} \mathrm{~N}, \mathrm{TiFe}_{3} \mathrm{~N}$ y $\mathrm{CrFe}_{3} \mathrm{~N}$, nos permite caracterizarlos y/o predecir las propiedades físicas de dichos compuestos. 


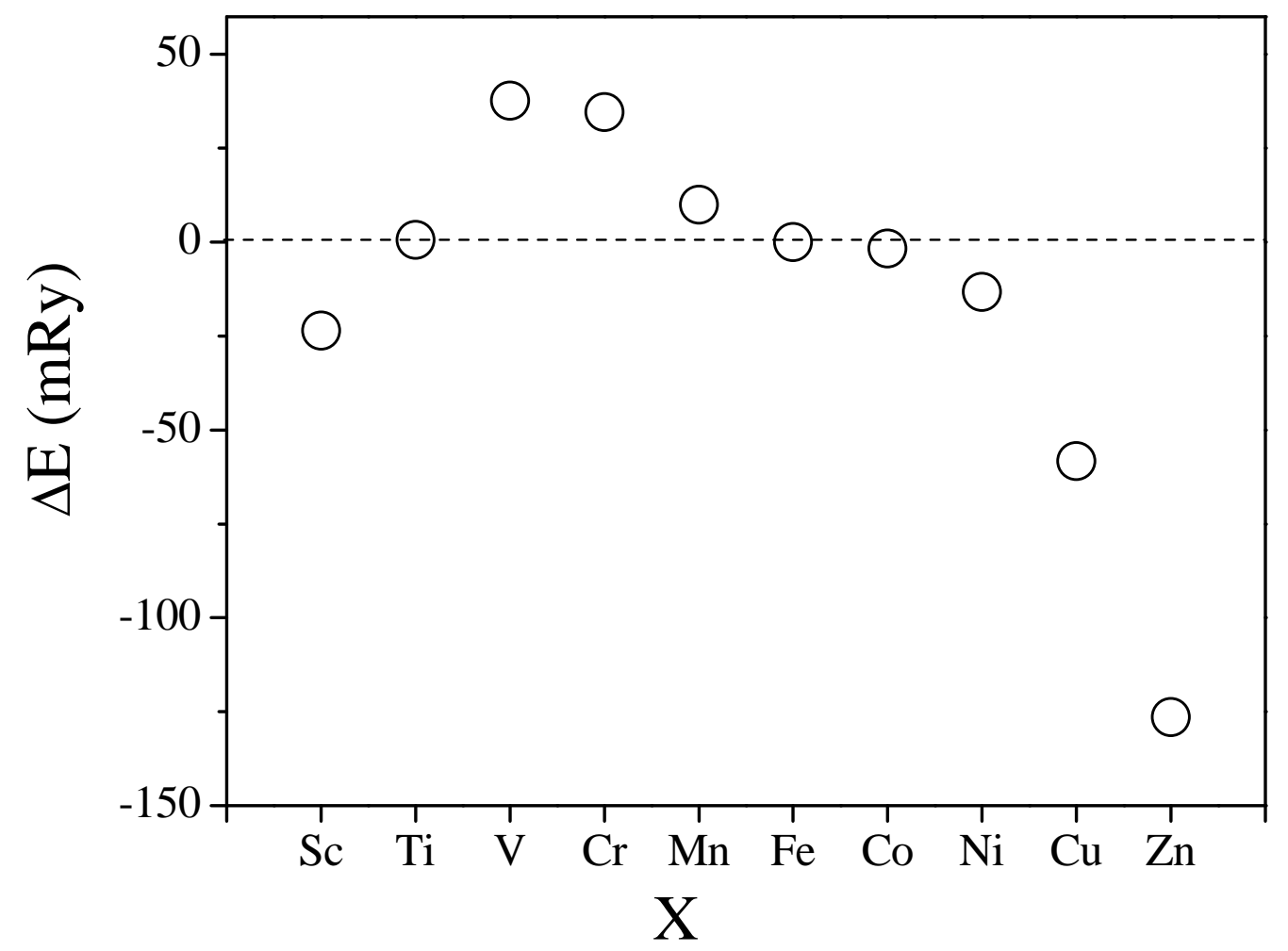

Figura 3.3: Valor de $\Delta E$ para cada compuesto ternario $X \mathrm{Fe}_{3} \mathrm{~N}$.

El análisis de las propiedades estructurales, magnéticas e hiperfinas de los compuestos ternarios $\mathrm{XFe}_{3} \mathrm{~N}$ se dividirá en dos partes. La primera parte será dedicada para el estudio de los compuestos ternarios con estructura cristalina $\operatorname{Pm} \overline{3} m$, mientras que la segunda parte será dedicada a los que tienen estructura cristalina $P 4 / \mathrm{mmm}$.

\subsubsection{Compuestos Ternarios $X \mathrm{Fe}_{3} \mathbf{N}$ con Estructura $P m \overline{3} m$}

\section{Propiedades Estructurales}

La sustitución preferencial de los átomos $\mathrm{Co}, \mathrm{Ni}, \mathrm{Cu}$ y $\mathrm{Zn}$ en el sitio del FeI está relacionada con la afinidad química entre dichos átomos y el átomo de $\mathrm{N}$, esto es, en compuestos de hierro-nitrógeno ordenados, los metales $3 d$ ubicados a la derecha del Fe en la Tabla Periódica pueden sustituir a átomos de Fe que no tengan 
átomos de $\mathrm{N}$ como primeros vecinos $[113,151]$. En la estructura cúbica $P m \overline{3} m$ del $\gamma^{\prime}-\mathrm{Fe}_{4} \mathrm{~N}$ con parámetro de red $a$, los sitios cristalográficos ocupados por los átomos FeII tienen a 2 átomos de $\mathrm{N}$ como primeros vecinos $(n n)$ a $a / 2$ y 4 átomos FeI como segundos vecinos (nnn) a $\sqrt{2} a / 2$, mientras que el sitio cristalográfico ocupado por el átomo FeI posee 12 átomos FelI como primeros vecinos a $\sqrt{2} a / 2$ y 8 átomos de $\mathrm{N}$ como segundos vecinos a $\sqrt{3} a / 2$. Esto indica que el sitio ocupado por el átomo FeI tiene a los átomos de $\mathrm{N}$ más lejanos que el sitio ocupado por los átomos FeII, justificando así la sustitución preferencial de los átomos $\mathrm{Co}, \mathrm{Ni}, \mathrm{Cu}$ y Zn en el sitio cristalográfico del FeI. Además, cuando $X=\mathrm{Sc}\left(\mathrm{ScFe}_{3} \mathrm{~N}\right)$ se obtiene un compuesto con estructura $\operatorname{Pm} \overline{3} m$, motivo por el cual se analizará de forma independiente. Primero nos enfocaremos en el estudio cuando $X$ es un metal $3 \mathrm{~d}$, ubicados a la derecha del átomo de Fe en la Tabla Periódica y después para el caso del $\mathrm{ScFe}_{3} \mathrm{~N}$.

Teniendo en cuenta que estos compuestos poseen una estructura cúbica, es posible entonces obtener los valores del parámetro de red correspondiente a partir del volumen de equilibrio obtenido del ajuste de la curva energía total - volumen. En la Figura 3.4 se muestra el valor del parámetro de red, $a$, calculado en función del número atómico $(Z)$ del átomo $X$, observándose un comportamiento oscilatorio de $a$, variando levemente respecto del parámetro de red del $\gamma^{\prime}-\mathrm{Fe}_{4} \mathrm{~N}$, además se puede apreciar una buena concordancia con los valores experimentales obtenidos de la bibliografía (Tabla 2.3). Diferentes trabajos experimentales y/o teóricos proponen una dependencia directa entre la variación del $a$ de los compuestos $X F e_{3} \mathrm{~N}$, respecto al $\gamma^{\prime}-\mathrm{Fe}_{4} \mathrm{~N}$, con la diferencia de los radios metálicos $\left(r_{m e t}\right)[152,155,174]$ o atómico $\left(r_{a t o}\right)[12,113,154,174]$ entre el átomo de Fe y el átomo $X$. Pero nunca se realizó un estudio experimental y/o teórico sistemático de estos materiales que compruebe dicha relación. En la Figura 3.5, se muestran los valores de $a$ calculados junto con los valores experimentales obtenidos de la bibliografía (Tabla 2.3) respecto al $r_{m e t}$, observándose la ausencia de una relación directa entre $a$ y el $r_{m e t}$, la cual podría estar vinculada con otra contribución a la variación de $a$, posiblemente proveniente de la interacción magnética entre el átomo X y los FeII [130]. 


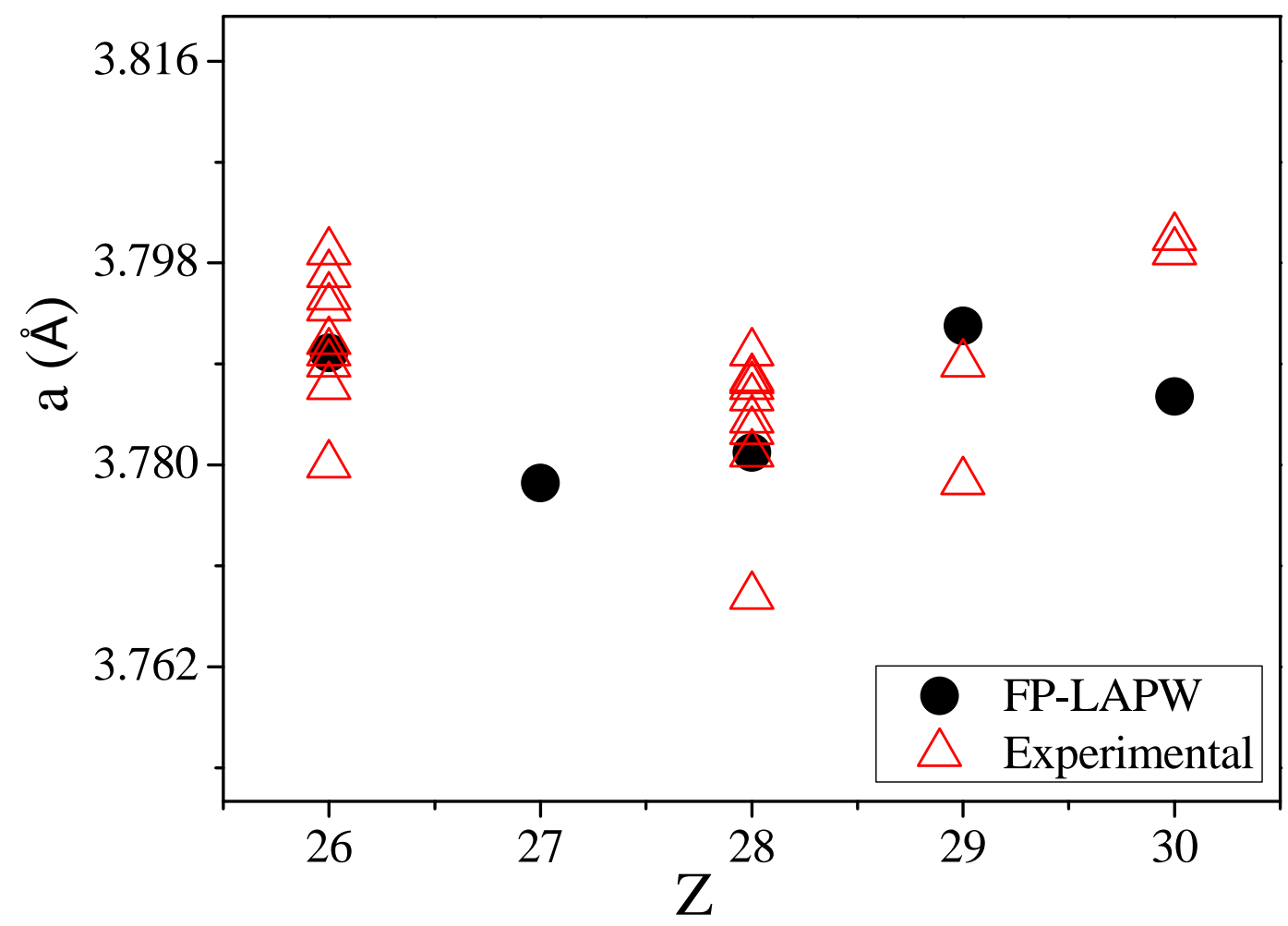

Figura 3.4: Variación del parámetro de red de los compuestos $X \mathrm{Fe}_{3} \mathrm{~N}$ en función del número atómico $(Z)$ del átomo $X$. 


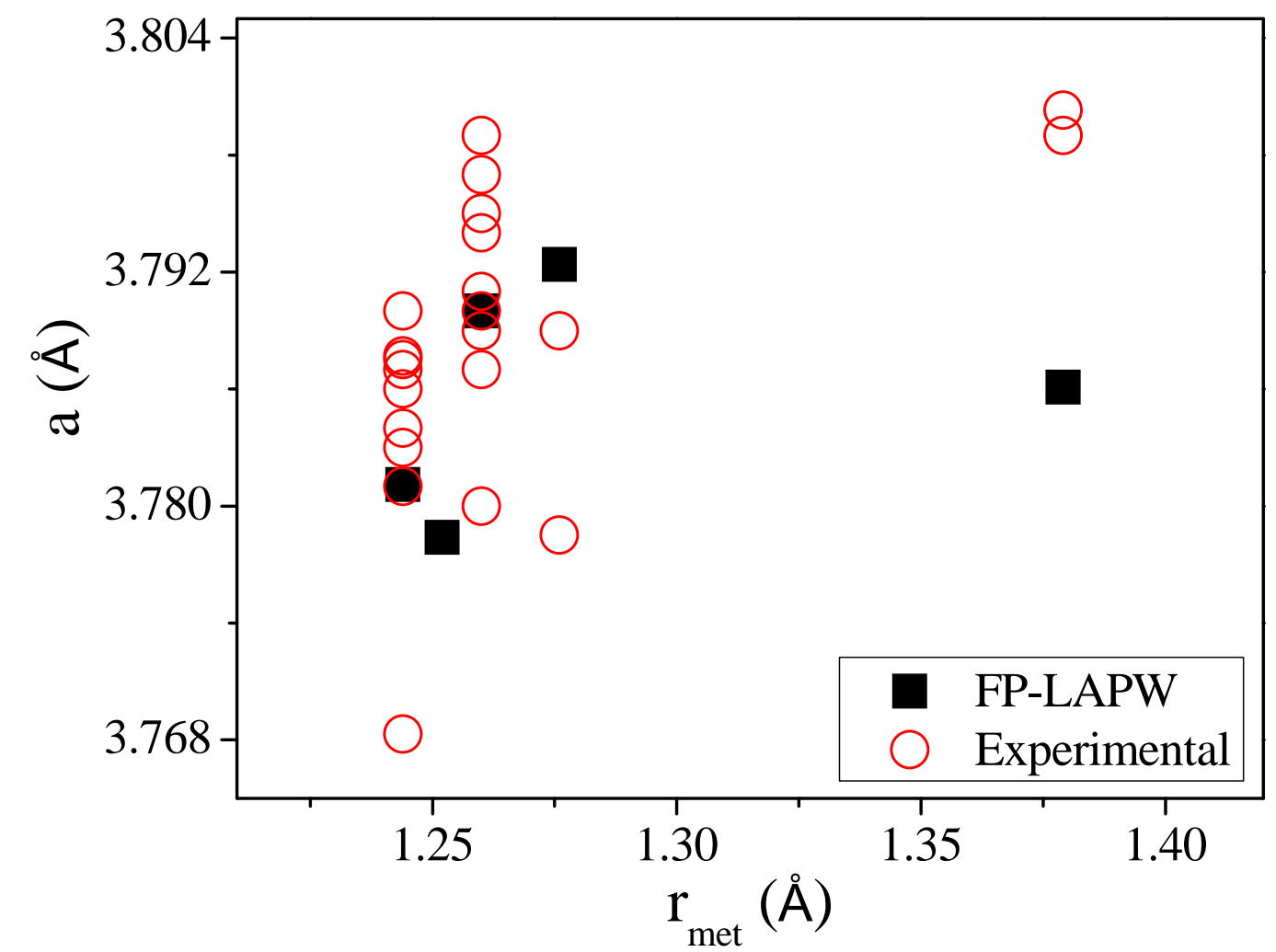

Figura 3.5: Variación del parámetro de red (a) de los compuestos ternarios $X \mathrm{Fe}_{3} \mathrm{~N}$ respecto al $r_{m e t}$ del átomo X.

Otro parámetro estructural que se puede obtener de la ecuación de estado de Birch-Murnaghan (2.2), es el módulo de bulk $(B)$, el cual está relacionado con la compresibilidad de los materiales. $\mathrm{El} \gamma^{\prime}-\mathrm{Fe}_{4} \mathrm{~N}$ es un material considerado dúctil y tolerante a daños debido a su alto valor de $B, 155$ GPa [63], 196 GPa [90]. Esta propiedad es mejorada al sustituir un átomo de $\mathrm{Fe}(\mathrm{Fe} I)$ por un átomo $X(X=\mathrm{Co}$, $\mathrm{Ni}, \mathrm{Cu}, \mathrm{Zn}$ ), incrementándose linealmente el valor de B (Figura 3.6), hasta un valor máximo para caso del $\mathrm{ZnFe}_{3} \mathrm{~N}$ donde se observa, además, un buen acuerdo con el valor experimental de $211.8 \mathrm{GPa}$ [114]. 


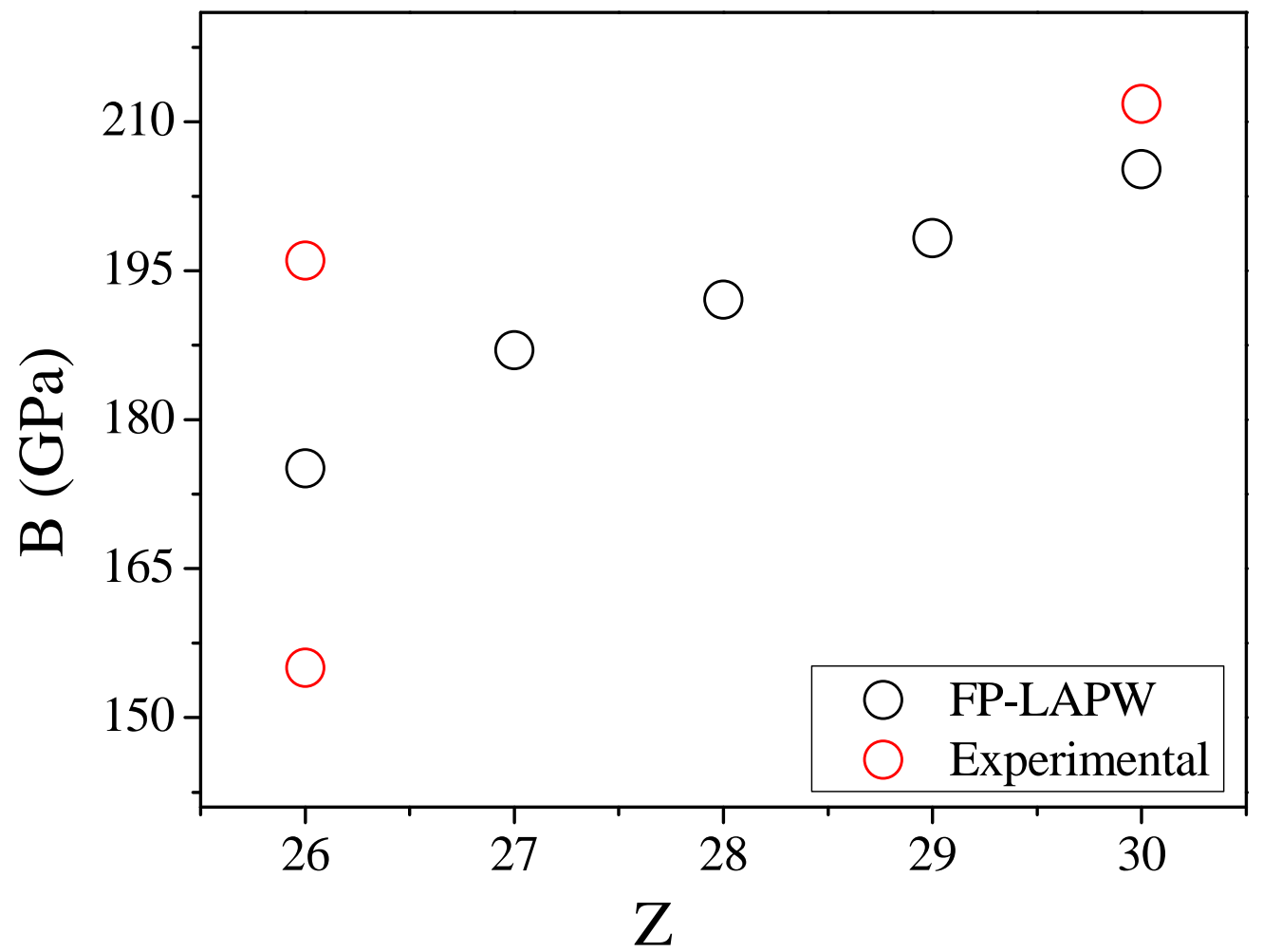

Figura 3.6: Dependencia del módulo de bulk $(B)$ con el número atómico $(Z)$ del átomo $X$, para los compuestos ternarios $\mathrm{XFe}_{3} \mathrm{~N}$. 


\section{Propiedades Magnéticas e Hiperfinas}

La sustitución del átomo FeI por un átomo $X$ en el $\gamma^{\prime}-\mathrm{Fe}_{4} \mathrm{~N}$ aparte de modificar la estructura cristalina también repercute en las propiedades magnéticas e hiperfinas. En la Tabla 3.2 se reportan los valores del momento magnético por fórmula unidad $\left(M_{f u}\right)$ y los momentos magnéticos de los átomos $X$ y FeII de los compuesto ternarios $X \mathrm{Fe}_{3} \mathrm{~N}$, mientras que en la Figura 3.7a se muestra el valor de $M_{f u}$ respecto del $Z$ del átomo $X$, observándose una dependencia lineal decreciente con una pendiente de $-1.14 \pm 0.06$, esta dependencia también se observa con los datos experimentales recopilados en la Tabla 2.4 cuya pendiente es de $-1.07 \pm 0.05$. La disminución de $M_{f u}$ está relacionada con la contribución magnética de los átomos $X$, la cual va decreciendo conforme aumenta el $Z$, esto se debe a que los orbitales $3 d-X$ se van llenando, haciendo así más pequeña la diferencia entre las densidades up y down, los cuales están relacionados con el momento magnético del átomo $X$ $\left(m \equiv n_{\uparrow}(\mathbf{r})-n_{\downarrow}(\mathbf{r})\right)$. A tal punto que para el caso de $X=\mathrm{Cu}$ y Zn $(Z=29$ y 30), el valor de $M_{f u}$ se debe únicamente a la contribución magnética de los átomos FeII la cual no varía significantemente en todos estos compuestos $X \mathrm{Fe}_{3} \mathrm{~N}$ (Figura 3.7b).

Tabla 3.2: Valores del momento magnético por fórmula unidad $\left(M_{f u}\right)$ y de los momentos magnéticos por átomo de los compuestos ternarios $\mathrm{XFeN}$ obtenidos en el presente trabajo.

\begin{tabular}{ccccccc}
\hline$X$ & & Fe & Co & Ni & Cu & Zn \\
\hline$M_{f u}\left(\mu_{B} / f u\right)$ & & 9.71 & 8.94 & 8.06 & 7.01 & 5.73 \\
$m\left(\mu_{B}\right)$ & $X$ & 3.00 & 1.89 & 0.83 & 0.18 & 0.00 \\
& FeII & 2.21 & 2.33 & 2.34 & 2.24 & 2.11 \\
\hline
\end{tabular}




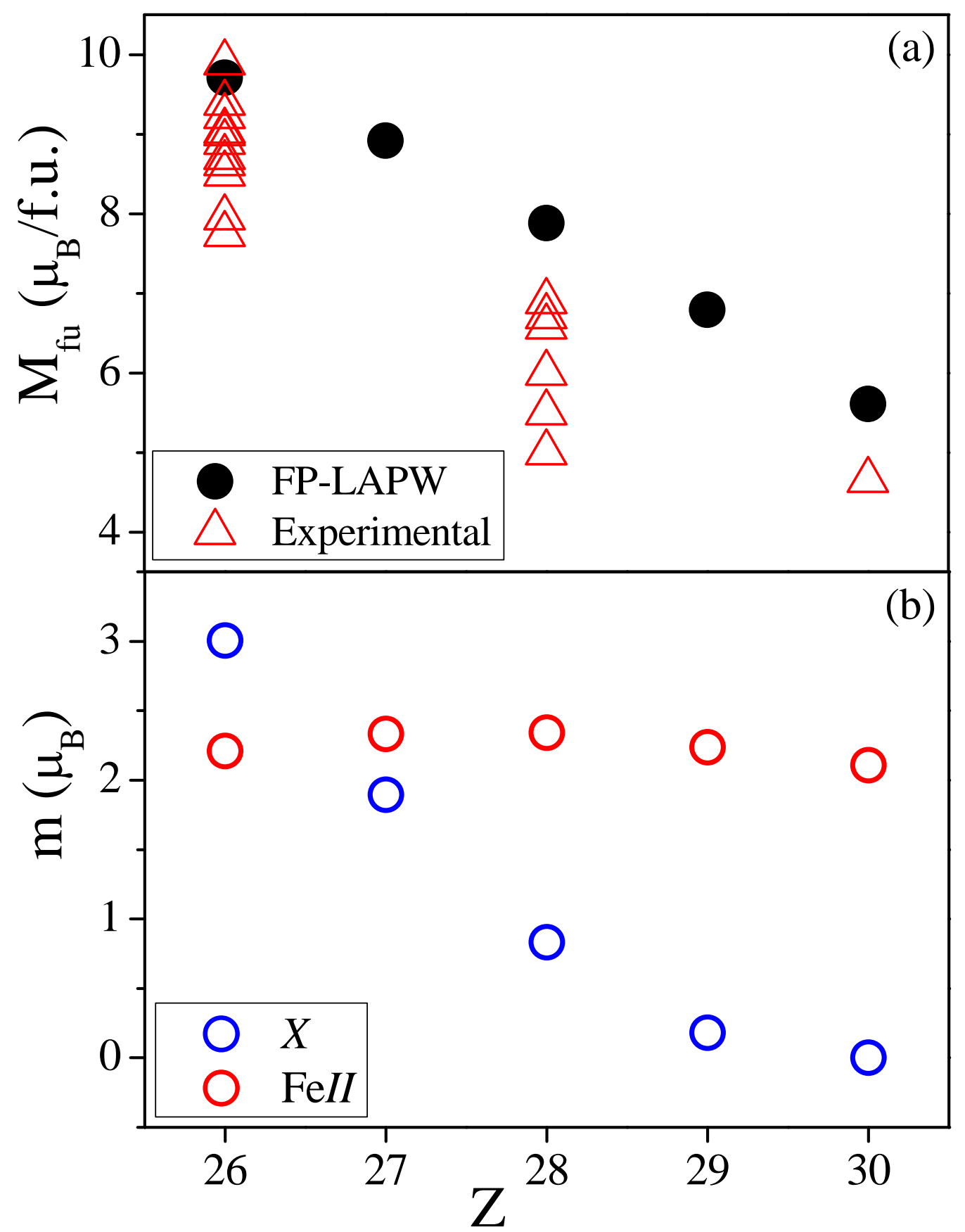

Figura 3.7: Variación respecto el $Z$ del átomo $X$ del a) $M_{f u} \mathrm{y}$ b) momento magnético de los átomos $\mathrm{X}$ y FeII de los compuestos ternarios $\mathrm{XFe}_{3} \mathrm{~N}$. 
En la Figura 3.8 se muestran las densidades de estados (DOS) de los compuestos $\mathrm{XFe}_{3} \mathrm{~N}$ respecto al nivel de Fermi $\left(E_{f}\right)$. Para el caso del $\mathrm{CoFe}_{3} \mathrm{~N}, \mathrm{NiFe}_{3} \mathrm{~N}$ y $\mathrm{CuFe}_{3} \mathrm{~N}$ es posible distinguir la existencia de una hibridización entre los orbitales $d$-FeII y $p$ - $\mathrm{N}$ en el rango de energía -9.0 eV y -5.0 eV mostrando así un fuerte enlace químico de carácter covalente entre dichos átomos, esta hibridización además está relacionada con el valor casi constante del momento magnético del átomo FeII. En el rango de energía -5.0eV y $E_{f}$ se encuentra una superposición de los estados electrónicos $d$-X y $d$-FeII con la presencia de una gran asimetría entre las DOS up y down, la cual da origen a los momentos magnéticos locales de los átomos X y FeII, además, para los átomos $X$ se observa un aumento en la población de estados down en regiones de energía menores a $E_{f}$ conforme aumenta el valor de $Z$ del átomo $X$, conllevando así a una disminución del momento magnético local del átomo $X$. Para el caso del $\mathrm{ZnFe}{ }_{3} \mathrm{~N}$, los estados electrónicos $d$-Zn se encuentran en el rango de energías de $-9.0 \mathrm{eV}$ a $-5.0 \mathrm{eV}$, hibridizándose con los orbitales $d$-FeII y $p$-N. Además, la ausencia de estados electrónicos $d-X$ up en $E_{f}$ indica un comportamiento ferromagnético fuerte de estos compuestos semejante al $\gamma^{\prime}-\mathrm{Fe}_{4} \mathrm{~N}$ [130]. 


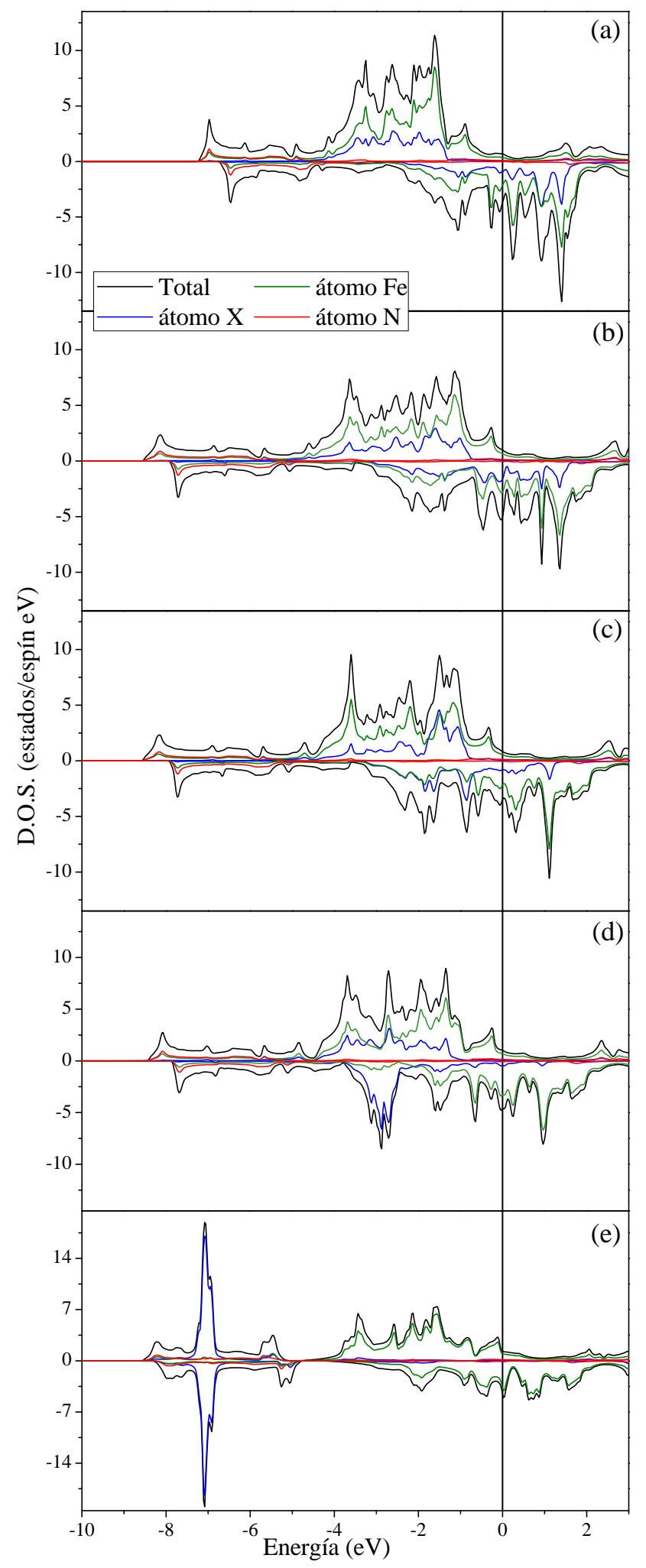

Figura 3.8: Densidad de estados del a) $\gamma^{\prime}-\mathrm{Fe}_{4} \mathrm{~N}$ y los compuestos ternarios b) $\mathrm{CoFe}_{3} \mathrm{~N}$, c) $\left.\mathrm{NiFe}_{3} \mathrm{~N}, \mathrm{~d}\right) \mathrm{CuFe}_{3} \mathrm{~N}$ y e) $\mathrm{ZnFe}{ }_{3} \mathrm{~N}$. 
El comportamiento decreciente lineal de $M_{f u}$ (Figura 3.7a) también puede ser analizado a través del modelo de Slater-Pauling-Friedel (SPF) [175], donde la curva de Slater-Pauling relaciona el momento magnético promedio del compuesto $(<m>)$ con la cantidad de electrones de valencia $\left(Z_{v}\right)$. Teniendo en cuenta que el momento magnético $(m)$ y la cantidad de electrones de valencia $\left(Z_{v}\right)$ cumplen las siguientes relaciones:

$$
\begin{aligned}
m & =n_{\uparrow}-n_{\downarrow} \\
Z_{v} & =n_{\uparrow}+n_{\downarrow}
\end{aligned}
$$

donde $n_{\uparrow}$ y $n_{\downarrow}$ representan las poblaciones de espín up y down, respectivamente. Combinando (3.1) y (3.2), se obtiene:

$$
\begin{aligned}
& m=2 n_{\uparrow}-Z_{v} \\
& m=Z_{v}-2 n_{\downarrow}
\end{aligned}
$$

Las expresiones (3.3) y (3.4), explican el comportamiento de las dos rectas de Slater con pendientes opuestas dependiendo que si se conoce $n_{\uparrow} \mathrm{o} n_{\downarrow}$, respectivamente.

Asumiendo que se conoce $n_{\uparrow}$ y que la contribución al momento magnético proviene mayormente de la polarización de las bandas $d$, se puede definir entonces la valencia magnética $\left(Z_{m}\right)[175,176]$ :

$$
Z_{m}=2 n_{d \uparrow}-Z_{v}
$$

J. Friedel [175], propuso que la cantidad de electrones $d$ cambia de forma discreta, de tal forma que $n_{d \uparrow}=0$ para los primero metales de transición como también para los elementos sin orbital $d$, mientras que para la segunda mitad de los metales de transición $n_{d \uparrow}=5$. Esto nos conduce a la curva de Slater-Pauling-Friedel (SPF), donde el valor de $\langle m>$ se representa como $[175,176]$ :

$$
<m>=Z_{m}+2 n_{s p \uparrow}
$$

donde $n_{s p \uparrow}$ es un valor constante de ajuste proveniente de la contribución de los orbitales s y $p$ al momento magnético. En la Figura 3.9, se muestra la variación del $<m>$ respecto del $Z_{v}$ para estos compuestos ternarios, observándose una buena 
correlación entre los valores obtenidos por los cálculos ab-initio, el modelo SPF y los datos experimentales obtenidos de la bibliografía. La sobrestimación del modelo de SPF podría estar relacionado con el hecho que en este modelo se propone que los momentos magnéticos atómicos están localizados y no se considera una contribución proveniente del intersticio. Pero a pesar que el modelo de SPF es un modelo simplificado del magnetismo, describe satisfactoriamente el comportamiento del momento magnético de los compuestos ternarios $\mathrm{XFe}_{3} \mathrm{~N}$. Este modelo también ha sido aplicado de forma satisfactoria a otros compuestos ferrosos [176, 178].

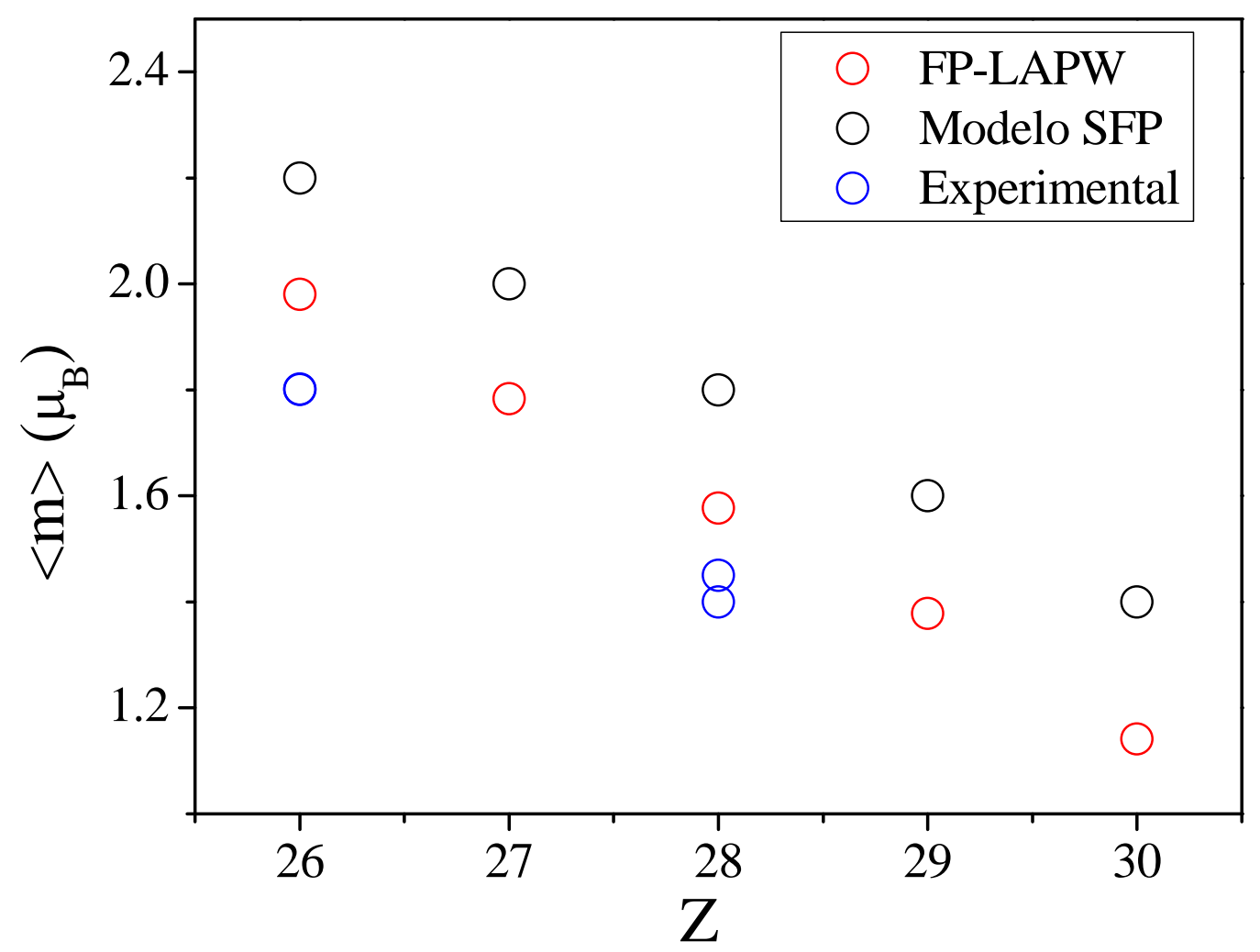

Figura 3.9: Dependencia del $<m>$ respecto $Z_{v}$ para los compuestos ternarios $X \mathrm{Fe}_{3} \mathrm{~N}$. 
Consecuentemente, al sustituir el átomo $\mathrm{FeI}$ por un átomo de $\mathrm{Co}, \mathrm{Ni}, \mathrm{Cu}$ o $\mathrm{Zn}$ en el compuesto $\gamma^{\prime}-\mathrm{Fe}_{4} \mathrm{~N}$ con el fin de obtener compuestos ternarios $\mathrm{XFe}_{3} \mathrm{~N}$ con propiedades magnéticas semejantes al $\gamma^{\prime}-\mathrm{Fe}_{4} \mathrm{~N}$, modifica también las propiedades hiperfinas de los átomos de Fe (FeII) que forman parte de estos materiales. Dichas propiedades hiperfinas están caracterizadas por el campo hiperfino $\left(B_{h f}\right)$, el corrimiento isomérico $(\delta)$ y el desdoblamiento cuadrupolar $(\varepsilon)$ del átomo de Fe (Tabla 3.3).

Tabla 3.3: Valores del momento magnético por fórmula unidad $\left(M_{f u}\right)$ y de los momentos magnéticos por átomo de los compuestos ternarios $\mathrm{XFeN}$ obtenidos en el presente trabajo.

\begin{tabular}{cccccc}
\hline$X$ & $\mathrm{Fe}$ & $\mathrm{Co}$ & $\mathrm{Ni}$ & $\mathrm{Cu}$ & $\mathrm{Zn}$ \\
\hline$B_{h f}(\mathrm{~T})$ & 24.08 & 21.68 & 19.66 & 18.04 & 17.64 \\
$\delta(\mathrm{mm} / \mathrm{s})$ & 0.30 & 0.30 & 0.31 & 0.36 & 0.40 \\
$\varepsilon(\mathrm{mm} / \mathrm{s})$ & 0.49 & 0.56 & 0.67 & 0.73 & 0.88 \\
\hline
\end{tabular}

En la Figura 3.10, se muestra la variación del $B_{h f}, \delta$ y $\varepsilon$ en función de $Z$ del átomo $X$ junto con los valores experimentales obtenidos de la bibliografía (Tabla 2.4), donde se puede observar que el $B_{h f}$ decrece linealmente conforme aumenta el valor de $Z$ (Figura 3.10a), con una pendiente de $-1.7 \mathrm{~T}$, mientras que los valores experimentales decrecen con una pendiente de $-1.2 \mathrm{~T}$, mostrando una buena correlación entre los valores calculados y los experimentales. Esta disminución de $B_{h f}$ está relacionada con una mayor población de los estados down cercanos al núcleo y/o a una disparidad de los electrones $3 d$-Fe los cuales polarizan a los electrones $s$-Fe. Los parámetros $\delta$ y $\varepsilon$, también varían linealmente con $Z$, pero de forma creciente. Por un lado, el aumento de $\delta$ con $Z$ del átomo $X$ indica una mayor densidad de carga cercana al núcleo del átomo Fe respecto al $\alpha$ - Fe (BCC) (Figura 3.10b), mientras que el incremento de $\varepsilon$ respecto al $Z$ del átomo $X$ está relacionado con el aumento de la asimetría esférica de la densidad de carga electrónica cercana al núcleo (Figura 3.10c). Las dependencias de $\delta$ y $\varepsilon$ con $Z$ obtenidas son corroboradas 
con los valores experimentales recopilados (Tabla 2.4).

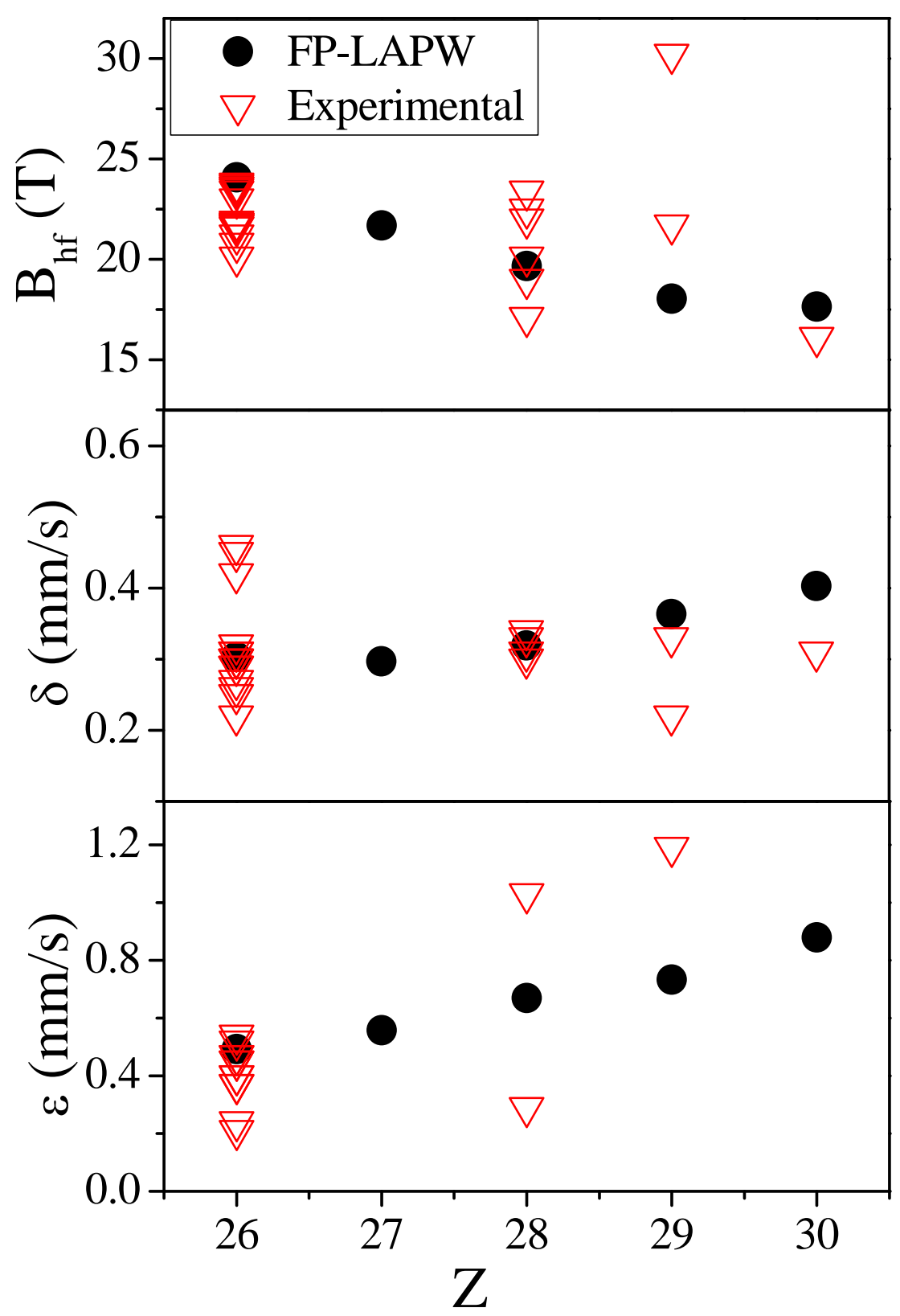

Figura 3.10: Valores del a) $B_{h f}$, b) $\delta$ y c) $\varepsilon$ del átomo FeII de los compuestos $\mathrm{XFe}_{3} \mathrm{~N}$, en función del Z del átomo $X$.

Ahora abordaremos el estudio del compuesto ternario $\mathrm{ScFe}_{3} \mathrm{~N}$. En la Figura 3.11, se muestra la variación de la energía total en función del parámetro de red del compuesto para la fase magnética (FM) y no magnética (NM) en las estructuras 
cristalinas $P m \overline{3} m$ y $P 4 / m m m$. Dichos datos de volumen y energía fueron ajustados con la ecuación de estado de Birch-Murnaghan (2.2) con el fin de obtener el valor del volumen y parámetro de red de equilibrio. Estos son reportados en la Tabla 3.4 junto con la variación de la energía total $(\Delta E)$ respecto del estado NM de la estructura cristalina $P m \overline{3} m$, observándose que, a diferencia de los otros compuestos ternarios descritos anteriormente con igual estructura cristalina, el $\mathrm{ScFe}_{3} \mathrm{~N}$ posee un estado fundamental no magnético.

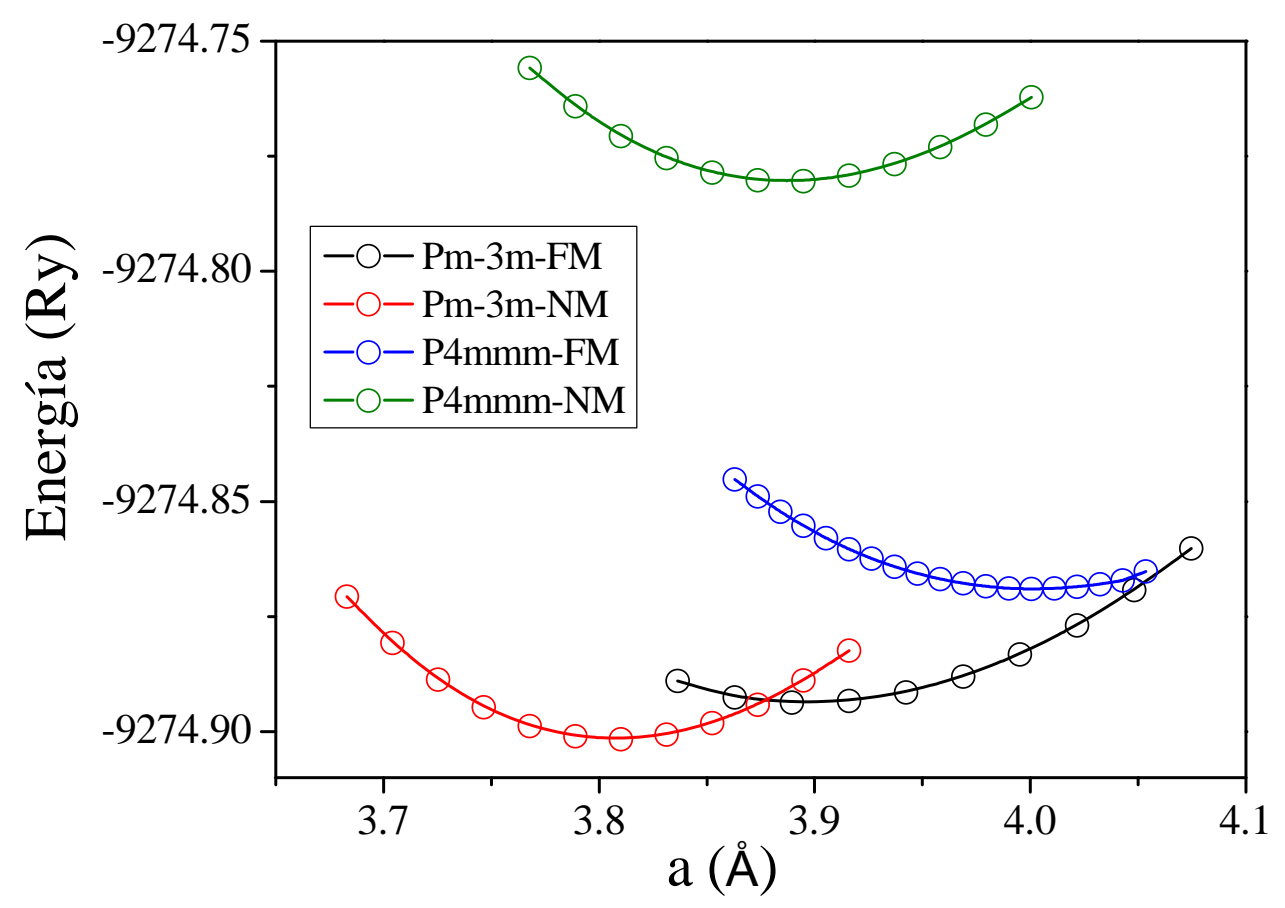

Figura 3.11: Curvas de energía total - parámetro de red del compuesto $\mathrm{ScFe}_{3} \mathrm{~N}$.

Tabla 3.4: Parámetro de red de equilibrio del $\mathrm{ScFe}_{3} \mathrm{~N}$, para las dos estructuras cristalinas en las fase magnética (FM) y no magnética (NM), junto con la variación de la energía total respecto del estado $P m \overline{3} m$ no magnético.

\begin{tabular}{ccccc}
\hline & $P m \overline{3} m(\mathrm{NM})$ & $P m \overline{3} m(\mathrm{FM})$ & $P 4 / m m m(\mathrm{NM})$ & $P 4 / m m m(\mathrm{FM})$ \\
\hline$a(\AA)$ & 3.8031 & 3.8959 & 3.8868 & 3.9973 \\
$\Delta E(\mathrm{mRy})$ & 0.0 & 7.9 & 121.1 & 31.5 \\
\hline
\end{tabular}


De la Figura 3.11 se puede observar la existencia de una posible transición de la fase NM a la FM bajo una expansión del compuesto $\mathrm{ScFe}_{3} \mathrm{~N}$ con estructura $\operatorname{Pm} \overline{3} m$. En la Figura 3.12 se muestra la entalpía de estas fases magnéticas en función de la presión, las cuales se interceptan en -3.86GPa. Indicando que el $\mathrm{ScFe}_{3} \mathrm{~N}$, bajo esta presión expansiva podría mostrar una fase magnética con un $M_{f u}$ de $5.41 \mu_{B}$, donde los momentos magnéticos del Sc y de los FeII se encuentran alineados antiparalelamente con valores de $1.94 \mu_{B}$ y $-0.24 \mu_{B}$, respectivamente.

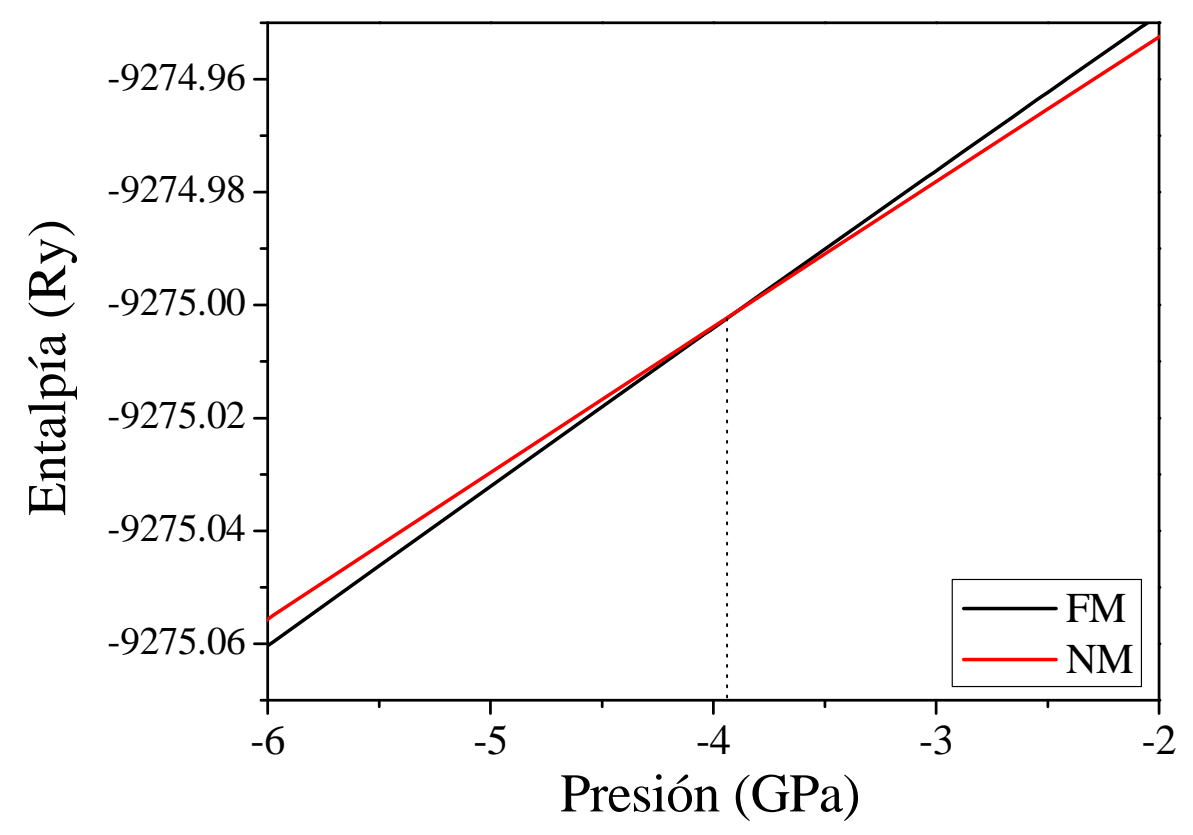

Figura 3.12: Intercepción de las curvas presión de la entalpía para los casos FM y NM del $\mathrm{ScFe}_{3} \mathrm{~N}$ con estructura $\operatorname{Pm} \overline{3} m$. 


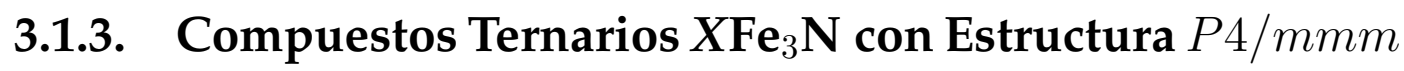

En la presente sección, se analizaran las propiedades estructurales, magnéticas e hiperfinas de los compuestos ternarios $X \mathrm{Fe}_{3} \mathrm{~N}$ donde el átomo $X(X=\mathrm{Ti}, \mathrm{V}, \mathrm{Cr} \mathrm{y}$ $\mathrm{Mn}$ ) sustituye a un átomo FeII, es decir, un átomo ubicado en una de las caras de la celda cúbica del $\gamma^{\prime}-\mathrm{Fe}_{4} \mathrm{~N}$. Esta sustitución preferencial del $X$ está relacionado con la afinidad química entre el átomo de $\mathrm{N}$ y el átomo $X$ la cual aumenta para átomos $3 d$ con menor número atómico que el Fe [133,151]. Además, se hará un análisis detallado del compuesto $\mathrm{MnFe}_{3} \mathrm{~N}$ con el fin de determinar su estado fundamental magnético.

\section{Propiedades Estructurales}

Estos materiales forman una estructura cristalina tetraédrica $(a=b \neq c)$ tipo $P 4 / \mathrm{mmm}$ (Figura 3.1b). En relación a ello, fue necesario encontrar el valor de $c / a$ óptimo para cada compuesto, calculando la energía total del compuesto $\mathrm{XFe}_{3} \mathrm{~N}$ mediante la variación de $a$ y $c$, obteniéndose una superficie $E=E(a, c)$. Por ejemplo, en la Figura 3.13 se muestran las superficies obtenidas para los compuestos $\mathrm{TiFe}_{3} \mathrm{~N}$ y $\mathrm{CrFe}_{3} \mathrm{~N}$, las cuales fueron interpoladas para encontrar el valor de $a$ y $c$ perteneciente al valor de mínima energía; el mismo procedimiento fue realizado para los compuestos $\mathrm{VFe}_{3} \mathrm{~N}$ y $\mathrm{MnFe}_{3} \mathrm{~N}$.

En la Figura 3.14a se muestran los valores de $c / a$ en función del $Z$ del átomo $X$ de estos compuestos ternarios. Ahí, se observa una dependencia del tipo exponencial decreciente mientras que en la Figura 3.14b se grafica el valor de $c / a$ en función del radio metálico $\left(r_{m e t}\right)$ del átomo $X$, observándose una dependencia lineal entre ellos. Es notoria la influencia del $r_{m e t}$ en la tetragonalización de estos compuestos ternarios. 


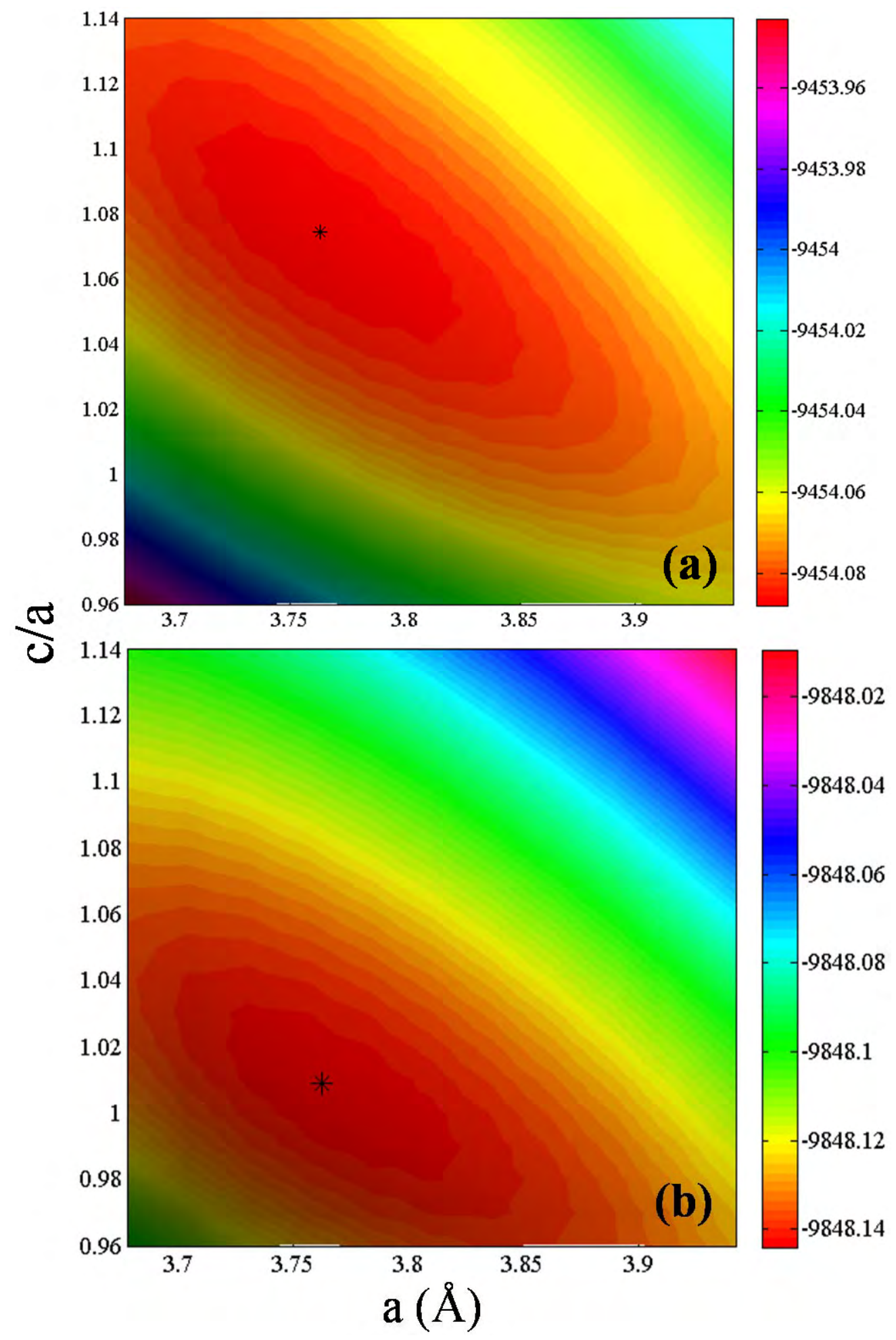

Figura 3.13: Superficie energética para los diferentes valores de $a$ y $c / a$ indicando el valor óptimo de $c / a$ para el caso de los compuestos ternarios a) $\mathrm{TiFe}_{3} \mathrm{~N}$ y b) $\mathrm{CrFe}_{3} \mathrm{~N}$. 

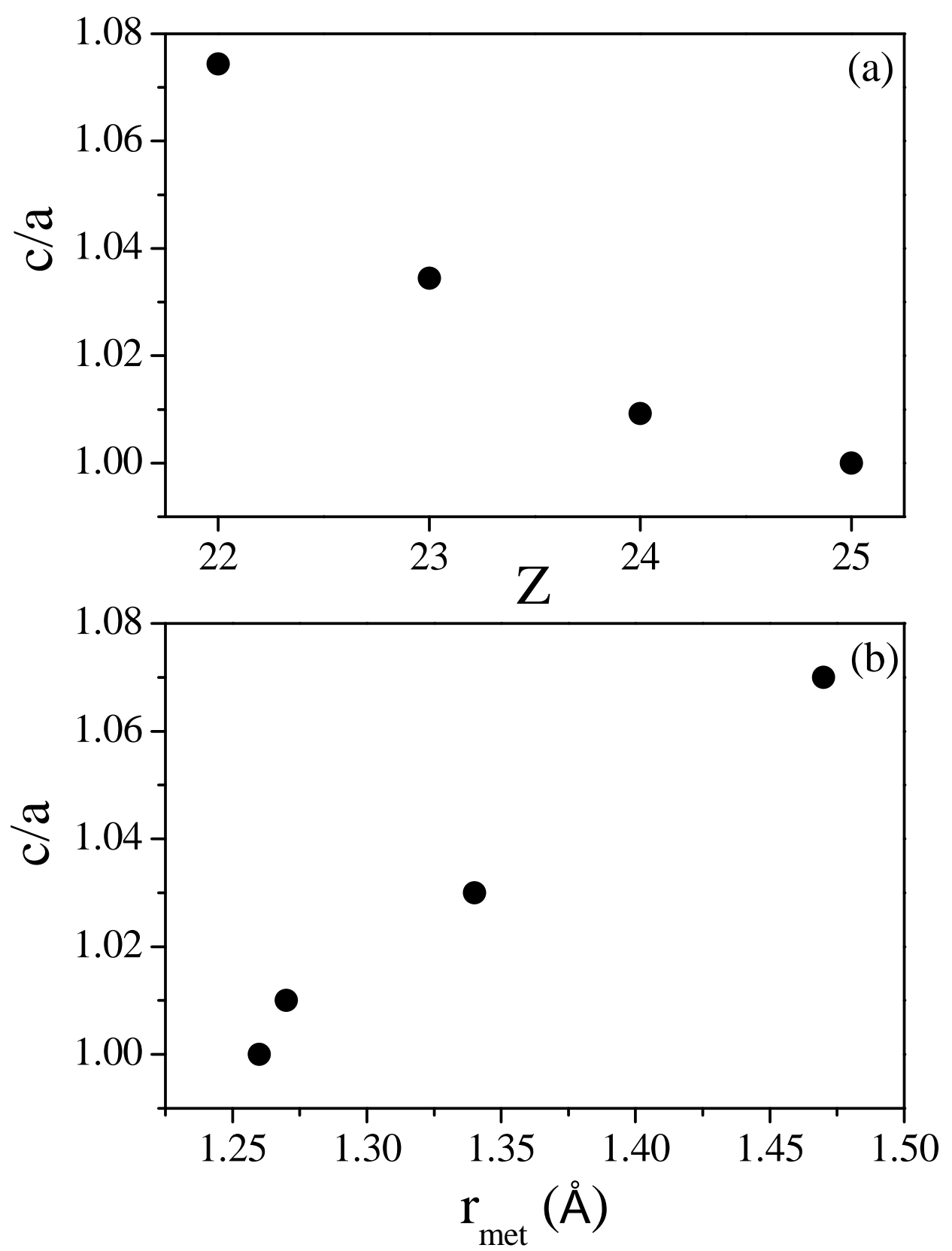

Figura 3.14: Dependencia de $c / a$ respecto del a) $Z$ y b) $r_{\text {met }}$ del átomo $X$ para los compuestos ternarios $\mathrm{XFe}_{3} \mathrm{~N}$. 
De forma complementaria, la optimización de $c / a$ se realizó también con el método de pseudopotenciales y ondas planas, código Quantum ESPRESSO [179], donde se realizó cálculos de relajación con celda variable, permitiéndonos optimizar todos los parámetros internos de la celda cristalina. Las optimizaciones se realizaron para cada compuesto ternario partiendo de la configuración cúbica de estos, es decir $c / a=1$, donde el criterio de convergencia usado fue la convergencia el tensor de Pulay hasta un valor de 1kbar. Ambos métodos brindan valores de $c / a$ que coinciden satisfactoriamente.

Tabla 3.5: Volumen $\left(V_{0}\right)$, parámetros de red $(a$ y $c$ ) de equilibrio y módulo de bulk $(B)$ de los compuestos ternarios $\mathrm{XFe}_{3} \mathrm{~N}$.

\begin{tabular}{ccccc}
\hline & $\mathrm{Ti}$ & $\mathrm{V}$ & $\mathrm{Cr}$ & $\mathrm{Mn}$ \\
\hline$V_{0}\left(\AA^{3}\right)$ & 57.377 & 54.222 & 53.900 & 54.453 \\
$c / a$ & 1.07 & 1.03 & 1.01 & 1.00 \\
$a(\AA)$ & 3.7658 & 3.7425 & 3.7690 & 3.7903 \\
$B(\mathrm{GPa})$ & 164.7 & 180.8 & 173.3 & 162.8 \\
\hline
\end{tabular}

Con los valores de $c / a$ óptimos de cada compuesto, se calculó la energía total para diferentes volúmenes usando el $c / a$ obtenido por el método FP-LAPW (Figura 3.15). Dichos datos fueron ajustados con la ecuación de estado de Birch-Murnaghan (2.2), permitiéndonos determinar el volumen $\left(V_{0}\right)$ y los parámetros de red de equilibrio ( $a$ y $c$ ) y el módulo de bulk $(B)$ de cada compuesto, los cuales se reportan en la Tabla 3.5. Estos valores difieren de otros valores teóricos existentes en la bibliografía (Tabla 2.5) debido a que éstos últimos fueron obtenidos considerando que el átomo $X$ sustituye al FeI. 


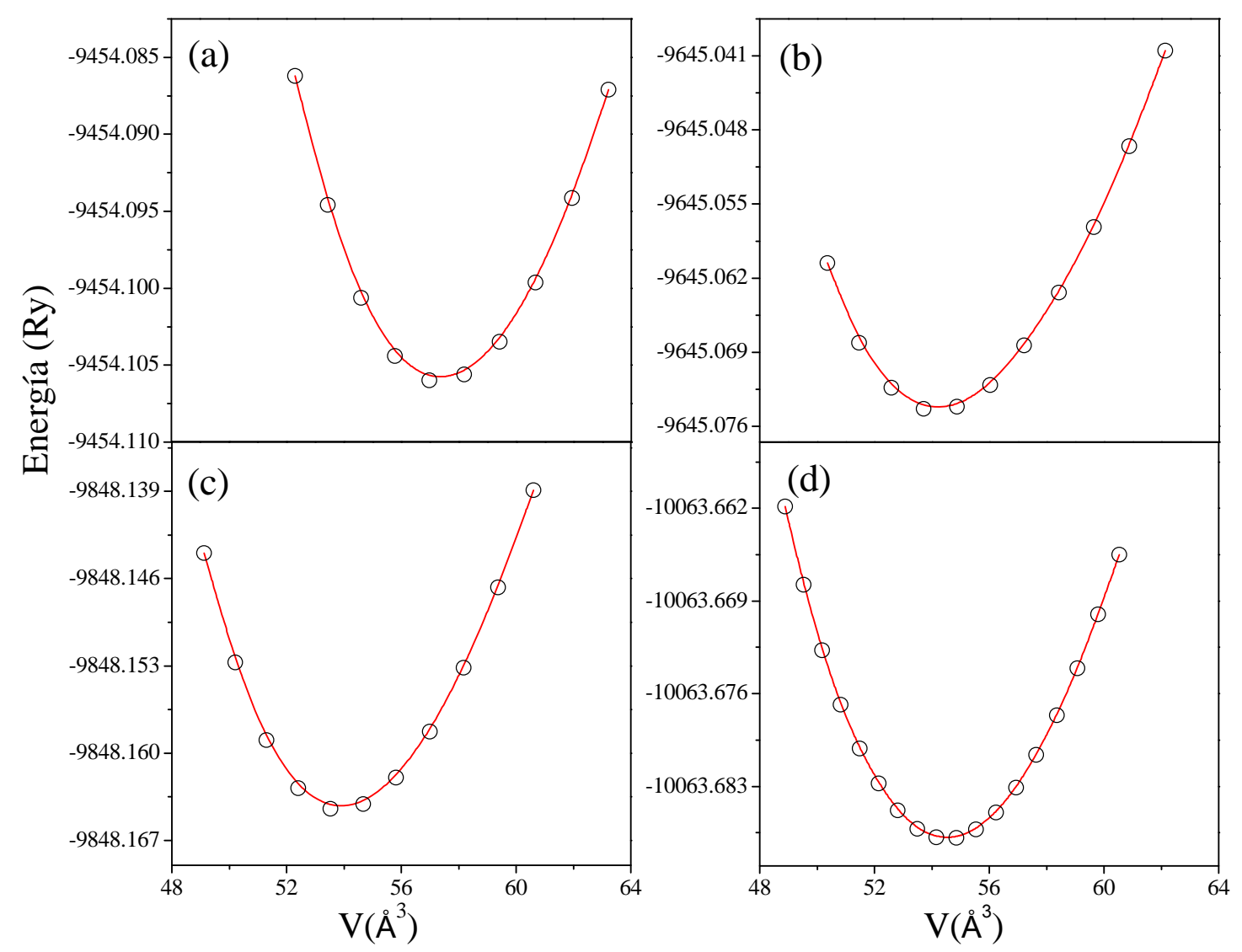

Figura 3.15: Curva energía - volumen para los compuestos ternarios a) $\left.\operatorname{TiFe}_{3} \mathrm{~N}, \mathrm{~b}\right) \mathrm{VFe}_{3} \mathrm{~N}$, c) $\mathrm{CrFe}_{3} \mathrm{~N}$ y d) $\mathrm{MnFe}_{3} \mathrm{~N}$. 


\section{Propiedades Magnéticas e Hiperfinas}

La sustitución de un átomo FeII por un átomo $X$ modifica también las propiedades magnéticas e hiperfinas de los compuestos ternarios $X \mathrm{Fe}_{3} \mathrm{~N}$, los cuales se reportan en la Tabla 3.6. En la figura 3.16a se muestra el valor de $M_{f u}$ en función del $Z$ del átomo $X$, observándose un decrecimiento casi lineal del $M_{f u}$ hasta un valor mínimo para el $\mathrm{CrFe}_{3} \mathrm{~N}$, mostrando luego un leve incremento para el $\mathrm{MnFe}_{3} \mathrm{~N}$ seguido de un aumento abrupto entre el $\mathrm{MnFe}_{3} \mathrm{~N}$ y el $\gamma^{\prime}-\mathrm{Fe}_{4} \mathrm{~N}$, de tal manera que el $M_{f u}$ del $\mathrm{TiFe}_{3} \mathrm{~N}$ es aproximadamente el $40 \%$ que del $\gamma^{\prime}-\mathrm{Fe}_{4} \mathrm{~N}$. Este comportamiento de $M_{f u}$ se debe al acoplamiento magnético antiparalelo existente entre los átomos de Fe y el átomo $X$. En la Figura 3.16b se observa que el momento magnético del átomo $X$ se incrementa conforme aumenta $Z$, con un valor máximo para $Z=25$ $\left(\mathrm{MnFe}_{3} \mathrm{~N}\right)$, mientras que el momento magnético del átomo FeII presenta una leve variación respecto a $Z$, esto no ocurre con el momento magnético del FeI. El incremento abrupto de $M_{f u}$ entre $Z=25\left(\mathrm{MnFe}_{3} \mathrm{~N}\right)$ y $Z=26\left(\gamma^{\prime}-\mathrm{Fe}_{4} \mathrm{~N}\right)$ está relacionado con un cambio en el tipo de acoplamiento magnético entre sus átomos, el cual es ferromagnético para el $\gamma^{\prime}-\mathrm{Fe}_{4} \mathrm{~N}$. Estos compuestos ternarios con estructura cristalina $P 4 / m m m$, debido al carácter magnético que presentan, se les puede caracterizar como compuestos ferrimagnéticos.

Tabla 3.6: Momento magnético por fórmula unidad $\left(M_{f u}\right)$ y momentos magnéticos por átomo de los compuestos ternarios $\mathrm{XFe}_{3} \mathrm{~N}$ con estructura $\mathrm{P} 4 / \mathrm{mmm}$.

\begin{tabular}{clccccc}
\hline$X$ & & $\mathrm{Ti}$ & $\mathrm{V}$ & $\mathrm{Cr}$ & $\mathrm{Mn}$ & $\mathrm{Fe}$ \\
\hline$M_{f u}\left(\mu_{B} / f u\right)$ & & 5.71 & 4.26 & 3.92 & 4.34 & 9.71 \\
$m\left(\mu_{B}\right)$ & $\mathrm{X}$ & -0.38 & -0.88 & -1.80 & -2.26 & 2.21 \\
& FeI & 2.33 & 2.40 & 2.51 & 2.53 & 3.00 \\
& FeII & 1.89 & 1.41 & 1.65 & 2.00 & 2.21 \\
\hline
\end{tabular}




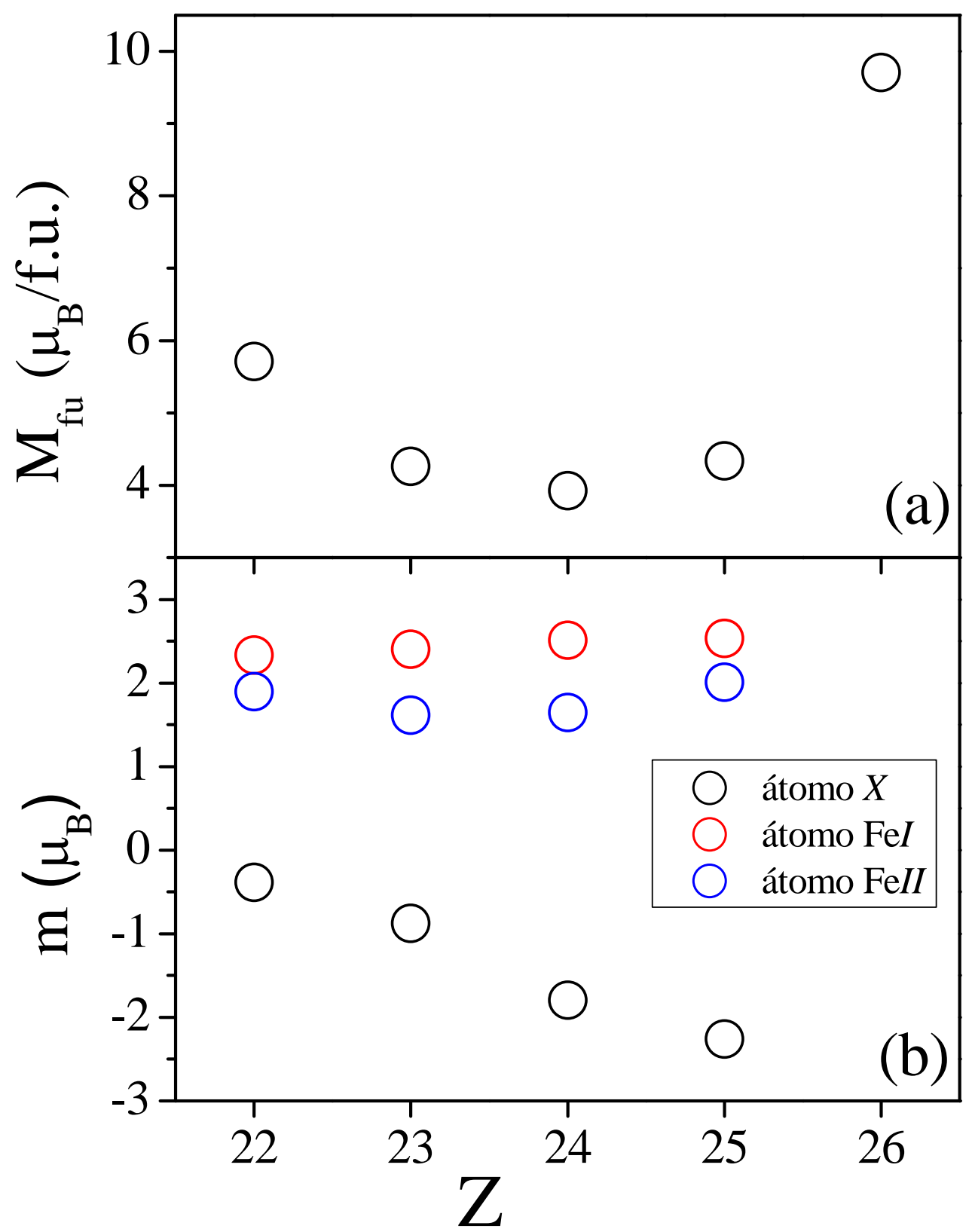

Figura 3.16: Variación del a) $M_{f u}$ y b) momento magnético por átomo en función del $Z$ del átomo $X$ de los compuestos ternarios $X \mathrm{Fe}_{3} \mathrm{~N}$ con estructura $P 4 / \mathrm{mmm}$. 
Una descripción complementaria del carácter magnético de estos compuestos se encuentra en el análisis de sus respectivas densidades de estados (DOS). En la Figura 3.17 se muestran las DOS de cada compuesto ternario con estructura $P 4 / m m m$, observándose tan solo poblados a los estados down de los orbitales $d$ $X$, los cuales se encuentran hibridizados con los estados $d$-Fe $I$ y $d$-FeII en el rango de energía de $-5.0 \mathrm{eV}$ y $E_{f}$. Esto muestra el tipo de acoplamiento antiferromagnético existente entre los átomos $X$ y los $\mathrm{Fe}$, mientras que en el rango de energías de $-8.5 \mathrm{eV}$ a $-5.0 \mathrm{eV}$ se encuentran hibridizados los orbitales $d$-FeII y $p-\mathrm{N}$, semejantes a los compuestos con estructura $\operatorname{Pm} \overline{3} m$. Además, se observa que la distribución de las densidades de estados pertenecientes al FeII no varía en todos estos compuestos. 


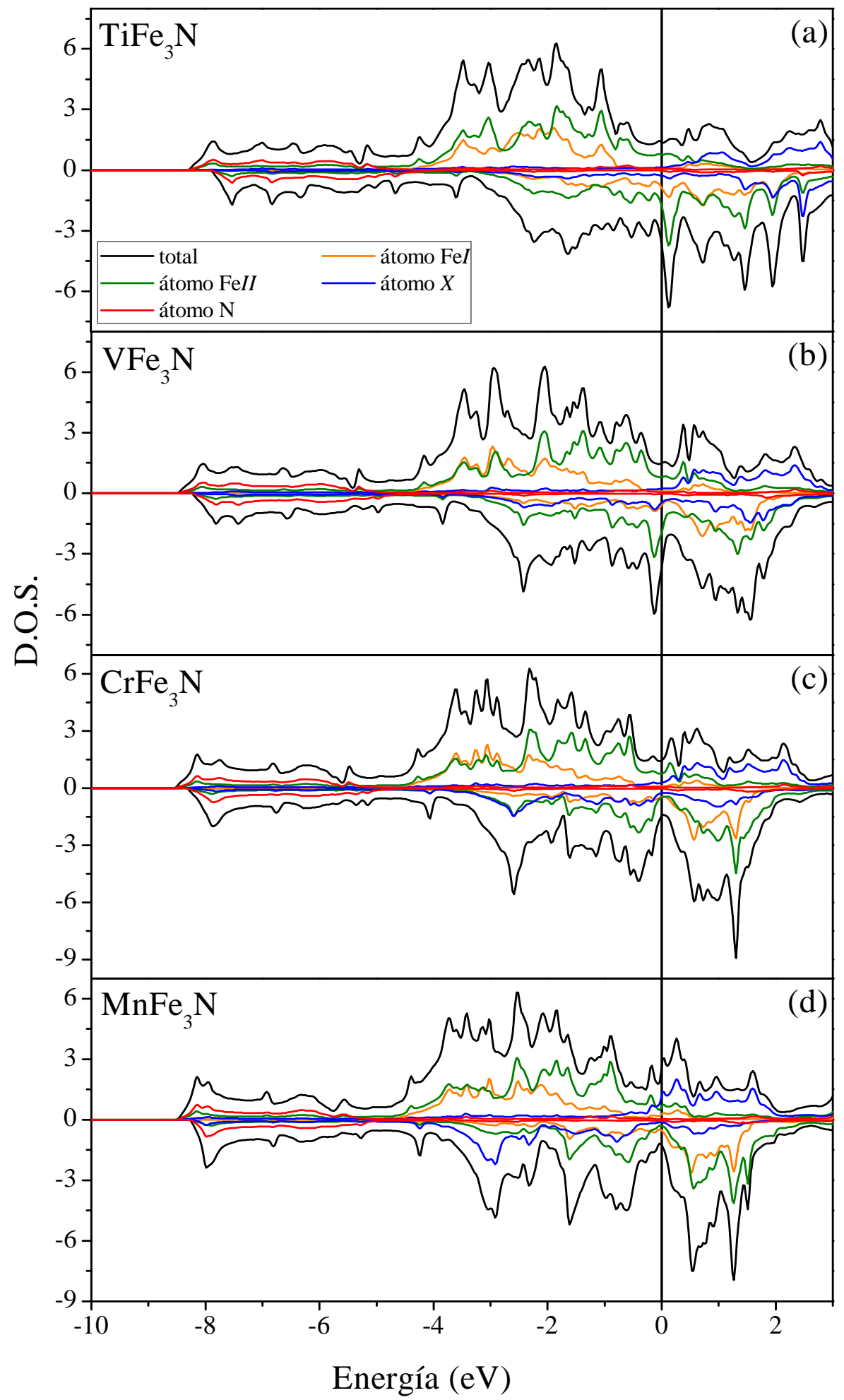

Figura 3.17: Variación del a) $M_{f u}$ y b) momento magnético por átomo en función del $Z$ del átomo $\mathrm{X}$ de los compuestos ternarios $\mathrm{XFe}_{3} \mathrm{~N}$ con estructura $P 4 / \mathrm{mmm}$. 
El comportamiento magnético de estos compuestos también cumple con la regla de acoplamiento magnético propuesta por J. M. D. Coey [180] en donde postula que entre átomos cuyo orbital $d$ está ocupado más de la mitad y átomos con el orbital $d$ ocupado menos de la mitad, existe un acoplamiento antiferromagnético. Además, usando el modelo de SPF [175] se obtiene una buena descripción del comportamiento magnético de estos compuestos. En la figura 3.18 se observa el valor de $<m>$ en función del $Z_{v}$ obtenido de los cálculos ab-initio y el modelo SPF, apreciándose una buena correlación entre dichos modelos, los cuales adicionalmente describen el cambio abrupto del comportamiento magnético entre el $\mathrm{MnFe}_{3} \mathrm{~N}$ y el $\gamma^{\prime}-\mathrm{Fe}_{4} \mathrm{~N}$.

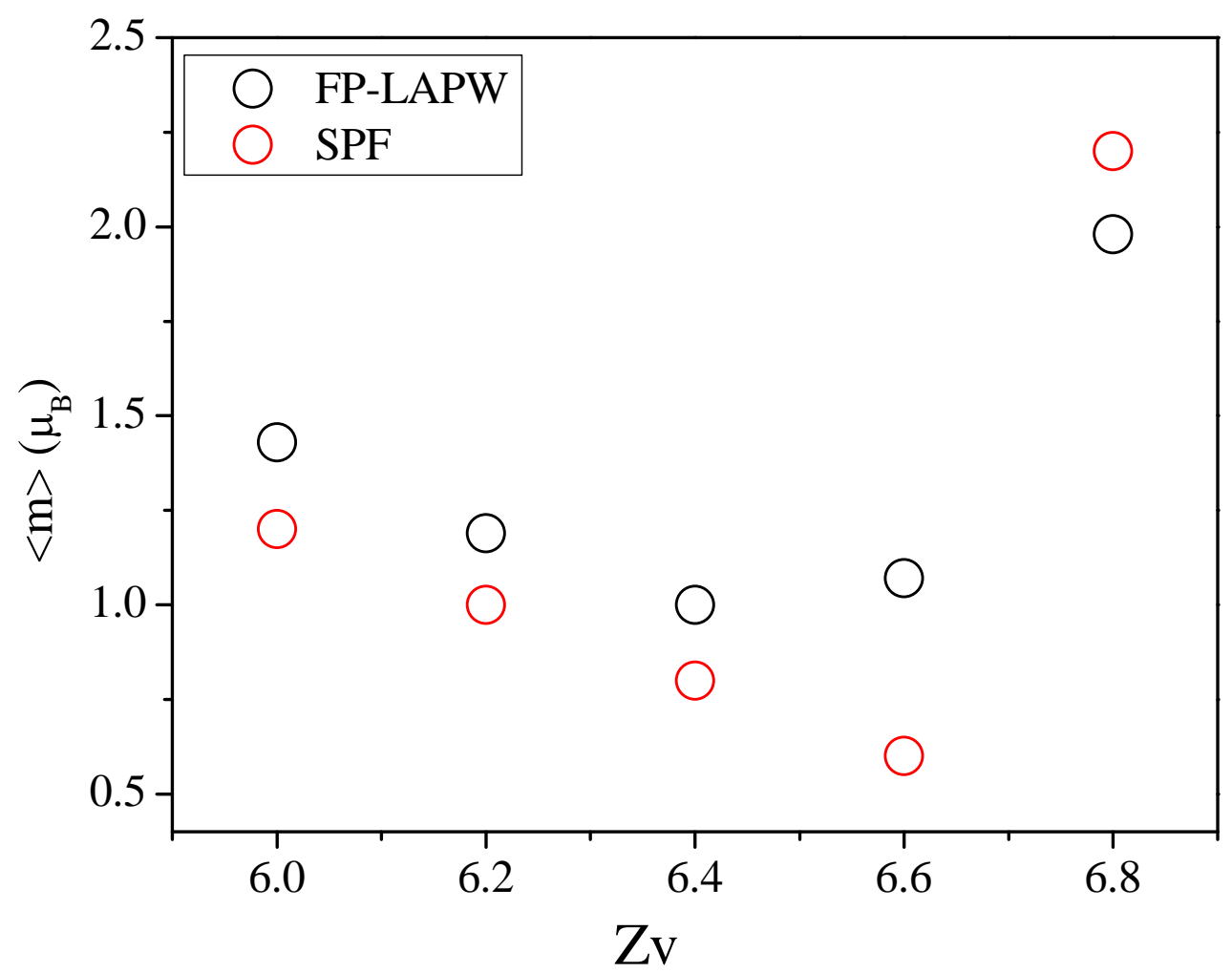

Figura 3.18: Valores de $<m>$ en función de $Z_{v}$ obtenido por los cálculos ab-initio y con el modelo SPF. 
En la Figura 3.19 (a, b y c) se muestran los valores del $B_{h f}, \delta$ y $\varepsilon$ de los átomos de Fe, FeI y FeII, en función del número atómico de $X(Z)$, observándose una disminución gradual del $B_{h f}$ (Figura 3.19a) con un valor mínimo en el compuesto $\mathrm{CrFe}_{3} \mathrm{~N}$, en donde los átomos $\mathrm{Fe} I$ y FeII poseen el mismo valor de $B_{h} f$, para luego incrementar conforme aumenta el valor de Z. Este comportamiento descrito está relacionado con la polarización negativa de los estados electrónicos cercanos al núcleo $[160,161]$ y a la interacción antiferromagnética existente entre el átomo $X$ y los Fe. El corrimiento isomérico (Figura 3.19b) de los átomos FeII no varía para todos los compuestos ternarios, mientras que para el FeI se observa una mayor influencia por el hecho de tener al átomo $X$ como primer vecino, modificando así la distribución de carga electrónica cercana del núcleo de los átomos FeI. Esto se ve reflejado además en el desdoblamiento cuadrupolar de los átomos FeI y FeII (Figura 3.19c), especialmente en los FeII en donde se observa un decrecimiento de $\varepsilon$ conforme aumenta $Z$, indicando una menor asimetría esférica de la distribución de carga electrónica cercana al núcleo. Luego en $Z=25$, cuyo orbital $3 d-X$ está ocupado la mitad, se observa un cambio de signo del $\varepsilon$, representando un cambio abrupto de la distribución de carga electrónica, esto es, de una configuración tipo esferoide prolato a una tipo esferoide oblato. El cambio de signo de $\varepsilon$ está también relacionado con el ángulo que forma el eje de magnetización y la dirección del gradiente de campo eléctrico de los átomos de Fe, donde el signo positivo indica un alineamiento paralelo mientras que el signo negativo indica un alineamiento antiparalelo. 


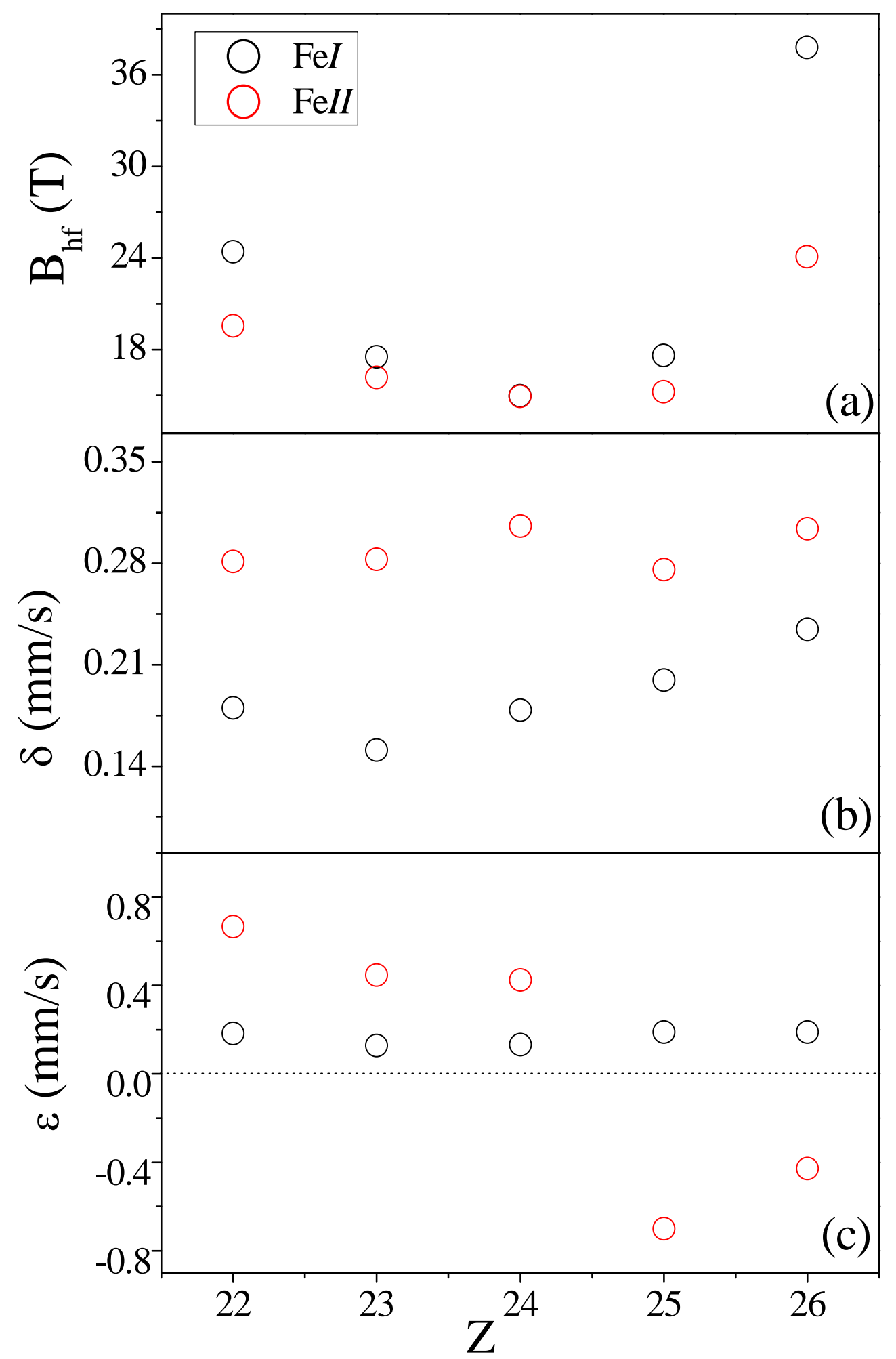

Figura 3.19: Variación del a) $B_{h f}$, b) $\delta$ y c) $\varepsilon$ en función del $Z$ del átomo $X$ para los compuestos ternarios $\mathrm{XFe}_{3} \mathrm{~N}$ con estructura $P 4 / \mathrm{mmm}$. 


\section{Estado Fundamental del $\mathrm{MnFe}_{3} \mathrm{~N}$}

El compuesto ternario $\mathrm{MnFe}_{3} \mathrm{~N}$ ha sido propuesto como una alternativa al $\gamma^{\prime}$ $\mathrm{Fe}_{4} \mathrm{~N}$, pero existe muy poca información experimental sobre dicho material [12]. En [12] no fue posible distinguir el sitio preferencial de sustitución del Mn en el $\gamma^{\prime}-\mathrm{Fe}_{4} \mathrm{~N}$, llegando a la conclusión de que el Mn puede sustituir de forma no preferencial tanto a un átomo FeI o un FeII. Desde el punto de vista teórico usando diferente métodos ab-initio siempre han considerado que el Mn sustituye al FeI debido a la similitud de los radios atómicos entre el Fe y Mn, llegando así a distintos estados fundamentales magnéticos [125, 127, 128]. Solo en [126] consideraron que el Mn podría sustituir a un átomo FeII formando la estructura cristalina $P 4 / \mathrm{mmm}$, donde los momentos magnéticos de los átomos de Mn y Fe son paralelos.

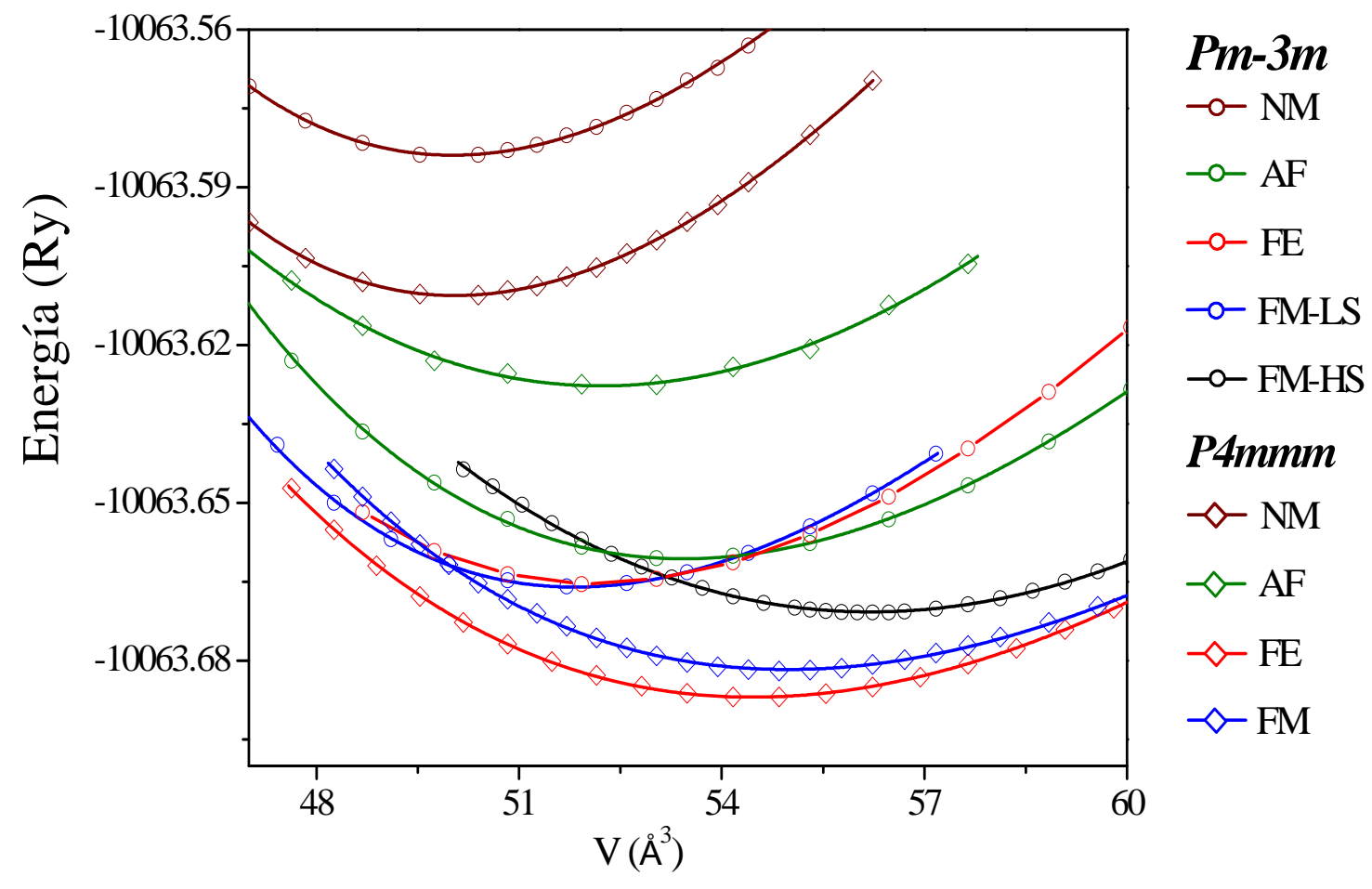

Figura 3.20: Curvas energía total - volumen para el compuesto $\mathrm{MnFe}_{3} \mathrm{~N}$ en las estructuras cristalinas $P m \overline{3} m$ y $P 4 / m m m$ y en las configuraciones magnéticas NM, AF, FE y FM.

En la presente sección se estudiará el estado fundamental del $\mathrm{MnFe}_{3} \mathrm{~N}$, comparando las energías totales cuando el Mn sustituye a un FeI o a un FeII, además de 
considerar los estados magnéticos: no magnético (NM), antiferromagnético (AF), ferromagnético (FM) y ferrimagnético (FE) de dicho compuesto.En la Figura 3.20 se muestra la variación de la energía total en función del volumen para las estructuras $P m \overline{3} m$ y $P 4 / m m m$, para las configuraciones NM, FM y FE. En la estructura $\operatorname{Pr} \overline{3} m$ el estado ferromagnético posee un estado de high spin (HS) y low spin (LS). En la Tabla 3.7 se muestran los valores del parámetro de red de equilibrio $(a)$, el módulo de bulk $(B)$ y las diferencias de energías $(\Delta E)$ respecto al estado $P 4 / m m m$ ferrimagnético, donde es posible distinguir que las demás configuraciones se encuentran a mayor energía. Esto nos indica que el Mn prefiere sustituir a un FeII formando la estructura cristalina $P 4 / \mathrm{mmm}$ con un parámetro de red semejante al del $\gamma^{\prime}-\mathrm{Fe}_{4} \mathrm{~N}$, donde los momentos magnéticos de los átomos de Fe y Mn están alineados antiparalelamente con un momento magnético resultante distinto a cero, esto es, el compuesto $\mathrm{MnFe}_{3} \mathrm{~N}$ posee un estado fundamental $\mathrm{P} 4 / \mathrm{mmm}$ ferrimagnético. Este resultado difiere de otros estudios teóricos en donde sólo consideraron al compuesto ternario $\mathrm{MnFe}_{3} \mathrm{~N}$ en la fase cristalina $P m \overline{3} m$ obteniendo diferentes estados fundamentales magnéticos tales como ferromagnéticos o ferrimagnético [125, 127, 128]. En [126] usando el método FP-LASTO consideraron al $\mathrm{MnFe}_{3} \mathrm{~N}$ con la estructura cristalina $P 4 / \mathrm{mmm}$, determinando que el estado fundamental magnético es FM.

También en la Tabla 3.7 se puede observar que todas las configuraciones magnéticas (NM, FE y FM) pertenecientes a la fase cristalina $P 4 / m m m$ se encuentran a menores energías que la fase cristalina $\operatorname{P} m \overline{3} m$, mientras que para la configuración magnética AF sucede lo contrario. Con el fin de analizar la estabilidad magnética del compuesto ternario $\mathrm{MnFe}_{3} \mathrm{~N}$ se realizaron cálculos fixed spin moment (FSM, momento magnético fijo), donde se fijó el valor de $M_{f u}$ desde $0 \mu_{B}$ hasta $12 \mu_{B}$, y en cada valor de $M_{f u}$ se optimizó el parámetro de red del compuesto.

En la Figura 3.21 se muestra la dependencia con $M_{f u}$ de la variación de la energía total del $\mathrm{MnFe}_{3} \mathrm{~N}$ respecto del estado magnético $\mathrm{AF}\left(\Delta E=E\left(M_{f u}\right)\right.$ $E(0))$, tanto para la fase cristalina $P m \overline{3} m$ y $P 4 / m m m$, calculados en sus respectivos parámetros de red de equilibrio de cada valor de $M_{f u}$. Para la fase $P m \overline{3} m$ las 
Tabla 3.7: Parámetro de red de equilibrio $(a)$, módulo de bulk $(B)$ y variación de la energía respecto del estado $P 4 / \mathrm{mmm}$-FE.

\begin{tabular}{ccccc}
\hline & & $a(\AA)$ & $B(\mathrm{GPa})$ & $\Delta \mathrm{E}(\mathrm{meV})$ \\
\hline$P m \overline{3} m$ & NM & 3.6843 & 281.8 & 1400.0 \\
& AF & 3.7666 & 213.8 & 362.6 \\
& FM(LS) & 3.7282 & 250.2 & 287.8 \\
& FM(HS) & 3.8304 & 171.5 & 219.1 \\
& FE & 3.7273 & 236.9 & 303.1 \\
& NM & 3.6852 & 291.3 & 1038.6 \\
& AF & 3.7377 & 198.0 & 806.5 \\
& FM & 3.8023 & 158.0 & 69.0 \\
& FE & 3.7903 & 162.7 & 0.0 \\
\hline
\end{tabular}

configuraciones magnéticas FE y FM-LS pertenecen a mínimos locales respecto a la configuración FM-HS, con la existencia de una barrera de energía de $12.4 \mathrm{meV}$ entre el estado magnético FE y FM-LS y de una barrera de $37.1 \mathrm{meV}$ del estado magnético FM-LS a FE. Mientras que la barrera de energía entre el estado FM-LS a FM-HS es de $157.4 \mathrm{meV}$ y de $193.6 \mathrm{meV}$ en el sentido inverso. Para el caso de la fase $P 4 / \mathrm{mmm}$, se observa una barrera de energía entre el estado FE y FM de $212.4 \mathrm{meV}$ y de $143.4 \mathrm{meV}$ en el sentido inverso, siendo el estado magnético FE un mínimo global. 


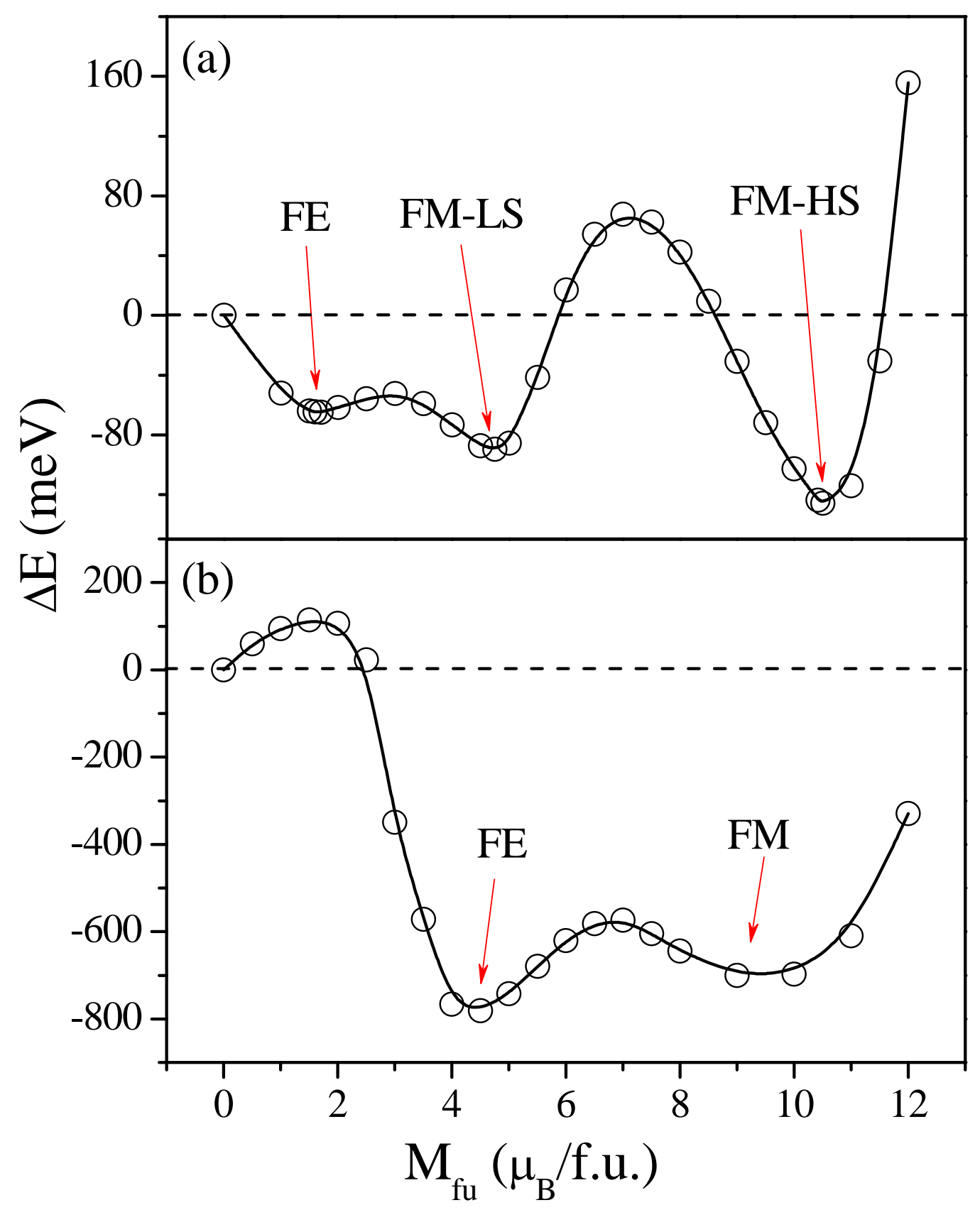

Figura 3.21: Variación de la energía total, respecto del estado AF, en función del $M_{f u}$ del compuesto $\mathrm{MnFe}_{3} \mathrm{~N}$ para las estructuras cristalinas a) $P m \overline{3} m$ y b) $P 4 / m m m$. 
La barrera de energía entre el estado FE y FM para la estructura cristalina $P 4 / m m m$ (Figura 3.21b), indica una posible transición de estado magnético bajo una pequeña variación de volumen del $\mathrm{MnFe}_{3} \mathrm{~N}$ debido a la proximidad del parámetro de red existente entre ambas configuraciones magnéticas (Tabla 3.7), esto es conocido como el efecto magneto-volumétrico [181]. O a través de un cambio térmico que permita vencer la correspondiente barrera de energía, conduciendo a una transición de estado magnético. La importancia de esta transición de fase magnética radica en el aumento de un $120 \%$ en la magnetización del $\mathrm{MnFe}_{3} \mathrm{~N}$.

Finalmente, en la Tabla 3.8 se reportan los valores de los momentos magnéticos totales $\left(M_{f u}\right)$, por átomo y los campos hiperfinos de los átomos de Fe de este compuesto, para los estados magnéticos considerados en ambas estructuras cristalinas.

Tabla 3.8: Valores del $M_{f u}$, momento magnético por átomo y campo hiperfino $\left(B_{h f}\right)$ de los átomos de $\mathrm{Fe}$ del compuesto $\mathrm{MnFe}_{3} \mathrm{~N}$, para las estructuras cristalinas $P m \overline{3} m$ y $P 4 / m m m$ en las fases magnéticas AF, FE y FM.

\begin{tabular}{lccccccc}
\hline & \multicolumn{3}{c}{$P m \overline{3} m$} & \multicolumn{3}{c}{$P 4 / m m m$} \\
& AF & FE & FM-LS & FM-HS & AF & FE & FM \\
\hline$M_{f u}\left(\mu_{B} / f u\right)$ & 0.00 & 1.70 & 4.75 & 10.68 & 0.00 & 4.34 & 9.55 \\
$m_{\mathrm{Mn}}\left(\mu_{B}\right)$ & -3.79 & -3.26 & 3.36 & 3.64 & -2.24 & -2.26 & 2.07 \\
$m_{\mathrm{FeI}}\left(\mu_{B}\right)$ & 1.28 & 0.70 & 0.45 & 2.25 & 0.40 & 2.53 & 2.85 \\
$m_{\mathrm{FeII}}\left(\mu_{B}\right)$ & - & - & - & - & 0.98 & 2.00 & 2.15 \\
$B_{h f-\mathrm{FeI}}(\mathrm{T})$ & 5.93 & 2.61 & 12.20 & 24.52 & 0.11 & 17.62 & 36.82 \\
$B_{h f-\mathrm{FeII}}(\mathrm{T})$ & - & - & - & - & 5.88 & 15.25 & 27.66 \\
\hline
\end{tabular}




\subsection{Compuestos Ternarios $X F e_{3} N(X$ metal $4 d)$}

En la presente sección, inicialmente, se estudiará cuál es el sitio preferencial de sustitución de un metal $4 d(X=\mathrm{Ru}, \mathrm{Rh}, \mathrm{Pd}$ o $\mathrm{Ag})$ en el compuesto $\gamma^{\prime}-\mathrm{Fe}_{4} \mathrm{~N}$, para luego estudiar la variación las propiedades estructurales, magnéticas e hiperfinas de estos compuestos ternarios formados.

\subsubsection{Sitio de Sustitución Preferencial del Átomo $X$}

Con un procedimiento semejante al utilizado para el estudio de los compuestos ternarios $X \mathrm{Fe}_{3} \mathrm{~N}$ cuando $X$ es un metal $3 d$, se determinó el volumen de equilibrio tanto para la estructura $P m \overline{3} m$ como para la $P 4 / m m m$, determinándose así la diferencia de las energías totales $\left(\Delta E=E_{0}(P m \overline{3} m)-E_{0}(P 4 / m m m)\right)$ entre ambas estructuras en sus respectivos volúmenes de equilibrio. Como se puede observar en la Figura 3.22, los átomos X prefieren sustituir a un FeI (sitio $1 a$ de Wyckoff), formándose así compuestos ternarios isoestructurales al $\gamma^{\prime}-\mathrm{Fe}_{4} \mathrm{~N}(\mathrm{Pm} \overline{3} m)$. Para el caso de $X=\mathrm{Ru}$, a pesar que se encontró una pequeña diferencia de energía entre ambas estructura, $\Delta E=-2.52 \mathrm{mRy}$, la sustitución preferencial del átomo Ru en el sitio FeI es corroborado por otros cálculos teóricos [137,142-144]. Mientras que para los otros casos de $X$ considerados, el valor de $\Delta E$ es más notorio. 


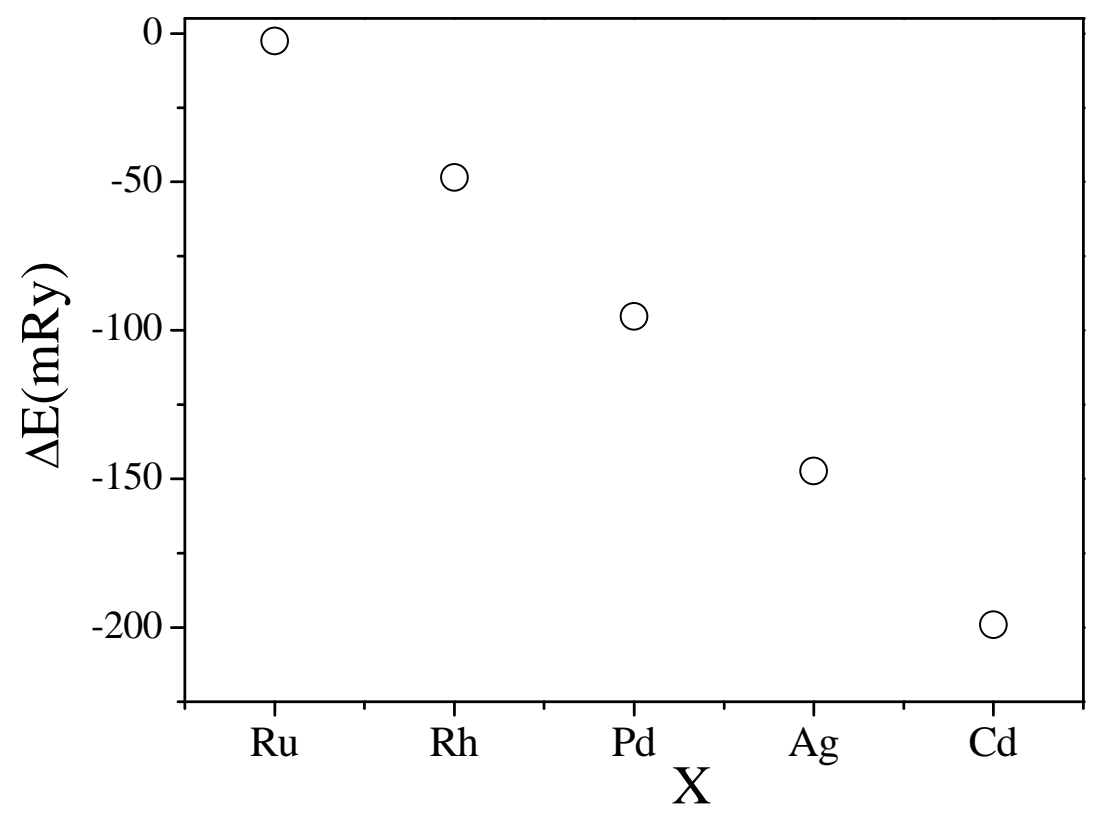

Figura 3.22: Diferencia de energía $(\Delta E)$ entre las dos fases cristalina.

\subsubsection{Propiedades Estructurales}

Sabiendo que los compuestos $\mathrm{XFe}_{3} \mathrm{~N}$ forman la estructura cristalina $P m \overline{3} m$, los datos de volumen versus energía total se ajustaron con la ecuación de estado de Birch-Murnaghan (2.2), ver Figura 3.23, a partir de la cual de obtuvo el volumen de equilibrio y el módulo de bulk para cada compuesto ternario (Tabla 3.9). En la Figura 3.24, se muestra el parámetro de red de cada compuesto ternario en función del número atómico $(Z)$ del átomo $X$, observándose un incremento lineal de $a$, además de una buena correlación con los valores experimentales obtenidos de la bibliografía (Tabla 2.3). 


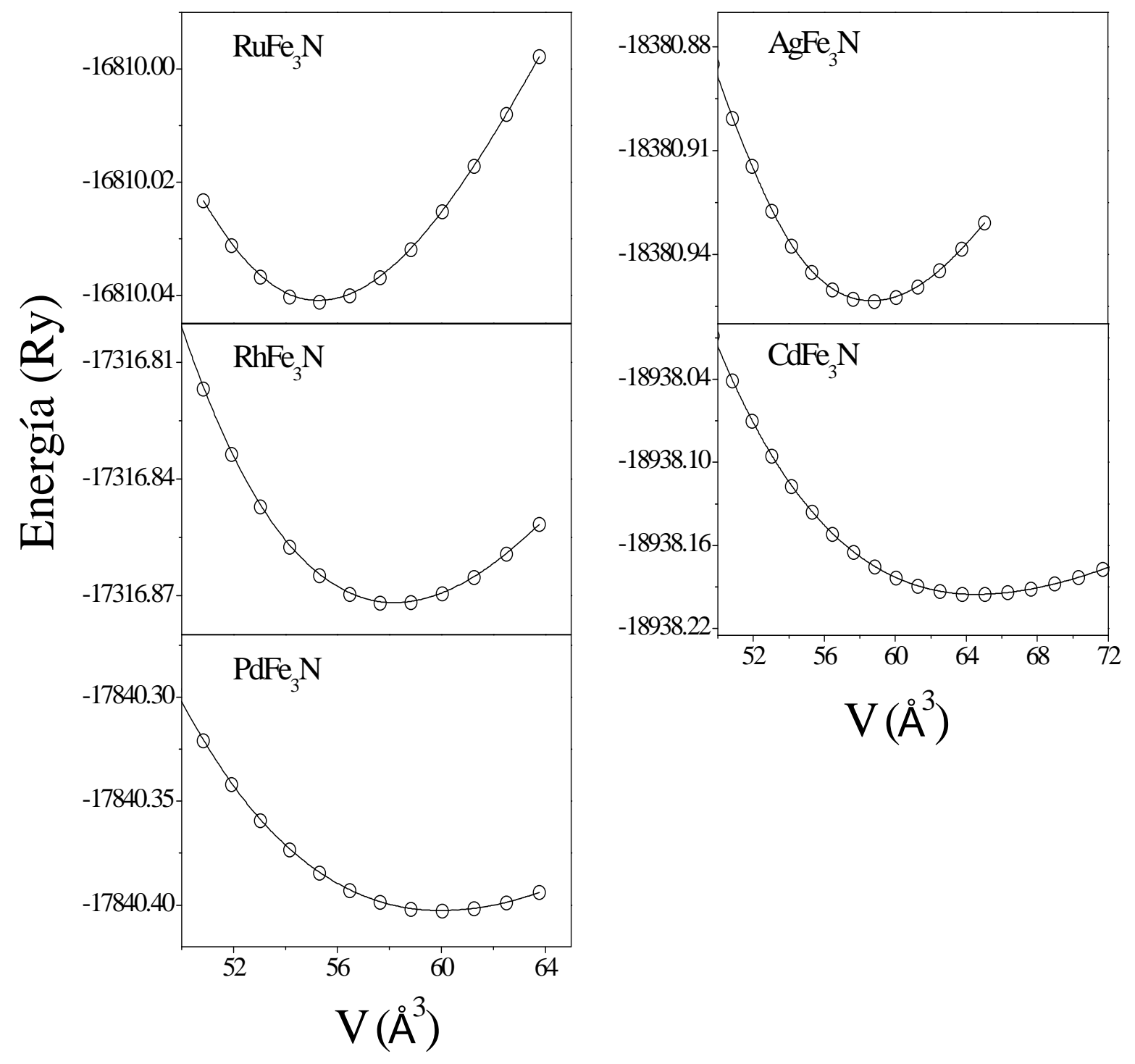

Figura 3.23: Curvas energía total - volumen para los compuestos ternarios $X \mathrm{Fe}_{3} \mathrm{~N}$. 
Tabla 3.9: Volumen $\left(V_{0}\right)$, parámetro de red (a) y módulo de bulk $(B)$ de equilibrio, de los compuestos $\mathrm{XFe}_{3} \mathrm{~N}$ en la fase cristalina $P m \overline{3} m$.

\begin{tabular}{cccc}
\hline$X$ & $V_{0}\left(\AA^{3}\right)$ & $a(\AA)$ & $B(\mathrm{GPa})$ \\
\hline $\mathrm{Ru}$ & 55.211 & 3.8078 & 189.1 \\
$\mathrm{Rh}$ & 56.778 & 3.8435 & 190.0 \\
$\mathrm{Pd}$ & 57.486 & 3.8594 & 189.7 \\
$\mathrm{Ag}$ & 58.846 & 3.8896 & 177.0 \\
$\mathrm{Cd}$ & 59.210 & 3.8976 & 156.0 \\
\hline
\end{tabular}

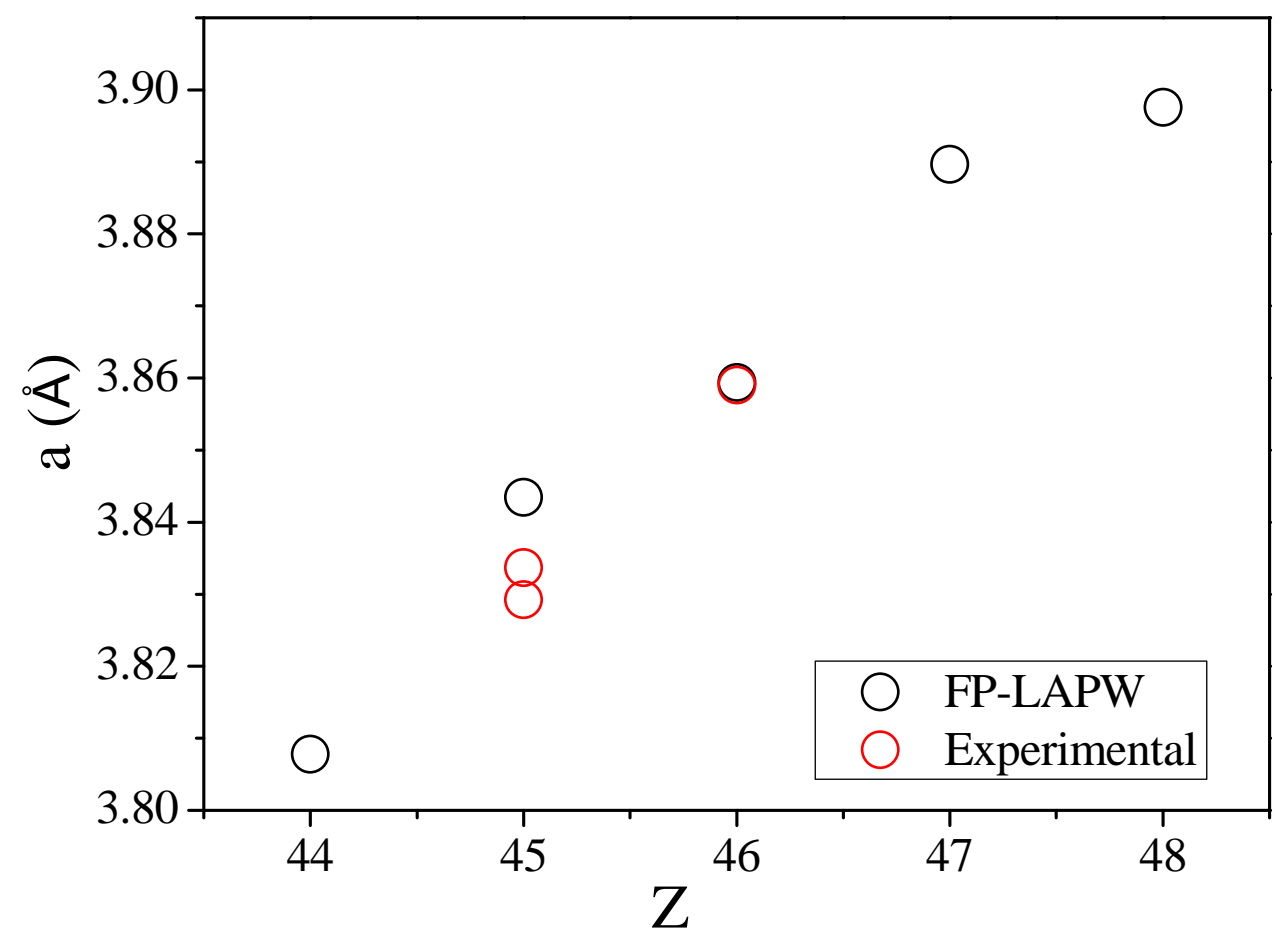

Figura 3.24: Evolución del parámetro de red de los compuestos $\mathrm{XFe}_{3} \mathrm{~N}$, en función del número atómico $(Z)$ del átomo $X$. 
En todos los compuestos $\mathrm{XFe}_{3} \mathrm{~N}$, el parámetro de red de equilibrio es más grande que del $\gamma^{\prime}-\mathrm{Fe}_{4} \mathrm{~N}$ debido a que el radio metálico $\left(r_{m e t}\right)$ de los átomos $X$ son más grandes que del FeI produciendo así una expansión de la celda cristalina, además, en estos compuestos tampoco se encontró una dependencia lineal entre el parámetro de red (a) y el $r_{m e t}$ (Figura 3.25), lo cual demuestra la no existencia de una relación directa entre dichas magnitudes, tal como se determinó en la sección previa.

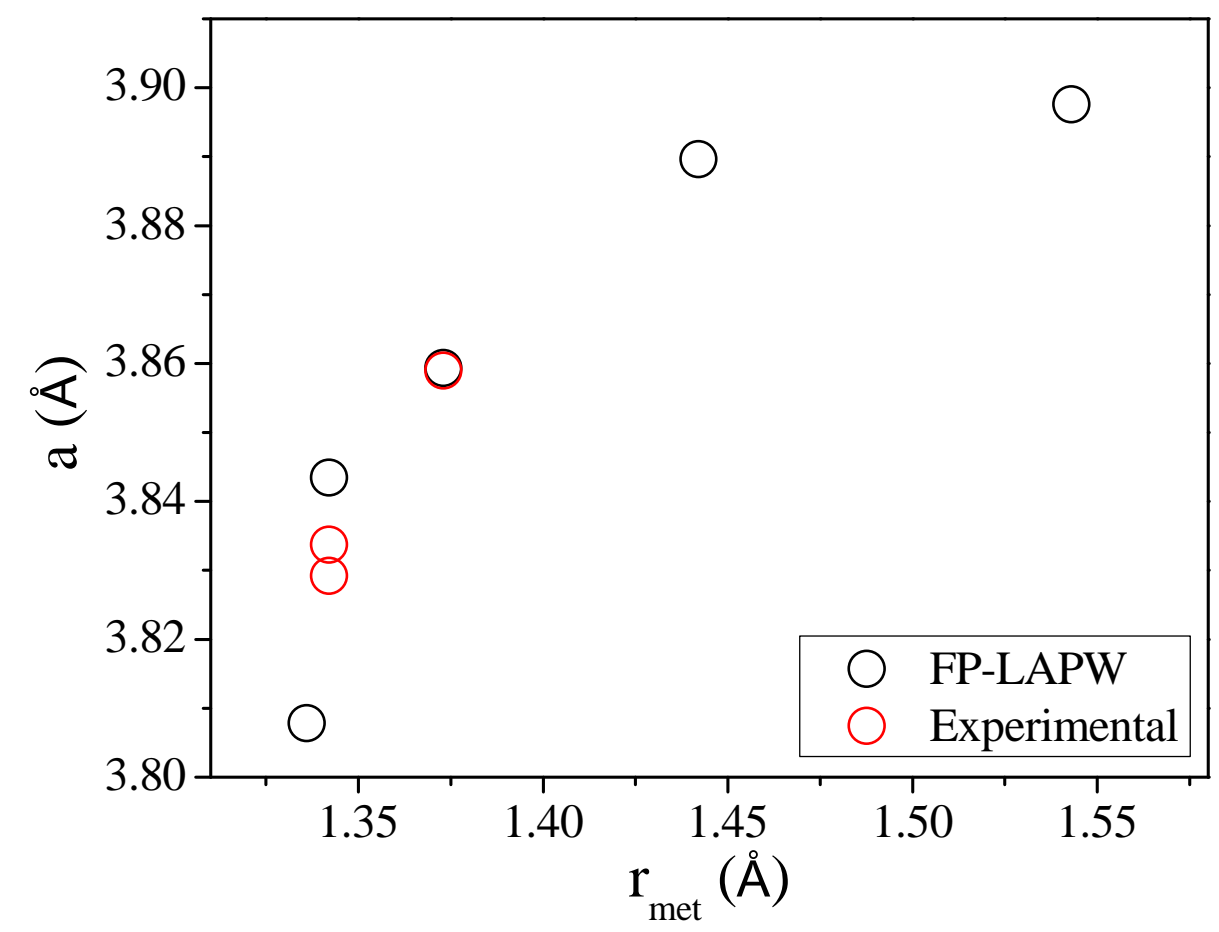

Figura 3.25: Parámetro de red de equilibrio $(a)$ de los compuestos $X \mathrm{Fe}_{3} \mathrm{~N}$ en función del radio metálico $\left(r_{m e t}\right)$ del átomo $X$. 


\subsubsection{Propiedades Magnéticas e Hiperfinas}

La sustitución de un FeI por un átomo $X$, también modifica las propiedades magnéticas e hiperfinas del $\gamma^{\prime}-\mathrm{Fe}_{4} \mathrm{~N}$. En la Tabla 3.10, se reportan los valores de $M_{f u}$ y de los momentos magnéticos de los átomos de Fe y X. El $M_{f u}$ de todos estos compuestos es menor que el del $\gamma^{\prime}-\mathrm{Fe}_{4} \mathrm{~N}$, con un valor máximo para el $\mathrm{RhFe}_{3} \mathrm{~N}$ cuyo valor de $M_{f u}$ está en buen acuerdo con el valor experimental [117].

Tabla 3.10: Valores calculados del $M_{f u}$ y momento magnético por átomo.

\begin{tabular}{cccc}
\hline$X$ & $M_{f u}\left(\mu_{B} / f u\right)$ & $m_{X}\left(\mu_{B}\right)$ & $m_{\mathrm{Fe}}\left(\mu_{B}\right)$ \\
\hline $\mathrm{Ru}$ & 7.45 & 0.68 & 2.12 \\
$\mathrm{Rh}$ & 8.81 & 0.87 & 2.51 \\
$\mathrm{Pd}$ & 8.23 & 0.43 & 2.51 \\
$\mathrm{Ag}$ & 7.43 & 0.10 & 2.37 \\
$\mathrm{Cd}$ & 6.45 & 0.00 & 2.11 \\
\hline
\end{tabular}

En la Figura 3.26a se muestra la variación de $M_{f u}$ en función del $Z$ del átomo $X$, donde se observa un decrecimiento lineal del $M_{f u}$ conforme aumenta el valor de $Z$, este comportamiento no ha sido corroborado con datos experimentales debido a la ausencia de estos. Solo se puede comparar con diferentes datos teóricos obtenidos de la bibliografía (Tabla 2.6), pudiéndose apreciar la misma tendencia del $M_{f u}$. El hecho de que los compuestos $X \mathrm{Fe}_{3} \mathrm{~N}$ tengan un $M_{f u}$ menor al del $\gamma^{\prime}-\mathrm{Fe}_{4} \mathrm{~N}$, está relacionado con la poca contribución magnética de los átomos $X$, los cuales presentan un comportamiento semejante a los compuestos isoestructurales $X \mathrm{Fe}_{3} \mathrm{~N}(X$ es un metal $3 d$ ), es decir, el momento magnético por átomo $X$ va disminuyendo conforme se va llenando el orbital $4 d-X$ (Figura 3.26b) con un valor máximo para el Rh. 


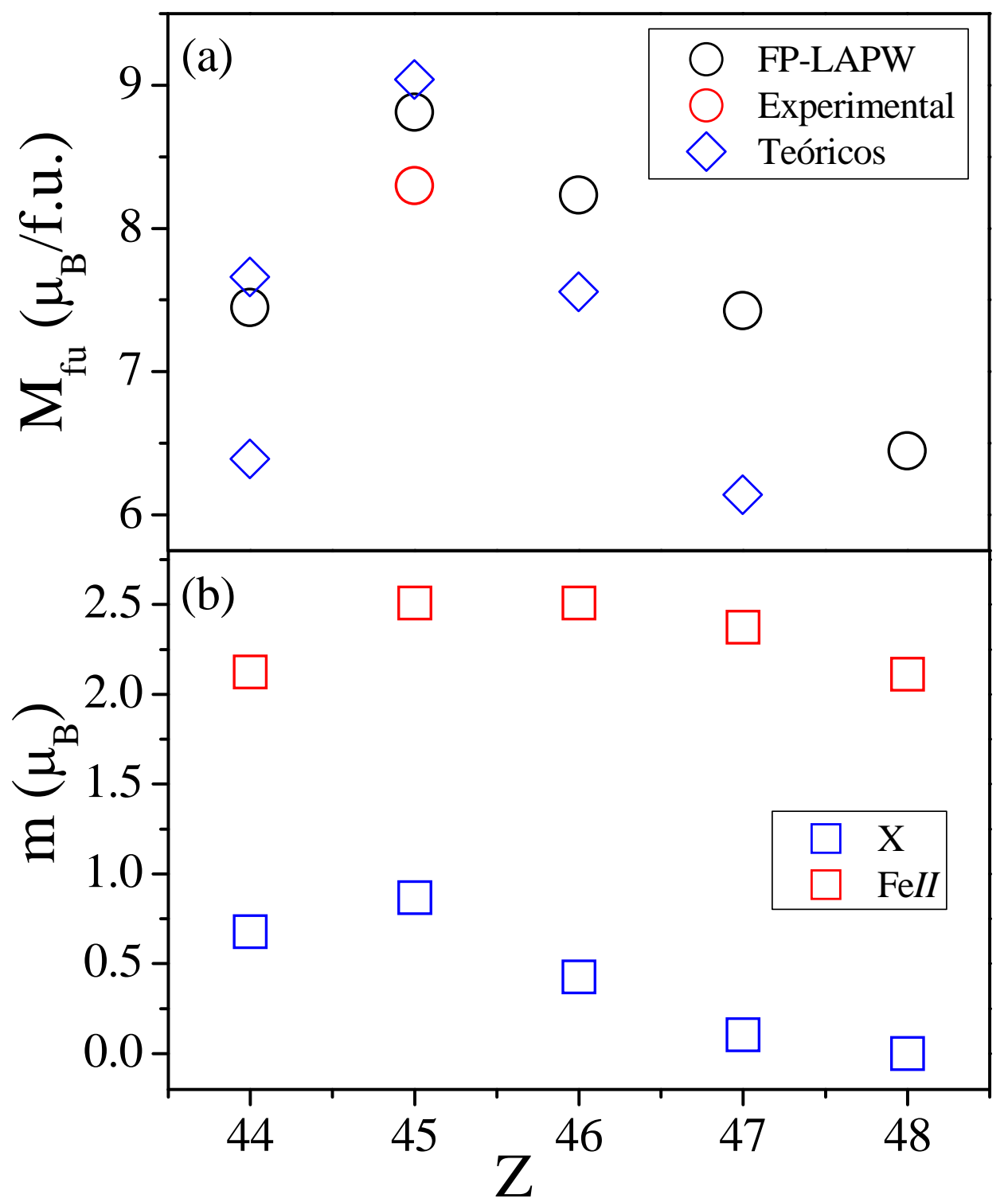

Figura 3.26: Dependencia con $Z$ del a) $M_{f u}$ obtenidos, junto con valores experimentales (Tabla 2.4) y teóricos (Tabla 2.6), b) momento magnético por átomo. 
El incremento del momento magnético entre $X=\mathrm{Rh}$ y Ru está relacionado con el aumento de un electrón en el orbital $4 d-X$, el cual no se encuentran aun totalmente lleno. Mientras que para los demás casos de $X$, la rápida disminución del momento magnético de $X(\mathrm{Pd}, \mathrm{Ag}$ y $\mathrm{Cd})$ se debe a que estos poseen el orbital $4 d$ lleno y el pequeño valor del momento magnético proviene de la disparidad de otros orbitales. Para el caso del compuesto $\mathrm{CdFe}_{3} \mathrm{~N}$, el valor de $M_{f u}$ proviene únicamente de los átomos de Fe, semejante a los compuestos $\mathrm{CuFe}_{3} \mathrm{~N}$ y $\mathrm{ZnFe}_{3} \mathrm{~N}$.

En la Figura 3.27 se muestran las densidades de estados totales y por átomo de los compuestos $X \mathrm{Fe}_{3} \mathrm{~N}$, donde se puede observar en todos los casos la presencia de una hibridización entre los orbitales $3 d$-FeII y los $p$-N en el rango de energía de -8.5 eV y -5.0 eV. Mientras que para el caso de los átomos $X$, se observa una tendencia de los orbitales $4 d-X$ a ocupar estados electrónicos en energías cada vez menores que el $E_{f}$. Para $X=\mathrm{Ru}$, Rh y Pd, se observa una hibridización de los orbitales $4 d-X$ con los $3 d$-FeII en el rango de energía de -5.0 eV y el $E_{f}$, con una mayor población de estados electrónicos cercano al nivel de Fermi para el caso de Ru, mientras que para el Rh y Pd, se observa mayor población de estados electrónicos cercanos a los -5.0 eV. Para el caso de $X=\mathrm{Ag}$, de observa que los estados electrónicos del orbital $4 d$-Ag están concentrados en el rango de $-4.0 \mathrm{eV}$ y $-6.5 \mathrm{eV}$ mostrando una hibridización con los orbitales $2 p-\mathrm{N}$ y $3 d$-FeII, mientras que para el caso de $X=\mathrm{Cd}$, los estados electrónicos $4 d$-Cd se encuentran localizados en un pequeño rango de energías menores de los $-8.5 \mathrm{eV}$, no presentando hibridizaciones con algún orbital del Fe o $\mathrm{N}$. Este desplazamiento de los estados electrónicos $4 d-X$ a menores energías, se ve reflejado en la interacción del átomo $X$ con los de Fe (FeII), produciendo así un decrecimiento del momento magnético de los mismos. 


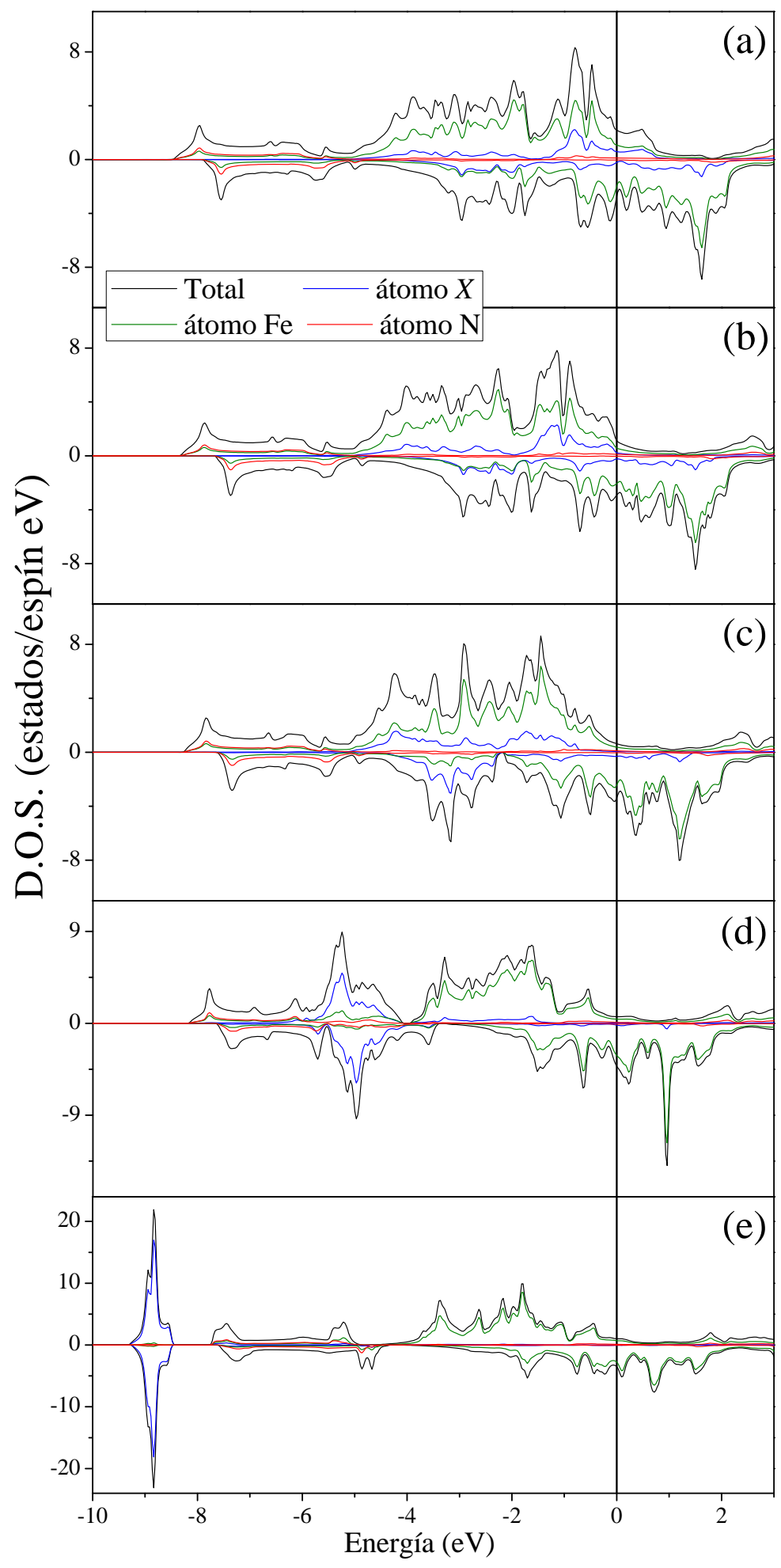

Figura 3.27: Densidad de estados total y por átomo del compuesto ternario a) $\mathrm{RuFe}_{3} \mathrm{~N}$, b) $\left.\left.\mathrm{RhFe}_{3} \mathrm{~N}, \mathrm{c}\right) \mathrm{PdFe}_{3} \mathrm{~N}, \mathrm{~d}\right) \mathrm{AgFe}_{3} \mathrm{~N}$ y e)CdFe ${ }_{3} \mathrm{~N}$. 
La sustitución del FeI por un átomo $X$ en el compuesto $\gamma^{\prime}-\mathrm{Fe}_{4} \mathrm{~N}$ también modifica las propiedades hiperfinas de los átomos FeII debido a la modificación en su distribución de carga electrónica producida por el átomo X. En la Figura 3.28 se muestra la variación del campo hiperfino $\left(B_{h f}\right)$, el corrimiento isomérico $(\delta)$ y cuadrupolar $(\varepsilon)$ del átomo FeII del compuesto $X \mathrm{Fe}_{3} \mathrm{~N}$, en función del $Z$ del átomo $X$, donde se observa que para todos los casos de $X$, el $B_{h f}$ del FeII es siempre menor que al correspondiente FeII del $\gamma^{\prime}-\mathrm{Fe}_{4} \mathrm{~N}$, con una disminución notable del $B_{h f}$ para el caso del $\mathrm{RuFe}_{3} \mathrm{~N}$. Mientras que en el $\mathrm{RhFe}_{3} \mathrm{~N}$ el $B_{h f}$ alcanza su valor máximo, para luego disminuir casi linealmente (Figura 3.28a). En el caso del $\delta$ y $\varepsilon$ (Figura 3.28b y 3.28c, respectivamente) se puede notar un incremento lineal con $Z$ de dichos parámetros, este comportamiento está relacionado con el aumento en la densidad de carga electrónica cercana al núcleo respecto al $\alpha-\mathrm{Fe}(\mathrm{BCC})$ y al aumento de la asimetría esférica de la densidad de carga cercana al núcleo del Fe, respectivamente. Además se observa una buena correlación de los parámetros hiperfinos con los valores experimentales para el $\mathrm{RuFe}_{3} \mathrm{~N}[13], \mathrm{RhFe}_{3} \mathrm{~N}[115,116]$ y $\mathrm{AgFe}_{3} \mathrm{~N}$ [145]. En la Tabla 3.11, se reportan los valores obtenidos de los parámetros hiperfinos de los átomos de Fe de estos compuestos.

Tabla 3.11: Valores calculados del $B_{h f}, \delta$ y $\varepsilon$ del átomo FeII para los diferentes compuestos ternarios $\mathrm{XFe}_{3} \mathrm{~N}$.

\begin{tabular}{cccccc}
\hline$X$ & $\mathrm{Ru}$ & $\mathrm{Rh}$ & $\mathrm{Pd}$ & $\mathrm{Ag}$ & $\mathrm{Cd}$ \\
\hline$B_{h} f(\mathrm{~T})$ & 15.29 & 18.77 & 18.39 & 18.29 & 18.08 \\
$\delta(\mathrm{mm} / \mathrm{s})$ & 0.28 & 0.34 & 0.39 & 0.46 & 0.51 \\
$\varepsilon(\mathrm{mm} / \mathrm{s})$ & 0.12 & 0.16 & 0.22 & 0.23 & 0.28 \\
\hline
\end{tabular}




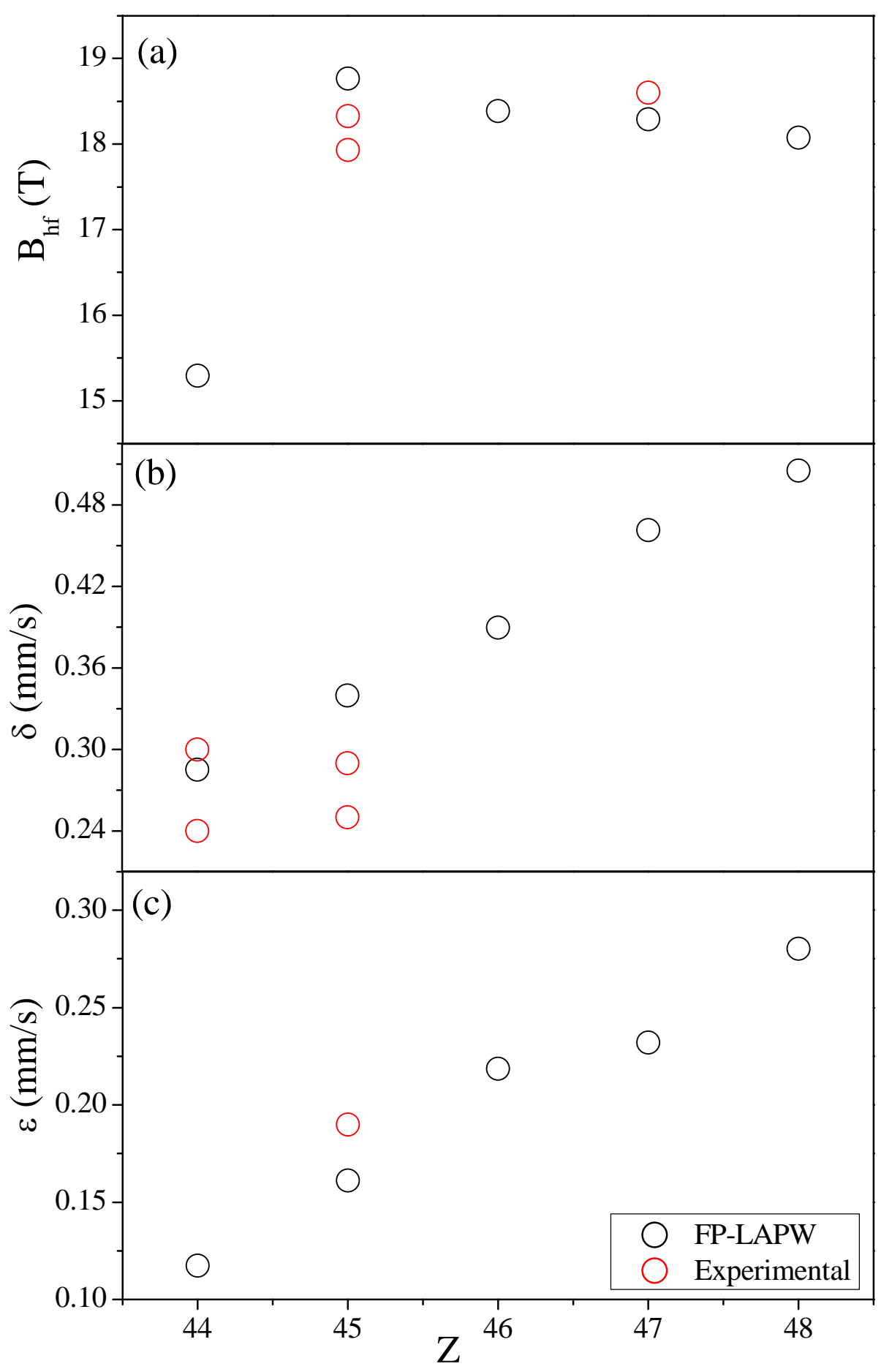

Figura 3.28: Variación del a) $B_{h f}$, b) $\delta$ y c) $\varepsilon$ del átomo de Fe en función del $Z$ del átomo $X$. 


\subsection{Conclusiones}

En el presente capítulo se estudió la variación de las propiedades estructurales, magnéticas e hiperfinas del $\gamma^{\prime}-\mathrm{Fe}_{4} \mathrm{~N}$ al sustituir un átomo de Fe por un átomo $X$ que puede ser un metal de la serie $3 d$ o $4 d$. Debido a que el $\gamma^{\prime}-\mathrm{Fe}_{4} \mathrm{~N}$ posee una estructura cristalina $P m \overline{3} m$ es posible distinguir 2 tipos de Fe (FeI y FeII), inicialmente se analizó un estudio energético sobre el sitio preferencial de sustitución del átomo $X$, llegando a la conclusión que los átomo $3 d$ con un valor de $Z$ mayor al Fe prefieren sustituir a un átomo FeI formando así compuestos ternarios isoestructurales al $\gamma^{\prime}-\mathrm{Fe}_{4} \mathrm{~N}$. A excepción del $\mathrm{ScFe}_{3} \mathrm{~N}$ que forma una estructura cristalina $P m \overline{3} m$, los metales $3 d$ con menor $\mathrm{Z}$ prefieren sustituir a un átomo FeII, formando la estructura cristalina $P 4 / \mathrm{mmm}$ con una celda cristalina tetragonal, donde la razón $c / a$ está relacionada con el $r_{m e t}$ del átomo $X$. Esta sustitución preferencial está relacionada con la afinidad química entre el átomo de $\mathrm{N}$ y el átomo $X$, encontrándose así una buena concordancia con los valores experimentales para los compuestos ternarios $\mathrm{CuFe}_{3} \mathrm{~N}, \mathrm{NiFe}_{3} \mathrm{~N}$ y $\mathrm{ZnFe}{ }_{3} \mathrm{~N}$, mientras que para los compuestos ternarios con estructura $P 4 / \mathrm{mmm}$ se propuso una nueva perspectiva teórica, debido a que trabajos teóricos previos tan solo consideraron que un metal $3 d$ sustituye a un átomo FeI. Para el caso de los metales $4 d$ estudiados, se observó que estos también prefieren sustituir al FeI, incrementando el parámetro de red respecto al del $\gamma^{\prime}-\mathrm{Fe}_{4} \mathrm{~N}$. Esto es una consecuencia a la diferencia en los radios metálicos. Además, no se encontró una relación directa entre el parámetro de red y el radio metálico del átomo $X$.

Respecto a las propiedades magnéticas de estos compuestos ternarios, se encontró una notoria dependencia con la ocupación del orbital $d-X$. Por un lado, los compuestos con estructura $P m \overline{3} m$ presentan un acoplamiento magnético paralelo entre los átomos de Fe y $X$, caracterizándolos como ferromagnetos, pero con un decrecimiento de $M_{f u}$ conforme el orbital $d$-X se va ocupando. Una excepción se presenta en el compuesto $\mathrm{ScFe}_{3} \mathrm{~N}$ el cual es no magnético, pero presenta un posible cambio de fase magnética bajo presión. Por otro lado, los compuestos con estruc- 
tura cristalina $P 4 / \mathrm{mmm}$ presentan una interacción antiparalela entre los átomos de Fe y $X$ con un valor de $M_{f u}$ distinto de cero característico de materiales ferrimagnéticos.

Para el caso de $X$ es un metal $3 d$, el comportamiento magnético de estos compuestos ternarios ha sido descrito satisfactoriamente usando el método de SlaterPauling-Friedel. Además, para el compuesto ternario $\mathrm{MnFe}_{3} \mathrm{~N}$, se realizó un estudio más detallado con el fin de dilucidar su estado fundamental, estructural y magnético. Para lo cual se obtuvo una nueva propuesta teórica sobre su estructura cristalina y respectivo estado magnético fundamental.

Finalmente, se ha encontrado una gran influencia de los metales $X(3 d$ o $4 d$ ) en las propiedades hiperfinas de los átomos de $\mathrm{Fe}$ de $\operatorname{los}$ compuestos $X \mathrm{Fe}_{3} \mathrm{~N}$, tanto para el caso que se encuentren como primeros o segundos vecinos. Dichas propiedades están satisfactoriamente en correlación con los pocos valores experimentales obtenidos de la bibliografía. 


\section{Capítulo 4}

\section{Estudio de los Compuestos}

\section{Ternarios $\boldsymbol{M}_{x} \mathbf{F e}_{4-x} \mathbf{N}(M=\mathbf{M n}, \mathrm{Co}$,}

Ni)

El presente capítulo estará dedicado al estudio de los sistemas ternarios $M_{x} \mathrm{Fe}_{4-x} \mathrm{~N}$, cuando $M$ es un metal $3 d(\mathrm{Mn}, \mathrm{Co}, \mathrm{Ni})$. Como se pudo determinar en el Capítulo 3, el Mn prefiere sustituir a un átomo FeII en la estructura $\gamma^{\prime}-\mathrm{Fe}_{4} \mathrm{~N}$ formándose el compuesto $\mathrm{MnFe}_{3} \mathrm{~N}$, conservándose su forma cúbica, mientras que el Co y Ni prefieren sustituir a un $\mathrm{FeI}$ para formar los compuestos $\mathrm{CoFe}_{3} \mathrm{~N}$ y $\mathrm{NiFe}_{3} \mathrm{~N}$, respectivamente. Motivo por el cual, el desarrollo del presente capítulo se dividirá en dos partes. La primera estará dedicado a determinar el estado fundamental tanto estructural como magnética de los nitruros $\mathrm{Mn}_{x} \mathrm{Fe}_{4-x} \mathrm{~N}$, como también la dependencia con la concentración $(x)$ de Mn del parámetro de red $(a)$ y de las propiedades magnéticas e hiperfinas de dichos compuestos. La segunda parte estará orientada al estudio de la variación de dichas propiedades para los casos en que $M$ sea Co o $\mathrm{Ni}$. 


\subsection{Compuestos Ternarios $\mathrm{Mn}_{x} \mathrm{Fe}_{4-x} \mathbf{N}$}

Como se determinó en el capítulo anterior, el Mn prefiere sustituir a un átomo FeII del $\gamma^{\prime}-\mathrm{Fe}_{4} \mathrm{~N}$ para así formar el $\mathrm{MnFe}_{3} \mathrm{~N}$, donde el momento magnético del $\mathrm{Mn}$ interactúa de forma antiparalela con los átomos de $\mathrm{Fe}$, conllevando a una reducción del momento magnético total a casi la mitad del $\gamma^{\prime}-\mathrm{Fe}_{4} \mathrm{~N}$. En la presente sección se analizará el estado fundamental de los compuestos $\mathrm{Mn}_{x} \mathrm{Fe}_{4-x} \mathrm{~N}$ como también la evolución con la concentración $(x)$ de sus propiedades magnéticas e hiperfinas.

\subsubsection{Determinación del Estado Fundamental}

Con el propósito de determinar el sitio preferencial de sustitución del Mn en los compuestos $\mathrm{Mn}_{x} \mathrm{Fe}_{4-x} \mathrm{~N}$ para los diferentes valores de $x$, se realizaron optimizaciones del parámetro de red $(a)$ a través de la curva energía total - volumen, las cuales fueron ajustadas con la ecuación de estado de Birch-Murnaghan (2.2). Este proceso se realizó para el caso que el átomo de Mn sustituye a átomos FeI o FeII de la supercelda 2 de $\gamma^{\prime}-\mathrm{Fe}_{4} \mathrm{~N}$, los cuales forman las estructuras $P m \overline{3} m$ o $P 4 / m m m$, respectivamente. Para ambos casos los sitios de Fe que serán reemplazados por átomos Mn forman una subred cúbica como se puede ver la Figura 2.2. Además, con el fin de determinar simultáneamente el estado fundamental magnético de estos compuestos ternarios, para cada caso de sustitución se consideró el caso no magnético (NM), ferrimagnético (FE) y ferromagnético (FM). 


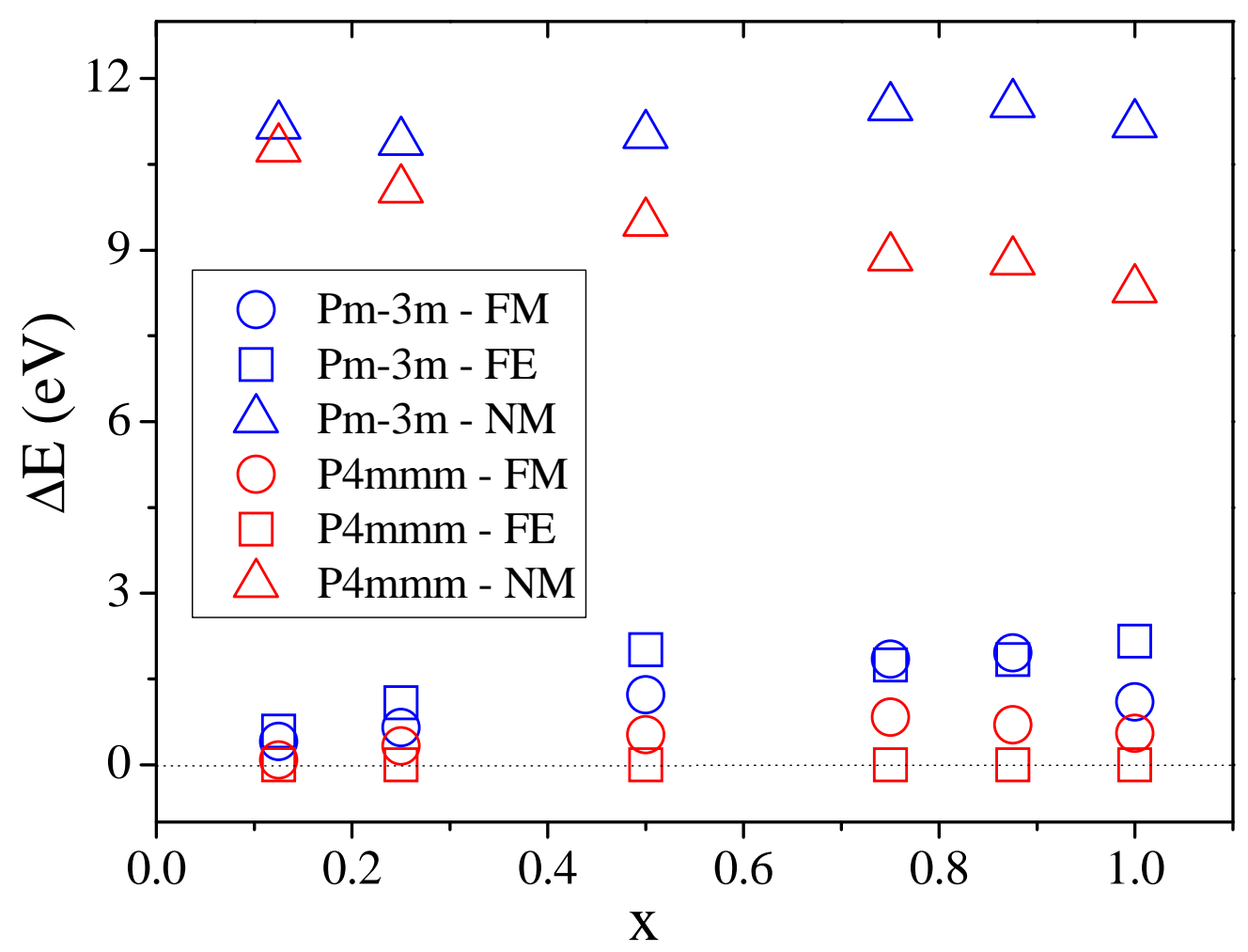

Figura 4.1: Variación de la energía total, respecto al estado $P 4 / \mathrm{mmm}$-FE, de los compuestos $\mathrm{Mn}_{x} \mathrm{Fe}_{4-x} \mathrm{~N}$ para las estructuras $P m \overline{3} m$ y $P 4 / m m m$ con diferentes configuraciones magnéticas.

En la figura 4.1, se muestra la variación de la energía total, perteneciente al volumen de equilibrio, respecto a la configuración $P 4 / \mathrm{mmm}$-FE en función de $x$ la cual es la configuración de mínima energía, seguida de la configuración $P 4 / \mathrm{mmm}$ FM. Para el caso de las $P m \overline{3} m$-FE y $P m \overline{3} m$-FM el $\Delta E$ es más notorio, mientras que el estado NM para ambas estructuras, $P m \overline{3} m$ y $P 4 / m m m$, están a mayores energías. Además, se puede observar que para muy bajas concentraciones de Mn, los estados FE y FM de las estructuras $P m \overline{3} m$ y $P 4 / m m m$ tienden a ser indistinguibles, esto justifica medidas experimentales realizadas en $x=0.10$ [12] donde no fue posible distinguir el sitio preferencial de sustitución del $\mathrm{Mn}$ en el $\gamma^{\prime}-\mathrm{Fe}_{4} \mathrm{~N}$ ni la fase magnética del mismo, concluyendo que el Mn puede sustituir de forma no preferencial cualquiera de los dos sitios cristalográficos (FeI o FeII), descartando también la posibilidad de una sustitución aleatoria [12]. Además, por semejanza 
de los radios atómicos del Fe y el Mn (1.17 $\AA$ para ambos), proponen que el Mn debería de sustituir el FeI sin variar el volumen ni la magnetización del compuesto MnFe3N [12]. Del Capítulo 3, usando cálculos ab-initio, se concluyó que en el compuesto $\mathrm{MnFe}_{3} \mathrm{~N}$ el átomo de $\mathrm{Mn}$ sustituye a un FeII con un parámetro de red semejante al $\gamma^{\prime}-\mathrm{Fe}_{4} \mathrm{~N}$ pero con una magnetización de casi la mitad. Conforme aumenta el valor de $x$, se pueden distinguir ambas estructuras cristalinas, $P m \overline{3} m$ y $P 4 / m m m$, mientras que $\Delta E$ es menor entre el estado FE y FM para la estructura $P 4 / \mathrm{mmm}$. De la Figura 4.1, se puede inferir que para todos los valores de $x$ la configuración de mínima energía es la $P 4 / m m m-F E$, es decir, los átomos de Mn prefieren sustituir a un FeII, interactuando antiparalelamente con los átomos de Fe.

Tabla 4.1: Configuración y parámetro de red de equilibrio $(a)$ de los compuestos $\mathrm{Mn}_{x} \mathrm{Fe}_{4-x} \mathrm{~N}$ correspondiente a la configuración de mínima energía $P 4 / \mathrm{mmm}$-FE.

\begin{tabular}{ccccccc}
\hline$x$ & 0.125 & 0.250 & 0.500 & 0.750 & 0.875 & 1.000 \\
\hline Config. & $\mathrm{C}_{1}^{1}$ & $\mathrm{C}_{18}^{2}$ & $\mathrm{C}_{1368}^{4}$ & $\mathrm{C}_{123678}^{6}$ & $\mathrm{C}_{1234567}^{7}$ & $\mathrm{C}_{12345678}^{8}$ \\
$a(\AA)$ & 3.7961 & 3.7850 & 3.7740 & 3.7752 & 3.7866 & 3.7903 \\
\hline
\end{tabular}

En la Tabla 4.1, se reportan las configuraciones y sus respectivos parámetros de red de equilibrio $(a)$ perteneciente a los diferentes valores de $x$ de los compuestos $\mathrm{Mn}_{x} \mathrm{Fe}_{4-x} \mathrm{~N}$ con estructura P4/mmm-FE. En la Figura 4.2 se muestran dichos valores en función de $x$ junto con las otras configuraciones estructurales y magnéticas de este compuesto, observándose una pequeña disminución de $a$ hasta $x=0.50$, incrementándose luego hasta alcanzar un valor semejante al del $\gamma^{\prime}-\mathrm{Fe}_{4} \mathrm{~N}$. Para bajas concentraciones $(x=0.125)$ los parámetros de red de todas las configuraciones son semejantes, dispersándose luego conforme aumenta $x$. Otra vez, esto concuerda con el único valor experimental a baja concentración [12].

La sustitución de átomos de $\mathrm{Fe}(\mathrm{Fe} I I)$ por $\mathrm{Mn}$ en el $\gamma^{\prime}-\mathrm{Fe}_{4} \mathrm{~N}$, modifican el entorno de los otros átomos de Fe tales como sus primeros $(n n)$ y segundos $(n n n)$ vecinos (Tabla 4.2), esto se ve reflejado tanto en las propiedades estructurales como en las propiedades magnéticas e hiperfinas que se discutirán a continuación. 


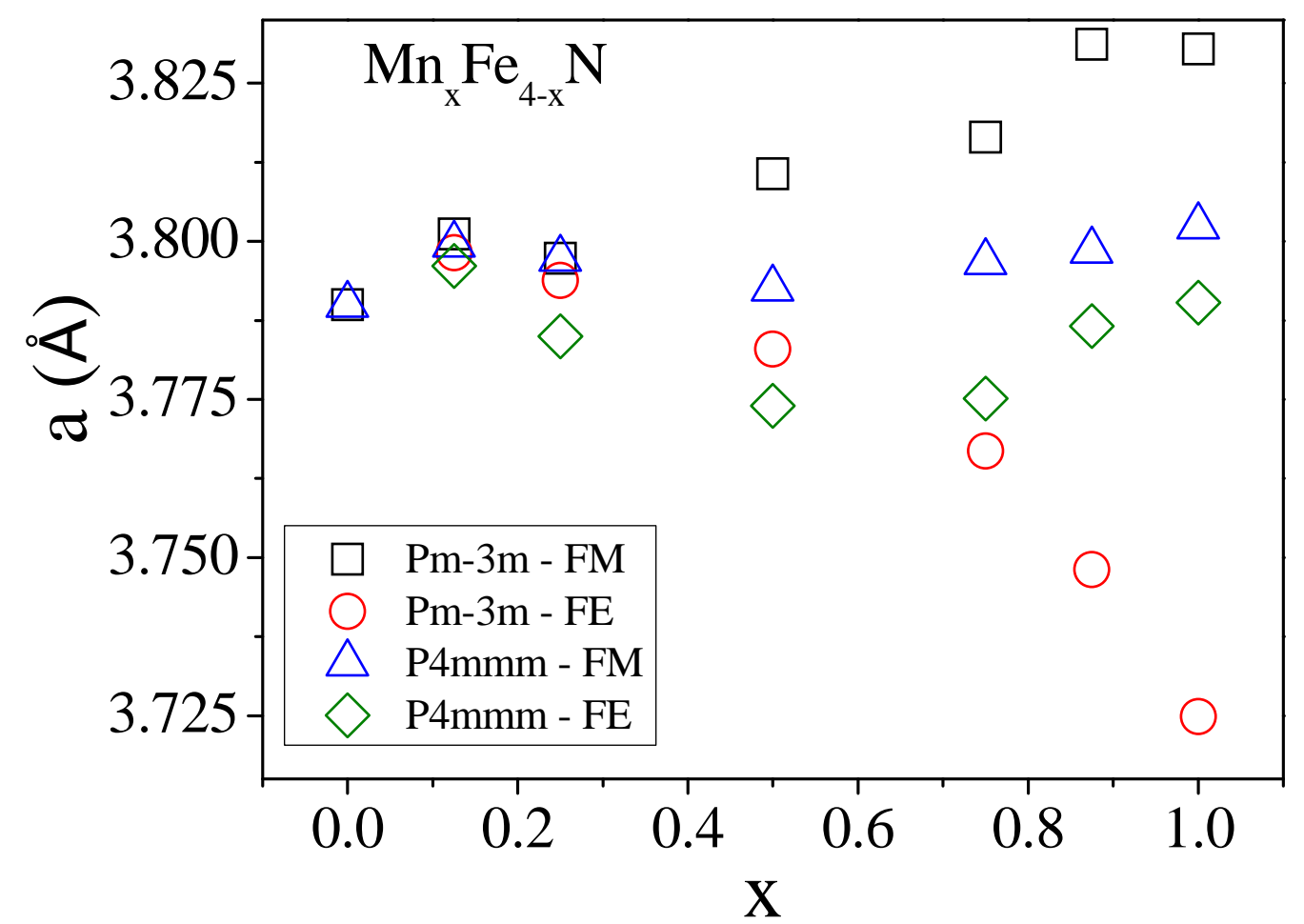

Figura 4.2: Variación del parámetro de red de equilibrio $(a)$ en función de $x$ para las distintas estructuras cristalinas y magnéticas del compuesto $\mathrm{Mn}_{x} \mathrm{Fe}_{4-x} \mathrm{~N}$. 
Tabla 4.2: Diferentes tipos de átomos FeI y FeII respecto a sus primeros $(n n)$ y segundos vecinos (nnn), para las diferentes concentraciones $(x)$ de los compuestos $\mathrm{Mn}_{x} \mathrm{Fe}_{4-x} \mathrm{~N}$. El número en paréntesis representa la cantidad de dicho tipo de átomo.

\begin{tabular}{|c|c|c|c|}
\hline$x$ & & $n n$ & nnn \\
\hline \multirow[t]{2}{*}{0.000} & $\mathrm{Fe} I a(8)$ & $12 \mathrm{Fe} I I a$ & $8 \mathrm{~N}$ \\
\hline & FeIIa(24) & $2 \mathrm{~N}$ & $4 \mathrm{Fe} I a, 8 \mathrm{Fe} I I a$ \\
\hline \multirow[t]{9}{*}{0.125} & FeI $a(4)$ & 4FeIIa, 4FeIIb, 2FeIIc, 1FeIIe, 1Mn & $8 \mathrm{~N}$ \\
\hline & $\mathrm{Fe} I b(4)$ & $4 \mathrm{Fe} I I a, 4 \mathrm{Fe} I I b, 1 \mathrm{Fe} I I d, 2 \mathrm{Fe} I I f, 1 \mathrm{Fe} I I g$ & $8 \mathrm{~N}$ \\
\hline & $\mathrm{Fe} I I a(8)$ & $2 \mathrm{~N}$ & $\begin{array}{l}\text { 1Mn, 2FeIa, 2FeIb, 2FeIIa, 2FeIIb, 1FeIIc, } \\
\text { 1FeIId, 1FeIIf }\end{array}$ \\
\hline & $\mathrm{Fe} I I b(8)$ & $2 \mathrm{~N}$ & $\begin{array}{l}\text { 2FeIa, 2FeIb, 2FeIIa, 2FeIIb, 1FeIId, 1FeIIe, } \\
\text { 1FeIIf, 1FeIIg }\end{array}$ \\
\hline & FeIIc(2) & $2 \mathrm{~N}$ & $4 \mathrm{Fe} I a, 8 \mathrm{Fe} I I a$ \\
\hline & FeIId(2) & $2 \mathrm{~N}$ & $4 \mathrm{Fe} I a, 4 \mathrm{Fe} I I a, 4 \mathrm{Fe} I I b$ \\
\hline & FeIIe(1) & $2 \mathrm{~N}$ & $4 \mathrm{Fe} I b, 8 \mathrm{Fe} I I a$ \\
\hline & FeIIf(2) & $2 \mathrm{~N}$ & $4 \mathrm{Fe} I I a, 8 \mathrm{Fe} I I b$ \\
\hline & FeIIg(1) & $2 \mathrm{~N}$ & $4 \mathrm{Fe} I b, 4 \mathrm{Fe} I I a, 4 \mathrm{Fe} I I b$ \\
\hline \multirow[t]{4}{*}{0.250} & FeI $a(8)$ & $8 \mathrm{Fe} I I a, 1 \mathrm{Mn}, 2 \mathrm{Fe} I I b, 1 \mathrm{Fe} I I c$ & $8 \mathrm{~N}$ \\
\hline & FeIIa(16) & $2 \mathrm{~N}$ & $4 \mathrm{Fe} I a, 4 \mathrm{Fe} I I a, 1 \mathrm{Mn}, 2 \mathrm{Fe} I I b, 1 \mathrm{Fe} I I c$ \\
\hline & $\mathrm{Fe} I I b(4)$ & $2 \mathrm{~N}$ & $4 \mathrm{Fe} I a, 8 \mathrm{Fe} I I a$ \\
\hline & $\mathrm{Fe} I I c(2)$ & $2 \mathrm{~N}$ & $4 \mathrm{Fe} I a, 8 \mathrm{Fe} I I a$ \\
\hline \multirow[t]{4}{*}{0.500} & $\mathrm{Fe} I a(8)$ & $4 \mathrm{Fe} I I a, 4 \mathrm{Fe} I I b, 2 \mathrm{Fe} I I c, 2 \mathrm{Mn}$ & $8 \mathrm{~N}$ \\
\hline & FeIIa(8) & $2 \mathrm{~N}$ & 4FeIa, 4FeIIb, 2FeIIc, 2Mn \\
\hline & $\mathrm{Fe} I I b(8)$ & $2 \mathrm{~N}$ & 4FeIa, 4FeIIa, 2FeIIc, 2Mn \\
\hline & $\mathrm{Fe} I I c(4)$ & $2 \mathrm{~N}$ & $4 \mathrm{Fe} I a, 4 \mathrm{Fe} I I a, 4 \mathrm{Fe} I I b$ \\
\hline \multirow[t]{3}{*}{0.750} & FeIa(8) & 8FeIIa, 3FeIIb, 3Mn & $8 \mathrm{~N}$ \\
\hline & FeIIa(16) & $2 \mathrm{~N}$ & $4 \mathrm{Fe} I a, 4 \mathrm{Fe} I I a, 2 \mathrm{Fe} I I b, 3 \mathrm{Mn}$ \\
\hline & $\mathrm{Fe} I I b(2)$ & $2 \mathrm{~N}$ & $4 \mathrm{Fe} I a, 8 \mathrm{Fe} I I a$ \\
\hline \multirow[t]{5}{*}{0.875} & FeIa(4) & $4 \mathrm{Fe} I I a, 4 \mathrm{Fe} I I b, 1 \mathrm{Fe} I I c, 3 \mathrm{Mn}$ & $8 \mathrm{~N}$ \\
\hline & $\mathrm{Fe} I b(4)$ & $4 \mathrm{Fe} I I a, 4 \mathrm{Fe} I I b, 1 \mathrm{Fe} I I c, 4 \mathrm{Mn}$ & $8 \mathrm{~N}$ \\
\hline & FeIIa(8) & $2 \mathrm{~N}$ & 2FeIa, 2FeIb, 2FeIIa, 2FeIIb, 2FeIIc, 3Mn \\
\hline & $\mathrm{Fe} I I b(8)$ & $2 \mathrm{~N}$ & $2 \mathrm{Fe} I a, 2 \mathrm{Fe} I b, 2 \mathrm{Fe} I I a, 2 \mathrm{Fe} I I b, 4 \mathrm{Mn}$ \\
\hline & $\mathrm{Fe} I I c(1)$ & $2 \mathrm{~N}$ & $4 \mathrm{Fe} I a, 8 \mathrm{Fe} I I a$ \\
\hline \multirow[t]{2}{*}{1.000} & FeIa(8) & $8 \mathrm{Fe} I I a, 4 \mathrm{Mn}$ & $8 \mathrm{~N}$ \\
\hline & FeIIa(16) & $2 \mathrm{~N}$ & $4 \mathrm{Fe} I a, 4 \mathrm{Fe} I I a, 4 \mathrm{Mn}$ \\
\hline
\end{tabular}




\subsubsection{Estructura Electrónica, Propiedades Magnéticas e Hi- perfinas}

La sustitución de átomos de Fe (FeII) por átomos de Mn, ocasiona una redistribución de las densidades de estados (DOS) electrónicos de dichos átomos. En la Fi-

gura 4.3, se muestran las densidades de estados para los compuestos $\mathrm{Mn}_{0.250} \mathrm{Fe}_{3.750} \mathrm{~N}$, $\mathrm{Mn}_{0.500} \mathrm{Fe}_{3.500} \mathrm{~N}$ y $\mathrm{Mn}_{0.750} \mathrm{Fe}_{3.250} \mathrm{~N}$, donde se observa la hibridización entre los orbitales del $\mathrm{N}$ y el FeII en el rango de $-9.0 \mathrm{eV}$ y $-5.0 \mathrm{eV}$, como también una fuerte hibridización entre los orbitales de los FeI y FeII en el rango de energía de -5.0eV y el nivel de Fermi $\left(E_{f}\right)$, estas características son semejantes a las del $\gamma^{\prime}-\mathrm{Fe}_{4} \mathrm{~N}$ [130]. En este mismo rango de energía, se encuentran las densidades de estados de los átomos de Mn, pero con la diferencia que la mayoría de los estados electrónicos están en la densidad de estados down, mientras que las de los átomos de Fe tienen mayormente poblado las densidades de estados up, indicando el tipo de acoplamiento magnético antiparalelo entre dichas especies atómicas. En la Figura 4.4, se muestra la diferencia entre las densidades de carga up y down para $x=0.125,0.500$ y 0.750 , pertenecientes al plano (001) de la respectiva supercelda utilizada, mostrando así la distribución de carga de los átomos de Fe (FeI y FeII) y Mn las cuales están directamente relacionada con el momento magnético atómico, dando indicio del tipo de acoplamiento magnético antiparalelo existente entre estas especies atómicas. 


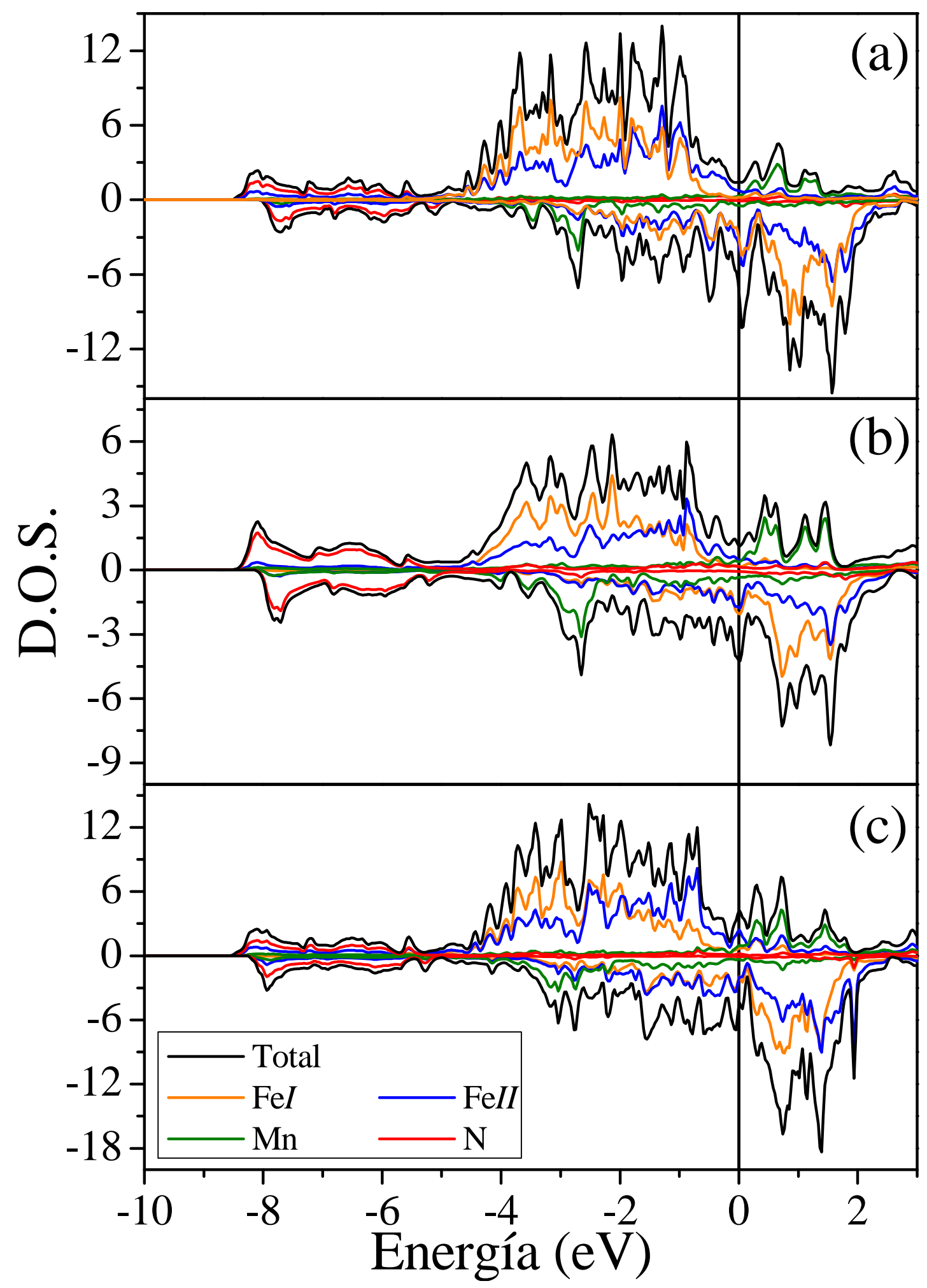

Figura 4.3: Densidad de estados totales y por átomo de los compuestos a) $\mathrm{Mn}_{0.250} \mathrm{Fe}_{3.750} \mathrm{~N}$, b) $\mathrm{Mn}_{0.500} \mathrm{Fe}_{3.500} \mathrm{~N}$ y c) $\mathrm{Mn}_{0.750} \mathrm{Fe}_{3.250} \mathrm{~N}$. 

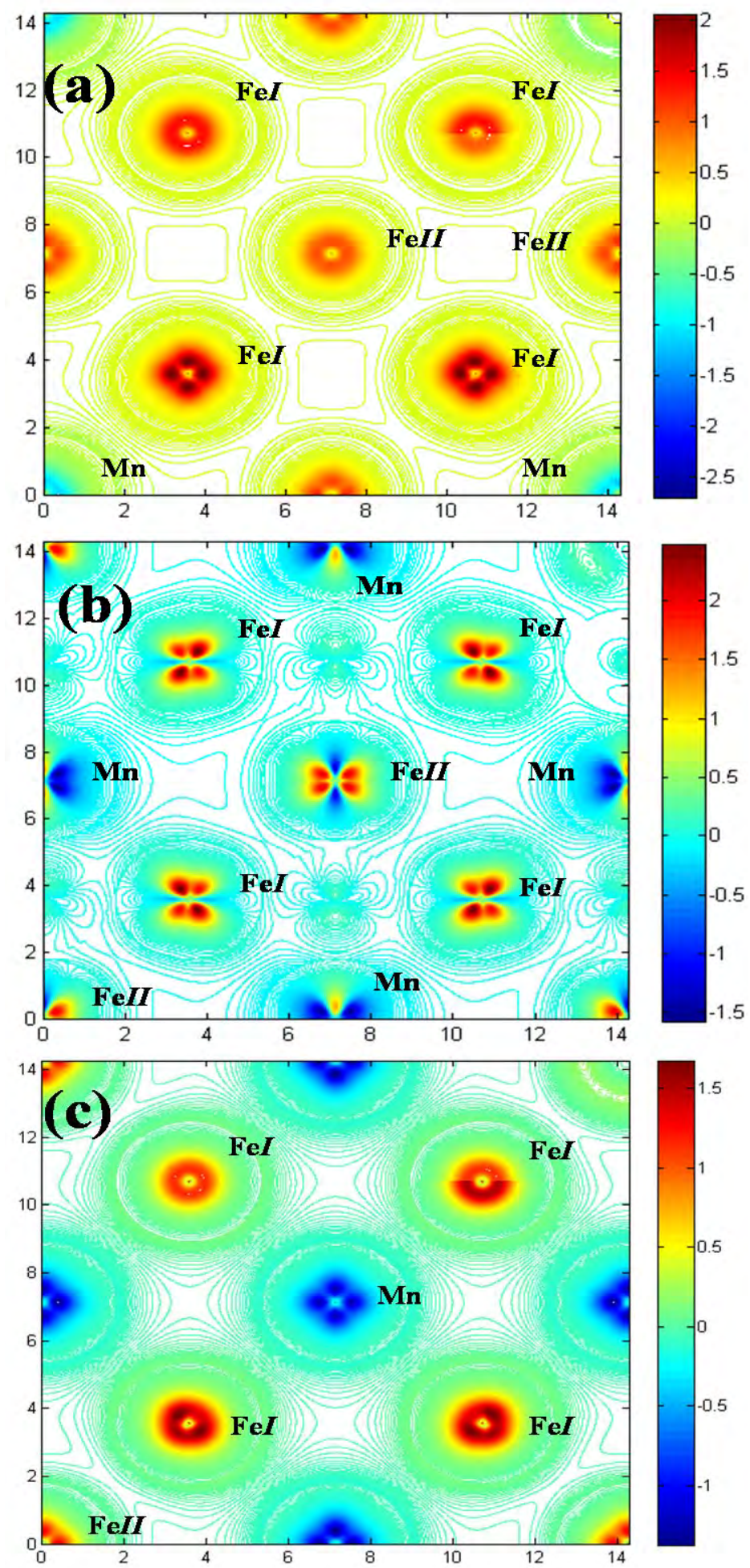

Figura 4.4: Diferencia de las densidades de carga up y down para los compuestos a) $\left.\mathrm{Mn}_{0.125} \mathrm{Fe}_{3.875} \mathrm{~N}, \mathrm{~b}\right) \mathrm{Mn}_{0.500} \mathrm{Fe}_{3.500} \mathrm{~N}$ y c) $\mathrm{Mn}_{0.875} \mathrm{Fe}_{3.125} \mathrm{~N}$. 
El arreglo magnético antiparalelo entre los átomos de Mn y Fe hacen que el momento magnético por fórmula unidad $\left(M_{f u}\right)$ de los compuestos $\mathrm{Mn}_{x} \mathrm{Fe}_{4-x} \mathrm{~N}$ disminuya con $x$. En la Figura 4.5a se muestran las variaciones de $M_{f u}$ con $x$ para la configuración de mínima energía $(P 4 / m m m-F E)$ junto con las otras configuraciones, por comparación. El $M_{f u}$ del estado FM para ambas estructuras cristalinas son muy semejantes y no varían con $x$. Para la configuración $P m \overline{3} m$-FE, se observa una dependencia parabólica decreciente de $M_{f u}$ con $x$, mientras que para la configuración de mínima energía $P 4 / \mathrm{mmm}$-FE se puede observar una disminución lineal de $M_{f u}$ con $x$, con pendiente de $-5.43 \mu_{B}$, conllevando a una reducción del $50 \%$ del $M_{f u}$ respecto del $\gamma^{\prime}-\mathrm{Fe}_{4} \mathrm{~N}$ para el caso del $\mathrm{MnFe}{ }_{3} \mathrm{~N}$.

La disminución lineal de $M_{f u}$ con $x$ de la configuración $P 4 / m m m-F E$, está relacionada con el tipo de interacción antiparalela existente entre los átomos de Fe y Mn, lo cual ocasiona una disminución del momento magnético de los átomos de Fe debido a la redistribución de su estructura electrónica, ocasionando un desplazamiento de las densidades de estados up hacia energías mayores que la $E_{f}$, simultáneamente las densidades de estados down se desplazan en dirección opuesta (Figura 4.3). En la Figura 4.5b se muestra la variación del momento magnético de los átomos FeI y FeII con la concentración de Mn, $(x)$, donde se observa una decrecimiento lineal con pendientes de $-0.31 \mu_{B}$ y $-0.38 \mu_{B}$, respectivamente. Mientras que el átomo de Mn posee un momento magnético de $-2.3 \mu_{B}$ el cual no varía con $x$. Debido a que el $M_{f u}$ es distinto de cero a pesar del tipo de acoplamiento magnético existente entre los átomos de Fe y Mn, estos compuestos pueden ser caracterizados como ferrimagnetos. 


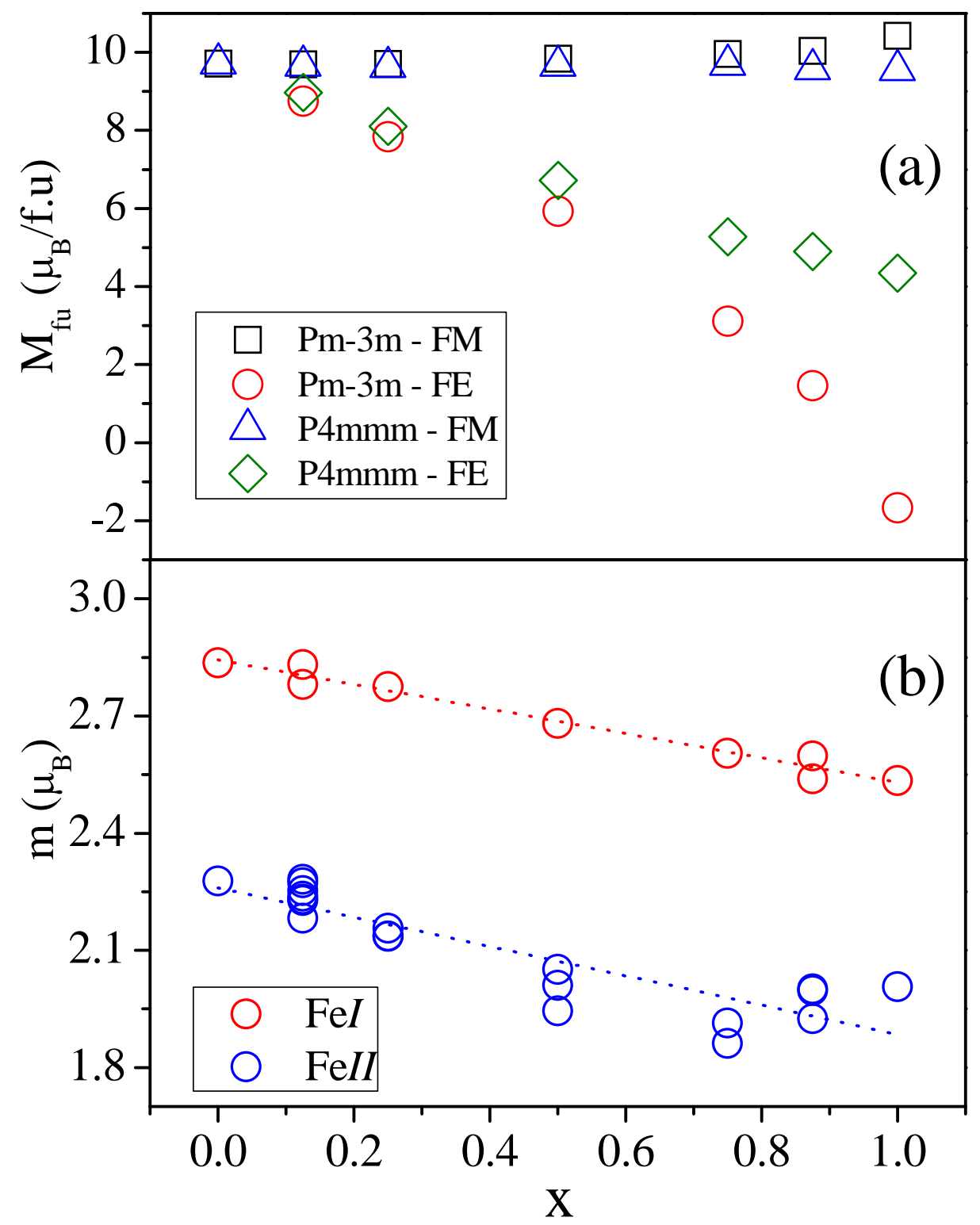

Figura 4.5: Variación con $x$ de la a) $M_{f u}$ de las estructuras $P m \overline{3} m$ y $P 4 / m m m$ para los estados FE y FM, b) momento magnético de los átomos de Fe de la configuración de mínima energía $P 4 / m m m$-FE. 
Respecto a las propiedades hiperfinas de estos compuestos ternarios, se han sido obtenidos considerando lo detallado en el Capítulo 2. En la Figura 4.6 se muestran la variación del campo hiperfino $\left(B_{h f}\right)$, el corrimiento isomérico $(\delta)$ y el desdoblamiento cuadrupolar $(\varepsilon)$ de los átomos de Fe. Se observa que el $B_{h f}$ (Figura 4.6a) de los FeI y FeII disminuyen linealmente con $x$ con pendientes de $-2.3 T$ y $-1.1 T$ respectivamente. Esto indica una mayor influencia de los átomos en los FeI, produciendo una mayor polarización de los estados electrónicos cercanos al núcleo, esto ocurre a pesar de que los FeI y FeII se encuentran a la misma distancia interatómica con el átomo de Mn. Mientras que el $\delta$ de los FeI muestra un pequeño decrecimiento lineal con $x$, lo cual representa la existencia de variaciones en la ocupación de los estados electrónicos cercanos al núcleos, respecto del $\alpha-\mathrm{Fe}(\mathrm{BCC})$. En el caso de los átomos FeII, el $\delta$ no varía con $x$, manteniendo un valor semejante al FeII del $\gamma^{\prime}-\mathrm{Fe}_{4} \mathrm{~N}$ (Figura 4.6b). Concerniente al $\varepsilon$ de los FeI y FeII, los valores absolutos de $\varepsilon$ no varían con $x$ cuyos valores son semejantes al del $\gamma^{\prime}-\mathrm{Fe}_{4} \mathrm{~N}$ (Figura 4.6c). Para $x$ mayores de 0.500 , se observa el cambio de signo del $\varepsilon$ en algunos FeII, esto está relacionado con un cambio de la forma oblicua de la distribución de carga cercana al núcleo de los dichos átomos. 


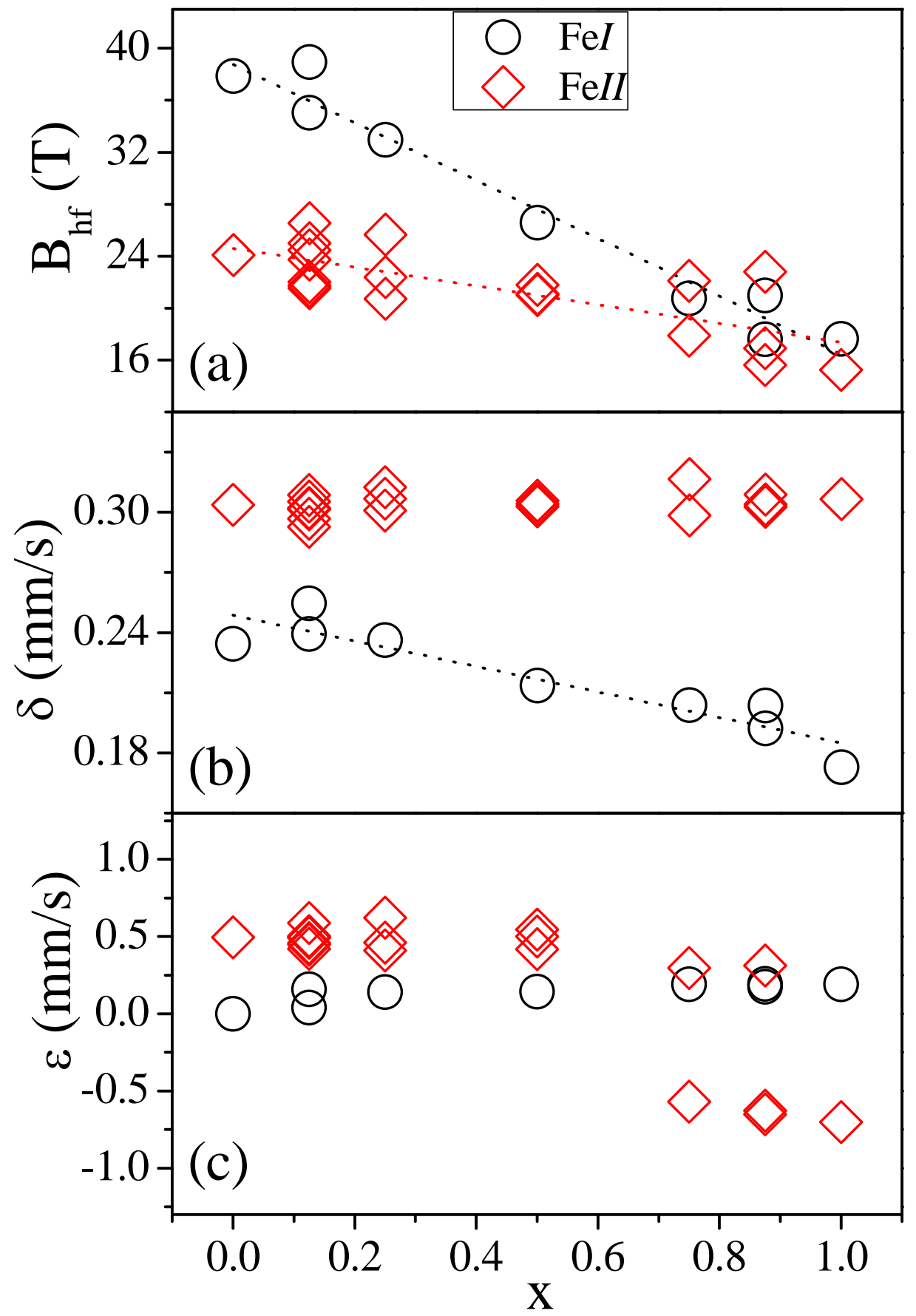

Figura 4.6: Dependencia con $x$ del a) $B_{h f}$, b) $\delta$ y c) $\varepsilon$ de los átomos FeI y FeII de los compuestos $\mathrm{Mn}_{x} \mathrm{Fe}_{4-x} \mathrm{~N}$. 
Complementariamente, para poder estudiar la influencia de las propiedades magnéticas en las hiperfinas, se determinó la constante de acoplamiento hiperfino $(A)$, a partir de la expresión (2.8). En la Figura 4.7 se muestran los valores del momento magnético versus el $B_{h f}$ de los átomos FeI de estos compuestos ternarios, de los cuales se determinó que $A=73.6 T / \mu_{B}$, esto indica una alta dependencia y sensibilidad de los parámetros hiperfinos del FeI a los efectos magnéticos procedentes de los átomos que se encuentran en su entorno.

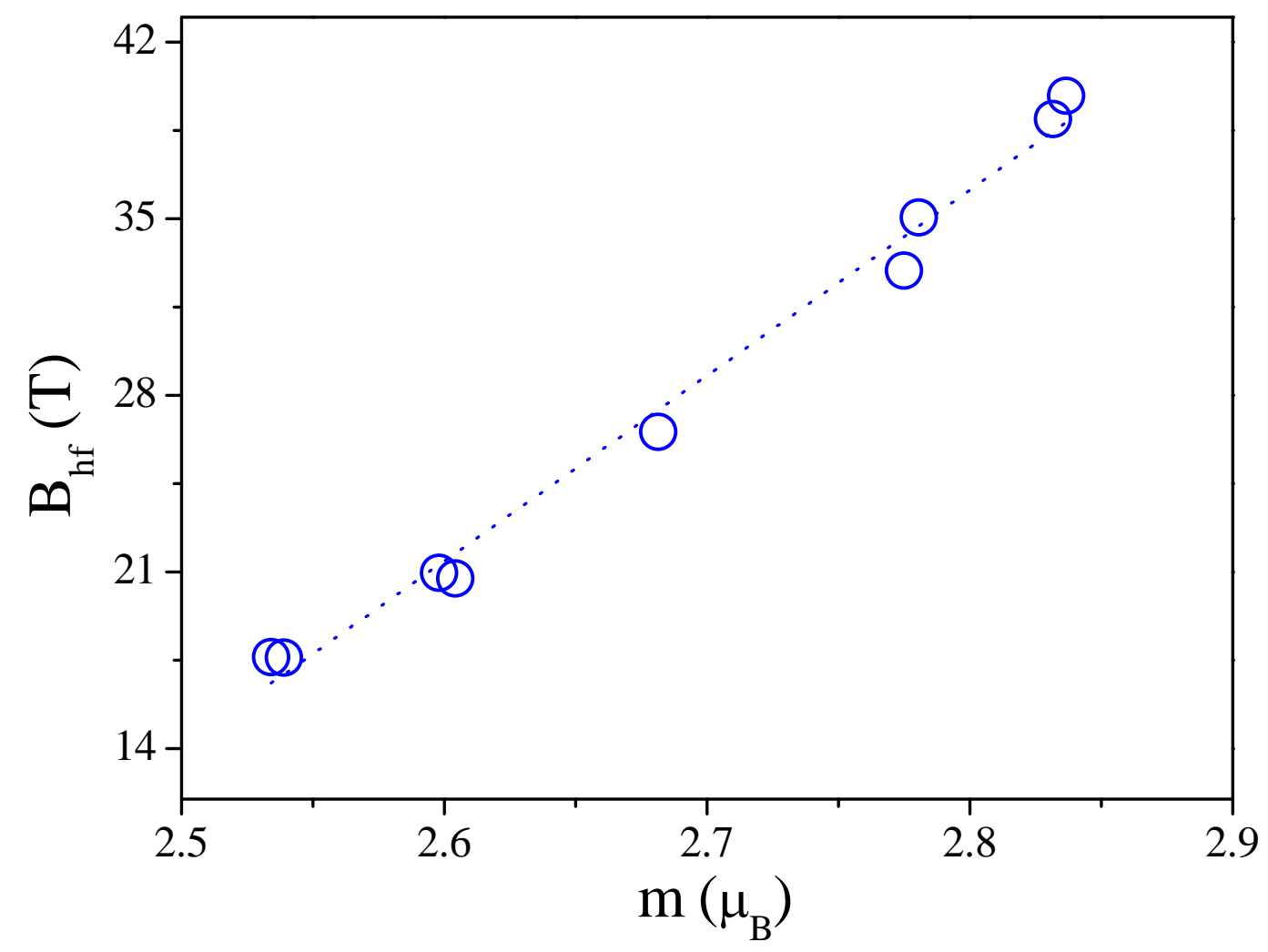

Figura 4.7: Dependencia lineal entre el momento magnético $(m)$ y el campo hiperfino $\left(B_{h f}\right)$ de los átomos FeI de los compuestos ternarios $\mathrm{Mn}_{x} \mathrm{Fe}_{4-x} \mathrm{~N}$.

\subsection{Compuestos Ternarios $\mathrm{Co}_{x} \mathrm{Fe}_{4-x} \mathbf{N}$ y Ni $\mathrm{Fe}_{4-x} \mathbf{N}$}

En el Capítulo 3, se determinó que el Co y el Ni prefieren sustituir de forma preferencial al FeI en el compuesto $\gamma^{\prime}-\mathrm{Fe}_{4} \mathrm{~N}$ para formar los compuestos $\mathrm{CoFe}_{3} \mathrm{~N}$ 
y $\mathrm{NiFe}_{3} \mathrm{~N}$, respectivamente. Para el último caso, se ha podido corroborar con datos experimentales obtenidos de la bibliografía. Con el fin de estudiar la variación del parámetro de red $(a)$ y de las propiedades magnéticas e hiperfinas con la concentración $(x)$ en los compuestos $\mathrm{Co}_{x} \mathrm{Fe}_{4-x} \mathrm{~N}$ y $\mathrm{Ni}_{x} \mathrm{Fe}_{4-x} \mathrm{~N}$, se utilizó una supercelda $2 \times 2 \times 2$ de $\gamma^{\prime}-\mathrm{Fe}_{4} \mathrm{~N}$, en el cual tan solo se ha considerado el caso donde el átomo de Co o Ni sustituye a los FeI.

\subsubsection{Propiedades Estructurales}

En la supercelda $2 \times 2 \times 2$ de $\gamma^{\prime}-\mathrm{Fe}_{4} \mathrm{~N}$, se forma una subred cúbica de átomos $\mathrm{Fe} I$ los cuales han sido sustituidos por átomos $\mathrm{M}(\mathrm{M}=\mathrm{Co}, \mathrm{Ni})$ obteniéndose diferentes valores de $x$. Para cada valor de $x$, se determinó la curva energía total - volumen y en los casos donde existe más de una configuración posible para obtener el mismo valor de $x$, se consideró la configuración de mínima energía. En la Tabla 4.3, para cada valor de $x$, se reporta la configuración de mínima energía y su respectivo parámetro de red de equilibrio $(a)$ y módulo de bulk (B).

En la Figura 4.8 se muestra la variación del parámetro de red $(a)$ en función de $x$ para los compuestos $\mathrm{Co}_{x} \mathrm{Fe}_{4-x} \mathrm{~N}$ (Figura 4.8a) y $\mathrm{Ni}_{x} \mathrm{Fe}_{4-x} \mathrm{~N}$ (Figura 4.8a) observándose una dependencia no lineal entre dichas variables. Esto es, el parámetro de red decrece con $x$ hasta $x=0.750$, para luego incrementar hasta $x=1.000$. 
Tabla 4.3: Configuración de mínima energía, parámetro de red de equilibrio $(a)$ y su respectivo módulo de bulk (B) de los compuestos $\mathrm{Co}_{x} \mathrm{Fe}_{4-x} \mathrm{~N}_{\text {y Ni}} \mathrm{Fe}_{4-x} \mathrm{~N}$.

\begin{tabular}{ccccccccc}
\hline \multicolumn{8}{c}{$\mathrm{Co}_{x} \mathrm{Fe}_{4-x} \mathrm{~N}$} \\
$x$ & 0.125 & 0.250 & 0.375 & 0.500 & 0.625 & 0.750 & 0.875 & 1.00 \\
\hline Config. & $\mathrm{C}_{1}^{1}$ & $\mathrm{C}_{18}^{2}$ & $\mathrm{C}_{258}^{3}$ & $\mathrm{C}_{1346}^{4}$ & $\mathrm{C}_{13467}^{5}$ & $\mathrm{C}_{123678}^{6}$ & $\mathrm{C}_{2345678}^{7}$ & $\mathrm{C}_{12345678}^{8}$ \\
$a(\AA)$ & 3.7909 & 3.7897 & 3.7865 & 3.7836 & 3.7793 & 3.7718 & 3.7735 & 3.7784 \\
$\mathrm{~B}(\mathrm{GPa})$ & 177.0 & 178.0 & 180.2 & 180.1 & 181.1 & 184.1 & 184.5 & 187.0 \\
\hline & & & & $\mathrm{Ni}_{x} \mathrm{Fe}_{4-x} \mathrm{~N}$ & & & \\
$x$ & 0.125 & 0.250 & 0.375 & 0.500 & 0.625 & 0.750 & 0.875 & 1.00 \\
\hline Config. & $\mathrm{C}_{1}^{1}$ & $\mathrm{C}_{23}^{2}$ & $\mathrm{C}_{258}^{3}$ & $\mathrm{C}_{1346}^{4}$ & $\mathrm{C}_{13467}^{5}$ & $\mathrm{C}_{123678}^{6}$ & $\mathrm{C}_{2345678}^{7}$ & $\mathrm{C}_{12345678}^{8}$ \\
$a(\AA)$ & 3.7915 & 3.7909 & 3.7892 & 3.7851 & 3.7807 & 3.7746 & 3.7762 & 3.7812 \\
B (GPa) & 178.7 & 180.8 & 183.5 & 184.8 & 187.5 & 188.7 & 189.4 & 192.1 \\
\hline
\end{tabular}

Este peculiar comportamiento está relacionado con los compuestos denominados not completely normal or inverse (no completamente normales o inversos) [182184], observados también en otras aleaciones de Fe-Co [185-187] y Fe-Ni [188,189]. Para el caso del $\mathrm{Co}_{x} \mathrm{Fe}_{4-x} \mathrm{~N}$, lamentablemente no existe mucha información experimental [148] con que comparar, mientras que para los compuestos $\mathrm{Ni}_{x} \mathrm{Fe}_{4-x} \mathrm{~N}$ se observa una muy buena correlación con los diferentes valores experimentales obtenidos de la bibliografía [103-110]. En ambos compuestos, los valores de $a$ son siempre menores al del $\gamma^{\prime}-\mathrm{Fe}_{4} \mathrm{~N}$, esto se debe a la diferencia entre los radios metálicos $\left(r_{m e t}\right)$ del Fe $(1.260 \AA)$ y del átomo Co $(1.252 \AA)$ o Ni $(1.244 \AA)$ [190]. Concerniente al módulo de bulk (B), se encontró un aumento lineal de B con $x$ (Figura 4.9) con pendientes de 11.1GPa y 16.0GPa para los compuestos $\mathrm{Co}_{x} \mathrm{Fe}_{4-x} \mathrm{~N}$ y $\mathrm{Ni}_{x} \mathrm{Fe}_{4-x} \mathrm{~N}$, respectivamente, indicando un mejoramiento de la resistencia a la comprensión uniforme, respecto del $\gamma^{\prime}-\mathrm{Fe}_{4} \mathrm{~N}$. 


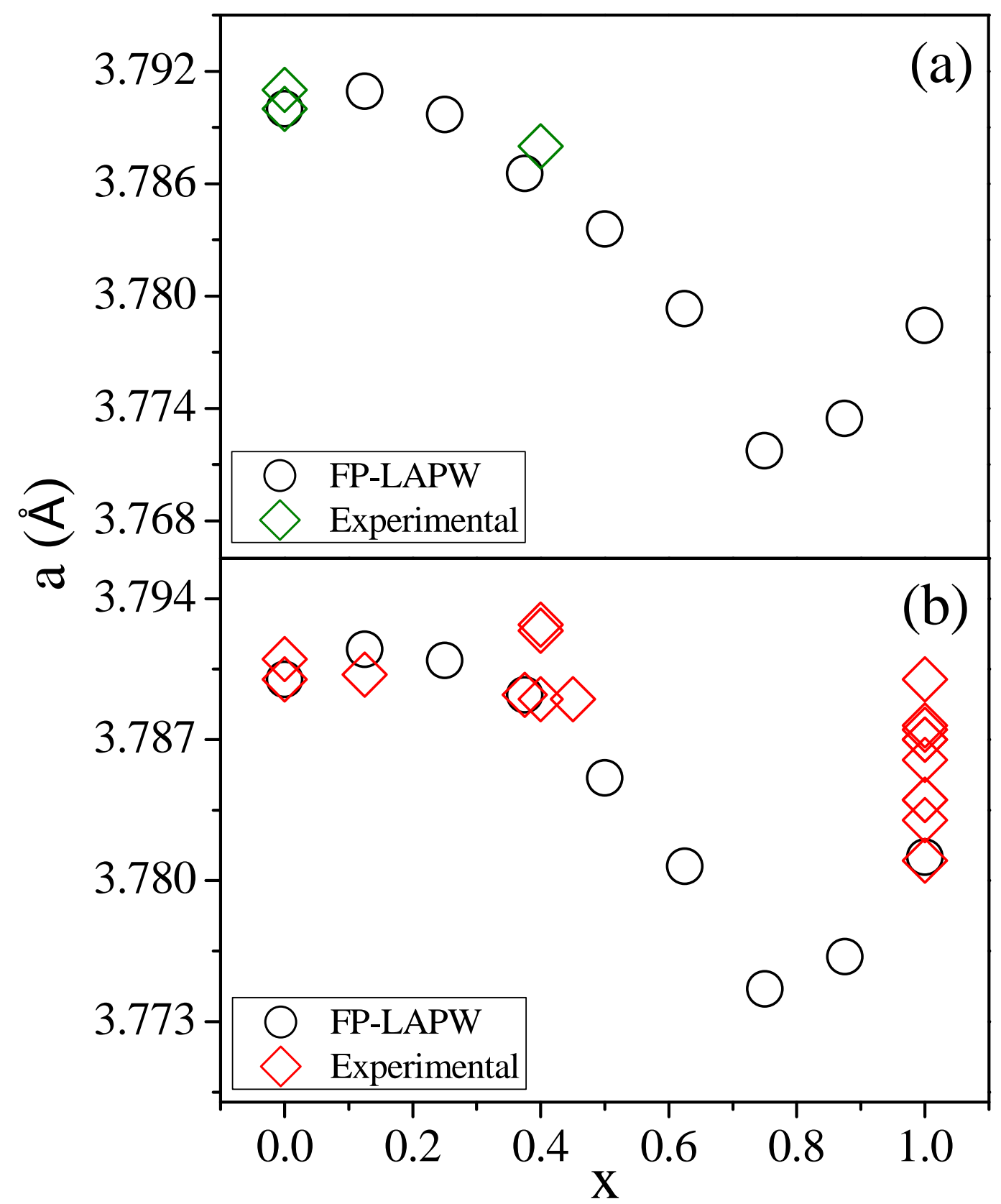

Figura 4.8: Variación del parámetro de red $(a)$ con la concentración $(x)$ para los compuestos a) $\mathrm{Co}_{x} \mathrm{Fe}_{4-x} \mathrm{~N}$, junto con los valores experimentales obtenidos de [148], b) $\mathrm{Ni}_{x} \mathrm{Fe}_{4-x} \mathrm{~N}$, junto con los valores experimentales reportados [103-110]. 


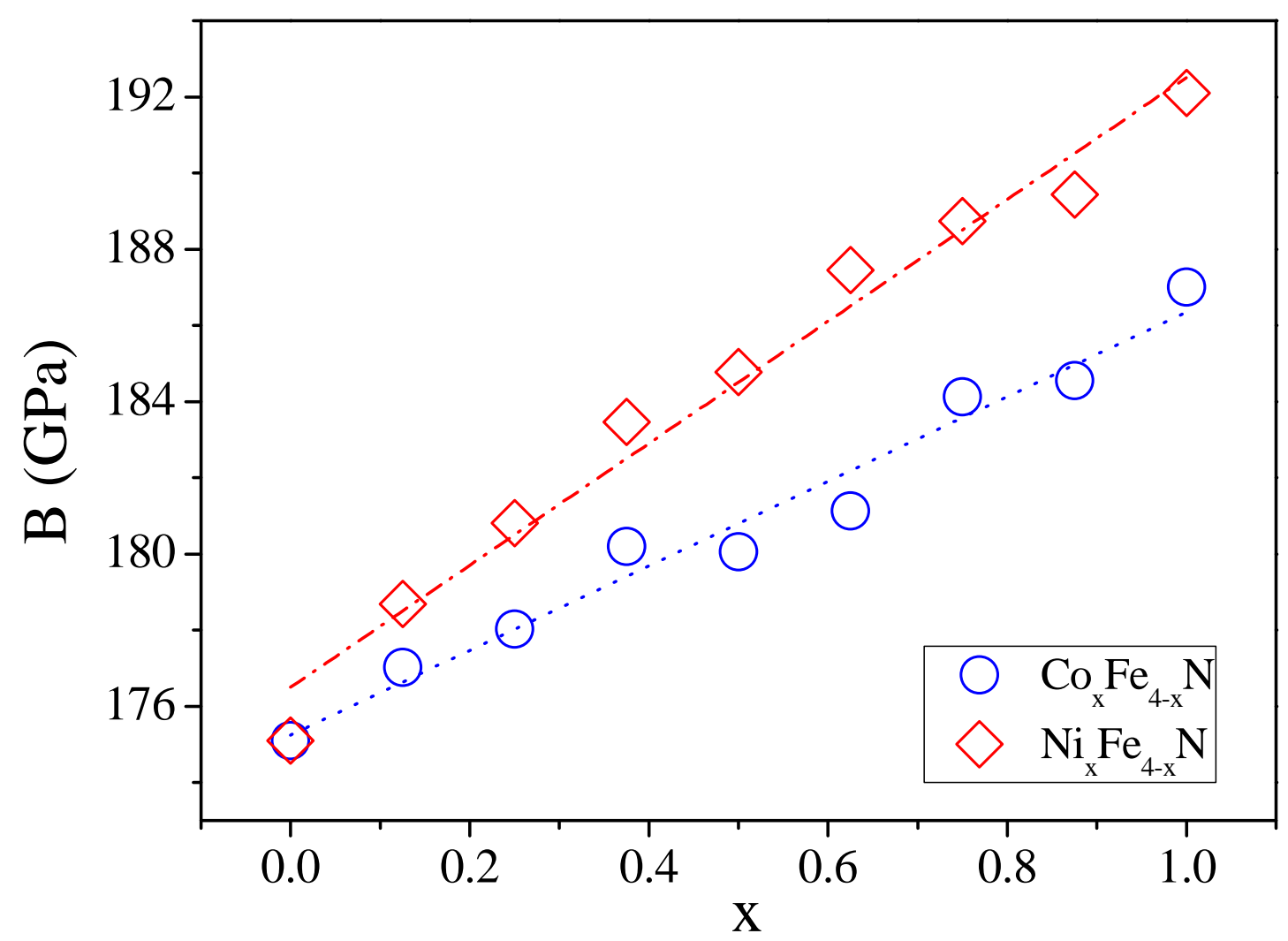

Figura 4.9: Dependencia lineal del módulo de mulk (B) con $x$ para los compuestos $\mathrm{Co}_{x} \mathrm{Fe}_{4-x} \mathrm{~N}_{\text {y Ni}} \mathrm{Fe}_{4-x} \mathrm{~N}$. 


\subsubsection{Propiedades Magnéticas e Hiperfinas}

La inclusión de átomos de Co o Ni produce una disminución del momento magnético total $\left(M_{f u}\right)$ del $\gamma^{\prime}-\mathrm{Fe}_{4} \mathrm{~N}$, tal como se puede observar en la Figura 4.10. Para ambos compuestos se observa una dependencia lineal decreciente de $M_{f u}$ respecto a $x$ con pendientes de $-0.79 \mu_{B} \mathrm{y}-1.86 \mu_{B}$ para los casos de $\mathrm{Co}_{x} \mathrm{Fe}_{4-x} \mathrm{~N}$ (Figura 4.10a) y $\mathrm{Ni}_{x} \mathrm{Fe}_{4-x} \mathrm{~N}$ (Figura 4.10b), respectivamente. Esto muestra una disminución de aproximadamente el $8 \%$ y el $19 \%$ del $M_{f u}$, respecto del $\gamma^{\prime}-\mathrm{Fe}_{4} \mathrm{~N}$, para los compuestos $\mathrm{CoFe}_{3} \mathrm{~N}$ y $\mathrm{NiFe}_{3} \mathrm{~N}$, respectivamente. A pesar de ello, el $M_{f u}$ de estos compuestos es mucho mayor que del $\alpha-\mathrm{Fe}(\mathrm{BCC})$. Para el caso de los compuestos $\mathrm{Ni}_{x} \mathrm{Fe}_{4-x} \mathrm{~N}$, fue posible corroborar con valores experimentales obtenidos de las referencias $[106,119,126]$ en conjunto con otros valores recopilados de $[104,105,107$, 109,120-122], observándose el mismo comportamiento lineal de $M_{f u}$ en función de $x$, con pendientes de $-2.0 \mu_{B}$ para los datos obtenidos de $[106,119,126]$. Esto indica una buena relación entre el valor teórico y los experimentales, aunque los valores teóricos sobreestiman los valores experimentales. Este hecho es conocido en virtud de la funcional utilizada GGA-PBE, la cual posee esta característica. La disminución de $M_{f u}$ con $x$, está relacionada con la ausencia de átomos de Fe (FeI) los cuales son sustituidos por átomos de Co o Ni. En la Figura 4.11 se muestran los valores del momento magnético de los átomos FeI y FeII en función de $x$ para los compuestos $\mathrm{Co}_{x} \mathrm{Fe}_{4-x} \mathrm{~N}$ y $\mathrm{Ni}_{x} \mathrm{Fe}_{4-x} \mathrm{~N}$, donde se observa que al sustituir un solo átomo de $\mathrm{FeI}$ por un $\mathrm{Co}$ o Ni en la supercelda de $\gamma^{\prime}-\mathrm{Fe}_{4} \mathrm{~N}$, se produce una disminución del momento magnético de los FeI y un pequeño aumento del momento magnético de los FeII, para luego mantenerse constante con valores de $2.83 \mu_{B}$ y $2.30 \mu_{B}$, respectivamente. Mientras que los átomos de Co y Ni muestran un momento magnético invariante con $x$, de $1.89 \mu_{B}$ y $0.83 \mu_{B}$ respectivamente. 


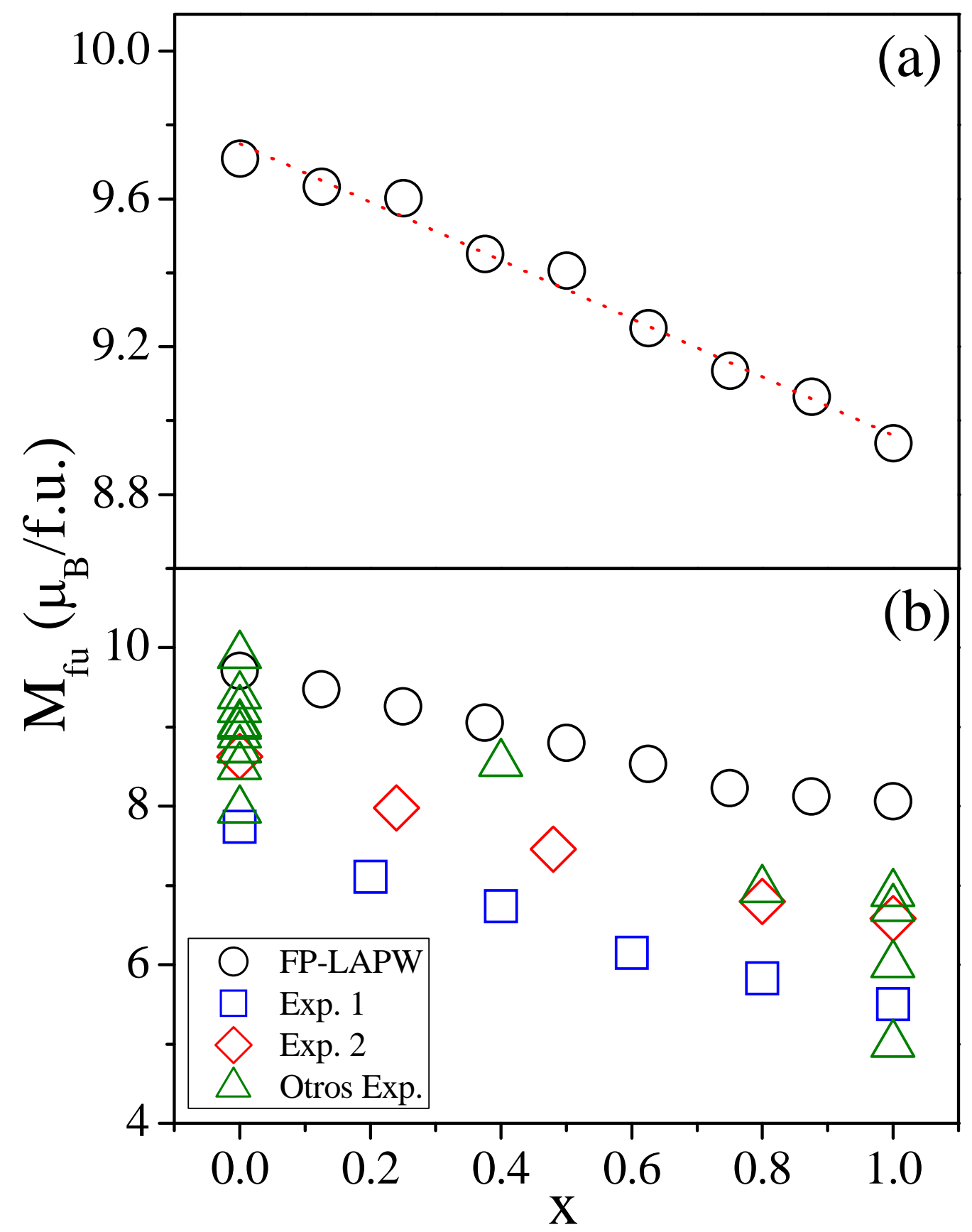

Figura 4.10: Dependencia del $M f u$ con $x$ para los compuestos a) $\mathrm{Co}_{x} \mathrm{Fe}_{4-x} \mathrm{~N}$ y b) $\mathrm{Ni}_{x} \mathrm{Fe}_{4-x} \mathrm{~N}$. En el caso b) se pudo comparar con los valores experimentales $[119,126]$ (cuadrados azules), [106] (rombos rojos) y otros valores experimentales recopilador [104,105, 107-109, 120-122] (triángulos verdes). 


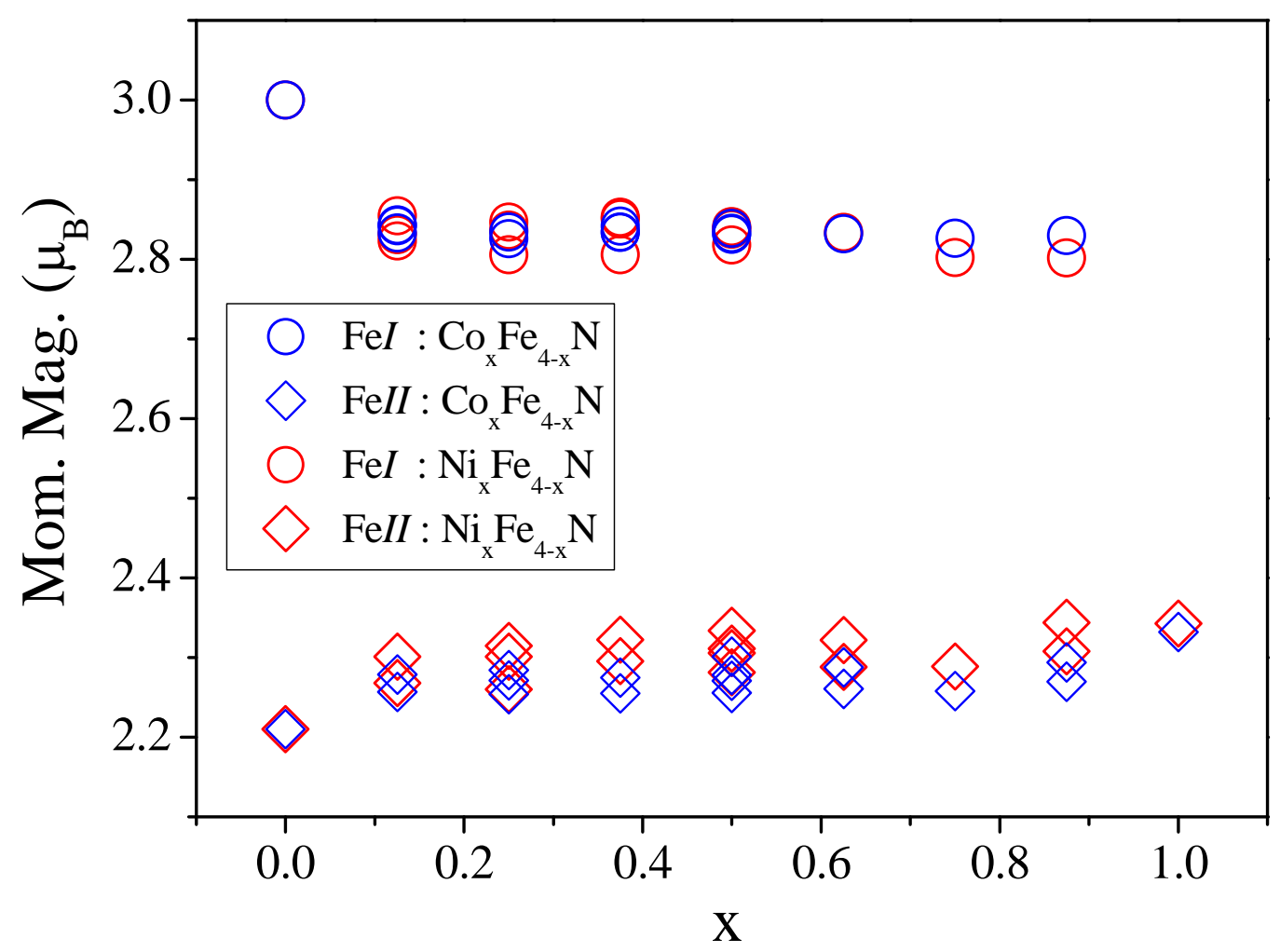

Figura 4.11: Valor del momento magnético de los átomos FeI y FeII de los compuestos $\mathrm{Co}_{x} \mathrm{Fe}_{4-x} \mathrm{~N}$ y $\mathrm{Ni}_{x} \mathrm{Fe}_{4-x} \mathrm{~N}$, para los diferentes valores de $x$.

Respecto de las propiedades hiperfinas, se determinó el campo hiperfino de contacto o de Fermi $\left(B_{h f}\right)$, el corrimiento isomérico $(\delta)$ y el desdoblamiento cuadrupolar $(\varepsilon)$ conforme se describió en el Capítulo 2. En los compuestos $\mathrm{Co}_{x} \mathrm{Fe}_{4-x} \mathrm{~N}$, el $B_{h f}$ de los FeI no varían con $x$, con un valor semejante al FeI del $\gamma^{\prime}-\mathrm{Fe}_{4} \mathrm{~N}$, mientras para los FeII, el $B_{h f}$ muestran una leve disminución conforme aumenta $x$ (Figura 4.12a), esto puede estar relacionado con la semejanza existente entre el átomo de Fe y el Co. 


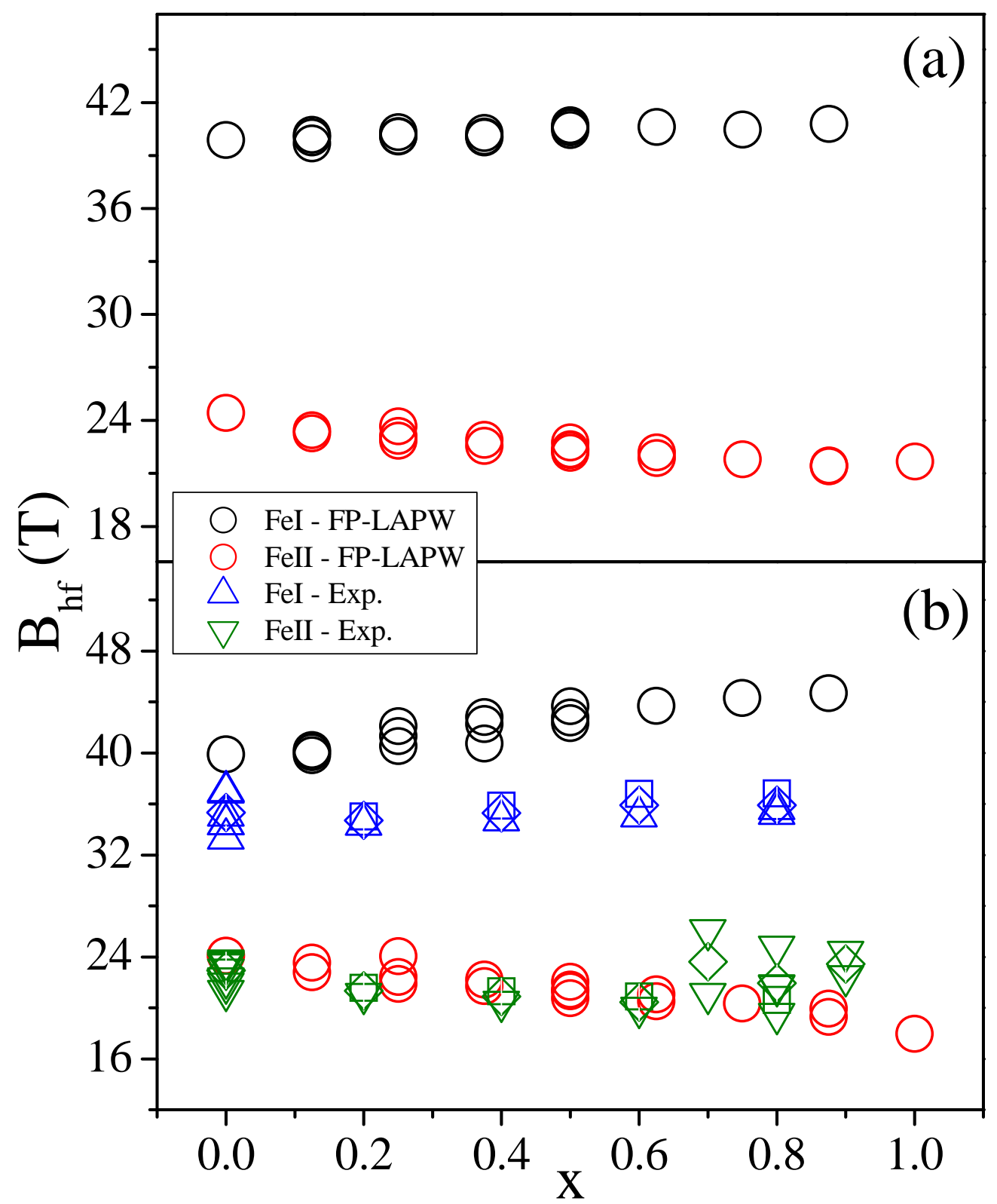

Figura 4.12: Variación del $B_{h f}$ con $x$ de los átomos FeI y FeII para los compuestos a) $\mathrm{Co}_{x} \mathrm{Fe}_{4-x} \mathrm{~N}$ y b) $\mathrm{Ni}_{x} \mathrm{Fe}_{4-x} \mathrm{~N}$, junto con diferentes valores experimentales recopilados de la bibliografía. Símbolos azules y verdes representan los valores de $B_{h f}$ experimental de los FeI y FeII, respectivamente. 
Para los compuestos $\mathrm{Ni}_{x} \mathrm{Fe}_{4-x} \mathrm{~N}$, el $B_{h f}$ de los FeI incrementan levemente con $x$ mientras que datos experimentales [104-107,109,119-122,126], muestran una invariancia de $B_{h f}$ con $x$. Lo opuesto ocurre para el $B_{h f}$ de los FeII, donde se observa una disminución lineal con $x$ (Figura 4.12b), estando esto en buena concordancia con los diferentes valores experimentales [104-109, 119-122,126]. El aumento y/o incremento del $B_{h f}$, está relacionado con la polarización de los orbitales $3 d-\mathrm{Fe}$ hacia los orbitales cercanos del núcleo, originando en estos últimos un desbalance en las distribuciones de carga up y down. La variación de $B_{h f}$ con $x$, de los átomos de $\mathrm{Fe}$, es más notoria en los compuestos de $\mathrm{Ni}_{x} \mathrm{Fe}_{4-x} \mathrm{~N}$.

En la figura 4.13 se muestra la dependencia del $\delta$ con $x$ para ambos compuestos. En el caso del $\mathrm{Co}_{x} \mathrm{Fe}_{4-x} \mathrm{~N}$, se observa que en los FeI el $\delta$ disminuye con $x$, mientras que para los FeII se mantiene un valor semejante al del $\gamma^{\prime}-\mathrm{Fe}_{4} \mathrm{~N}$ (Figura 4.13a). Esto muestra una mayor influencia de la concentración $x$ en la densidad de carga cercana al núcleo de los FeI, decreciendo a medida que aumenta $x$. Es distinto lo que ocurre en los compuestos $\mathrm{Ni}_{x} \mathrm{Fe}_{4-x} \mathrm{~N}$, en donde se observan leves variaciones del $\delta$ de los FeI, cuyo valor promedio es similar al FeI del $\gamma^{\prime}-\mathrm{Fe}_{4} \mathrm{~N}$. En el caso de los FeII, el $\delta$ muestra un leve incremento conforme aumenta el valor de $x$, para luego mantenerse casi constante (Figura 4.13b). En ambos tipos de Fe, se observa una buena correlación con los diferentes valores experimentales recopilados de la bibliografía [104-122,126]. El valor casi constantes del $\delta$ en los compuestos $\mathrm{Ni}_{x} \mathrm{Fe}_{4-x} \mathrm{~N}$, puede estar relacionada con la poca influencia magnética ejercida de los átomos de $\mathrm{Ni}$ hacia los átomos de $\mathrm{Fe}$, siendo esta una de las principales causa en la variación del $\delta$. 


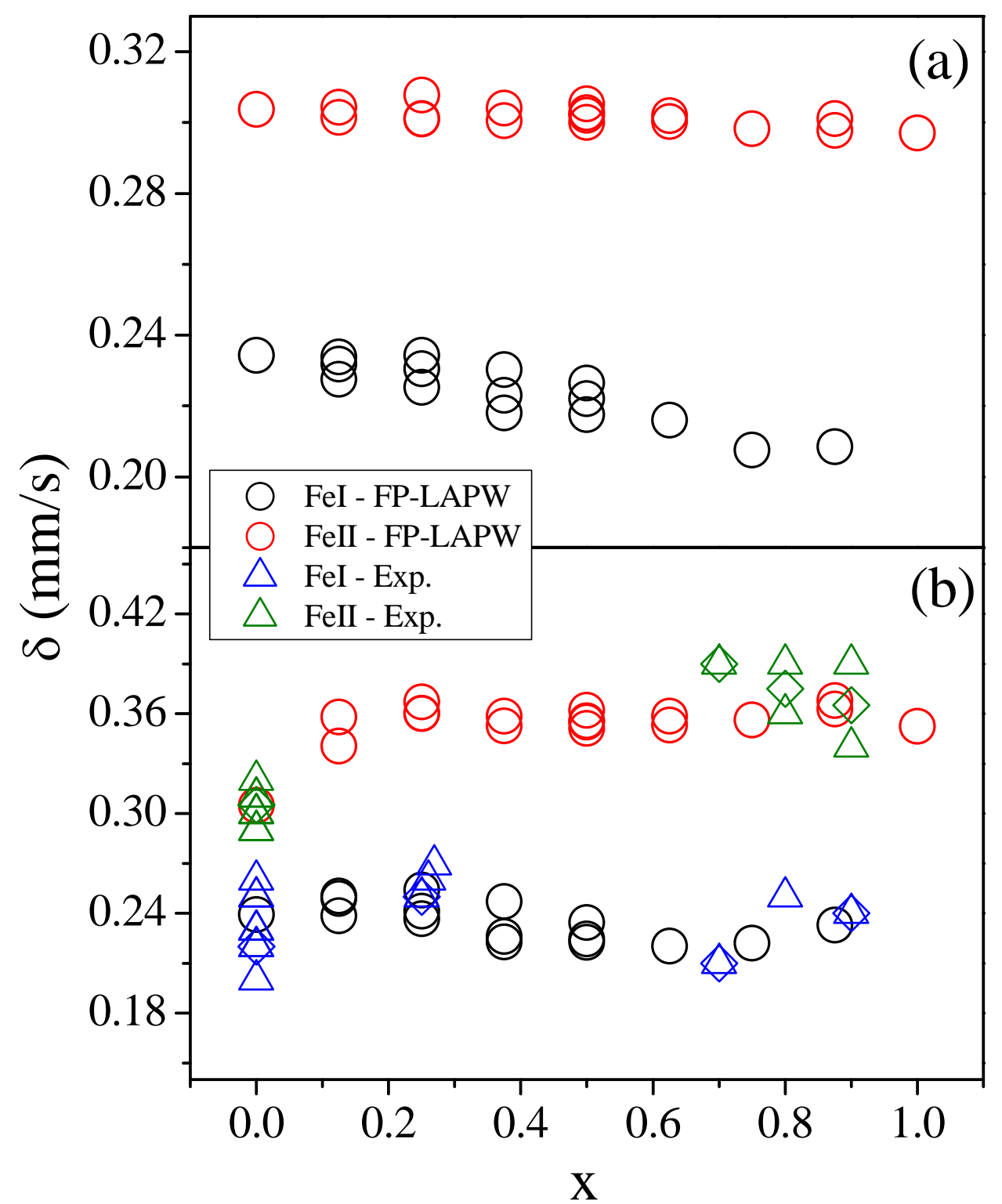

Figura 4.13: Variación del $\delta$ con $x$ de los átomos FeI y FeII para los compuestos a) $\mathrm{Co}_{x} \mathrm{Fe}_{4-x} \mathrm{~N}$ y b) $\mathrm{Ni}_{x} \mathrm{Fe}_{4-x} \mathrm{~N}$, junto con diferentes valores experimentales recopilados de la bibliografía. Símbolos azules y verdes representan los valores de $\delta$ experimental de los FeI y FeII, respectivamente. 
Finalmente, se analizará el $\varepsilon$ de los átomos Fe, para los FeI debido a su alta simetría cúbica del sitio cristalográfico presentan un valor cercano a cero, semejante al $\varepsilon$ del FeI en el compuesto $\gamma^{\prime}-\mathrm{Fe}_{4} \mathrm{~N}$. Mientras que para los FeII, el $\varepsilon$ aumenta linealmente con $x$ para ambos casos, con pendientes de $0.05 \mathrm{~mm} / \mathrm{s}$ y $0.16 \mathrm{~mm} / \mathrm{s}$ para los compuestos $\mathrm{Co}_{x} \mathrm{Fe}_{4-x} \mathrm{~N}_{\text {y Ni}} \mathrm{Fe}_{4-x} \mathrm{~N}$, respectivamente (Figura 4.14). El incremento del $\varepsilon$ está relacionado con la deformación de la simetría esférica de la distribución de carga cercana al núcleo, la cual pasa de una forma esférica a una elipsoidal con eje mayor paralelo al eje de magnetización. Dicha deformación es más intensa en los átomos FeII de los compuestos $\mathrm{Ni}_{x} \mathrm{Fe}_{4-x} \mathrm{~N}$. Este parámetro muestra la amplia influencia de los átomos Ni en la distribución de carga cercana al núcleo de los FeII, como consecuencia de modificar su entorno, esto es, al sustituir átomos de FeI por átomos de Co o Ni modifica el entorno, ubicados como segundos vecinos ( $n n n)$, de los átomos de FelI.

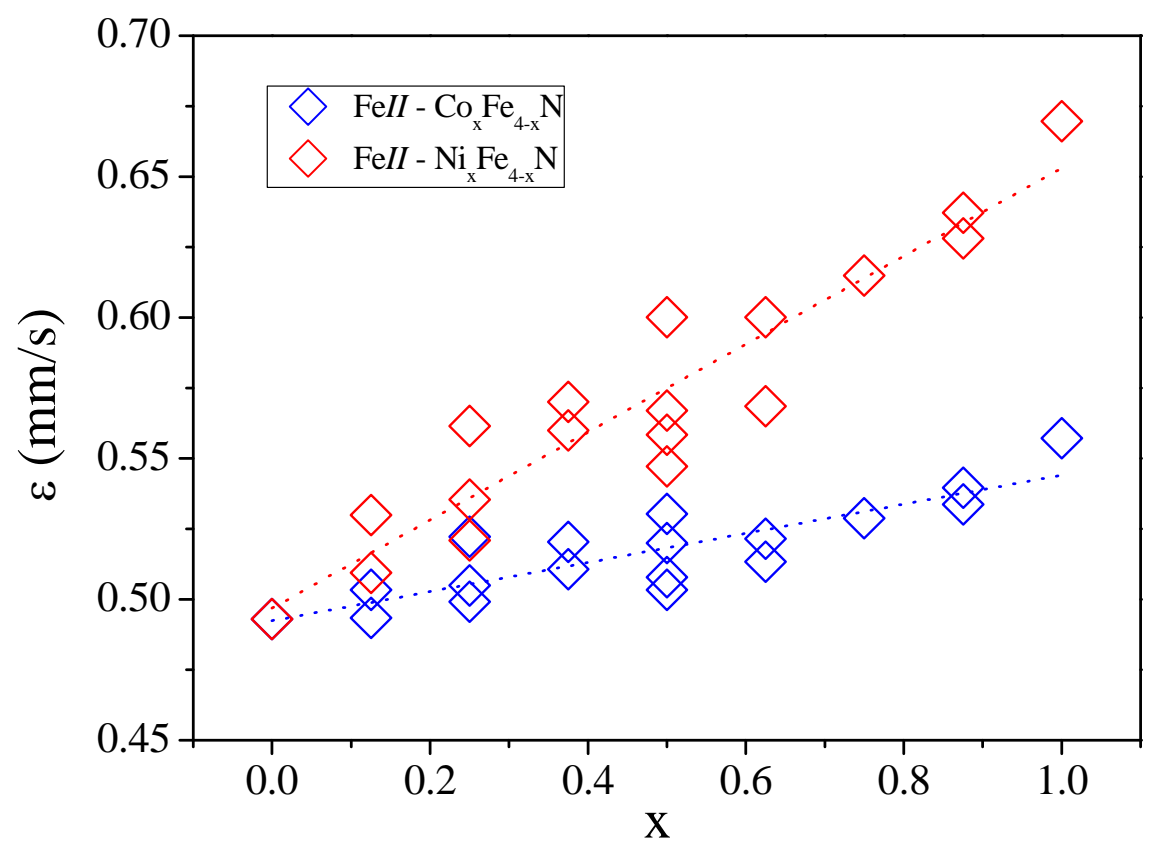

Figura 4.14: Dependencia lineal del $\varepsilon$ con $x$ de los átomos FelI para los compuestos a) $\mathrm{Co}_{x} \mathrm{Fe}_{4-x} \mathrm{~N}$ y b) $\mathrm{Ni}_{x} \mathrm{Fe}_{4-x} \mathrm{~N}$. 


\subsection{Conclusiones}

El presente Capítulo se dividió en dos partes principales, la primera fue dedicada para el estudio de los compuestos ternarios $\mathrm{Mn}_{x} \mathrm{Fe}_{4-x} \mathrm{~N}$, lográndose determinar el sitio cristalográfico preferencial de sustitución del Mn en el compuesto $\gamma^{\prime}-\mathrm{Fe}_{4} \mathrm{~N}$, siendo este el lugar ocupado por el FeII, con un parámetro de red semejante al del $\gamma^{\prime}-\mathrm{Fe}_{4} \mathrm{~N}$. Simultáneamente se determinó el estado fundamental magnético de estos compuestos. Llegando a la conclusión que la configuración antiparalela de los momentos magnéticos de los Fe y Mn es la de mínima energía, pero con un $M_{f u}$ resultante distinto a cero, caracterizando a estos compuestos como ferrimagnetos. Todo esto ayudó a explicar el único trabajo experimental realizado, a muy bajas concentraciones de $\mathrm{Mn}$, que existe de este compuesto, en donde no fue posible distinguir el sitio preferencial de sustitución.Lo cual concuerda con los cálculos realizados, en donde se encontró que a bajas concentraciones la sustitución en el sitio cristalográfico del FeI o FeII con alineaciones paralela o antiparalela de los momentos magnéticos de los Fe y Mn, poseen casi el mismo valor energético. Pudiéndose recién distinguir dichas configuraciones conforme aumenta la concentración de Mn.

Concerniente a las propiedades magnéticas, el hecho que los átomos de $\mathrm{Mn}$ tengan un momento magnético alineado de forma antiparalela a la de los Fe, produce una reducción del momento magnético de los Fe, ocasionando así una notable disminución del $M_{f u}$. Las variaciones del momento magnético de los Fe se ven reflejadas en sus respectivos parámetros hiperfinos como el campo hiperfino, el corrimiento isomérico y el desdoblamiento cuadrupolar. Siendo más notoria la fuerte dependencia del campo hiperfino con $x$, en donde se observa un comportamiento semejante a la del momento magnético. La dependencia entre el momento magnético por átomo y su respectivo $B_{h f}$ fue posible cuantificarla determinando la denominada constante de acoplamiento hiperfino $(A)$.

La segunda parte del presente Capítulo fue dedicada al estudio simultáneo de los compuestos ternarios $\mathrm{Co}_{x} \mathrm{Fe}_{4-x} \mathrm{~N}_{\text {y Ni}} \mathrm{Ni}_{4} \mathrm{Fe}_{4} \mathrm{~N}$, en donde se consideró que los átomos de Co o Ni sustituyen a los FeI, siendo este el sitio de sustitución prefe- 
rencial conforme fue determinado en el Capítulo 2. Para el caso de los compuestos $\mathrm{Co}_{x} \mathrm{Fe}_{4-x} \mathrm{~N}$, las propiedades calculadas no han sido posibles compararlas con datos experimentales, permitiéndonos predecirlas teóricamente, basándonos en la buena comparación existente de dichas propiedades calculadas y las experimentales para el caso de los compuestos $\mathrm{Ni}_{x} \mathrm{Fe}_{4-x} \mathrm{~N}$. 


\section{Capítulo 5}

\section{Estudio de los Compuestos}

\section{Ternarios $\boldsymbol{M}_{x} \mathbf{F e}_{4-x} \mathbf{N}(M=\mathbf{A l}, \mathbf{G a}$,}

In)

En el presente capítulo se estudiará la variación de las propiedades estructurales, electrónicas, magnéticas e hiperfinas de los compuestos $M_{x} \mathrm{Fe}_{4-x} \mathrm{~N}$ en función de la concentración $(x)$ del átomo $M(M=\mathrm{Ga}, \mathrm{Al}, \mathrm{In})$ para $0 \leq x \leq 1$. La primera parte estará dedicada al estudio de la estabilidad estructural y magnética de los compuestos $\mathrm{MFe}_{3} \mathrm{~N}(\mathrm{M}=\mathrm{Ga}, \mathrm{Al}, \mathrm{In})$. Seguidamente se estudiará la dependencia con $x$ de las propiedades estructurales, magnéticas e hiperfinas de los compuestos $M_{x} \mathrm{Fe}_{4-x} \mathrm{~N}$ para $0 \leq x \leq 1$.

\subsection{Estado Fundamental de los Compuestos $M \mathrm{Fe}_{3} \mathrm{~N}$ ( $M=\mathbf{A} \mathbf{l}, \mathbf{G a}, \mathbf{I n})$}

Con el fin de determinar el sitio de sustitución preferencial del átomo $M$ en el $\gamma^{\prime}-\mathrm{Fe}_{4} \mathrm{~N}$, se obtuvo la curva energía total - volumen para los casos en que el átomo $M$ sustituye a un FeI o un FeII, estructuras $P m \overline{3} m$ y $P 4 / m m m$, respectivamente. De la Figura 5.1 se puede observar que la configuración de mínima energía es la $P m \overline{3} m$, esto muestra que el sitio preferencial de sustitución del átomo $M$ es el del 
$\mathrm{FeI}$, siendo los compuestos ternarios $M \mathrm{Fe}_{3} \mathrm{~N}$ isoestructurales al $\gamma^{\prime}-\mathrm{Fe}_{4} \mathrm{~N}$. Con el propósito de determinar el parámetro de red de equilibrio de estos compuestos con estructura $\operatorname{Pm} \overline{3} m$, los datos de energía total - volumen fueron ajustados con la ecuación de estado de Birch-Murnaghan (2.2). En la Tabla 5.1, se reportan los valores del parámetro de red de equilibrio $(a)$ como también el respectivo momento magnético total $\left(M_{f u}\right)$ y por átomo $(m)$ de cada compuesto, además de los valores experimentales (extrapolados a $x=1$ de los compuestos $M_{x} \mathrm{Fe}_{4-x} \mathrm{~N}$ ) y teóricos obtenidos de la bibliografía. 


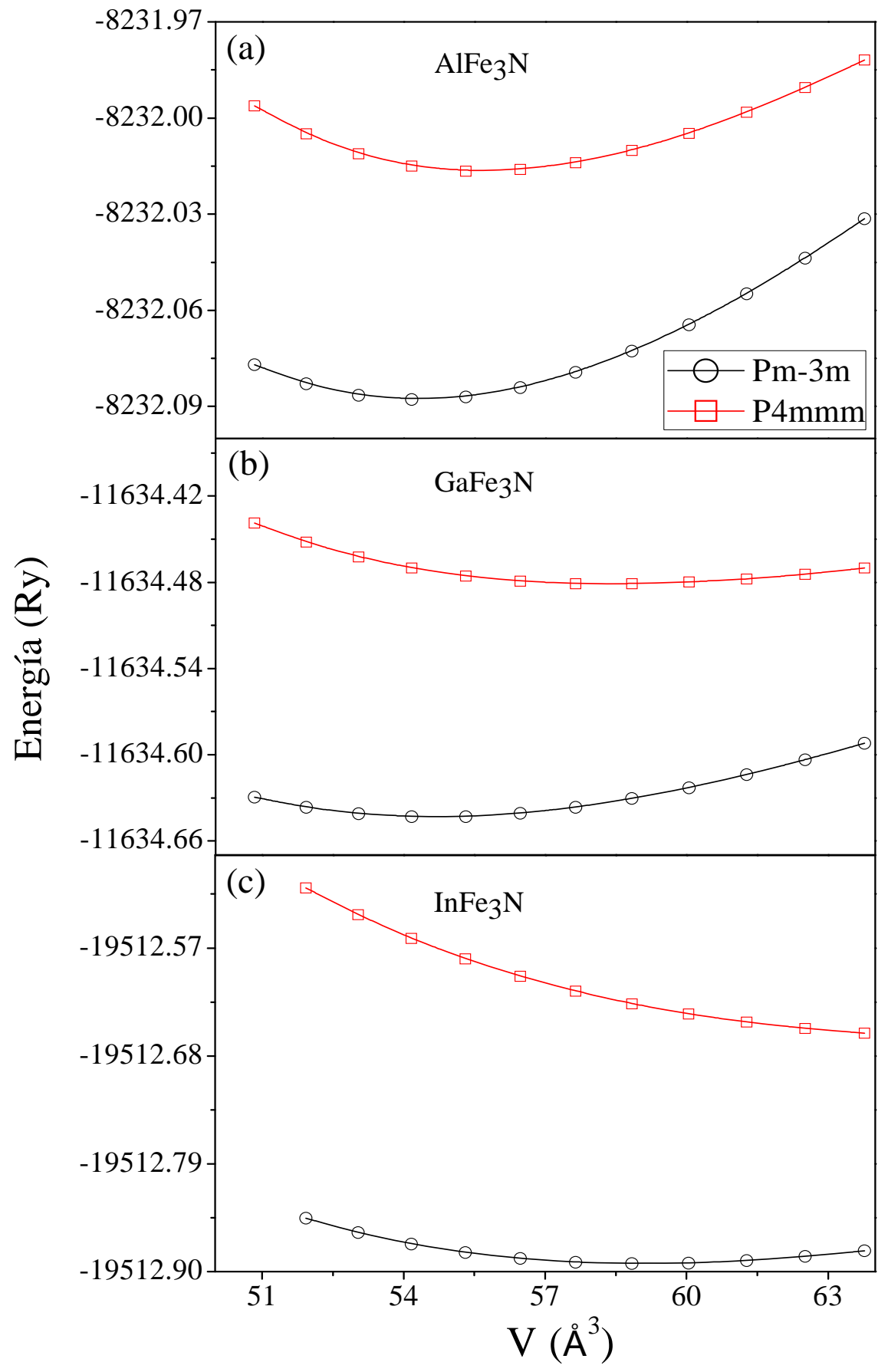

Figura 5.1: Curvas energía total - volumen de las estructuras $P m \overline{3} m$ y $P 4 / m m m$ de los compuestos a) $\left.\mathrm{AlFe}_{3} \mathrm{~N}, \mathrm{~b}\right) \mathrm{GaFe}_{3} \mathrm{~N}$ y c) $\mathrm{InFe}_{3} \mathrm{~N}$. 
En la misma tabla se muestran también los valores de a calculados junto con otros valores teóricos y experimentales obtenidos de la bibliografía. Para el $\mathrm{AlFe}_{3} \mathrm{~N}$, se observa una buena concordancia tanto con los valores experimentales $[155,191]$ como teóricos [192-195] de otros autores, mientras que para el $\mathrm{GaFe}_{3} \mathrm{~N}$ se encontró una buena relación entre el $a$ calculado y el experimental $[149,150]$, pero difiere levemente con el valor teórico publicado en [149]. En el caso del $\operatorname{InFe}_{3} \mathrm{~N}$, el $a$ calculado es menor que el teórico obtenido en [113], pero más cercano a los valores experimentales publicados en $[113,152]$. Respecto al $M_{f u}$, los valores obtenidos difieren considerablemente de los valores experimentales (Tabla 5.1), los cuales caracterizan a estos materiales como antiferromagnetos con un valor de $M_{f u}$ cercano a cero $[113,115,149,191]$, mientras que los resultados teóricos reportan valores de $M_{f u}$ distintos de cero $[113,156]$.

Tabla 5.1: Valores de $a, M_{f u}$ y momento magnético por átomo $(m)$ obtenidos de los cálculos ab-initio junto con otros valores teóricos y experimentales.

\begin{tabular}{|c|c|c|c|c|}
\hline & & $\mathrm{AlFe}_{3} \mathrm{~N}$ & $\mathrm{GaFe}_{3} \mathrm{~N}$ & $\mathrm{InFe}_{3} \mathrm{~N}$ \\
\hline \multirow[t]{7}{*}{$a(\AA)$} & \multirow{4}{*}{ Teórico } & 3.7857 & 3.7977 & 3.8945 \\
\hline & & 3.7984 [192] & 3.7900 [149] & 3.9107 [113] \\
\hline & & 3.7715 [192] & & \\
\hline & & $3.7677[193,194]$ & & \\
\hline & \multirow{3}{*}{ Experimental } & 3.7150 [195] & & \\
\hline & & 3.7967 [155] & 3.7974 [191] & 3.8673 [113] \\
\hline & & 3.8026 [191] & 3.8001 [149] & 3.8788 [152] \\
\hline \multirow[t]{4}{*}{$M_{f u}\left(\mu_{B} / f u\right)$} & & 5.14 & 5.30 & 5.48 \\
\hline & Teórico & & 5.00 [149] & 5.50 [152] \\
\hline & Experimental & 5.35 [191] & 0.00 [191] & 0.00 [152] \\
\hline & & & $5.40[150]$ & \\
\hline \multirow[t]{2}{*}{$m\left(\mu_{B}\right)$} & FeII & 1.73 & 1.78 & 1.82 \\
\hline & $M$ & -0.14 & -0.11 & -0.11 \\
\hline
\end{tabular}

Con el propósito de determinar el estado fundamental magnético de cada compuesto, se realizaron cálculos mediante la técnica fixed spin moment, (FSM, mo- 
mento magnético fijo) en donde se estableció un valor de $M_{f u}$ y se varió el valor del parámetro de red, pudiéndose encontrar así el parámetro de red de equilibrio (a) para dicho valor de $M_{f u}$. Este procedimiento se realizó para diferentes valores de $M_{f u}$, determinándose así el $a$ y su respectiva energía total $(E)$ para cada valor de $M_{f u}$. En la Figura 5.2 se muestra la variación de la energía total $\Delta E=E\left(M_{f u}\right)-E(0)$, respecto al estado $M_{f u}=0$, en función de $M_{f u}$, apreciándose la existencia de barreras de energía entre el estado $M_{f u}=0$ (A) y el estado ferrimagnético (B). Para todos los casos se observa que la barrera de energía es asimétrica, esto es, la barrera de energía de A hacia B es distinta a la de B hacia A (Tabla 5.2), donde el estado B pertenece a un mínimo global. Este mínimo es menos notorio para el $\mathrm{AlFe}_{3} \mathrm{~N}$ y más notoria para el $\mathrm{GaFe}_{3} \mathrm{~N}$ y el $\mathrm{InFe}_{3} \mathrm{~N}$, mostrando así la estabilidad energética del estado magnético de estos compuestos. Para el caso del $\mathrm{GaFe}_{3} \mathrm{~N}$, medidas a diferentes temperaturas del $\mathrm{Ga}_{0.9} \mathrm{Fe}_{3.1} \mathrm{~N}$ reportadas en [150], muestran que a baja temperatura el compuesto manifiesta una magnetización cercana a los $5 \mu_{B}$, corroborando así los valores teóricos obtenidos en el presente trabajo teniendo en cuenta que la DFT es una teoría desarrollada a $0 \mathrm{~K}$.

Tabla 5.2: Valores de las barreras de energía (mRy) del punto A hacia $\mathrm{B}(\mathrm{A} \rightarrow \mathrm{B})$ y viceversa $(\mathrm{B} \rightarrow \mathrm{A})$.

\begin{tabular}{cccc}
\hline & $\mathrm{AlFe}_{3} \mathrm{~N}$ & $\mathrm{GaFe}_{3} \mathrm{~N}$ & $\mathrm{InFe}_{3} \mathrm{~N}$ \\
\hline $\mathrm{A} \rightarrow \mathrm{B}$ & 3.654 & 1.658 & 0.174 \\
$\mathrm{~B} \rightarrow \mathrm{A}$ & 4.331 & 13.748 & 28.974 \\
\hline
\end{tabular}




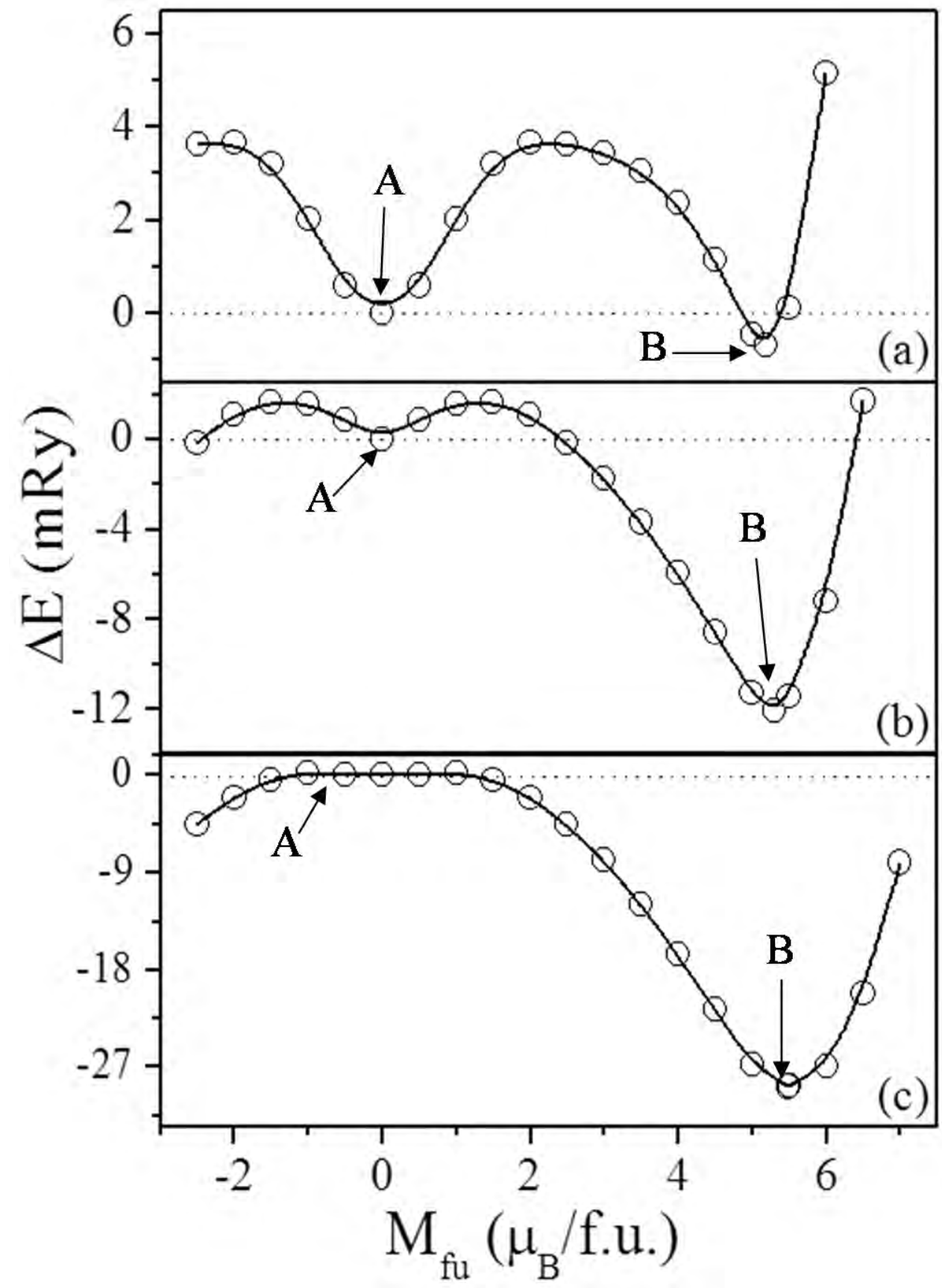

Figura 5.2: Variación de $\Delta E$ en función del $M_{f u}$ para a) $\mathrm{AlFe}_{3} \mathrm{~N}$, b) $\mathrm{GaFe}_{3} \mathrm{~N}$ y c) $\operatorname{InFe}_{3} \mathrm{~N}$. 
En la Figura 5.3, se muestra la variación del parámetro de red de equilibrio $(a)$ en función del $M_{f u}$, donde se observa un incremento parabólico de $a$ con $M_{f u}$. Esto indica la presencia del efecto magneto-volumétrico en estos compuestos, que puede ser caracterizado por medio de la constante de acoplamiento magneto-volumétrica $\left(\kappa C_{m v}\right)$, la cual está asociada con el incremento del volumen relativo $(\omega)$ y el momento magnético total $\left(M_{f u}\right)$ del compuesto [196]:

$$
\omega=\frac{V\left(M_{f u}\right)-V(0)}{V(0)}=\kappa C_{m v} M_{f u}^{2}
$$

Obteniéndose así los valores de $2.02 \times 10^{-3}, 1.92 \times 10^{-3}$ y $1.76 \times 10^{-3} \mu_{B}^{-2}$ para el $\mathrm{AlFe}_{3} \mathrm{~N}, \mathrm{GaFe}_{3} \mathrm{~N}$ y $\operatorname{InFe}_{3} \mathrm{~N}$, respectivamente, los cuales son cercanos al del $\alpha-\mathrm{Fe}$ [196] y característicos de aleaciones $3 d$ [196]. El valor de $\kappa C_{m v}$ nos indica una gran dependencia entre el volumen de la estructura cristalina y el momento magnético de estos compuestos ternarios, cuyos parámetros de red son semejantes a los valores experimentales.

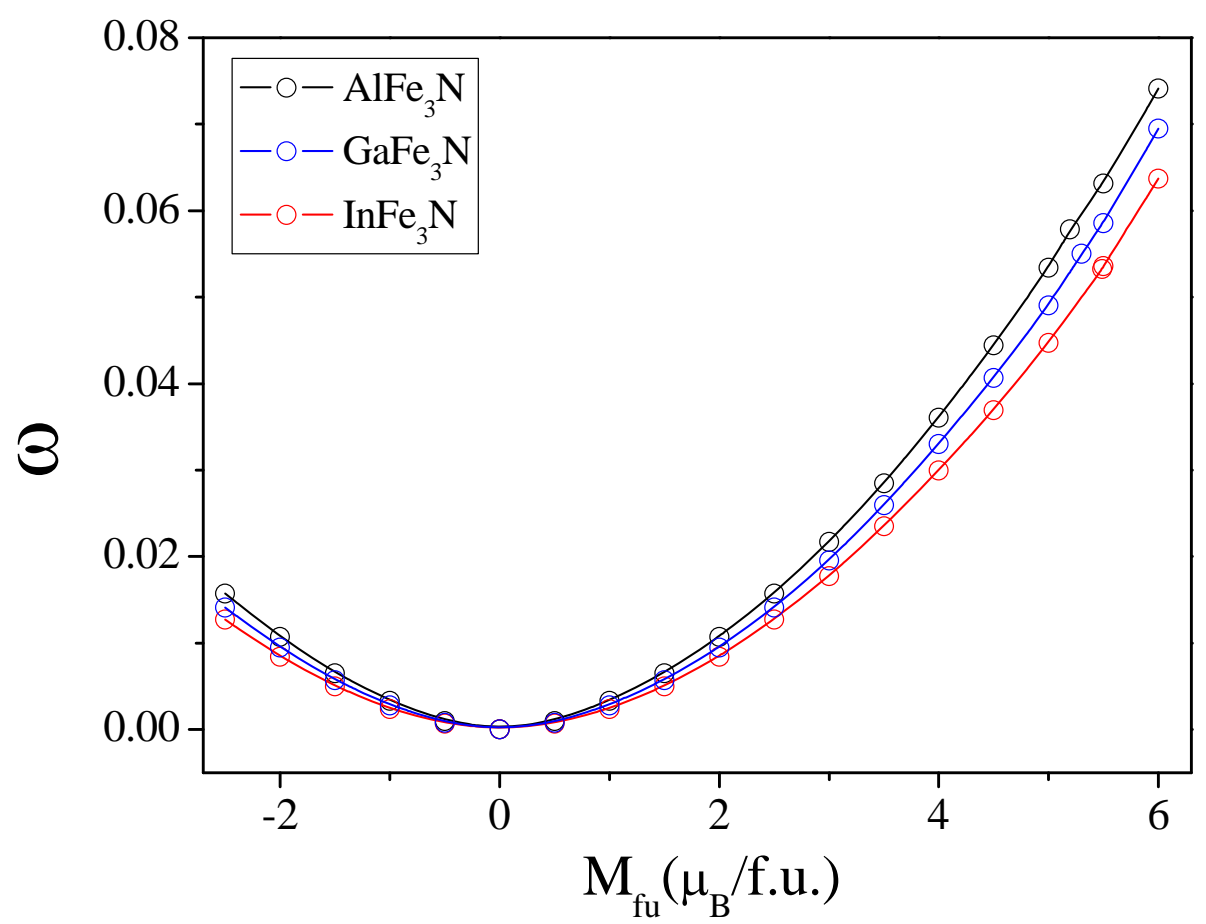

Figura 5.3: Variación del volumen relativo $\omega$ respecto de $M_{f u}$ para los compuestos $\mathrm{AlFe}_{3} \mathrm{~N}$, $\mathrm{GaFe}_{3} \mathrm{~N}$ y $\mathrm{InFe}_{3} \mathrm{~N}$. 


\subsection{Compuestos Ternarios $M_{x} \mathbf{F} \mathbf{e}_{4-x} \mathbf{N}(M=\mathrm{Al}, \mathrm{Ga}$, In)}

Habiendo determinado el estado fundamental estructural y magnético de los compuestos $M \mathrm{Fe}_{3} \mathrm{~N}$, se estudiará la evolución con $x$ del parámetro de red y de las propiedades magnéticas e hiperfinas de los compuestos $M_{x} \mathrm{Fe}_{4-x} \mathrm{~N}(M=\mathrm{Al}, \mathrm{Ga}$, In) para $0 \leq x \leq 1$, siendo necesario el uso de una supercelda de $2 \times 2 \times 2$ de $\gamma^{\prime}-\mathrm{Fe}_{4} \mathrm{~N}$. Para cada valor de $x$ se han considerado todas las configuraciones posibles como se describen en el Capítulo 2. En todos los casos se ha considerado que el átomo $M$ sustituye a un átomo FeI, es decir ocupan los sitios $1 a$ de Wyckoff.

\subsubsection{Propiedades Estructurales}

Para los compuestos $M_{x} \mathrm{Fe}_{4-x} \mathrm{~N}(M=\mathrm{Al}, \mathrm{Ga}$, In) se determinaron las curvas energía total - volumen para todas las configuraciones posibles de cada valor de $x$. En la Tabla 5.3, se muestran las configuraciones de mínima energía para cada valor de $x$ correspondiente a cada compuesto ternario, dichas configuraciones se usarán para el cálculo de las propiedades estructurales, magnéticas e hiperfinas de cada compuesto. En la Tabla 5.4 se reportan los respectivos parámetros de red de equilibrio (a) de cada compuesto, obtenido del ajuste de los datos de energía total - volumen con la ecuación de estado de Birch-Murnaghan (2.2), como también los respectivos valores del módulo de bulk (B).

Tabla 5.3: Configuraciones de mínima energía correspondiente a cada valor de $x$.

\begin{tabular}{cccccccc}
\hline$x$ & 0.125 & 0.250 & 0.375 & 0.500 & 0.625 & 0.750 & 0.875 \\
\hline $\mathrm{Al}_{x} \mathrm{Fe}_{4-x} \mathrm{~N}$ & $\mathrm{C}_{1}^{1}$ & $\mathrm{C}_{12}^{2}$ & $\mathrm{C}_{123}^{3}$ & $\mathrm{C}_{1237}^{4}$ & $\mathrm{C}_{12345}^{5}$ & $\mathrm{C}_{123456}^{6}$ & $\mathrm{C}_{2345678}^{7}$ \\
$\mathrm{Ga}_{x} \mathrm{Fe}_{4-x} \mathrm{~N}$ & $\mathrm{C}_{1}^{1}$ & $\mathrm{C}_{18}^{2}$ & $\mathrm{C}_{258}^{3}$ & $\mathrm{C}_{1347}^{4}$ & $\mathrm{C}_{12345}^{5}$ & $\mathrm{C}_{123456}^{6}$ & $\mathrm{C}_{2345678}^{7}$ \\
$\mathrm{In}_{x} \mathrm{Fe}_{4-x} \mathrm{~N}$ & $\mathrm{C}_{1}^{1}$ & $\mathrm{C}_{12}^{2}$ & $\mathrm{C}_{123}^{3}$ & $\mathrm{C}_{1237}^{4}$ & $\mathrm{C}_{12345}^{5}$ & $\mathrm{C}_{123456}^{6}$ & $\mathrm{C}_{2345678}^{7}$ \\
\hline
\end{tabular}


Tabla 5.4: Configuraciones de mínima energía correspondiente a cada valor de $x$.

\begin{tabular}{ccccccccc}
\hline & \multicolumn{8}{c}{$a(\AA)$} \\
$x$ & 0.125 & 0.250 & 0.375 & 0.500 & 0.625 & 0.750 & 0.875 & 1.000 \\
\hline $\mathrm{Al}_{x} \mathrm{Fe}_{4-x} \mathrm{~N}$ & 3.7918 & 3.7882 & 3.7861 & 3.7825 & 3.7782 & 3.7790 & 3.7806 & 3.7857 \\
$\mathrm{Ga}_{x} \mathrm{Fe}_{4-x} \mathrm{~N}$ & 3.7912 & 3.7918 & 3.7919 & 3.7929 & 3.7933 & 3.7940 & 3.7953 & 3.7977 \\
$\mathrm{In}_{x} \mathrm{Fe}_{4-x} \mathrm{~N}$ & 3.8064 & 3.8172 & 3.8333 & 3.8414 & 3.8565 & 3.8678 & 3.8804 & 3.8945 \\
\hline & & & \multicolumn{7}{c}{$\mathrm{B}(\mathrm{GPa})$} \\
$x$ & 0.125 & 0.250 & 0.375 & 0.500 & 0.625 & 0.750 & 0.875 & 1.000 \\
\hline $\mathrm{Al}_{x} \mathrm{Fe}_{4-x} \mathrm{~N}$ & 184 & 188 & 152 & 183 & 159 & 162 & 150 & 197 \\
$\mathrm{Ga}_{x} \mathrm{Fe}_{4-x} \mathrm{~N}$ & 206 & 205 & 204 & 197 & 180 & 167 & 177 & 217 \\
$\mathrm{In}_{x} \mathrm{Fe}_{4-x} \mathrm{~N}$ & 183 & 185 & 187 & 194 & 192 & 195 & 197 & 202 \\
\hline
\end{tabular}

En la Figura 5.4, se muestra la dependencia de $a$ con $x$ de los compuestos $\mathrm{Al}_{x} \mathrm{Fe}_{4-x} \mathrm{~N}, \mathrm{Ga}_{x} \mathrm{Fe}_{4-x} \mathrm{~N}, \mathrm{In}_{x} \mathrm{Fe}_{4-x} \mathrm{~N}$. Para el caso del $\mathrm{Al}_{x} \mathrm{Fe}_{4-x} \mathrm{~N}$ (Figura 5.4a), se observa un pequeño aumento de $a$ con $x$, para luego decrecer de forma casi lineal hasta $x=0.625$, donde empieza a aumentar nuevamente. Este comportamiento no lineal de $a$ con $x$ puede estar relacionado con la diferencia entre los radios atómicos del Fe $(1.56 \AA)$ y del $\mathrm{Al}(1.18 \AA)$. Esta dependencia no lineal decreciente de $a$ con $x$, es característico de compuestos denominados not completely normal or inverse (no completamente normales o inversos) [182-184]. Lamentablemente no existen datos experimentales de los compuestos $\mathrm{Al}_{x} \mathrm{Fe}_{4-x} \mathrm{~N}$ con los cuales se pueda establecer comparación.

Para el caso de los compuestos $\mathrm{Ga}_{x} \mathrm{Fe}_{4-x} \mathrm{~N}$, el $a$ aumenta de forma no lineal con $x$ (Figura 5.4b). Este comportamiento de $a$ con $x$ está en conformidad con los valores experimentales de [155], donde realizaron mediciones de Rayos X usando radiación $\mathrm{Cu}-\mathrm{K} \alpha_{1}(\lambda=1.54059 \AA)$. Los mismos autores de [155], posteriormente publicaron mediciones de Rayos $\mathrm{X}$ de los mismos compuestos pero usando radiación Mo-K $\alpha_{1}$ $(\lambda=0.70932 \AA)$ [149] reportando una dependencia de $a$ con $x$ totalmente distinta al publicado en [155] a pesar de haber usado las misma técnicas de preparación de las muestras. Para el posterior análisis de las propiedades de los compuestos $\mathrm{Ga}_{x} \mathrm{Fe}_{4-x} \mathrm{~N}$, se usaran los datos experimentales de [155], debido a que para el caso 
del $\gamma^{\prime}-\mathrm{Fe}_{4} \mathrm{~N}$ el valor de $a$ es semejante a las medidas experimentales usando ya sea $\mathrm{Cu}-\mathrm{K} \alpha_{1}[77,151]$ o Mo-K $\alpha_{1}[49,50]$ (Tabla 2.1), mientras que en [149] sobrestiman el valor de $a$ del $\gamma^{\prime}-\mathrm{Fe}_{4} \mathrm{~N}$ a pesar de haber usado la misma radiación que en $[49,50]$, siendo esta última la más aceptada en la literatura. Además, los valores experimentales de $a$ en [155] se asemejan a los valores calculados teóricamente. La dependencia no lineal de $a$ con $x$ está relacionada a una doble contribución. 


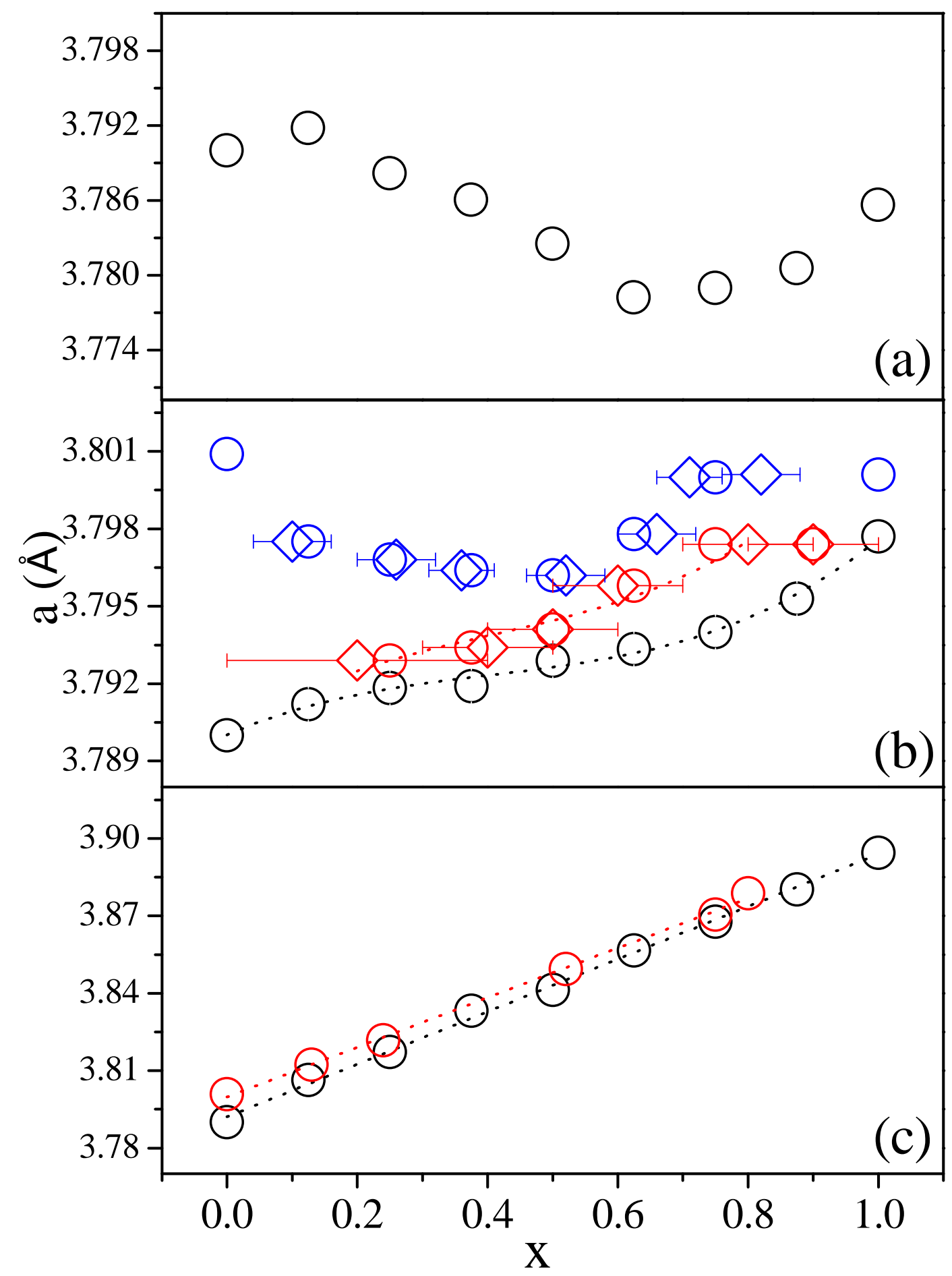

Figura 5.4: Variación del parámetro de red $(a)$ con $x$ (círculos negros) para los compuestos a) $\mathrm{Al}_{x} \mathrm{Fe}_{4-x} \mathrm{~N}$, b) $\mathrm{Ga}_{x} \mathrm{Fe}_{4-x} \mathrm{~N}$, junto a los valores experimentales de [155] (símbolos rojos) y [149] (símbolos azules), c) $\operatorname{In}_{x} \mathrm{Fe}_{4-x} \mathrm{~N}$, junto a los valores experimentales de [152] (símbolos rojos). 
Por un lado está la diferencia de los radios metálicos existente entre el átomo de Fe y de Ga $(1.24 \AA$ y $1.41 \AA$, respectivamente [190]), y por otro lado está el tipo de interacción magnética existente entre dichos átomos. Considerando que los sistemas no son soluciones sólidas aleatorias, tanto los datos teóricos y los experimentales [155], se ajustaron con la ecuación de Vegard modificada de tercer orden [197], obteniéndose así los polinomios:

$$
\begin{aligned}
a^{\text {cal }}(x)= & 3.7900_{2}(1-x)+3.7976_{2} x+0.0040_{2} x(1-x)^{2} \\
& -0.0130_{2} x^{2}(1-x) \\
a^{e x p}(x)= & 3.7900_{2}(1-x)+3.8020_{3} x+0.0051_{5} x(1-x)^{2} \\
& -0.0175_{6} x^{2}(1-x)
\end{aligned}
$$

De las ecuaciones (5.1) y (5.2) se puede observar una buena correlación entre el ajuste de los datos experimentales y los teóricos, considerando además que las medidas experimentales están realizadas a temperatura ambiente mientras que los cálculos representan los valores a $0 \mathrm{~K}$.

En la Figura 5.4c, se muestra la variación de $a$ con $x$ para los compuestos $\operatorname{In}_{x} \mathrm{Fe}_{4-x} \mathrm{~N}$, allí se ve un incremento lineal de a con la concentración de In. A diferencia de los compuestos antes mencionados, el incremento de $a$ con $x$ se debe en su totalidad a la mayor diferencia en los radios metálicos $\left(r_{m e t}\right)$ de los átomos de In y Fe $(1.66 \AA$ y $1.24 \AA$, respectivamente [190]). Este comportamiento es avalado con los valores experimentales publicados en [152]. Dichas dependencias son descritas con las siguientes funciones lineales:

$$
\begin{aligned}
& a^{\text {cal }}(x)=3.7900_{1}+0.1019_{1} x \\
& a^{\exp }(x)=3.7998_{2}+0.0962_{2} x
\end{aligned}
$$


Además, del hecho que algunos átomos FeI son reemplazados por átomos de $M$, es posible distinguir diferentes tipos de átomos FeI, FeII y $M$ de acuerdo al entorno que poseen, es decir el tipo de átomos existentes como primeros $(n n) \mathrm{y} / \mathrm{o}$ segundos $(n n n)$ vecinos. En las Tablas 5.5 y 5.6 se reportan los diferentes tipos de FeI, FeII y $M$ de acuerdo a su entorno, para los diferentes valores de $x$. Para el caso del Al e In (Tabla 5.5) los nn y nnn son semejantes porque tienen las mismas configuraciones de equilibrio (Tabla 5.3), mientras que para el Ga, los nn y nnn se muestran en Tabla 5.6. 
Tabla 5.5: Diferentes tipos de átomos FeI y FeII de acuerdo a sus primeros $(n n)$ y segundos vecinos $(n n n)$ correspondiente a los distintos valores de $(x)$ de los compuestos $M_{x} \mathrm{Fe}_{4-x} \mathrm{~N}(\mathrm{M}=\mathrm{Al}$ o In). El número en paréntesis representa la cantidad de dicho tipo de átomo.

\begin{tabular}{|c|c|c|c|}
\hline$x$ & & $n n$ & $n n n$ \\
\hline \multirow[t]{2}{*}{0.000} & FeIa(8) & 12FeIIa & $8 \mathrm{~N}$ \\
\hline & FeIIa(24) & $2 \mathrm{~N}$ & $4 \mathrm{Fe} I a, 8 \mathrm{Fe} I I a$ \\
\hline \multirow[t]{5}{*}{0.125} & FeIa(3) & $8 \mathrm{Fe} I I a, 4 \mathrm{Fe} I I b$ & $8 \mathrm{~N}$ \\
\hline & $\mathrm{Fe} I b(3)$ & 4FeIIa, 8FeIIb & $8 \mathrm{~N}$ \\
\hline & $\mathrm{Fe} I c(1)$ & $12 \mathrm{Fe} I I b$ & $8 \mathrm{~N}$ \\
\hline & FeIIa(12) & $2 \mathrm{~N}$ & $1 \mathrm{MI} a, 2 \mathrm{Fe} I a, 1 \mathrm{Fe} I b, 4 \mathrm{Fe} I I a, 4 \mathrm{Fe} I I b$ \\
\hline & $\mathrm{Fe} I I b(12)$ & $2 \mathrm{~N}$ & 1Fe $I a, 2 \mathrm{Fe} I b, 1 \mathrm{Fe} I c, 4 \mathrm{Fe} I I a, 4 \mathrm{Fe} I I b$ \\
\hline \multirow[t]{5}{*}{0.250} & FeIa(4) & $4 \mathrm{Fe} I I a, 4 \mathrm{Fe} I I b, 4 \mathrm{Fe} I I c$ & $8 \mathrm{~N}$ \\
\hline & $\mathrm{Fe} I b(2)$ & $4 \mathrm{Fe} I I a, 8 \mathrm{Fe} I I c$ & $8 \mathrm{~N}$ \\
\hline & FeIIa(8) & $2 \mathrm{~N}$ & $1 \mathrm{MI} a, 2 \mathrm{Fe} I a, 1 \mathrm{Fe} I b, 4 \mathrm{Fe} I I b, 4 \mathrm{Fe} I I c$ \\
\hline & $\mathrm{Fe} I I b(8)$ & $2 \mathrm{~N}$ & 2MIa, 2FeIa, 4FeIIa, 2FeIIb, 2FeIIc \\
\hline & $\mathrm{Fe} I \mathrm{Ic}(8)$ & $2 \mathrm{~N}$ & 2FeIa, 2FeIb, 4FeIIa, 2FeIIb, 2FeIIc \\
\hline \multirow[t]{8}{*}{0.375} & FeIa(1) & $4 \mathrm{Fe} I I b, 8 \mathrm{Fe} I I c$ & $8 \mathrm{~N}$ \\
\hline & $\mathrm{Fe} I b(1)$ & $8 \mathrm{Fe} I I a, 4 \mathrm{Fe} I I d$ & $8 \mathrm{~N}$ \\
\hline & $\mathrm{Fe} I c(2)$ & 4FeIIa, 4FeIIc, 4FeIId & $8 \mathrm{~N}$ \\
\hline & FeId(1) & 8FeIIc, 8FeIId & $8 \mathrm{~N}$ \\
\hline & FeIIa(8) & $2 \mathrm{~N}$ & $1 \mathrm{MI} a, 1 \mathrm{MI} b, 1 \mathrm{Fe} I b, 1 \mathrm{Fe} I c, 2 \mathrm{Fe} I I a, 2 \mathrm{Fe} I I b, 2 \mathrm{Fe} I I c, 2 \mathrm{Fe} I I d$ \\
\hline & $\mathrm{Fe} I I b(4)$ & $2 \mathrm{~N}$ & $1 \mathrm{MI} a, 2 \mathrm{MI} b, 1 \mathrm{Fe} I a, 4 \mathrm{Fe} I I a, 4 \mathrm{Fe} I I c$ \\
\hline & FeIIc(8) & $2 \mathrm{~N}$ & $1 \mathrm{MI} b, 1 \mathrm{Fe} I a, 1 \mathrm{Fe} I c, 1 \mathrm{Fe} I d, 2 \mathrm{Fe} I I a, 2 \mathrm{Fe} I I b, 2 \mathrm{Fe} I I c, 2 \mathrm{Fe} I I d$ \\
\hline & $\mathrm{Fe} I I d(4)$ & $2 \mathrm{~N}$ & 1FeIb, 2FeIc, 1FeId, 4FeIIa, 4FeIIc \\
\hline \multirow[t]{4}{*}{0.500} & $\operatorname{Fe} I a(4)$ & $8 \mathrm{Fe} I I a, 4 \mathrm{Fe} I I c$ & $8 \mathrm{~N}$ \\
\hline & FeIIa(16) & $2 \mathrm{~N}$ & 2MIa, 2FeIa, 4FeIIa, 2FeIIb, 2FeIIc \\
\hline & FeIIb(4) & $2 \mathrm{~N}$ & $4 \mathrm{MI} a, 8 \mathrm{Fe} I I a$ \\
\hline & FeIIc(4) & $2 \mathrm{~N}$ & $4 \mathrm{Fe} I a, 8 \mathrm{Fe} I I a$ \\
\hline \multirow[t]{6}{*}{0.625} & FeI $a(2)$ & 4FeIIa, 4FeIIc, 4FeIId & $8 \mathrm{~N}$ \\
\hline & $\mathrm{Fe} I b(1)$ & $8 \mathrm{Fe} I I c, 4 \mathrm{Fe} I I d$ & $8 \mathrm{~N}$ \\
\hline & FeIIa(8) & $2 \mathrm{~N}$ & $1 \mathrm{MI} a, 1 \mathrm{MI} l, 1 \mathrm{MI} d, 1 \mathrm{Fe} I a, 2 \mathrm{Fe} I I a, 2 \mathrm{Fe} I I b, 2 \mathrm{Fe} I I c, 2 \mathrm{Fe} I I d$ \\
\hline & $\mathrm{Fe} I I b(4)$ & $2 \mathrm{~N}$ & $1 \mathrm{MIa}, 2 \mathrm{MIb}, 1 \mathrm{M} I c, 4 \mathrm{Fe} I I a, 4 \mathrm{Fe} I I c$ \\
\hline & FeIIc(8) & $2 \mathrm{~N}$ & $1 \mathrm{MI} b, 1 \mathrm{M} I c, 1 \mathrm{Fe} I a, 1 \mathrm{Fe} I b, 2 \mathrm{Fe} I I a, 2 \mathrm{Fe} I I b, 2 \mathrm{Fe} I I c, 2 \mathrm{Fe} I I d$ \\
\hline & FeIId(4) & $2 \mathrm{~N}$ & $1 \mathrm{M} I d, 2 \mathrm{Fe} I a, 1 \mathrm{Fe} I b, 4 \mathrm{Fe} I I a, 4 \mathrm{Fe} I I c$ \\
\hline \multirow[t]{4}{*}{0.750} & FeI $I(2)$ & 4FeIIa, 8FeIIc & $8 \mathrm{~N}$ \\
\hline & FelIa(8) & $2 \mathrm{~N}$ & $1 \mathrm{MI} a, 2 \mathrm{MIb}, 1 \mathrm{Fe} I a, 4 \mathrm{Fe} I I b, 4 \mathrm{Fe} I I c$ \\
\hline & $\mathrm{Fe} I I b(8)$ & $2 \mathrm{~N}$ & $1 \mathrm{MI} a, 2 \mathrm{MI} b, 4 \mathrm{Fe} I I a, 2 \mathrm{Fe} I I b, 2 \mathrm{Fe} I I c$ \\
\hline & FeIIc(8) & $2 \mathrm{~N}$ & $2 \mathrm{MI} b, 2 \mathrm{Fe} I a, 4 \mathrm{Fe} I I a, 2 \mathrm{Fe} I I b, 2 \mathrm{Fe} I I c$ \\
\hline \multirow[t]{3}{*}{0.875} & FeI $a(1)$ & $12 \mathrm{Fe} I I a$ & $8 \mathrm{~N}$ \\
\hline & FeIIa(12) & $2 \mathrm{~N}$ & 2MIa, 1MIb, 1FeIa, 4FeIIa, 4FeIIb \\
\hline & $\mathrm{Fe} I I b(12)$ & $2 \mathrm{~N}$ & $1 \mathrm{MI} a, 2 \mathrm{M} I b, 1 \mathrm{M} I c, 4 \mathrm{Fe} I I a, 4 \mathrm{Fe} I I b$ \\
\hline 1.000 & $\mathrm{Fe} I a(24)$ & $2 \mathrm{~N}$ & $4 \mathrm{MIa}$ \\
\hline
\end{tabular}


Tabla 5.6: Diferentes tipos de átomos Fel y FeII de acuerdo a sus primeros $(n n)$ y segundos vecinos $(n n n)$ correspondiente a los distintos valores de $(x)$ de los compuestos $M_{x} \mathrm{Fe}_{4-x} \mathrm{~N}(M=\mathrm{Ga})$. El número en paréntesis representa la cantidad de dicho tipo de átomo.

\begin{tabular}{|c|c|c|c|}
\hline$x$ & & $n n$ & nnn \\
\hline \multirow[t]{2}{*}{0.000} & $\operatorname{Fe} I a(8)$ & 12FeIIa & $8 \mathrm{~N}$ \\
\hline & FeIIa(24) & $2 \mathrm{~N}$ & $4 \mathrm{Fe} I a, 8 \mathrm{Fe} I I a$ \\
\hline \multirow[t]{5}{*}{0.125} & $\operatorname{Fe} I a(3)$ & $8 \mathrm{Fe} I I a, 4 \mathrm{Fe} I I b$ & $8 \mathrm{~N}$ \\
\hline & $\mathrm{Fe} I b(3)$ & $4 \mathrm{Fe} I I a, 8 \mathrm{Fe} I I b$ & $8 \mathrm{~N}$ \\
\hline & $\mathrm{Fe} I c(1)$ & $12 \mathrm{Fe} I I b$ & $8 \mathrm{~N}$ \\
\hline & FeIIa(12) & $2 \mathrm{~N}$ & 1MIa, 2FeIa, 1FeIc, 4FeIIa, 4FeIIb \\
\hline & $\mathrm{Fe} I I b(12)$ & $2 \mathrm{~N}$ & 1Fe $I a, 2 \mathrm{Fe} I b, 1 \mathrm{Fe} I c, 4 \mathrm{Fe} I I a, 4 \mathrm{Fe} I I b$ \\
\hline \multirow[t]{2}{*}{0.250} & FeIa(6) & $12 \mathrm{Fe} I I a$ & $8 \mathrm{~N}$ \\
\hline & FeIIa(24) & $2 \mathrm{~N}$ & $1 \mathrm{MI} a, 3 \mathrm{Fe} I a, 8 \mathrm{Fe} I I a$ \\
\hline \multirow[t]{5}{*}{0.375} & $\operatorname{Fe} I a(3)$ & $8 \mathrm{Fe} I I b, 4 \mathrm{Fe} I I b$ & $8 \mathrm{~N}$ \\
\hline & $\mathrm{Fe} I b(1)$ & $12 \mathrm{Fe} I I a$ & $8 \mathrm{~N}$ \\
\hline & $\operatorname{Fe} I c(1)$ & $12 \mathrm{Fe} I \mathrm{Ilb}$ & $8 \mathrm{~N}$ \\
\hline & FeIIa(12) & $2 \mathrm{~N}$ & 1MIa, 2Fe $I a, 1 \mathrm{Fe} I b, 4 \mathrm{Fe} I I a, 4 \mathrm{Fe} I I b$ \\
\hline & $\mathrm{Fe} I I b(12)$ & $2 \mathrm{~N}$ & 2MIa, 1FeIa, 1FeIc, 4FeIIa, 4FeIIb \\
\hline \multirow[t]{4}{*}{0.500} & $\mathrm{Fe} I a(3)$ & $4 \mathrm{Fe} I I a, 8 \mathrm{Fe} I I b$ & $8 \mathrm{~N}$ \\
\hline & $\mathrm{Fe} I b(1)$ & $12 \mathrm{Fe} I \mathrm{Il}$ & $8 \mathrm{~N}$ \\
\hline & $\mathrm{Fe} I I b(12)$ & $2 \mathrm{~N}$ & $2 \mathrm{M} I a, 1 \mathrm{M} I b, 1 \mathrm{Fe} I a, 4 \mathrm{Fe} I I a, 4 \mathrm{Fe} I I b$ \\
\hline & $\mathrm{Fe} I I c(12)$ & $2 \mathrm{~N}$ & 1MIa, 2FeIa, 1FeIb, 4FeIIa, 4FeIIb \\
\hline \multirow[t]{6}{*}{0.625} & FeIa(2) & 4FeIIa, 4FeIIc, 4FeIId & $8 \mathrm{~N}$ \\
\hline & $\mathrm{Fe} I b(1)$ & $8 \mathrm{Fe} I I c, 4 \mathrm{Fe} I I d$ & $8 \mathrm{~N}$ \\
\hline & FeIIa(8) & $2 \mathrm{~N}$ & $1 \mathrm{MI} a, 1 \mathrm{MI} b, 1 \mathrm{MId}, 1 \mathrm{Fe} I a, 2 \mathrm{Fe} I I a, 2 \mathrm{Fe} I I b, 2 \mathrm{Fe} I I c, 2 \mathrm{FeIId}$ \\
\hline & $\mathrm{Fe} I I b(4)$ & $2 \mathrm{~N}$ & 1MIa, 2MIb, 1MIc, 4FeIIa, 4FeIIc \\
\hline & FeIIc(8) & $2 \mathrm{~N}$ & $1 \mathrm{MI}$, $1 \mathrm{M} I c$, 1FeIa, 1FeIb, 2FeIIa, 2FeIIb, 2FeIIc, 2FeIId \\
\hline & FeIId(4) & $2 \mathrm{~N}$ & 1MId, 2FeIa, 1FeIb, 4FeIIa, 4FeIIc \\
\hline \multirow[t]{4}{*}{0.750} & $\mathrm{Fe} I a(2)$ & $4 \mathrm{Fe} I I a, 8 \mathrm{Fe} I I c$ & $8 \mathrm{~N}$ \\
\hline & $\mathrm{Fe} I I a(8)$ & $2 \mathrm{~N}$ & 1MIa, 2MIb, 1FeIa, 4FeIIb, 4FeIIc \\
\hline & $\mathrm{Fe} I \mathrm{Ib}(8)$ & $2 \mathrm{~N}$ & $1 \mathrm{MI} a, 2 \mathrm{MI} b, 4 \mathrm{Fe} I I a, 2 \mathrm{Fe} I I b, 2 \mathrm{Fe} I I c$ \\
\hline & FeIIc(8) & $2 \mathrm{~N}$ & $2 \mathrm{MIb}, 2 \mathrm{Fe} I a, 4 \mathrm{Fe} I I a, 2 \mathrm{Fe} I I b, 2 \mathrm{Fe} I I c$ \\
\hline \multirow[t]{3}{*}{0.875} & $\operatorname{Fe} I a(1)$ & $12 \mathrm{Fe} I I a$ & $8 \mathrm{~N}$ \\
\hline & FeIIa(12) & $2 \mathrm{~N}$ & $2 \mathrm{MI} a, 1 \mathrm{MI} I, 1 \mathrm{Fe} I a, 4 \mathrm{Fe} I I a, 4 \mathrm{Fe} I I b$ \\
\hline & $\mathrm{Fe} I I b(12)$ & $2 \mathrm{~N}$ & 1MIa, 2MIb, 1MIc, 4FeIIa, 4FeIIb \\
\hline 1.000 & $\mathrm{Fe} I a(24)$ & $2 \mathrm{~N}$ & $4 \mathrm{MIa}$ \\
\hline
\end{tabular}




\subsubsection{Estructura Electrónica, Propiedades Magnéticas e Hi- perfinas}

La modificación del entorno ( $n n$ y/o $n n n)$ de los átomos de Fe (FeI o FeII) produce cambios en la estructura electrónica de los mismos, viéndose esto reflejado en las propiedades magnéticas e hiperfinas de dichos átomos. En las Figuras 5.5 y 5.6 se muestran las densidades de estados (DOS) para los compuestos $M_{0.50} \mathrm{Fe}_{3.50} \mathrm{~N}$ $(x=0.50)$ y $M_{1.00} \mathrm{Fe}_{3.00} \mathrm{~N}(x=1.00)$, respectivamente, donde se puede observar que los estados electrónicos de los FeI se concentran entre el nivel de Fermi $\left(E_{f}\right)$ y $-5.0 \mathrm{eV}$, mientras que los estrados electrónicos de los FeII se expanden hasta aproximadamente $-9.0 \mathrm{eV}$, mostrando una fuerte hibridización entre los orbitales $d$-FeI y $d$-FeII característico del $\gamma^{\prime}-\mathrm{Fe}_{4} \mathrm{~N}$ [130]. Para energías menores de -5.0eV los estados electrónicos $d$-FeII se encuentran entrelazados con los estados electrónicos $s-M$, $p$-N [156] indicando una interacción entre los átomos FeII y los átomos $M$ ubicados como segundo vecinos, además de una menor hibridización entre los orbitales $d$-FeII y $p-M$ localizados entre los $-2.0 \mathrm{eV}$ y $-5.0 \mathrm{eV}$, la cual es más notable conforme aumenta $x$ (Figura 5.6) debido a la ocupación de estados electrónicos $p$ - $M$ en ese rango de energía [156]. Se puede notar también la ausencia de alguna hibridización entre orbitales de los $\mathrm{Fe} I$ con el $M$ lo cual indica que entre estos átomos no existe interacción alguna. 


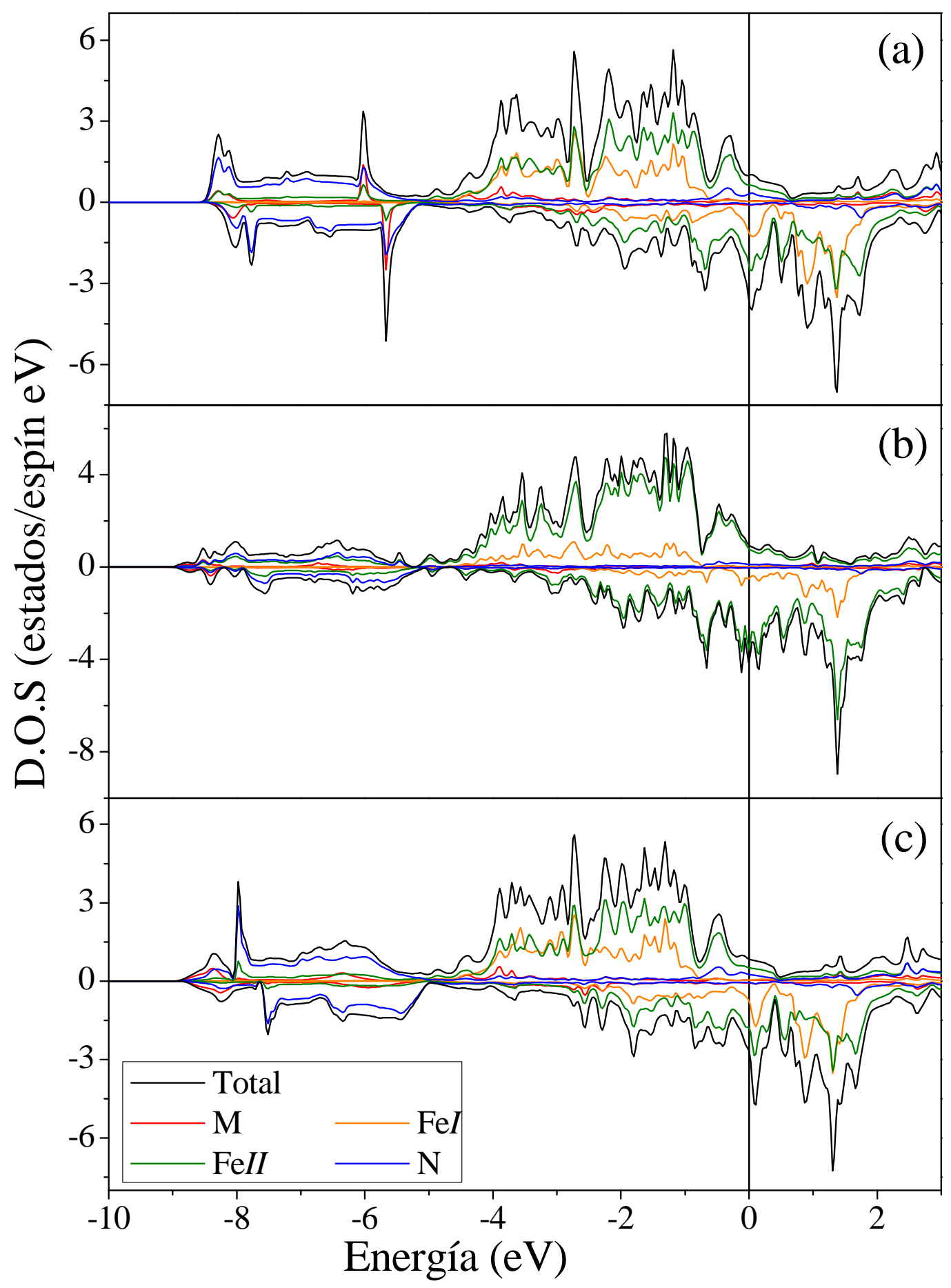

Figura 5.5: Densidad de estados electrónicos (DOS) de los compuestos a) $\mathrm{Al}_{0.500} \mathrm{Fe}_{3.500} \mathrm{~N}$, b) $\mathrm{Ga}_{0.500} \mathrm{Fe}_{3.500} \mathrm{~N}$ y c) $\operatorname{In}_{0.500} \mathrm{Fe}_{3.500} \mathrm{~N}$, totales y por átomo. 


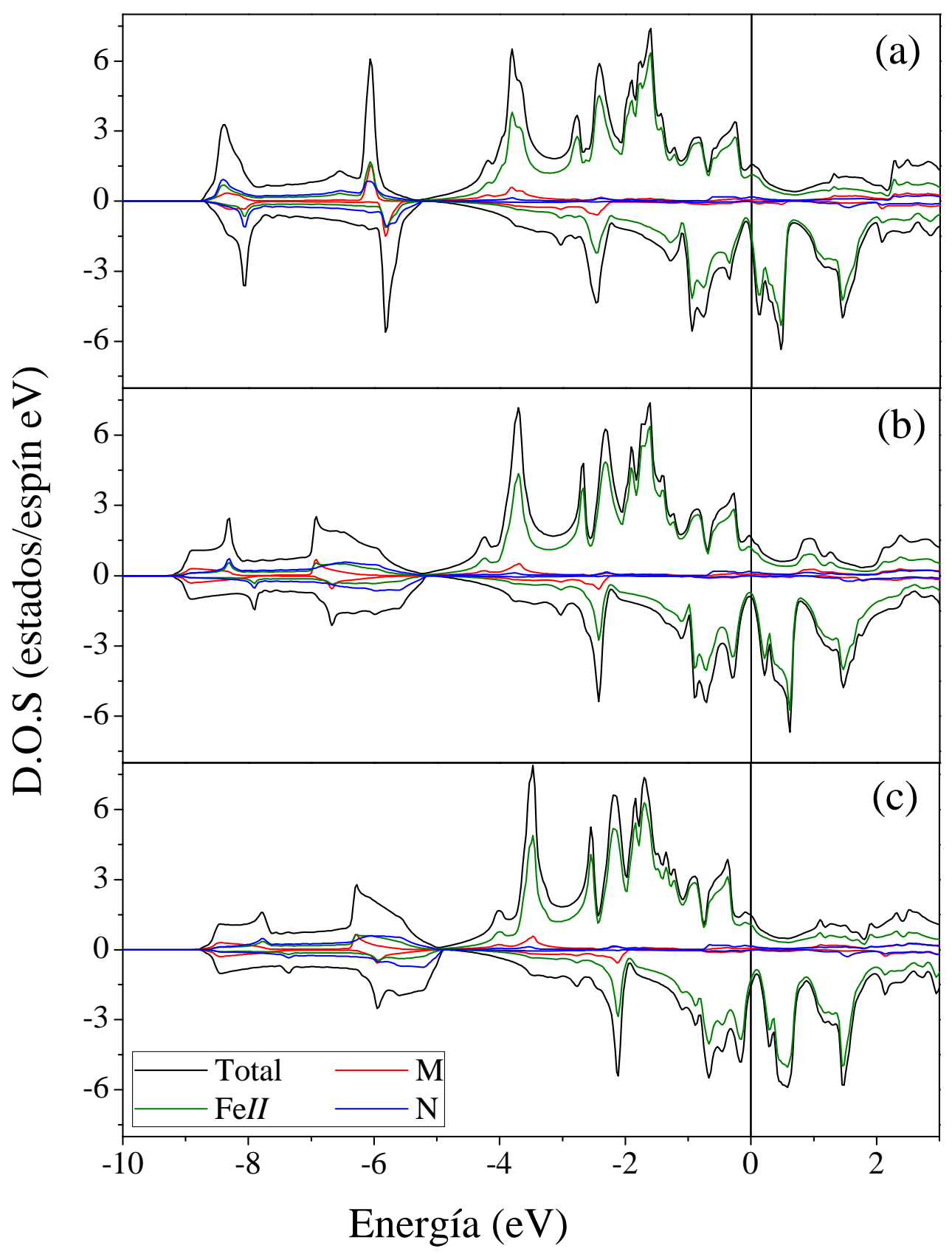

Figura 5.6: Densidad de estados electrónicos (DOS) de los compuestos a) $\mathrm{Al}_{1.000} \mathrm{Fe}_{3.000} \mathrm{~N}$, b) $\mathrm{Ga}_{1.000} \mathrm{Fe}_{3.000} \mathrm{~N}$ y c) $\operatorname{In}_{1.000} \mathrm{Fe}_{3.000} \mathrm{~N}$, totales y por átomo. 
El entrelazamiento de las bandas de los átomos de los compuestos $M_{x} \mathrm{Fe}_{4-x} \mathrm{~N}$, produce una modificación en la distribución de las cargas totales de los átomos Fe. En la Figura 5.7 se muestran las cargas de los orbitales $3 d$-FeI y $3 d$-FeII en función de $x$, donde se puede apreciar que las cargas $3 d$-up y $3 d$-down de los FeI no varían, mientras que para los FeII, se observa una disminución de la carga $3 d$-up y un incremento de la carga $3 d$-down conforme aumenta $x$ pero manteniéndose invariante la carga total del orbital $3 d$-FeII. Esto indica una redistribución de la carga del orbital $3 d$-FeII debido a la transferencia de estados electrónico $3 d$-up hacia los 3d-down o a un desplazamiento de estados $3 d$-up a energías mayores del $E_{f}$ simultáneamente con un desplazamiento de estados 3d-down a energías menores del $E_{f}$, los cuales dependen de $x$. Este comportamiento puede estar relacionado con la interacción magnética existente entre los átomos $M$ y los átomos de Fe.

La dependencia con $x$ de la carga en el orbital $3 d$ de los átomos de Fe está relacionada directamente con el momento magnético de dichos átomos. En la Figura 5.8 se muestran los momentos magnéticos de los átomos $M$ y de los distintos átomos FeI y FeII en función de $x$ para los compuestos $M_{x} \mathrm{Fe}_{4-x} \mathrm{~N}$, donde se puede apreciar que el momento magnético de los átomos FeI no varían con $x, 2.9 \mu_{B}$ semejante al FeI del $\gamma^{\prime}-\mathrm{Fe}_{4} \mathrm{~N}[52,82,129,130]$, esto se debe a que la carga en los estados 3d-up y 3d-down de los FeI tampoco varia con $x$ (Figura 5.7). Para los átomos FeII, se pude apreciar un decrecimiento gradual de sus momentos magnéticos conforme aumenta $x$. Este comportamiento está relacionado directamente con la variación de las cargas 3d-up y $3 d$-down descritas anteriormente. 


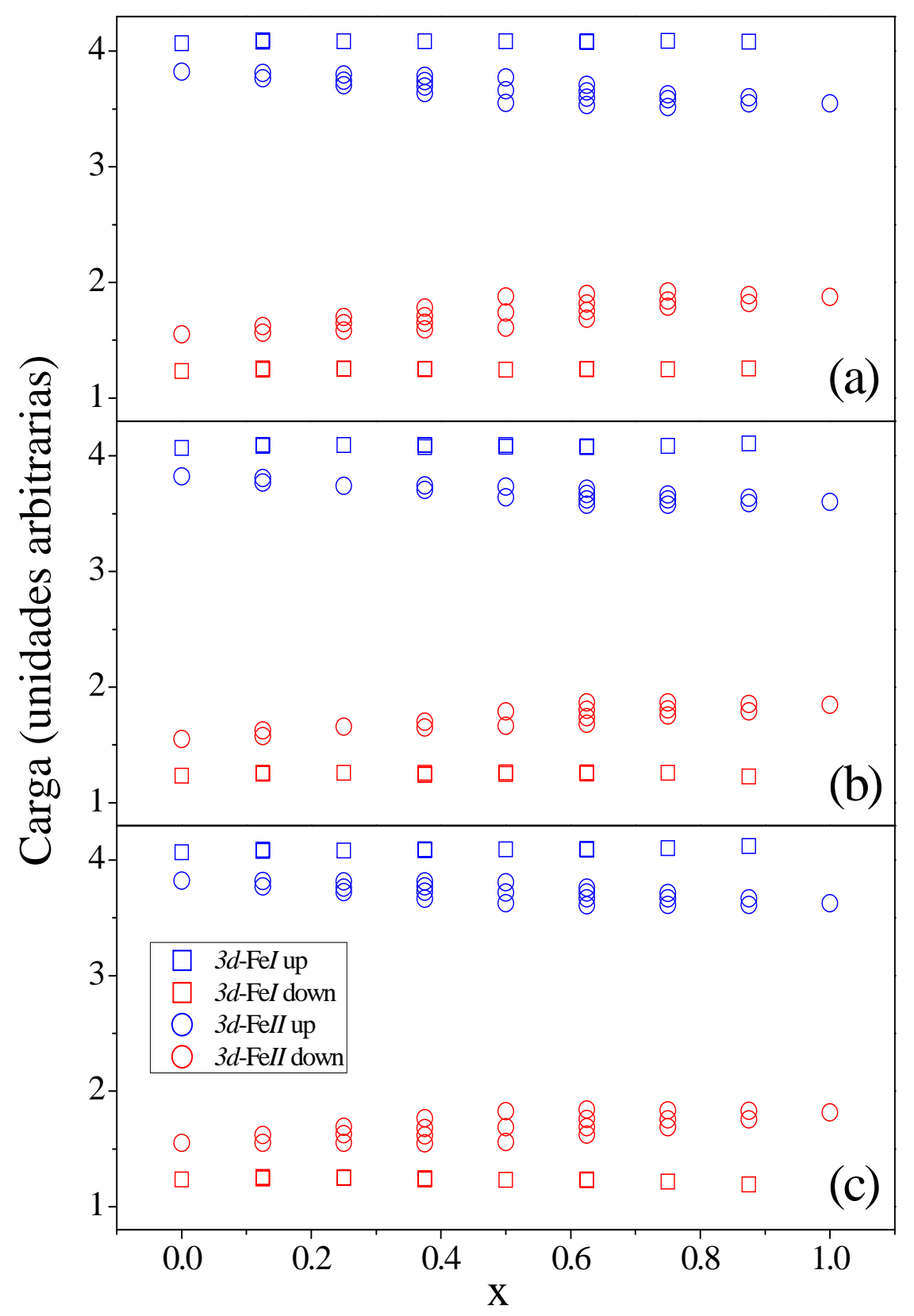

Figura 5.7: Carga del orbital 3d-up y $3 d$-down de los átomos FeI y FeII en función de $x$ para los compuestos a) $\left.\mathrm{Al}_{x} \mathrm{Fe}_{4-x} \mathrm{~N}, \mathrm{~b}\right) \mathrm{Ga}_{x} \mathrm{Fe}_{4-x} \mathrm{~N}$ y c) $\mathrm{In}_{x} \mathrm{Fe}_{4-x} \mathrm{~N}$. 
Mientras que los átomos $M(\mathrm{Al}, \mathrm{Ga}, \mathrm{In})$ muestran un pequeño momento magnético que no depende de $x$, pero si muestran una alineación antiparalela al momento magnético de los átomos de $\mathrm{Fe}$, cuyos valores son $-0.15 \mu_{B},-0.11 \mu_{B} \mathrm{y}-0.12 \mu_{B}$ respectivamente. La no dependencia con $x$ del momento magnético de los átomos FeI, podría estar relacionada con que el entorno de dichos átomos no depende de $x$, es decir, los átomos FeI siempre tendrán 12 átomos de Fe a $\frac{\sqrt{2}}{2} a$ como $n n$ y a 8 átomos de $\mathrm{N}$ a $\frac{\sqrt{3}}{2} a$ como $n n n$, además de la ausencia de alguna hibridización entre los orbitales de los FeI y del átomos $M$ (Figura 5.5 y 5.6). Esto es distinto para el caso de los átomos FeII, los que siempre tendrán 2 átomos de $\mathrm{N}$ como $n n$ a $\frac{a}{2}$, mientras que el tipo de átomos $n n n$ dependen de $x$, pudiendo variar la cantidad de $n n n$ desde 0 hasta 4 átomos $M$ a $\frac{\sqrt{2}}{2} a$ (Tablas 5.5 y 5.6), cabe mencionar que a pesar que los átomos $M$ se encuentran como nnn de los átomos FeII, la distancia que separa a dichos átomos es semejante a la que existe entre el FeI y sus $n n$.

Al graficar el momento magnético de los átomos de FeII en función del número átomos $M$ como nnn (Figura 5.9), se puede apreciar un decrecimiento lineal de las mismas, cuyas pendientes son $-0.14 \mu_{B},-0.13 \mu_{B} \mathrm{y}-0.12 \mu_{B}$ por átomo $n n n$ de $\mathrm{Al}$, $\mathrm{Ga}$, In, respectivamente. Esto indica que el decrecimiento del momento magnético de los átomos FeII dependen fuertemente de la cantidad de átomos $M$ ubicados como $n n n$, los cuales interactúan antiferromagnéticamente, ocasionando así una redistribución de la carga del orbital $3 d$ de los átomos FeII. 


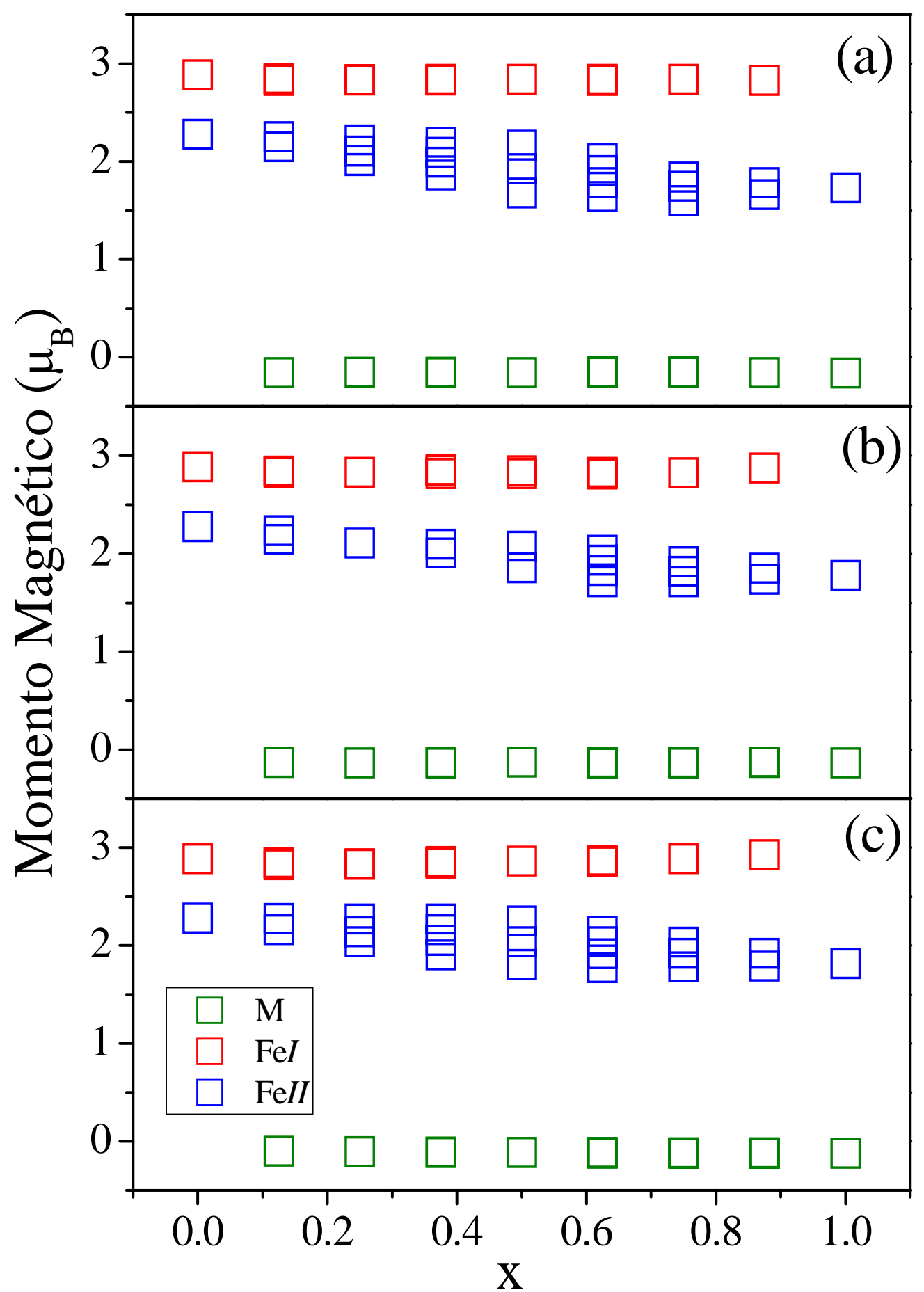

Figura 5.8: Momentos magnéticos de los átomos $M$, FeI y FeII en función de $x$, para los compuestos a) $\left.\mathrm{Al}_{x} \mathrm{Fe}_{4-x} \mathrm{~N}, \mathrm{~b}\right) \mathrm{Ga}_{x} \mathrm{Fe}_{4-x} \mathrm{~N}$ y c) $\mathrm{In}_{x} \mathrm{Fe}_{4-x} \mathrm{~N}$. 


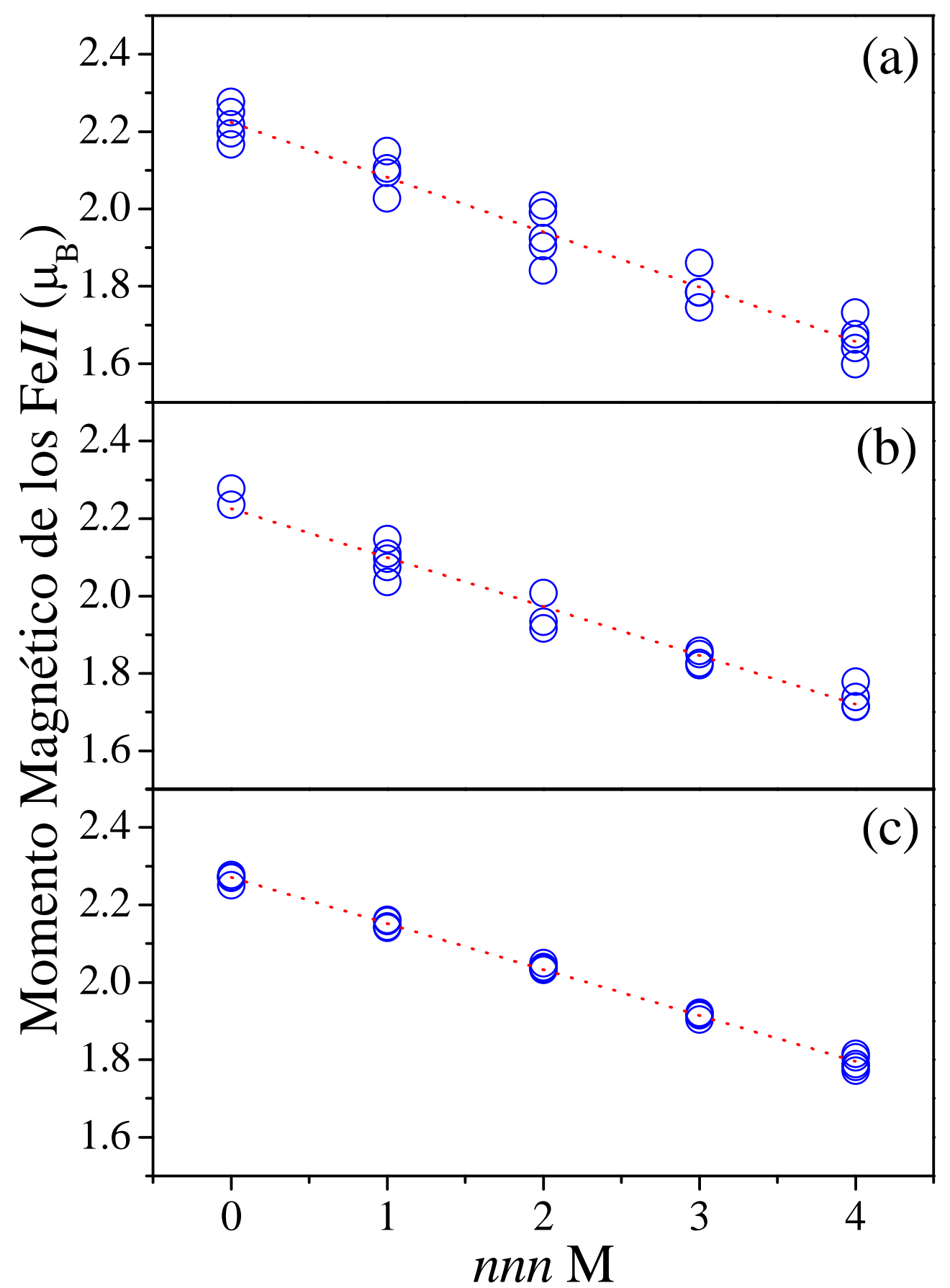

Figura 5.9: Dependencia del momento magnético de los FeII con la cantidad de átomos $M$ como nnn para los compuestos a) $\left.\mathrm{Al}_{x} \mathrm{Fe}_{4-x} \mathrm{~N}, \mathrm{~b}\right) \mathrm{Ga}_{x} \mathrm{Fe}_{4-x} \mathrm{~N} \mathrm{y} \mathrm{c)} \operatorname{In}_{x} \mathrm{Fe}_{4-x} \mathrm{~N}$. 
Luego, la disminución del momento magnético de los átomos FeII con $x$ (o con el número de $M n n n$ ) ocasiona un decrecimiento en el momento magnético total $\left(M_{f u}\right)$ de los compuestos $M_{x} \mathrm{Fe}_{4-x} \mathrm{~N}$, cuyos valores se reportan en la Tabla 5.7. En la Figura 5.10 se muestran los valores de $M_{f u}$ respecto a $x$, observándose un decrecimiento lineal de $M_{f u}$ cuyas razones de cambio son de $-4.8 \mu_{B},-4.6 \mu_{B}$ y $-4.3 \mu_{B}$ para los compuestos $\mathrm{Al}_{x} \mathrm{Fe}_{4-x} \mathrm{~N}, \mathrm{Ga}_{x} \mathrm{Fe}_{4-x} \mathrm{~N}$ y $\operatorname{In}_{x} \mathrm{Fe}_{4-x} \mathrm{~N}$, respectivamente, donde el $M_{f u}$ para los compuestos $\mathrm{AlFe}_{3} \mathrm{~N}, \mathrm{GaFe}_{3} \mathrm{~N}, \mathrm{InFe}_{3} \mathrm{~N}(x=1)$ es casi la mitad que del $\gamma^{\prime}-\mathrm{Fe}_{4} \mathrm{~N}(x=0)$. Mientras que para los compuestos $\mathrm{Ga}_{x} \mathrm{Fe}_{4-x} \mathrm{~N}$ y $\operatorname{In}_{x} \mathrm{Fe}_{4-x} \mathrm{~N}$, las razones de cambios del $M_{f u}$ experimentales son de $-9.7 \mu_{B}$ [155] y $-7.81 \mu_{B}$ [152], respectivamente, conllevando a valores de $M_{f u}$ cercanos a $1 \mu_{B}$ $(x=1)$. Los autores de dichos trabajos experimentales proponen al $\mathrm{GaFe}_{3} \mathrm{~N}$ y al $\mathrm{InFe}_{3} \mathrm{~N}$ como materiales antiferromagnéticos $[152,155]$ a pesar de que sus medidas de magnetización no han sido saturadas. Mientras que estudios teóricos proponen a estos compuestos como posibles ferromagnetos [156].

Tabla 5.7: Valores del $M_{f u}$ en función de $x$ para los compuestos $M_{x} \mathrm{Fe}_{4-x} \mathrm{~N}$.

\begin{tabular}{ccccccccc}
\hline & \multicolumn{7}{c}{$M_{f u}\left(\mu_{B} / f u\right)$} \\
$x$ & 0.125 & 0.250 & 0.375 & 0.500 & 0.625 & 0.750 & 0.875 & 1.00 \\
\hline $\mathrm{Al}_{x} \mathrm{Fe}_{4-x} \mathrm{~N}$ & 9.10 & 8.45 & 7.85 & 7.13 & 6.50 & 5.78 & 5.39 & 5.14 \\
$\mathrm{Ga}_{x} \mathrm{Fe}_{4-x} \mathrm{~N}$ & 9.07 & 8.38 & 7.91 & 7.28 & 6.63 & 6.08 & 5.66 & 5.30 \\
$\mathrm{In}_{x} \mathrm{Fe}_{4-x} \mathrm{~N}$ & 9.11 & 8.58 & 8.06 & 7.51 & 6.93 & 6.38 & 5.84 & 5.48 \\
\hline
\end{tabular}




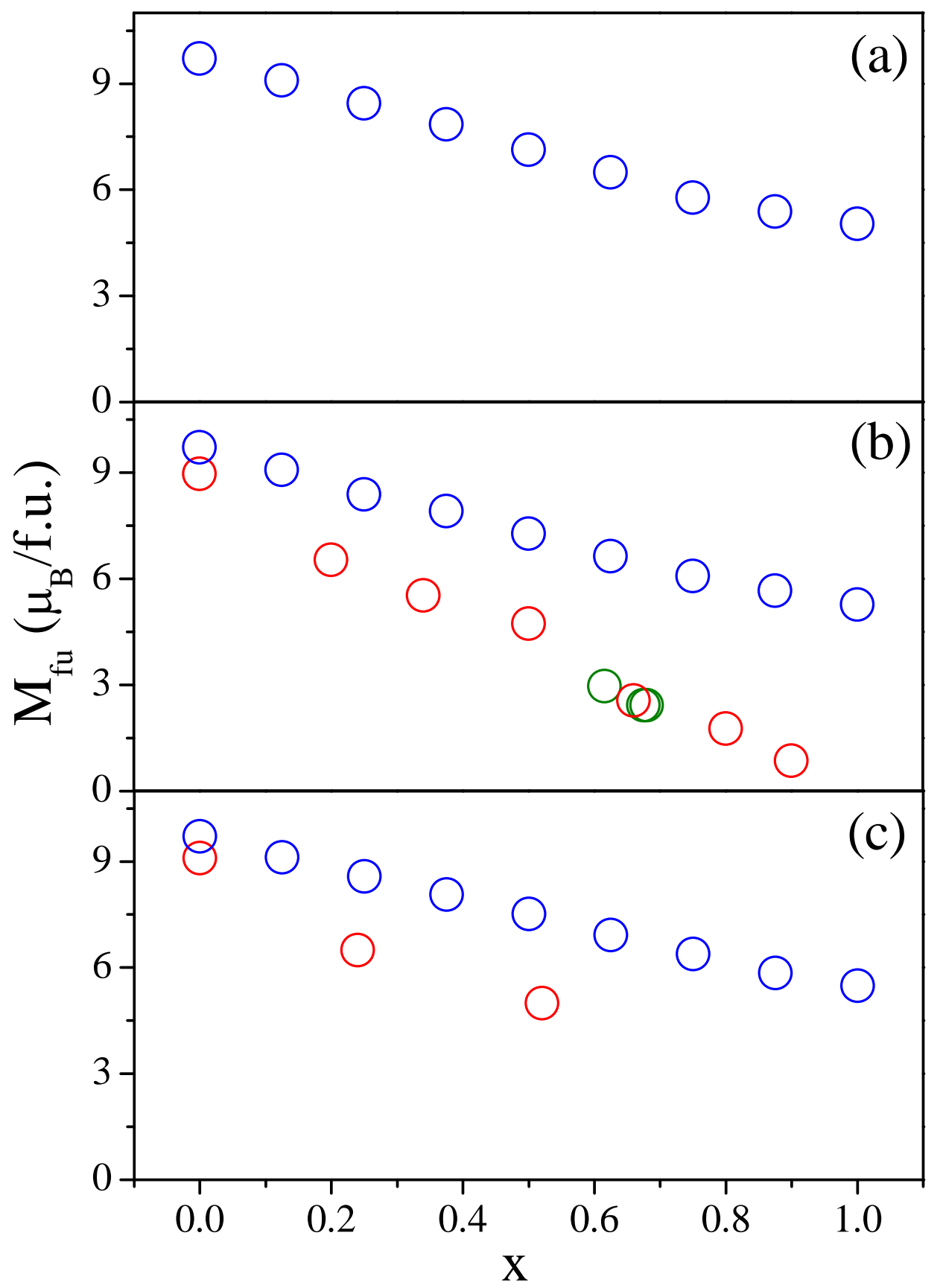

Figura 5.10: Variación del $M_{f u}$ respecto de $x$ para a) $\left.\mathrm{Al}_{x} \mathrm{Fe}_{4-x} \mathrm{~N}, \mathrm{~b}\right) \mathrm{Ga}_{x} \mathrm{Fe}_{4-x} \mathrm{~N}$, círculos verdes y rojos son datos experimentales de [151] y [155], respectivamente, c) $\operatorname{In}_{x} \mathrm{Fe}_{4-x} \mathrm{~N}$, círculos rojos son datos experimentales de [152]. 
Tabla 5.8: Valores del $B_{h f}(\mathrm{~T}), \delta(\mathrm{mm} / \mathrm{s})$ y $\varepsilon(\mathrm{mm} / \mathrm{s})$ de los diferentes átomos de Fe de los compuestos $M_{x} \mathrm{Fe}_{4-x} \mathrm{~N}$.

\begin{tabular}{|c|c|c|c|c|c|c|c|c|c|c|}
\hline \multirow[b]{2}{*}{$x$} & \multirow[b]{2}{*}{$\mathrm{Fe}$} & \multicolumn{3}{|c|}{$\mathrm{Al}_{x} \mathrm{Fe}_{4-x} \mathrm{~N}$} & \multicolumn{3}{|c|}{$\mathrm{Ga}_{x} \mathrm{Fe}_{4-x} \mathrm{~N}$} & \multicolumn{3}{|c|}{$\mathrm{In}_{x} \mathrm{Fe}_{4-x} \mathrm{~N}$} \\
\hline & & $B_{h f}$ & $\delta$ & $\varepsilon$ & $B_{h f}$ & $\delta$ & $\varepsilon$ & $B_{h f}$ & $\delta$ & $\varepsilon$ \\
\hline \multirow[t]{5}{*}{0.125} & $\mathrm{Fe} I$ & 40.1 & 0.33 & 0.05 & 40.1 & 0.29 & 0.08 & 40.5 & 0.33 & 0.07 \\
\hline & & 39.3 & 0.33 & 0.02 & 39.2 & 0.30 & 0.05 & 39.8 & 0.34 & 0.02 \\
\hline & & 39.3 & 0.33 & 0.00 & 38.5 & 0.30 & 0.00 & 40.0 & 0.34 & 0.00 \\
\hline & FeII & 22.3 & 0.38 & 0.55 & 23.0 & 0.36 & 0.61 & 22.4 & 0.39 & 0.54 \\
\hline & & 23.8 & 0.36 & 0.46 & 24.4 & 0.33 & 0.47 & 23.9 & 0.36 & 0.46 \\
\hline \multirow[t]{5}{*}{0.250} & $\mathrm{Fe} I$ & 39.1 & 0.32 & 0.13 & 38.9 & 0.29 & 0.01 & 40.2 & 0.34 & 0.17 \\
\hline & & 38.1 & 0.33 & 0.01 & & & & 39.2 & 0.34 & 0.00 \\
\hline & FeII & 22.4 & 0.38 & 0.48 & 22.7 & 0.36 & 0.59 & 22.8 & 0.40 & 0.45 \\
\hline & & 21.0 & 0.40 & 0.65 & & & & 21.2 & 0.43 & 0.66 \\
\hline & & 24.1 & 0.36 & 0.43 & & & & 24.5 & 0.38 & 0.44 \\
\hline \multirow[t]{8}{*}{0.375} & $\mathrm{Fe} I$ & 39.4 & 0.32 & 0.15 & 39.9 & 0.28 & 0.05 & 40.7 & 0.34 & 0.17 \\
\hline & & 38.2 & 0.32 & 0.19 & 37.7 & 0.29 & 0.00 & 39.6 & 0.34 & 0.30 \\
\hline & & 38.1 & 0.32 & 0.06 & 41.3 & 0.27 & 0.00 & 39.3 & 0.34 & 0.11 \\
\hline & & 37.3 & 0.32 & 0.03 & & & & 38.5 & 0.35 & 0.01 \\
\hline & FeII & 21.0 & 0.40 & 0.63 & 23.3 & 0.36 & 0.65 & 21.6 & 0.36 & 0.63 \\
\hline & & 19.1 & 0.42 & 0.74 & 21.7 & 0.39 & 0.70 & 19.3 & 0.25 & 0.77 \\
\hline & & 22.6 & 0.38 & 0.52 & & & & 23.2 & 0.42 & 0.49 \\
\hline & & 23.9 & 0.36 & 0.44 & & & & 24.6 & 0.39 & 0.43 \\
\hline \multirow[t]{5}{*}{0.500} & $\mathrm{Fe} I$ & 37.6 & 0.31 & 0.06 & 38.6 & 0.29 & 0.10 & 39.7 & 0.35 & 0.00 \\
\hline & & & & & 36.9 & 0.29 & 0.00 & & & \\
\hline & FeII & 20.8 & 0.39 & 0.59 & 19.8 & 0.44 & 0.88 & 21.7 & 0.45 & 0.63 \\
\hline & & 19.0 & 0.44 & 0.90 & 23.1 & 0.37 & 0.58 & 18.8 & 0.29 & 1.04 \\
\hline & & 25.6 & 0.37 & 0.40 & & & & 26.9 & 0.41 & 0.48 \\
\hline \multirow[t]{6}{*}{0.625} & $\mathrm{Fe} I$ & 37.1 & 0.31 & 0.11 & 38.6 & 0.28 & 0.09 & 39.7 & 0.36 & 0.20 \\
\hline & & 35.8 & 0.31 & 0.02 & 37.0 & 0.28 & 0.08 & 38.4 & 0.36 & 0.13 \\
\hline & FeII & 18.6 & 0.41 & 0.70 & 19.7 & 0.43 & 0.86 & 20.1 & 0.27 & 0.70 \\
\hline & & 16.8 & 0.43 & 0.86 & 17.9 & 0.46 & 1.07 & 17.7 & 0.30 & 0.93 \\
\hline & & 20.8 & 0.39 & 0.59 & 21.8 & 0.40 & 0.69 & 21.9 & 0.25 & 0.58 \\
\hline & & 22.1 & 0.38 & 0.56 & 22.7 & 0.37 & 0.58 & 23.3 & 0.44 & 0.53 \\
\hline \multirow[t]{4}{*}{0.750} & $\mathrm{FeI}$ & 36.0 & 0.31 & 0.18 & 37.6 & 0.29 & 0.09 & 39.0 & 0.35 & 0.28 \\
\hline & FeII & 18.5 & 0.42 & 0.73 & 19.8 & 0.44 & 0.84 & 20.0 & 0.28 & 0.72 \\
\hline & & 17.0 & 0.44 & 0.82 & 17.7 & 0.47 & 1.05 & 17.8 & 0.31 & 0.89 \\
\hline & & 20.4 & 0.41 & 0.59 & 21.3 & 0.41 & 0.69 & 21.7 & 0.26 & 0.63 \\
\hline \multirow[t]{3}{*}{0.875} & $\mathrm{Fe} I$ & 37.5 & 0.32 & 0.87 & 39.3 & 0.28 & 0.00 & 40.6 & 0.35 & 0.00 \\
\hline & FeII & 18.7 & 0.67 & 0.43 & 19.9 & 0.44 & 0.86 & 20.2 & 0.30 & 0.72 \\
\hline & & 16.9 & 0.87 & 0.44 & 17.9 & 0.47 & 1.04 & 18.3 & 0.32 & 0.83 \\
\hline 1.000 & $\mathrm{Fe} I I$ & 17.4 & 0.86 & 0.45 & 18.0 & 0.48 & 1.03 & 18.3 & 0.33 & 0.81 \\
\hline
\end{tabular}




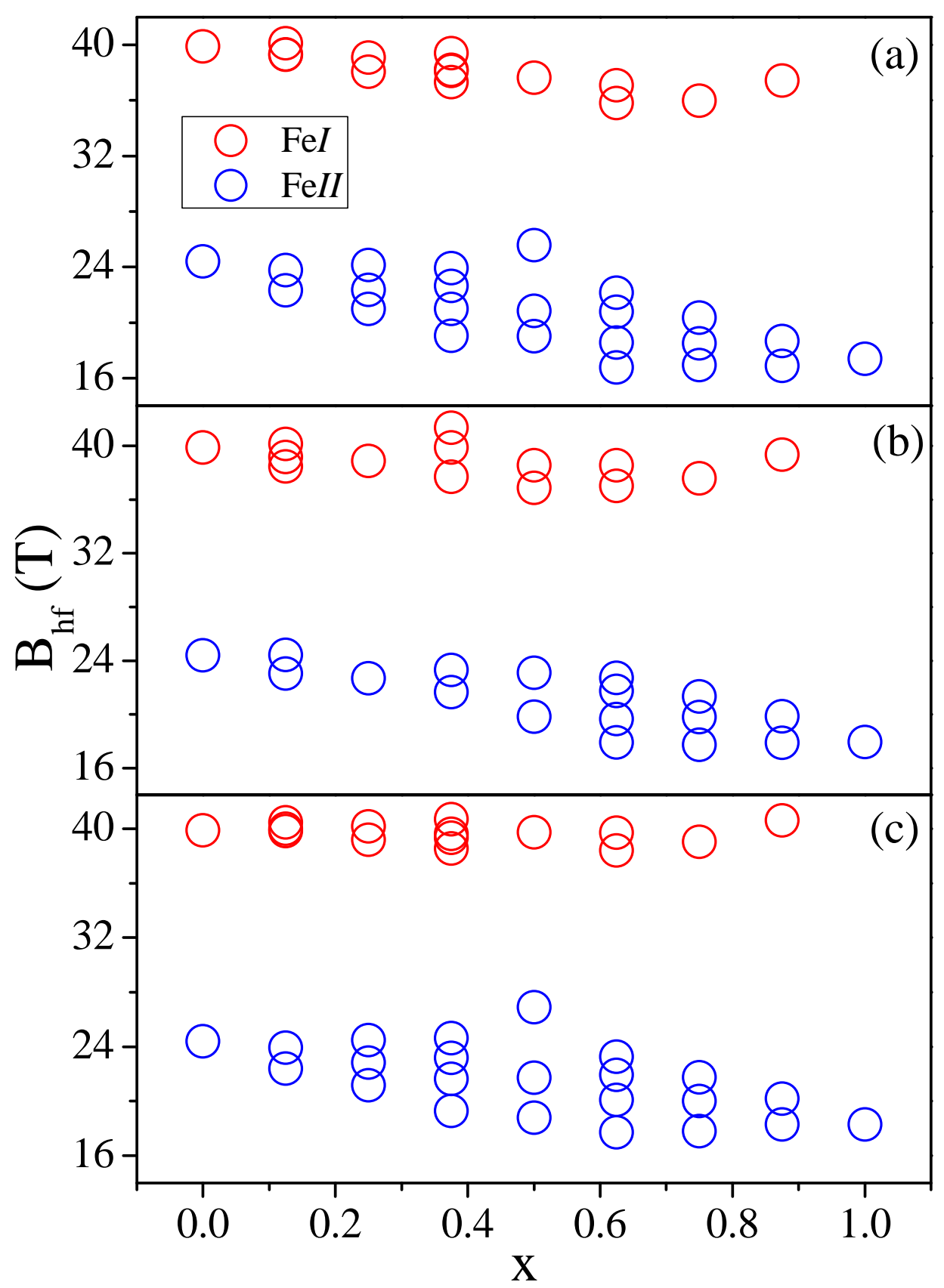

Figura 5.11: Dependencia del $B_{h f}$ con $x$ de los átomos FeI y FeII de los compuestos a) $\left.\mathrm{Al}_{x} \mathrm{Fe}_{4-x} \mathrm{~N}, \mathrm{~b}\right) \mathrm{Ga}_{x} \mathrm{Fe}_{4-x} \mathrm{~N}$ y c) $\operatorname{In}_{x} \mathrm{Fe}_{4-x} \mathrm{~N}$. 
La modificación del entorno de los átomos de Fe en los compuestos $M_{x} \mathrm{Fe}_{4-x} \mathrm{~N}$, no solo repercute en las propiedades magnéticas de dichos átomos, sino también en las propiedades hiperfinas de los mismos. En la Tabla 5.8 se reportan los valores del campo hiperfino $\left(B_{h f}\right)$, el corrimiento isomérico $(\delta)$ y el desdoblamiento cuadrupolar $(\varepsilon)$ de los distintos átomos de Fe de los compuestos $M_{x} \mathrm{Fe}_{4-x} \mathrm{~N}$. El cálculo de $B_{h f}, \delta$ y $\varepsilon$, fueron realizados considerando las expresiones (2.4), (2.5) y (2.6), respectivamente.

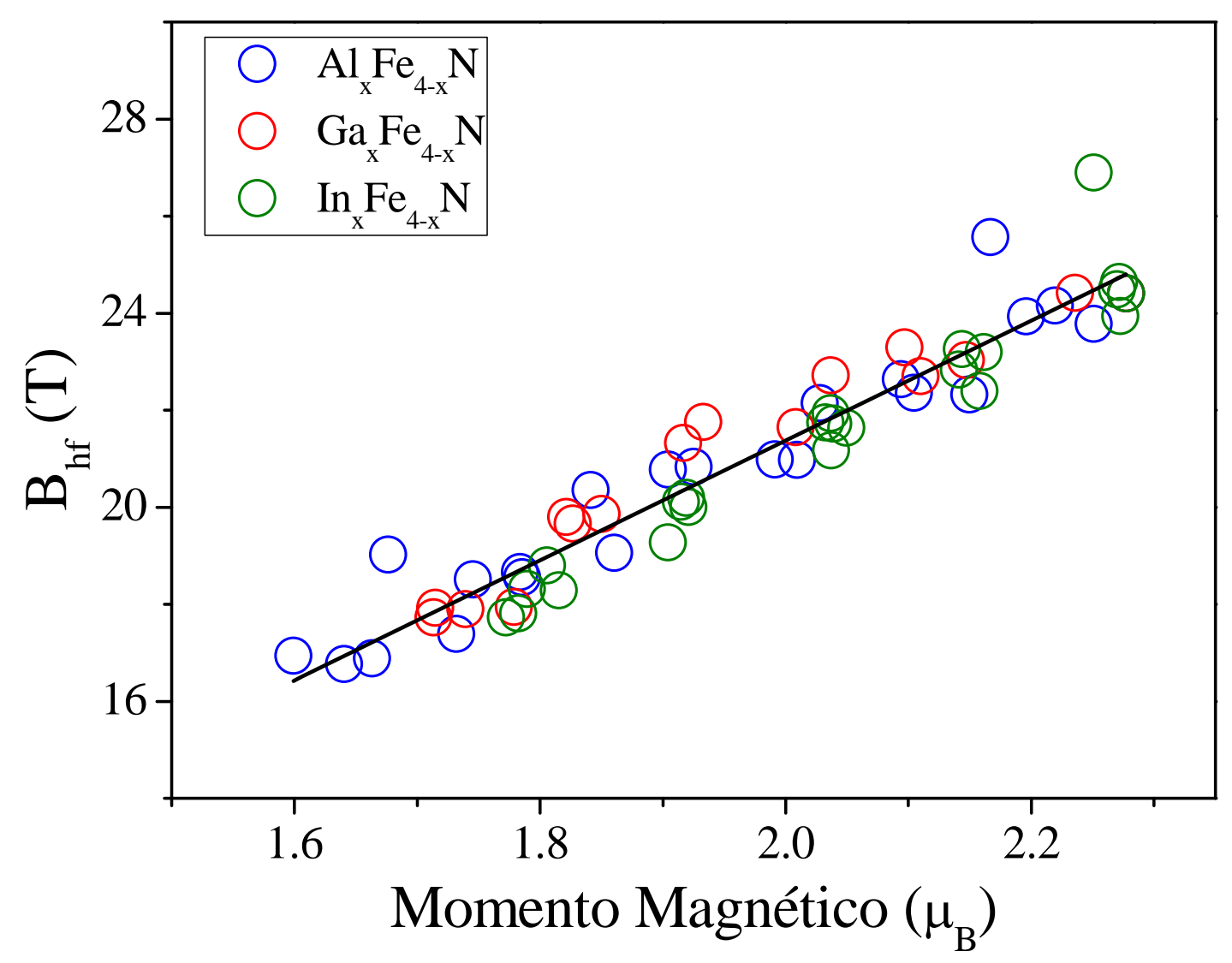

Figura 5.12: Dependencia del $B_{h f}$ con el momento magnético de los átomos FeII de los compuestos $M_{x} \mathrm{Fe}_{4-x} \mathrm{~N}$. 
En la Figura 5.11 se muestran los valores $B_{h f}$ de los átomos FeI y FeII, donde se observa un comportamiento semejante al momento magnético de los mismos, esto eso, el $B_{h f}$ de los FeI se mantiene casi constante con $x$, mientras que los $B_{h f}$ de los FeII disminuyen gradualmente con $x$. Esto se debe a que los electrones $3 d$-Fe polarizan a los electrones de los orbitales cercanos del núcleo, modificando así los correspondientes $B_{h f}$. La relación existente entre el momento magnético y el $B_{h f}$ de los átomos FeII puede ser cuantificada a través de la denominada constante de acoplamiento hiperfino $(A)$, en la Figura 5.12 se muestran los valores del momento magnético de los FeII versus su correspondiente $B_{h f}$ de los compuestos $\mathrm{Al}_{x} \mathrm{Fe}_{4-x} \mathrm{~N}$, $\mathrm{Ga}_{x} \mathrm{Fe}_{4-x} \mathrm{~N}$ y $\operatorname{In}_{x} \mathrm{Fe}_{4-x} \mathrm{~N}$, observándose una relación lineal entre ellas. Ajustando dichos datos con (2.8) se obtiene que $A=12.4 T / \mu_{B}$. Esto nos da una idea sobre la sensibilidad del $B_{h f}$ respecto a la redistribución de la carga en los orbitales $3 d$-Fe (Figura 5.7).

Complementariamente, en la Figura 5.13 se muestra el $B_{h f}$ de los FeII en función del número de átomos $M n n n$, observándose un decrecimiento lineal de $B_{h f}$ con pendientes de $-1.76 T,-1.65 T,-1.55 T$ por átomo $M$ nnn para los compuestos $\mathrm{Al}_{x} \mathrm{Fe}_{4-x} \mathrm{~N}, \mathrm{Ga}_{x} \mathrm{Fe}_{4-x} \mathrm{~N}$ y $\mathrm{In}_{x} \mathrm{Fe}_{4-x} \mathrm{~N}$, respectivamente, este tipo comportamiento del $B_{h f}$ ha sido mencionado en otras aleaciones de Fe-Ga $[198,199]$. 


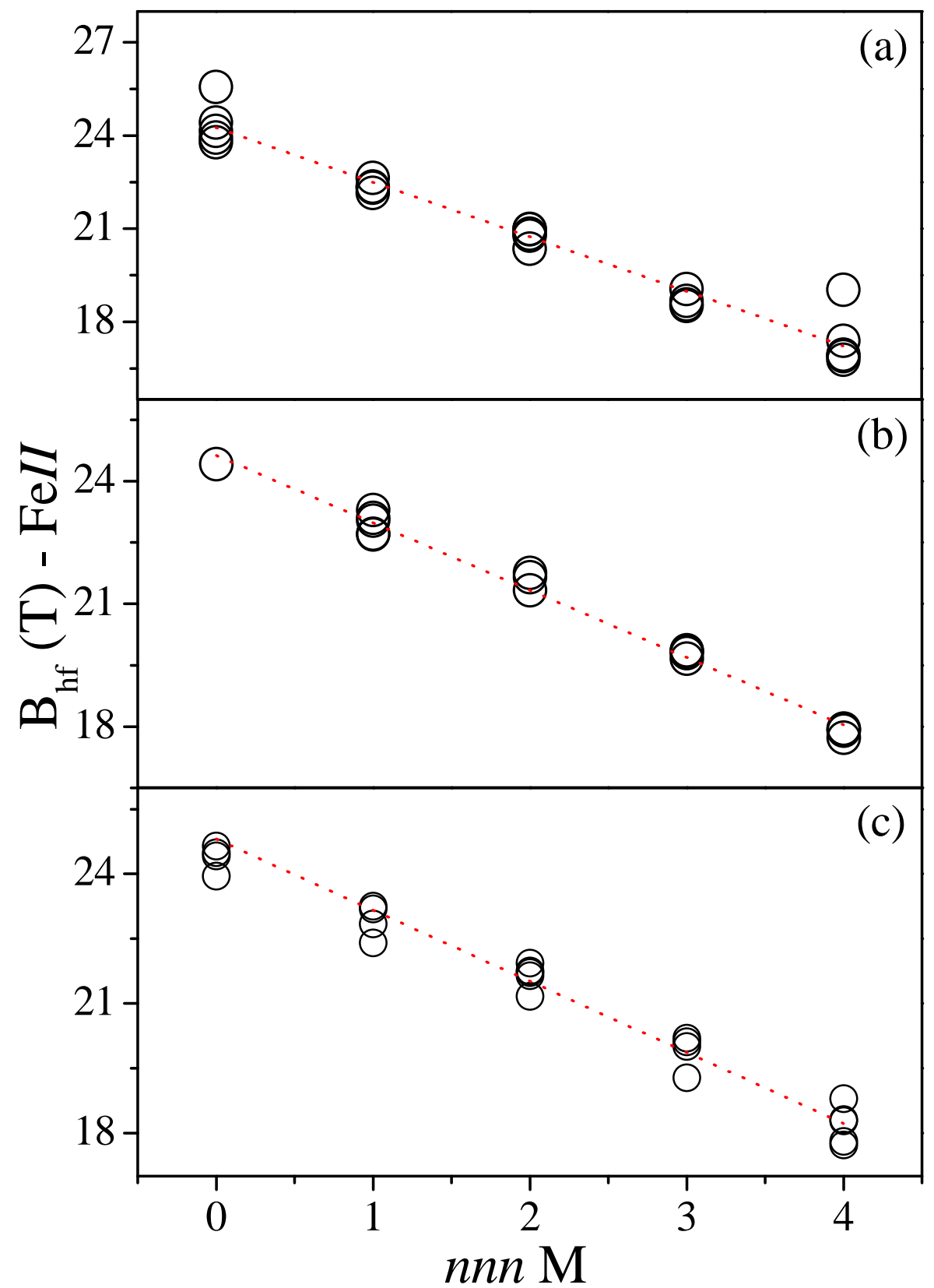

Figura 5.13: Dependencia del $B_{h f}$ de los FelI con el número de átomos $M$ nnn para los compuestos a) $\left.\mathrm{Al}_{x} \mathrm{Fe}_{4-x} \mathrm{~N}, \mathrm{~b}\right) \mathrm{Ga}_{x} \mathrm{Fe}_{4-x} \mathrm{~N}$ y c) $\mathrm{In}_{x} \mathrm{Fe}_{4-x} \mathrm{~N}$. 
Luego, los valores del $\delta$ y $\varepsilon$ para el caso de los FeI mantienen un valor casi constante respecto a $x$ (Tabla 5.8), lo cual indica que dichas propiedades hiperfinas no son afectadas por la sustitución de átomos de Fe por átomos $M$. Esto está relacionado con lo mencionado anteriormente, acerca que los FeI que siempre tendrán su mismo entorno, es decir, siempre tendrán la misma cantidad y el mismo tipo de átomos como $n n$ y $n n n$. Mientras que para los átomos FeII, el $\delta$ depende del número de átomos $M$ nnn. Para los compuestos $\mathrm{Al}_{x} \mathrm{Fe}_{4-x} \mathrm{~N}$ y $\mathrm{Ga}_{x} \mathrm{Fe}_{4-x} \mathrm{~N}$, se observa un incremento lineal de $\delta$ con el número de átomos M nnn (Figura 5.14a y 5.14b) con pendientes de $0.02 \mathrm{~mm} / \mathrm{s}$ y $0.04 \mathrm{~mm} / \mathrm{s}$ por átomo $M \mathrm{nnn}$, respectivamente; lo cual muestra una gradual disminución de la densidad de carga de los orbitales cercanos del núcleo de los s-FeII, conforme va aumentando la cantidad de átomos $M n n n$, un comportamiento semejante ha sido reportado en aleaciones solidas de Fe-Ga $[199,200]$. En los compuestos $\operatorname{In}_{x} \mathrm{Fe}_{4-x} \mathrm{~N}$ no se encontró el mismo comportamiento de $\delta$, donde tan solo se observa una mayor dispersion de los valores de $\delta$ respecto al número de átomos $M n n n$ (Figura 5.14c). Respecto del $\varepsilon$ de los FeII, en todos los compuestos se encontró un incremento lineal de este parámetro con referencia al número de átomos $M n n n$, con una pendiente de $0.10 \mathrm{~mm} / \mathrm{s}$ por átomo M nnn (Figura 5.15), representando un incremento de la asimetría esférica de la densidad de carga cercana al núcleo, donde el incremento del $\varepsilon$ representa indica una distribución de carga elipsoidal prolada. 


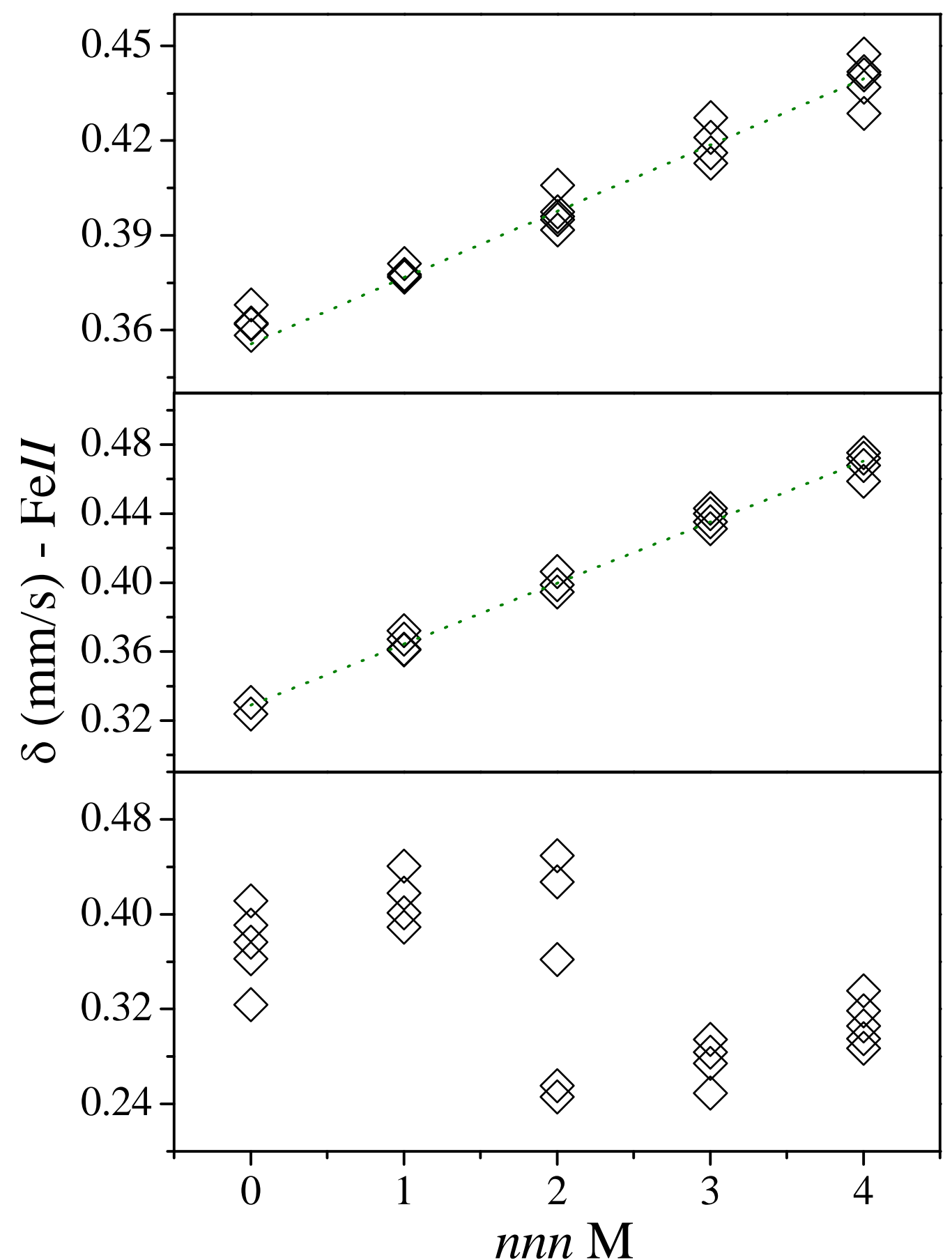

Figura 5.14: Variación del $\delta$ de los FeII en función del número de átomos $M$ nnn para los compuestos a) $\left.\mathrm{Al}_{x} \mathrm{Fe}_{4-x} \mathrm{~N}, \mathrm{~b}\right) \mathrm{Ga}_{x} \mathrm{Fe}_{4-x} \mathrm{~N}$ y c) $\operatorname{In}_{x} \mathrm{Fe}_{4-x} \mathrm{~N}$. 


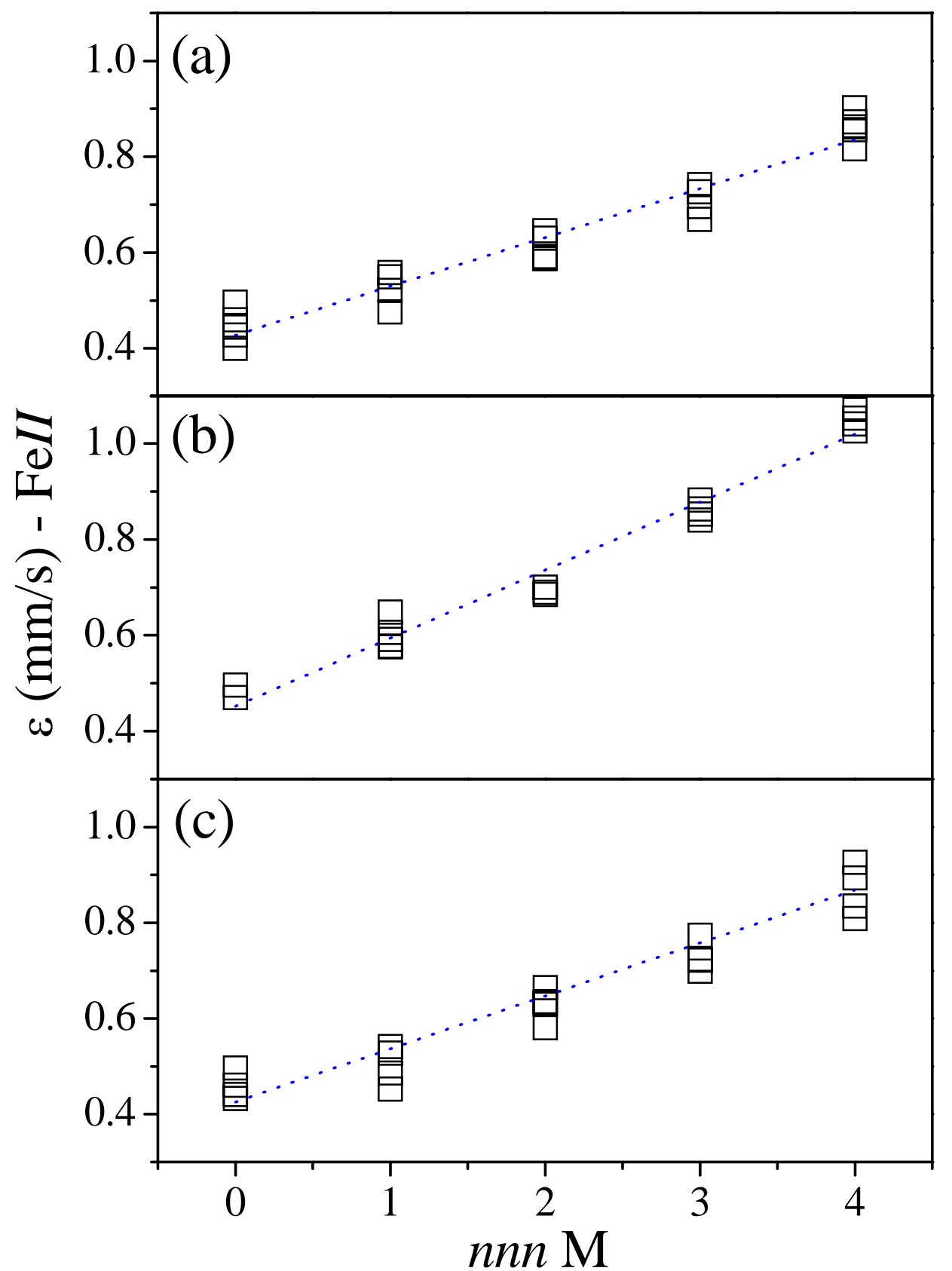

Figura 5.15: Variación del $\varepsilon$ de los FeII en función del número de átomos $M$ nnn para los compuestos a) $\left.\mathrm{Al}_{x} \mathrm{Fe}_{4-x} \mathrm{~N}, \mathrm{~b}\right) \mathrm{Ga}_{x} \mathrm{Fe}_{4-x} \mathrm{~N}$ y c) $\mathrm{In}_{x} \mathrm{Fe}_{4-x} \mathrm{~N}$. 


\subsection{Conclusiones}

Para los compuestos $M \mathrm{Fe}_{3} \mathrm{~N}$ se determinó que el átomo $M$ prefiere sustituir a un $\mathrm{Fe} I$ del $\gamma^{\prime}-\mathrm{Fe}_{4} \mathrm{~N}$, cuyo estado fundamental magnético es la configuración ferrimagnética, esto es, los momentos magnéticos de los átomos de Fe y $M$ están orientado en forma opuesta al del Fe produciendo así un $M_{f u}$ de casi la mitad del $\gamma^{\prime}-\mathrm{Fe}_{4} \mathrm{~N}$, mientras que el estado antiferromagnético se ubica en un mínimo local con una alta probabilidad de transición al estado ferrimagnético a través de una pequeña barrera de energía. Además se encontró una fuerte dependencia del parámetro de red (a) de dichos compuestos con el $M_{f u}$.

Consecuentemente, se pudo describir la dependencia de las propiedades estructurales, magnéticas e hiperfinas de los compuestos $M_{x} \mathrm{Fe}_{4-x} \mathrm{~N}$ en función de la concentración $(x)$. Donde se encontró una dependencia no lineal del parámetro de red $(a)$ con $x$, debido principalmente a una doble contribución proveniente de las diferencias de radios metálicos $\left(r_{\text {met }}\right)$ entre los átomos de Fe y $M$, y además al efecto magneto-volumétrico presente en estos compuestos. Para el caso del $\mathrm{Ga}_{x} \mathrm{Fe}_{4-x} \mathrm{~N}$ y $\operatorname{In}_{x} \mathrm{Fe}_{4-x} \mathrm{~N}$, la dependencia de $a$ con $x$, describe satisfactoriamente los valores experimentales obtenidos de la bibliografía.

El $M_{f u}$ del $M_{x} \mathrm{Fe}_{4-x} \mathrm{~N}$ decrece linealmente con $x$, lo cual está relacionado a que los átomos $M$ suprimen el momento magnético de los Fe por dilución. Donde la diferencia entre la densidades de estados up y down de los FeI no cambian, conllevando a una no dependencia con $x$ del momento magnético de los FeI. Para el caso de los FelI, el valor del momento magnético disminuye linealmente con el número de átomos $M n n n$, debido al tipo de enlace químico presente (parcialmente covalente), suprimiendo así el magnetismo de los Fell y además a la transferencia de estados electrónico $3 d$-up a $3 d$-down, pero manteniendo constante la carga total del orbital $3 d$. Las valores del $B_{h f}, \delta$ y $\varepsilon$ de los FeI se mantienen invariables respecto a $x$, mientras que para los átomos Fell se observa una dependencia con $x$. Se pudo establecer una relación lineal entre el momento magnético y el $B_{h f}$ de los átomos FeII determinándose así la constante de acoplamiento hiperfino $(A)$. 


\section{Bibliografía}

[1] K. Höck, H.J. Spies, B. Larish, G. Leonhardt, B. Buecken.

Surface and Coatings Technology 88 (1997) 44.

[2] H. Dong, Y. Sun, T. Bell.

Surface and Coatings Technology 90 (1997) 91.

[3] Y. Massiani, A. Medjahed, J.P. Crousier.

Surface and Coatings Technology 45 (1991) 115.

[4] M. Van Stappen, B. Malliet, L. Stals, L. De Schepper, J.R. Roos, J.P. Celis. Materials Science and Engineering A 140 (1991) 554.

[5] W.P. Tong, J. Sun, L. Zuo, J.C. He, J. Lu.

Wear 271 (2011) 653.

[6] J.M.D. Coey, P.A.I. Smith. Journal of Magnetism and Magnetic Materials 200 (1999) 405.

[7] I. Nakatani, M. Hijikata, K. Ozawa.

Journal of Magnetism and Magnetic Materials 122 (1993) 10.

[8] D.H. Mosca, P.H. Dionisio, W.H. Schreiner, J.R. Baumvol, C. Achete. Journal of Applied Physics 67 (1990) 7514.

[9] J.M.D. Coey.

Physica Scripta 21 (1991) T39.

[10] P.H. Emmett, S.B. Hendrick, S. Brunauer.

Journal of the American Chemical Society 52 (1930) 1456. 
[11] D. Andriamandroso, G. Demazeau, M. Pouchard, P. Hagenmüller. Journal of Solid State Chemistry 54 (1984) 54.

[12] B. Siberchicot, S.F. Matar, L. Fournès, G. Demazeau, P. Hagenmüller. Journal of Solid State Chemistry 84 (1990) 10.

[13] D. Andriamandroso, S. Matar, G. Demazeau, L. Fournès. IEEE Transactions on Magnetics 29 (1993) 2.

[14] S. Matar, L. Fournès, S. Chérubin-Jeannette, G. Demazeau. European Journal of Solid State and Inorganic Chemistry 30 (1993) 871.

[15] R.M. Martin, Electronic Structure, Basic Theory And Practical Methods.

Cambridge University Press, New York (2004).

[16] M. Born and R. Oppenheimer

Annalen der Physik 84 (1927) 457.

[17] D.R. Hartree.

Proceedings of the Cambridge Philosophical Society 24 (1928) 89.

[18] V.Z. Fock.

Zeitschrift für Physik 61 (1930) 209.

[19] A. Szabo, N.S. Ostlund, Modern QuAntum CHEMISTRY.

Dover Publishing, New York (1996).

[20] L.H. Thomas.

Proceedings of the Cambridge Philosophical Society 23 (1927) 542.

[21] E. Fermi.

Rend. Accad. Naz. Lincei 6 (1927) 602.

[22] P.A.M. Dirac.

Proceedings of the Cambridge Philosophical Society 26 (1930) 376.

[23] P. Hohenberg and W. Kohn.

Physical Review 136 (1964) B864. 
[24] W. Kohn and L.J. Sham. Physical Review 140 (1965) A1133.

[25] R.O. Jones and O. Gunnarsson. Reviews of Modern Physics 61 (1989) 689.

[26] D.M. Ceperley and B.J. Alder. Physical Review Letters 45 (1980) 566.

[27] S.H. Vosko, L. Wilk and M. Nusair. Canadian Journal of Physics 58 (1980) 1200.

[28] J.P. Perdew and A. Zunger. Physical Review B 23 (1981) 5048.

[29] L.A. Cole and J.P. Perdew. Physical Review A 25 (1982) 1265.

[30] J.P. Perdew and Yue Wang. Physical Review B 45 (1992) 13244.

[31] J.P. Perdew and K. Burke. International Journal of Quantum Chemistry 57 (1996) 309.

[32] A.D. Becke. Physical Review A 38 (1988) 3098.

[33] J.P. Perdew, J.A. Chevary, S.H. Vosko, K.A. Jackson, M.R. Pederson and C. Fiolhais.

Physical Review B 46 (1992) 6671.

[34] J.P. Perdew, K. Burke and M. Ernzerhof.

Physical Review Letters 77 (1996) 3865.

[35] Y. Zhang and W. Yang.

Physical Review Letters 80 (1998) 890.

[36] H. Hammer and Norskov. Physical Review B 59 (1999) 7413. 
[37] Z. Wu and R.E. Cohen.

Physical Review 73 (2006) 235116.

[38] J.P. Perdew, A. Ruzsinszky, G.I. Csonka, O.A. Vydrov, G.E. Scuseria, L.A. Constantin, X. Zhou, and K. Burke.

Physical Review Letters 100 (2008) 136406.

[39] J.M. MacLaren, D.P. Clougherty, R.C. Albers.

Physical Review B 42 (1990) 3205.

[40] D.J. Singh, W.E. Pickett, H. Krakauer.

Physical Review B 43 (1991) 11628.

[41] D.J. Singh, D.P. Clougherty, J.M. MacLaren, R.C. Albers, C.S. Wang. Physical Review B 44 (1991) 7701.

[42] S. Cottenier, Density Functional Theory AND the FAMily of (L)APWMETHODS: A STEP-BY-STEP INTRODUCTION.

Instituut voor Kern-en Stralingsfysica, K.U. Leuven, Belgium, ISBM 90807215-1-4 (2002).

[43] J.C. Slater.

Physical Review 51 (1937) 846.

[44] T.L. Loucks, Augmented Plane WaVe Method: A Guide TO PERFORMING ELECTRONIC STRUCTURE CALCULATIONS.

Benjamin Edt., New York (1967).

[45] O.K. Andersen.

Physical Review B 12 (1975) 3060.

[46] D.J. Singh, Planewaves, Pseudopotentials, and the APW Method. Kluwer Academic Publisher, Boston (1994).

[47] H. Hägg.

Zeitschrift für Physikalische Chemie B 8 (1930) 455.

[48] K.H. Jack.

Proceedings of the Royal Society A 195 (1948) 34. 
[49] H. Jacobs, J. Bock.

Journal of the Less-Common Metals 134 (1987) 215-220.

[50] H. Jacobs, D. Rechenbach, U. Zachwieja.

Journal of Alloys and Compounds 227 (1995) 10.

[51] N. W. Ashcroft, N.D. Mermin, SOlid State Physics.

Holt-Rinehart-Winston, New York (1976).

[52] B.C. Frazer.

Physical Review 112 (1958) 751.

[53] G. Shirane, W.J. Takei, S.L. Ruby.

Physical Review 126 (1962) 49.

[54] C.A. Kuhnen, R.S. de Figueiredo, V. Drago, E.Z. da Silva.

Journal of Magnetism and Magnetic Materials 111 (1992) 95.

[55] Q. Qi, K. O’Donnel, E. Touchais J.M.D. Coey.

Hyperfine Interactions 94 (1994) 2067.

[56] T. Yamaguchi, M. Sakita, M. Nakamura, T. Kobira.

Journal of Magnetism and Magnetic Materials 215 (2000) 529.

[57] S.K. Chen, S. Jin, T.H. Tiefel, Y.F. Hsieh, E.M. Gyorgy, D.W. Johnson Jr. Journal of Applied Physics 70 (1991) 6247.

[58] J.C. Wood, A.J. Nozik.

Physical Review 4 (1971) 2224.

[59] J.L. Costa-Krämer, D. M. Borsa, J.M. García-Martín, M.S. Martín-González, D.O. Boerma, F. Briones.

Physical Review B 69 (2004) 144402.

[60] O. Bartels, K.D. Becker.

Zeitschrift für Physikalische Chemie 221 (2007) 1509.

[61] F. Mahboubi, M. Samandi, D. Dunne, A. Bloyce, T. Bell. Surface and Coatings Technology 71 (1995) 135. 
[62] M. Berg, C.V. Budtz-Jørgensen, H. Reitz, K.O. Schweitz, J. Chevallier, P. Kringhøj.

Surface and Coatings Technology 124 (2000) 25.

[63] J.F. Adler, Q. Williams.

Journal of Geophysical Research B 110 (2005) 1203.

[64] M.F. Yan, Y.Q. Wu, R.L. Liu.

Applied Surface Science 255 (2009) 8902.

[65] Z.Q. Liu, H. Hashimoto, M. Song, K. Mitsuishi, K. Furuya.

Acta Materialia 52 (2004) 1669.

[66] R.N. Panda and N.S. Gajbhiye.

IEEE Transactions on Magnetics 34 (1998) 542.

[67] F. Tessier, A. Navrotsky, R. Niewa, A. Leineweber, H. Jacobs, S. Kikkawa, M.

Takahashi, F. Kanamaru, F.J. DiSalvo.

Solid State Science 2 (2000) 457.

[68] N.S. Gajbhiye, R.N. Panda, R.S. Ninghthoujam, S. Bhattacharyya.

Physica Status Solidi c 1 (2004) 3252.

[69] M. Cao, T. Liu, G. Sun, X. Wu, X. He, C. Hu.

Journal Solid State Chemistry 178 (2005) 2390.

[70] P. Schaaf.

Hyperfine Interactions 111 (1998) 113.

[71] T. Takahashi, N. Takahashi, N. Tamura, T. Nakamura, M. Yoshioka, W. Inami, Y. Kawata.

Journal of Materials Chemistry 11 (2001) 3154.

[72] S. Yu Grachev, D.M. Borsa, D.O. Boerma.

Surface Science 515 (2002) 359.

[73] X. Wang, W.T. Zheng, H.W. Tian, S.S. Yu, L.L. Wang.

Journal of Magnetism and Magnetic Materials 283 (2004) 282. 
[74] C. Navio, J. Alvarez, M.J. Capitan, F. Ynduráin, R. Miranda. Physical Review B 78 (2008) 155417.

[75] A. Narahara, K. Ito, T. Suemasu. Journal of Crystal Growth 311 (2009) 1616.

[76] Y. Takagi, K. Isami, I. Yamamoto, T. Nakagawa, T. Yokoyama. Physical Review B 81 (2010) 035422.

[77] K. Ito, G. H. Lee, H. Akinaga, T. Suemasu. Journal of Crystal Growth 322 (2011) 63.

[78] K. Ito, G.H. Lee, K. Harada, M. Suzuno, T. Suemasu, Y. Takeda, Y. Saitoh, M. Ye, A. Kimura and H. Akinaga. Applied Physics Letters 98 (2011) 102507.

[79] D.M. Borsa, D.O. Boerma. Hyperfine Interactions 151 (2003) 31.

[80] X. Wang, W.T. Zheng, H.W. Tian, S.S. Yu, W. Xu, S.H. Meng, X.D. He, J.C. Han, C.O. Sun, B.K. Tay. Applied Surface Science 220 (2003) 30.

[81] J.M. Gallego, S. Yu. Grachev, D.M. Borsa, D.O. Boerma, D. Écija, R. Miranda. Physical Review B 70 (2004) 115417.

[82] J.M. Gallego, D.O. Boerma, R. Miranda, F. Ynduráin. Physical Review Letters 95 (2005) 136102.

[83] H. Xiang, F.Y. Shi, M.S. Rzchowski, P.M. Voyles, Y.A. Chang. Journal of Applied Physics 109 (2011) 07E126.

[84] L. Zhang, T. Ma, Z. Ahmad, T. Yuan, C. Lu, Y. Xu, M. Yan. Journal of Alloys and Compounds 509 (2011) 5075.

[85] W.B. Mi, F. He, Z.Q. Li, P. Wu, E.Y. Jiang, H.L. Bai. Journal of Physics D: Applied Physics 39 (2006) 911.

[86] Y.D. Zhang, J.I. Budnick, W.A. Hines, M.Q. Huang and W.E. Wallace. Physical Review B 54(1) (1996) 51. 
[87] T. Takahashi, N. Takahashi, T. Nakamura, T, Kato, K. Furukawa, G.M. Smith, P.C. Riedi.

Solid State Science 6 (2004) 97.

[88] A. Pösinger, J. Bogner, W. Steiner, P. Mohn, K. Schwarz, S. Matar, G. Demazeau. Hyperfine Interactions 94 (1994) 2093.

[89] J.S. Lord, J.G.M. Armitage, P.C. Riedi, S.F. Matar, G. Demazeau. Journal of Physics: Condensed Matter 6 (1994) 1779.

[90] C.L. Yang, M.M. Abd-Elmeguid, H. Micklitz, G. Michels, J.W. Otto, Y. Kong, D.S. Xue and F.S. Li. Journal of Magnetism and Magnetic Materials 151 (1995) L19.

[91] P. Rochegude, J. Foct.

Physica Status Solidi a 98 (1986) 51.

[92] J.Q. Xiao and C.L. Chien.

Applied Physics Letters 64 (1994) 384.

[93] Y. Koltypin, X. Cao, R. Prozorov, J. Balogh, D. Kaptas, A. Gedanken. Journal of Materials Chemistry 7 (1997) 2453.

[94] L.L. Wang, W.T. Zheng, J. Gong, H.B. Li, X. Wang, N. Ma, P.J. Cao, X.C. Ma. Journal of Alloys and Compounds 467 (2009) 1.

[95] A.J. Nozik, J.C. Wood, Jr. And G. Haacke. Solid State Communications 7 (1969) 1677.

[96] R.S. Figueiredo, V. Drago. Solid State Communications 80 (1991) 757.

[97] Kyu-Jin Kim, K. Sumiyama, K. Shibata, K. Suzuki. Materials Science and Engineering A 181 (1994) 1272.

[98] P. Schaaf, C. Illgner, M. Niederdrenk, K.P. Lieb. Hyperfine Interactions 95 (1995) 199.

[99] F.A.O. Cabral, J.H. de Araujo, R.C. Araujo, S. Gama. Journal of Applied Physics 83 (1998) 6932. 
[100] R.N. Panda, B. Balaji, N.S. Gajbhiye. Hyperfine Interactions 141 (2002) 187.

[101] L.L. Wang, X. Wang, W.T. Zheng, N. Ma, Q.F. Guan, J. Zhao, Y. Chen, S.H. Feng.

Materials Chemistry and Physics 100 (2006) 304.

[102] J.F. Marco, T. Herranz, M. Garcia, J.R. Gancedo, F. Moutinho, P. Prieto and J.M. Sanz.

Surface and Interface Analysis 42 (2010) 616.

[103] R.J. Arnott and A. Wold. Journal of Physics and Chemistry of Solids 15 (1960) 152.

[104] D. Xue, F. Li, J. Yang, Y. Kong, M. Gao. Journal of Magnetism and Magnetic Materials 172 (1997) 165.

[105] X.G. Diao, A.Y. Takeuchi, F. Garcia, R.B. Scorzelli, H. R. Rechenberg. Journal of Applied Physics 85 (1999) 8.

[106] R.N. Panda and N.S. Gajbhiye. Journal of Magnetism and Magnetic Materials 195 (1999) 396.

[107] F.S. Li, Z.J. Zhao, X.G. Diao, D.S. Xue. Physica Status Solidi a 174 (1999) 255.

[108] D. Music, J. M. Scheider. Applied Physics Letters 88-3 (2006) 031914.

[109] S. El Khiraoui, M. Sajieddine, M. Vergat, M Sahlaoui, Ph. Bauer, M. Mabrouki. Journal of Alloys and Compounds 440 (2007) 43.

[110] L.L. Wang, W.T. Zheng, T. An, N. Ma, J. Gong. Journal of Alloys and Compounds 495 (2010) 265.

[111] R.S. Figueiredo, F. Foct, A.V. dos Santos, C.A. Kuhnen. Journal of Alloys and Compounds 315 (2001) 42.

[112] S. El Khiraoui, M. Sajieddine, M. Vergat, Ph. Bauer, M. Mabrouki. Physica B 388 (2007) 180. 
[113] C.A. Kuhnen, R.S. de Figueiredo, A.V. dos Santos. Journal of Magnetism and Magnetic Materials 219 (2000) 58.

[114] T. Takahashi, D. Music, J. M. Schneider. Scripta Materialia 65 (2011) 380.

[115] A. Houben, V. Sepelák, K.D. Becker, R. Dronskowski. Chemistry of Materials 21 (2009) 784.

[116] A. Houben, P. Müller, J. von Appen, H. Lueken, R. Niewa, R. Dronskowski. Angewandte Chemie 44 (2005) 7212.

[117] D. Music, J. Burghaus, T. Takahashi, R. Dronskowski, J.M. Schneider. The European Physical Journal B 77 (2010) 401.

[118] T. Takahashi, D. Music, J. M. Schneider. Journal of Vacuum Science E Technology A 30-3 (2012) 030602-1.

[119] S.K. Chen, S. Jin, G.W. Kammlott, T.H. Tiefel, D.W. Johnson Jr., E.M. Gyorgy. Journal of Magnetism and Magnetic Materials 110 (1992) 65.

[120] Y. Kong, F. Li.

Physical Review B 57 (1998) 2.

[121] R.N. Panda, N.S. Gajbhiye. Journal of Applied Physics 86 (1999) 6.

[122] N.S. Gajbhiye, S. Bhattacharyya. Indian Journal of Pure and Applied Physics 45 (2007) 834.

[123] A.V. dos Santos, C.A. Kuhnen. Journal of Alloys and Compounds 321 (2001) 60.

[124] A.V. dos Santos, J.C. Krause, C.A. Kuhnen. Physica B 382 (2006) 290.

[125] C.A. Kuhnen, A.V. dos Santos. Journal of Magnetism and Magnetic Materials 130 (1994) 353.

[126] L. Chen. Journal of Applied Physics 100 (2006) 113717. 
[127] C.A. Kuhnen, A.V. dos Santos.

Journal of Alloys and Compounds 297 (2000) 68.

[128] C. Paduani.

Journal of Applied Physics 96-3 (2004) 1503.

[129] E.L. Peltzer y Blancá, J. Desimoni, N.E. Christensen, H. Emmerich, S. Cottenier.

Physica Status Solidi b 246 (2009) 909.

[130] A.V. Gil Rebaza, J. Desimoni, E.L. Peltzer y Blancá.

Physica B 404 (2009) 2872,

[131] S. Shang and A.J. Böttger.

Acta Materialia 53 (2005) 255.

[132] T. Gressmann, M. Wohlschlögel, S. Shang, U. Welzel, A. Leineweber, E.J. Mittemeijer, Z.K. Liu.

Acta Materialia 55 (2007) 5833.

[133] R. Niewa, D. Rau, A. Wosylus, K. Meier, M. Wessel, M. Hanfland, R. Dronskowski, U. Schwarz.

Journal of Alloys and Compounds 480 (2009) 76.

[134] T. Takahashi, J. Burghaus, D. Music, R. Dronskowski, J.M. Schneider. Acta Materialia 60 (2012) 2054.

[135] Z. Wu, J. Meng.

Applied Physics Letters 90 (2007) 241901.

[136] X.G. Ma, J.J. Jiang, P. Liang, J. Wang, Q. Ma, Q.K. Zhang. Journal of Alloys and Compounds 480 (2009) 475.

[137] J. von Appen, R. Dronskowski.

Angewandte Chemie 44 (2005) 1205.

[138] P. Mohn, K. Schwarz, S. Matar, G. Demazeau.

Physical Review B 45-8 (1992) 4000. 
[139] Y.Q. Wu, M.F. Yan.

Physica B 405 (2010) 2700.

[140] L. Chen.

Journal of Magnetism and Magnetic Materials 303 (2006) 266.

[141] S. Matar, P. Mohn, G. Demazeu, K. Schwarz.

Journal of Magnetism and Magnetic Materials 101 (1991) 251.

[142] C. Paduani.

Journal of Magnetism and Magnetic Materials 278 (2004) 231.

[143] E. Zhao, H. Xiang, J. Meng, Z. Wu.

Chemical Physics Letters 449 (2007) 96.

[144] S. Matar, A. Houari, M. A. Belkhir, M. Zakhour.

Zeitschrift für Naturforschung 62b (2007) 881.

[145] R.S. de Figueiredo, C.A. Kuhnen, A.V. dos Santos.

Journal of Magnetism and Magnetic Materials 173 (1997) 141.

[146] F. Li, J. Yang, D. Xue, R. Zhou.

Applied Physics Letters 66 (1995) 2343.

[147] H.R. Rechenberg, X.G. Diao, R.B. Scorzelli.

Journal of Applied Physics 87 (2000) 9.

[148] T. Sanai, K. Ito, K. Toko, T. Suemasu.

Journal of Crystal Growth 357 (2012) 53.

[149] J. Burghaus, M. Wessel, A. Houben, R. Dronskowski.

Inorganic Chemistry 49 (2010) 10148.

[150] J. Burghaus, M. T. Sougrati, A. Möchel, A. Houben, R. P. Hermann, R. Dronskowski.

Journal of Solid State Chemistry 184 (2011) 2315.

[151] S. Kurian, S. Bhattacharyya, J. Desimoni, E.L. Peltzer y Blancá, A. V. Gil Rebaza, N.S. Gajbhiye.

The Journal of Physical Chemistry C 114 (2010) 17542. 
[152] J. Burghaus, J. Kleemann, R. Dronskowski.

Zeitschrift für Anorganische und Allgemeine Chemie 637 (2011) 1.

[153] D. Adriamandroso, L. Fefilatiev, G. Demazeau, L. Fournès, M. Pouchard. Materials Research Bulletin 19 (1984) 1187.

[154] Z.J. Zhao, D.S. Xue, F.S. Li. Journal of Magnetism and Magnetic Materials 232 (2001) 155.

[155] Houben, J. Burghaus, R. Dronskowski.

Chemistry of Materials 21 (2009) 4332.

[156] A.V. Gil Rebaza, J. Desimoni, S. Kurian, S. Bhattacharyya, N. S. Gajbhiye, E.L. Peltzer y Blancá.

The Journal of Physical Chemistry C 115 (2011) 23081.

[157] P. Blaha, K. Schwarz, G. Madsen, D. Kvasnicka, and J. Luitz. WIEN2k, An Augmented Plane Wave + Local Orbitals Program for Calculating Crystal Properties (Karlheinz Schwarz, Tech. Universität Wien, Austria, 1999), ISBN 3-9501031-1-2. (http:/ /www.wien2k.at).

[158] F.D. Murnaghan.

Proceedings of the National Academy of Sciences of the USA 30 (1944) 244.

[159] F. Birch.

Physical Review 77 (1947) 809.

[160] P. Götlich, E. Bill and A.X. Trautwein, MÖssbauer Spectroscopy AND Transition Metal Chemistry, Fundamentals and Applications. Springer Edt., Berlin (2011).

[161] A. Vértes, L. Korecz and K. Murger, Mössbauer Spectroscopy. Elsevier - North-Holland Inc., Amsterdam (1979).

[162] S.M. Dubiel. Journal of Alloys and Compounds 488 (2009) 18.

[163] S. Blügel, H. Akai, R. Zeller, P.H. Dederichs. Physical Review B 35 (1987) 3271. 
[164] O. Eriksson, A. Svane. Journal of Physics: Condensed Matter 1 (1989) 1589.

[165] P. Dufech, P. Blaha, K. Schwarz. Physical Review Letters 75 (1995) 3545.

[166] R.E. Watson, A.J. Freeman. Physical Review 123 (1961) 2027.

[167] I. Vincze, I.A. Campbell, A.J. Meyer. Solid State Communications 15 (1974) 1495.

[168] P. Novák, V. Chlan. Physical Review B 81 (2010) 174412.

[169] H. Akai, M. Maki, S. Blügel, R. Zeller, P.H. Dederichs. Journal of Magnetism and Magnetic Materials 45 (1984) 291.

[170] M.E. Elzain, D.E. Ellis, D. Guenzburger. Physical Review B 34 (1986) 1430.

[171] S.N. Mishra, S.K. Srivastava. Journal of Physics: Condensed Matter 20 (2008) 285204.

[172] V. Niculescu, J.I. Budnick, W.A. Hines, K. Raj, S. Pickart, S. Skalski. Physical Review B 19 (1979) 452.

[173] J. Cieslak, M. Reissner, S.M. Dubeil, W. Steiner. Physica Status Solidi a 205 (2008) 1791.

[174] A.V. Gil Rebaza, J. Desimoni, E.L. Peltzer y Blancá. Physica B 407 (2012) 3240.

[175] J. Friedel. Nuovo Cimento Supplement 2 (1958) 287.

[176] S. F. Matar. Journal of Alloys and Compounds 345 (2002) 72.

[177] A. Houari, S.F. Matar, M. A. Belkhir. Journal of Magnetism and Magnetic Materials 322 (2010) 658. 
[178] A. Houari, S.F. Matar, M. A. Belkhir. Journal of Magnetism and Magnetic Materials 312 (2007) 298.

[179] S. Baroni, A.D. Corso, S. de Gironcoli, P. Giannozzi, C. Cavazzoni, G. Ballabio, S. Scandolo, G. Chiarotti, P. Focher, A. Pasquarello, K. Laasonen, A. Trave, R. Car, N. Marzari, A. Kokalj, www.quantum-espresso.org

[180] J. M. D. Coey, Magnetism and Magnetic Materials.

Cambridge University Press, New York (2010).

[181] S. Matar, P. Mohn and J. Kübler. Journal of Magnetism and Magnetic Materials 104 ( 1992) 1927.

[182] D.C. Khan, M. Mishra, A.R. Das.

Journal of Applied Physics 53 (1982) 2722.

[183] S.T. Alone, K.M. Jadhav.

Pramana - Journal of Physics 70 (2008) 173.

[184] K.E. Sickafus, J.M. Wills, N.W. Grimes. Journal of the American Ceramic Society 82 (1999) 3279.

[185] A.A. Pandit, S.S. More, R.G. Dorik, K.M. Jadhav. Bulletin of Material Science 26 (2003) 517.

[186] K. Muraleedharan, J.K. Srivastava, V.R. Marathe, R. Vijayaraghavan. Journal of Physics C: Solid State Physics 18 (1985) 5897.

[187] P.B. Pandya, H.H. Joshi, R.G. Kulkarni. Journal of Materials Science 26 (1991) 5509.

[188] U. V. Chhaya, B.S. Trivedi, R.G. Kulkarni. Physica B 262 (1999) 5.

[189] H.M. Zaki. Physica B 407 (2012) 2025.

[190] L. Pauling. Journal of the American Chemical Society 69 (1947) 542. 
[191] A. Houben, J. Burghaus, R. Dronskowski.

Chemistry of Materials 23 (2011) 3580.

[192] C.A. Kuhnen, A.V. dos Santos.

Journal of Alloys and Compounds 384 (2004) 80.

[193] A. Kellou, J.M. Raulot, T. Grosdidier.

Intermetallic 18 (2010) 1293.

[194] Y. Ouyang, M. Tang, J. Fang, P. Xiang, Y. Du, Y. He, Y. Liu.

Physica Scripta 80 (2009) 055603.

[195] A. Kellou, T. Grosdidier, J.M. Raulot, H. Aourag.

Physica Status Solidi b 245 (2008) 750.

[196] W. Zhang.

Journal of Magnetism and Magnetic Materials 323 (2011) 2206.

[197] K.T. Jacob, S. Raj, L. Rannesh.

International Journal of Materials Research 9 (2007) 776.

[198] A.T. Aldred.

Journal of Applied Physics 37 (1966) 1344.

[199] A. Blachowski, K. Ruebenbauer, J. Zukrowski, J. Przewoznik. Journal of Alloys and Compounds 455 (2008) 47.

[200] L.R. Newkirk, C.C. Tsuei.

Physical Review B 4 (1971) 4046. 